DANMARKS GEOLOGISKE UNDERS $\varnothing$ GELSE · SERIE A · NR. 6 MILJØMINISTERIET · Geological Survey of Denmark

\title{
Catalogue of late- and post-glacial macrofossils of Spermatophyta from Denmark, Schleswig, Scania, Halland, and Blekinge dated 13,000 B.P. to 1536 A.D.
}

BY

HANS ARNE JENSEN

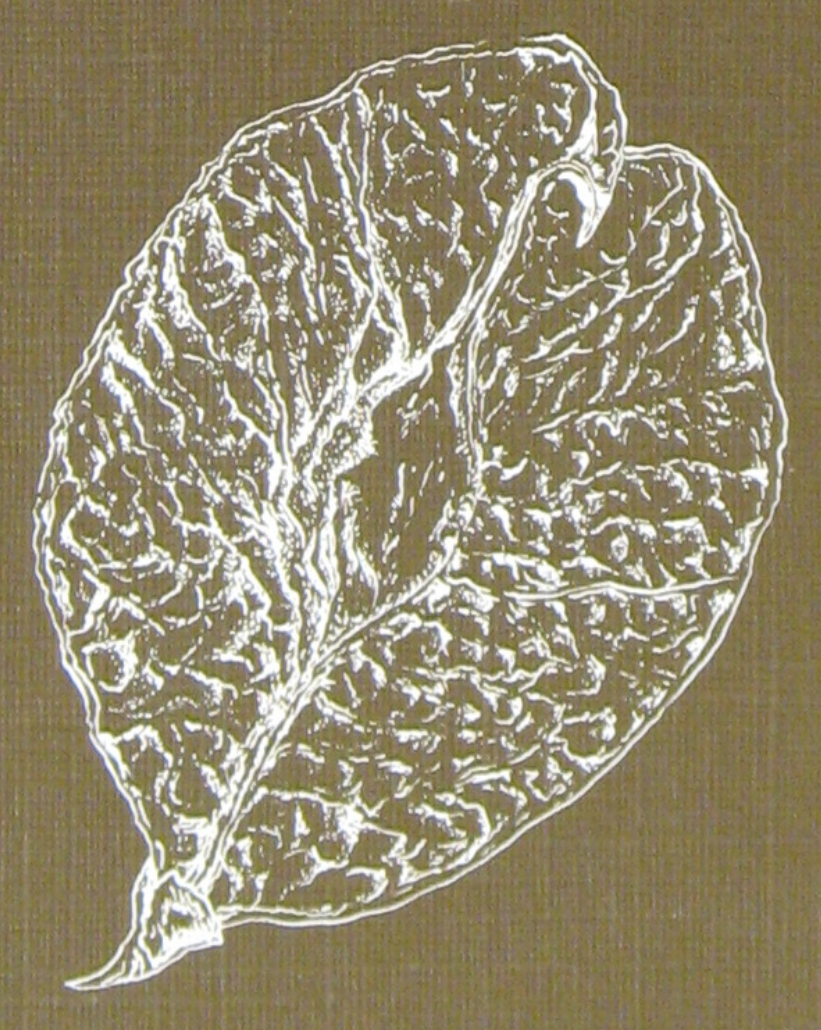

I kommission hos C.A. Reitzels forlag · København 1985 


\section{Catalogue of late- and post-glacial macrofossils of Spermatophyta from Denmark, Schleswig, Scania, Halland, and Blekinge dated 13,000 B.P. to 1536 A.D.}

BY

HANS ARNE JENSEN

I kommission hos C.A. Reitzels forlag - København 1985 
Front cover: Ulmus glabra, Drawing by Elisabeth Papp.

DGU SERIE A nr. 6

ISBN 87-421-0737-7

ISSN 0901-0270

Oplag: 1200

Sats, montage og tryk: AiO Tryk as, Odense

Lay-out, tilretteloggelse: Walter Rayher

Dato: 1985-12-20

Hans Arne Jensen. Danish State Seed Testing Station

Skovbrynet 20. DK-2800 Lyngby

Redaktion: Bent Aaby

(C) Danmarks Geologiske Undersøgelse

Thoravej 31, DK-2400 København NV 


\section{Contents}

Abstract $\ldots \ldots \ldots \ldots \ldots \ldots \ldots \ldots \ldots \ldots \ldots \ldots$

Introduction $. . \ldots \ldots \ldots \ldots \ldots \ldots \ldots \ldots \ldots, 7$

Previous macrofossil surveys ................ 9

The catalogue ........................ 10

Concluding remarks................... 16
Acknowledgments..................... 17

References .......................... 18

Table $1 \ldots \ldots \ldots \ldots \ldots \ldots \ldots \ldots \ldots \ldots, \quad 24$

Table $2 \ldots \ldots \ldots \ldots \ldots \ldots \ldots \ldots \ldots \ldots \ldots, \quad 36$

Table $3 \ldots \ldots \ldots \ldots \ldots \ldots \ldots \ldots \ldots \ldots . \quad 79$ 


\section{Abstract}

The catalogue summarizes published finds of macrofossils from 551 taxa of Spermatophyta originating from 505 sites in Denmark, Schleswig, Scania, Halland, and Blekinge and dated to periods between 13,000 B.P. and 1536 A.D. The information is arranged in one map and three tables. The map shows the position of each find. Table 1 presents the sites by number and name, where the finds are published, their age, the dating method applied, and the media examined. Table 2 lists the finds of macrofossils in pollen assemblage zones I-IX and the periods Pre-Roman Iron Age, Roman Iron Age, Germanic Iron Age, Viking Age, Early Middle Ages, and Late Middle Ages. Table 3 summarizes by family the finds of macrofossils in these periods. 


\section{Introduction}

Palaeobotanical studies have revealed that the flora of Denmark and adjacent areas has changed extensively since the ice withdrew about 13,000 B.P. (C-14 years before 1950 A.D.).

During my work on macrofossils from archaeologically dated soil samples (Jensen 1979a, 1979b) and in subsequent studies, I have found a strong need for a survey of the available information on where and when the recognized species have been found.

A comprehensive Danish bibliography covering the subject does not exist, and an attempt has therefore been made to summarize the relevant literature dealing with finds of macrofossils of higher plants (Spermatophyta) from Denmark, Schleswig, Scania, Halland, and Blekinge. The bibliography covers the period from the Last Ice Age (Late Weichselian - about 13,000 B.P.) to the end of the Middle Ages (1536 A.D.).

The work has been impeded by the fact that the information is scattered in various botanical, zoological, and archaeological journals and books; and the titles do not always disclose whether the paper contains information on macrofossils or not.

The literature survey has been extended to the neighbouring provinces Schleswig in W. Germany (BRD) and Scania, Halland, and Blekinge in Sweden, because the soil and climate do not differ much from what can be found within the borders of present-day Denmark. Furthermore, through most of the Middle Ages these areas were either part of or closely associated with the Kingdom of Denmark.

Of importance in this connection has also been that extensive macrofossil studies already exist from these areas, for instance from Haithabu (in Danish: Hedeby) (Behre 1969, 1978, 1981, 1983) and Lund (Hjelmqvist 1963).

Norway, the Faroe Islands, and Greenland have not been included, since climate, soil, and vegetational history are different in those areas. 


\section{Previous macrofossil surveys}

In Northern Europe the content of macrofossils in ancient soil layers has been revealed through a rather large number of studies. Several of these have been summarized in either national or regional bibliographies.

Information on finds of macrofossils from the area covered by this publication is, for example, contained in "Vor- und frühgeschichtliche Kulturpflanzenfunde in Mitteleuropa" (Willerding 1970) and "Bibliographie zur Paläo-Ethnobotanik des Mittelalters in Mitteleuropa 1945-1977" (Willerding 1978a, 1979a), Hjelmqvist's descriptions of the oldest history of cultivated plants in Sweden (1955, 1979), and "Bibliography and Index to Palaeobotany and Palynology 1950-1970" (Tralau 1974).

Surveys of the flora of Denmark in the Late Weichselian and Flandrian periods (cf. Fig. 1) have been published by Hartz (1902), and Iversen (1954).

The comprehensive survey of the history of the Danish field weeds, published by Jessen \& Lind (1922-23), was an important step forward. This work was supplemented by Helbæk's (1954) survey of archaeobotanical research from 1923 to 1954.
Jessen (1920) presented an important list of plant remains found in post-glacial freshwater strata in NorthEast Zealand.

Willerding (1979b) has discussed agriculture in the Pre-Roman Iron Age in Germany, Denmark, and South Sweden and registered the finds of cultivated species.

The history of the introduction of garden plants into Denmark has been covered by Lange $(1975,1979)$ and Olsen $(1976,1980)$ and in other papers in "Fra Kvangård til Humlekule", a periodical published by The Danish Historic Horticultural Society.

Through germination experiments with archaeologically dated soil samples from c. 200 A.D., and from the Middle Ages and later, Ødum $(1965,1978)$ has demonstrated the occurrence of a number of species from these periods in Denmark and Scania.

In the topographical-botanical descriptions of the Danish flora that have been published in the period 1931-1981 in 'Botanisk Tidsskrift', attempts have been made to establish when the species were first present in Denmark. For references to specific families, see Hansen (1981). 


\section{The catalogue}

\section{Materials}

The catalogue aims to present references to all relevant published finds of macrofossils from Denmark, Schleswig, Scania, Halland, and Blekinge, dated to periods between 13,000 B.P. and 1536 A.D. The term 'macrofossils' is here taken to include carbonized and uncarbonized seeds and fruits, imprints in pottery, burned daub, etc., together with vegetable parts such as leaves, stems, and bud scales. As the chance of transportation of macrofossils from distant areas is usually limited, it is generally assumed that a find means that the species at that time was established in the area.

Finds of wood, charcoal, and wooden artifacts have, however, not been included, as there is a greater likelihood that such macrofossils may have been transported to the site from afar.

During registration it was obvious that certain finds have been cited by several authors. In other cases the publication does not make clear exactly which of the macrofossils from a site is meant. However, no attempt has been made to exclude any publication, even if it contained information that was already present elsewhere.

\section{Tables and figures}

The information from a large number of publications has been arranged in three tables and a map.

The map (Fig. 2) shows the position of each find in Southern Scandinavia, and gives the site number used for it in Tables 1 and 2 . The first digit(s) indicates the location of the site:

$\begin{array}{ll}\text { 1: } & \text { North Jutland } \\ \text { 2: } & \text { South Jutland } \\ \text { 3: } & \text { Schleswig } \\ \text { 4: } & \text { Funen } \\ \text { 5: } & \text { Zealand and Møn } \\ \text { 6: } & \text { Lolland and Falster } \\ \text { 7: } & \text { Bornholm } \\ \text { 8: } & \text { Halland and Blekinge } \\ \text { 9, 10: } & \text { Scania }\end{array}$

The two last digits in the site numbers are sequence numbers used within the areas mentioned above.
Table 1 lists the material by site numbers, adding the name of the site, where it is published, its age, the dating method applied, and which medium was involved.

Table 2 arranges the recorded species/genera alphabetically and gives the appropriate site numbers period by period. When the author considered a determination to be unreliable, the name of the macrofossil is marked 'cf.' and listed last.

The plant names used in the tables are in accordance with Flora Europaea, vol. 1-5, and the synonyms added are those used in the registered papers.

It is not possible to give exact, comparable figures for the number of macrofossils identified in the works listed, since the size of the samples, the medium examined, and the methods applied all varied from study to study. In order to give some information, however, the site numbers in Table 2 are followed by ' $r$ ', ' + ', or ' $c$ ', representing increasing numbers of macrofossils reported. When a publication contains several figures for a given species/period, it is the symbol representing the highest value that is mentioned in Table 2 .

For each species/genus listed in Table 2, the earliest find indicates the assumed time of establishment in Southern Scandinavia. A number of species were, however, most likely present in the area in earlier periods, but finds of macrofossils are missing so far. A few species may have been recorded as macrofossils (e.g. from import), without being established in the area. Therefore, in order to supplement the first recorded finds from Southern Scandinavia, information has been included on either older or contemporary finds from the neighbouring countries: The British Isles (BI), The Netherlands (NL), German Federal Republic (BRD), German Democratic Republic (DDR), Poland (P), Sweden (S), and Norway (N).

When assessing these results, the heterogeneity of the summarized publications must be kept in mind. They cover a period of more than one hundred years from the very beginning of palaeobotany to finds dated with the most recent techniques. The circumstances under which the macrofossils were found vary: they may have been found during description of a soil profile or during highly specialized examinations for content of macrofossils. The skill in performing the identification work and the technical facilities available vary between authors as well.

Table 3 summarizes by family the finds of macrofossils listed in Table 2 . 


\section{Dating}

In Tables 2 and 3 the finds are classified into nine pollen assemblage zones (I-IX) and the following archaeological/historical periods: Pre-Roman Iron Age (PRIA), Roman Iron Age (RIA), Germanic Iron Age (GIA), Viking Age (VA), Early Middle Ages (EMA), and Late Middle Ages (LMA). The relation between the pollen assemblage zones and chronozones (Mangerud et al. 1974), C-14 years, calender years, and archaeological periods is shown in Fig. 1.

Macrofossils dated to a period earlier than the PRIA (e.g. Bronze Age) are assigned to a pollen assemblage zone, regardless of the dating method actually used.

Finds from PRIA and later, if dated by pollen-analysis only, or if dated to only the Iron Age in general, are listed as pollen zone IX.

If possible, finds are referred to one of the above periods, but in cases where the dating given was on the border between two zones, the finds are here assigned to the younger. This avoids the danger of inflating the age of arrival of plants in our area by giving dates that are too early.

The chronozones Bølling and Older Dryas (cf. Fig. 1) are not separated here, as this was not done in older publications containing valuable information on macrofossils. Finds of macrofossils in these periods are summarized by Iversen (1954).

It appears from Fig. 1, that the chronozone border Preboreal/Boreal and Boreal/Atlantic is not synchronous with Iversen's $(1960,1967,1973)$ zone border IV/V and V/VI. Nor are Iversen's zone borders exactly synchronous with the zones defined by Jessen (1935). The border zone V/VI is defined by Jessen (1.c.) as the rational limit of $A$ lnus, where Iversen (1.c.) prefers the first rise of Tilia in his pollen diagrams. Therefore, Jessen's borders are usually slightly older than those of Iversen.

The zone borders V/VI and VI/VII have also been discussed by Jørgensen (1954). He defined the zone border V/VI as the rational limit of 'Quercetum mixtum', which is somewhat earlier than the rational limit of Alnus (cf. Jessen 1935). As the zone border VI/VII differs between Jessen (l.c.), Iversen (l.c.), and Jørgensen (l.c.), finds referred to zones VI and VII are combined in Tables 2 and 3. In Table 2 the finds from zone VI are listed first.

The zone border VII/VIII of Jessen (1.c.) differs slightly from that of Iversen (l.c.), since Jessen uses the decrease of Tilia, and Iversen the decrease of Ulmus (cf. discussion by Andersen 1978).

The zones defined for Southern Sweden by Nilsson $(1935,1961)$ are also included in Fig. 1. The relation to the zones defined by Jessen (l.c.) is in accordance with Nilsson (1961). The zones used by Wiermann (1962) are related to Jessen's zones by means of the diagram published by Overbeck (1975, Table 24).
The datings to which some doubt attaches are marked with the letter ' $u$ ' (uncertain) in Tables 2 and 3. Finds whose dating is definitely unreliable or altogether lacking have been omitted. This means that in the case of some of the cited publications, only a part of the listed finds are included in the catalogue. In spite of this evaluation, the accuracy of dating may vary between publications. Therefore, the assumed establishment of species in Southern Scandinavia - based on records referred to the oldest period - can in general be considered more certain when supported by more than one find.

The first recorded finds are as mentioned also supported by finds from neighbouring countries (cf. Table 2 ). The dating of these finds is, as far as possible, presented as published. For this reason, some of the C-14 datings are expressed as conventional $\mathrm{C}-14$ years ('B.P.'), others as calendar years ('B.C.' or 'A.D.'). For the latter datings it is, unfortunately, not always possible to ascertain whether the $\mathrm{C}-14$ datings are expressed as $\mathrm{C}-14$ years or corrected to calender years.

Godwin's (1975) pollen assemblage zones, frequently cited from the British Isles, are with some reservations comparable to the zones used by Iversen $(1960,1967)$. Godwin's zones VIIa, VIIb, and VIII correspond to Iversen's zones VII, VIII, and IX, respectively (cf. Fig. 1).

In some papers references are made to:

Band Ceramic: An agrarian neolithic culture, widely distributed in Central and Northern Europe from c. 4500 B.C. (Rud 1979)

Hallstatt Period: C. $700-$ c. 450 B.C. (Rud 1979)

La Têne Period: C. 450 - c. 1 B.C. (Rud 1979)

Due to the possible variation in the interpretation of pollen diagrams, datings, etc., it is recommended that the original publications be consulted before definite conclusions are made.

\section{Medium examined}

Table 1 includes a brief description of the medium examined, the preservation of the macrofossils and the possibilities for their identification being highly influenced by this factor. In characterizing the medium examined, several authors have used the principles defined by Troels-Smith (1955).

Some of the terms used for the medium examined may need an explanation:

Burnt house: House evidently destroyed by fire.

Burnt layer: Excavated layer affected by fire, but without indication of house remains. 
Germination: Archaeologically dated soil samples examined for content of macrofossils by germination.

Grave: $\quad$ Macrofossils recovered from barrows and other graves.

Imprint: Imprint of macrofossils in vessels, potsherds, or clay walls.

Latrine: Macrofossil analyses from latrine deposits.

Manure: $\quad$ Preserved dung mixed with straw and other plant remains.
Pit: $\quad$ Macrofossils found in post holes, pits, and rubbish holes.

Soil: $\quad$ Earth samples - often affected by cultivation - not classified as clay, gyttja, humus, lime, peat, sand, or silt.

Stomach:

Analyses of stomach content from preserved corpses found in peat bogs.

Storage: Grain, etc. stored for later consumption.

Vessel: $\quad$ Plant remains found in vessels of bronze or clay. 


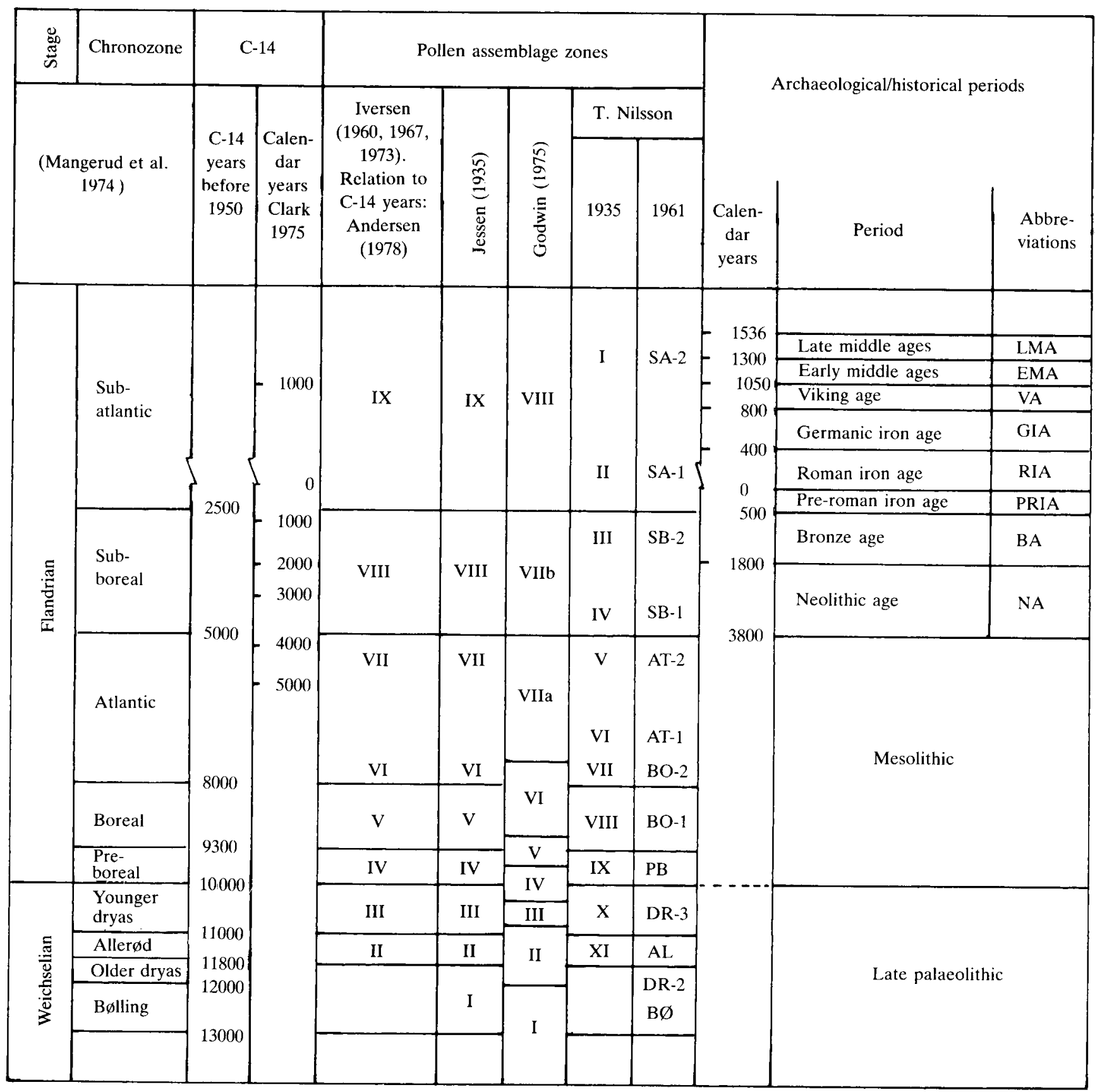

Fig. 1. Relation between periods and dating systems. 



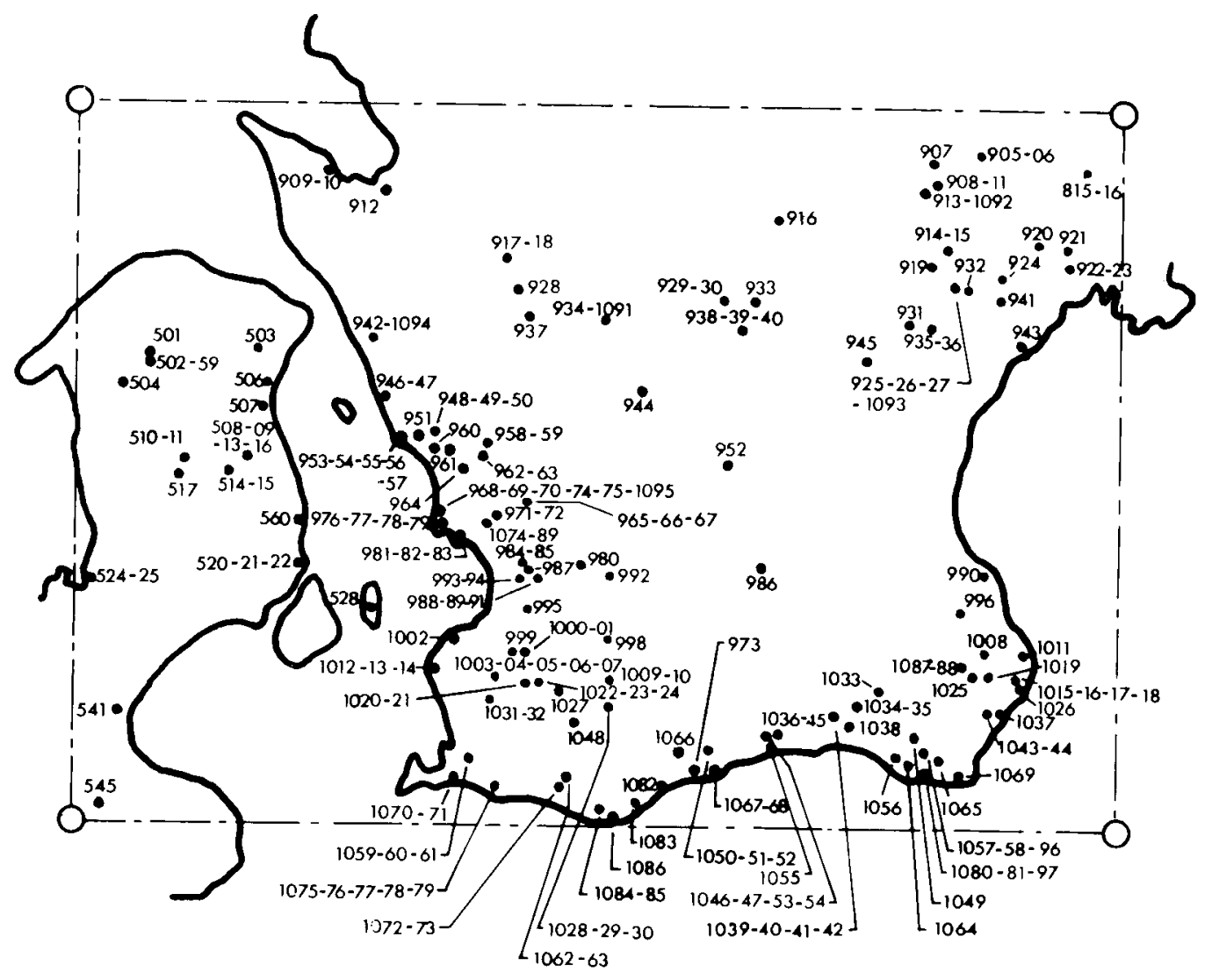

Fig. $2 A$. 


\section{Concluding remarks}

The information on finds of macrofossils assembled in this catalogue invites further study of the macrofossils identified from different periods.

It appears from the tables that the families, the periods, and the media examined are unequally repre- sented, owing to inequalities of research, differential macrofossil preservation, problems of identification, etc.

It is intended to discuss these subjects further in a forthcoming publication. 


\section{Acknowledgments}

Through the long task of collecting literature, discussion of materials and preparation of the manuscript, I have received valuable aid from many colleagues in and outside Denmark. For the loan of reprint collections and extensive advice I am especially indebted to Dr. phil. Svend Th. Andersen and Cand. scient. Bent Odgaard, Geological Survey of Denmark; Dr. phil. Bent Fredskild, University of Copenhagen; Professor, Dr. phil. Vald. M. Mikkelsen, The Royal Veterinary and Agricultural University; and to Dr. phil. J. TroelsSmith, The Danish National Museum. Assistant Tove Due Andersen, Stud. scient. Jan C. van Dijk, Librarian Christel M. Erschens, Cand. hort. Maja From, and
Assistant Else Sørensen have with care and enthusiasm participated in registration and preparation of the manuscript. My colleague, Dr. Ole B. Lyshede, has through numerous discussions made a valuable contribution to the work.

The registration of the literature was supported by grant number 11-3357 from the Danish Natural Science Research Council.

The printing has been supported by grant number 13-3403 from the Danish Agricultural and Veterinary Research Council.

For every aid and support received I wish to extend my sincere thanks. 
Andersen, A. 1975. Geologiske unders $\varnothing$ gelser omkring Borremosebebyggelsen. - Årb. N.O.: 96-119.

- \& Møller, K. 1946. Fund af urokse (Bos taurus urus L.) i Grænge Mose på Lolland. - Danm. geol. Unders. IV. række 3 (1): 1-17.

Andersen, N. H. 1980. Sarup. Befæstede neolitiske anlæg og deres baggrund. - Kuml 1980: 63-103.

Andersen, S. T. 1961. Vegetation and its environment in Denmark in the Early Weichselian Glacial (Last Glacial). - Danm. geol. Unders. II. rakke 75: 1-175.

- 1978. Local and regional vegetational development in eastern Denmark in the Holocene. - Danm. geol. Unders. Årbog 1976: 5-27.

- 1984. Forests at Løvenholm, Djursland, Denmark, at present and in the past. - Biol. Skr. Dan. Vid. Selsk. 24 (1): 1-208.

- , Mortensen, P., Olsen, O., Steensberg, A. \& Troels-Smith, J. 1983. Kommissionen til udforskning af Danmarks ældste landbrug og de samtidige naturforhold. - Kgl. Dsk. Vid. Selsk. - Oversigt over selskabets virksomhed 1982/83: 95-97.

Averdieck, F.-R. 1980. Zur Vegetations- und Siedlungsgeschichte bei Bosau. - In: Hinz, H. (ed.), Bosau. Untersuchung einer Siedlungskammer in Ostholstein IV. Offa-Bücher 42, pp. 97-106.

- 1981. Paläobotanische Untersuchungen an Wallproben von Alt Lübeck. - LSAK 5: 103-111.

- Eberle, G. \& Willkomm, H. 1982. Der "Buchenwaldtorf" vom Dummersdorfer Ufer bei Lübeck-Travemünde. - Abh. Naturw. Verein Bremen 39: 299-311.

Behre, K.-E. 1969. Untersuchungen des botanischen Materials der frühmittelalterlichen Siedlung Haithabu. - In: Schietzel, K. (ed.), Berichte über die Ausgrabungen in Haithabu. Bericht 2, Karl Wachholtz Verlag, Neumünster, pp. 7-55.

- 1975. Wikingerzeitlicher Ackerbau in der Seemarsch bei Elisenhof/Schleswig-Holstein (Deutsche Nordseeküste). - Folia Quatern. 46: 49-62.

- 1976. Die Pflanzenreste aus der frühgeschichtlichen Wurt Elisenhof. - Studien zur Küstenarchäologie Schleswig-Holsteins, Serie A. Elisenhof: Die Ergebnisse der Ausgrabung beim Elisenhof in Eiderstedt 1957/58 und 1961/64. Bd. 2. Herbert Lang, Bern.

- 1978. Formenkreise von Prunus domestica L. von der Wikingerzeit bis in die frühe Neuzeit nach Fruchtsteinen aus Haithabu und AltSchleswig. - Ber. Deutsch. Bot. Ges. 91: 161-179.

- 1981. Zur Nahrungswirtschaft der Wikingerzeit im nördlichen Mitteleuropa. - Z. Archäol. 15: 25-40.

- 1982. Zwei jungbronzezeitliche Getreidefunde aus Niedersachsen. - NNU 51: 281-292.

- 1983. Ernährung und Umwelt der wikingerzeitlichen Siedlung Haithabu. Die Ergebnisse der Untersuchungen der Pflanzenreste. - Karl Wachholtz Verlag, Neumünster.

Berglund, B. E. 1964. The post-glacial shore displacement in eastern Blekinge, southeastern Sweden. - Sver. geol. Unders. Ser. C nr. 599. Årsbok 58 (5): 1-47.

- 1966a. Late-quaternary vegetation in Eastern Blekinge, SouthEastern Sweden. A pollen-analytical study. I. Late-glacial time. Op. Bot. Soc. Bot. Lund. 12 (1): 1-190.

- 1966b. Late-quaternary vegetation in Eastern Blekinge, SouthEastern Sweden. A pollen-analytical study. II. Post-glacial time. Op. Bot. Soc. Bot. Lund. 12 (2): 1-180.

- \& Digerfeldt, G. 1970. A palaeoecological study of the Late-Glacial lake at Torreberga, Scania, South Sweden. - Oikos 21: 98-128.
Bertsch, K. 1927. Die Obstreste aus den Alamannengräbern von Oberflacht. - Ber. Deutsch. Bot. Ges. 45: 23-30.

- 1954. Vom neolithischen Feldbau auf der Schwäbischen Alb. Ber. Deutsch. Bot. Ges. 67: 18-21.

Björk, S. \& Digerfeldt, G. 1965. Notes on the limnology and PostGlacial development of Lake Trummen. - Bot. Notiser 118 (3): 305-325.

Brandt, I. 1951. Planterester i et moselig fra Borremose. - Årb. N.O. 1950: 342-351

- 1954. Late-glacial macroscopic plant remains from Böllingsö. Danm. geol. Unders. II. række 80: 156-158.

Broholm, H. C. 1938. Jelsfundet. En sønderjydsk mandsgrav fra den ældre bronzealder. - Aarb. N.O. 1938: 1-20.

Brøndsted, J. 1938. Danmarks oldtid, vol. I. - Nordisk Forlag, København.

- 1940. Danmarks oldtid, vol. III. - Nordisk Forlag, København.

Clark, R. M. 1975. A calibration curve for radiocarbon dates. - Antiquity $49: 251-266$.

Degerbøl, M. \& Iversen, J. 1945. The bison in Denmark: A zoological and geological investigation of the finds in Danish Pleistocene deposits. - Danm. geol. Unders. II. række 73: 1-62.

Dickson, J. H., Dickson, C. A. \& Breeze, D. J. 1979. Flour or bread in a Roman military ditch at Beardsden, Scotland. - Antiquity 53: 47-51.

Digerfeldt, G. 1966. Utvecklingshistoriska och limnologiska observationer i Ranviken av sjön Immeln. - Bot. Notiser 119 (2): 216-232.

- 1974. The post-glacial development of the Ranviken bay in Lake Immeln. - Geol. För. Stockh. Förh. 96: 3-32.

Feindt, F. 1975. Vorläufiger Bericht über einen Fund von verkohltem Getreide aus einem kaiserzeitlichen Haus auf der nordfriesischen Insel Amrum. - Folia Quartern. 46: 41-48.

Fischer, C. 1980. Moseligene fra Bjældskovdal. - Kuml 1979: 7-44. Fredskild, B. 1971. Makroskopiske planterester fra det aldste Århus. - In: Andersen, H. H., Crabb, P. J. \& Madsen, H. J. (eds.), Århus Søndervold: en byarkæologisk undersøgelse. Jysk Arkæologisk Selskabs Skrifter Bd. IX. Nordisk Forlag, København, pp. 307-318.

- 1975. A late-glacial and early post-glacial pollen-concentration diagram from Langeland, Denmark. - Geol. För. Stockh. Förh. 97: 151-161.

- 1977. Blommestenene fra Langeland. - Urt 1977: 25-26.

- 1979. En mosegeologisk undersøgeise af Flaadet-bopladsen. - In: Skaarup, J. (ed.), Flaadet. En tidlig maglemoseboplads på Langeland. Langelands Museum, Rudkøbing, pp. 17-34.

Frödin, O. 1910. En svensk pålbyggnad från stenåldern. - Fornvännen 5: 29-77.

Geel, B. van, Hallewas, D. P. \& Pals, J. P. 1983. A late Holocene deposit under the Westfriese zeedijk near Enkhuizen (prov. of Noord-Holland, The Netherlands): Palaeoecological and archaeological aspects. - Rev. Palaeobot. Palynol. 38: 269-335.

Gluza, I. \& Wasylikowa, K. 1977. Flora of the Pleistocene and the Holocene in archaeological excavations. - In: Sokolowski, S. (ed.), Geology of Poland, Vol. 2, Part 3b, pp. 105-122.

Godwin, H. 1975. The history of the British flora, ed. 2. - The University Press, Cambridge.

Gram, B. 1911. Mikroskopiske undersøgelser. - In: Müller, S. (ed.), Juellingefundet og den romerske periode. Nordiske Fortidsminder II, 1. Gyldendalske Boghandel, København, pp. 40-46. 
Green, F. J. 1978. Plant remains. - In: Heighway, C. M., Garrod, A. P. \& Vince, A. G. (eds.), Excavations at I Westgate Street, Gloucester, (Appendix 5). Medieval Archaeology 22, pp. 186-190.

Greig, J. R. A. 1976. The plant remains.--In: Buckland, P. C. (ed.), The Environmental Evidence from the Church Street Roman Sewer System. The Past Environment of York 14 (1), pp. 23-28.

- 1979a. Seeds and pollen from site SK187082. - In: Smith, C. (ed.), Fisherwick: the reconstruction of an Iron Age landscape. British Archaeological Reports, British Series, No. 61, Oxford, pp. 81-84.

- 1979b. Seeds and pollen. - In: Smith, C. (ed.), Fisherwick: the reconstruction of an Iron Age landscape. British Archaeological Reports, British Series, No. 61, Oxford, pp. 185-188.

- 1980. Seeds from the Well. - In: Stead, I. M. (ed.), Rudston Roman Villa. Yorkshire Archaeological Society, Leeds, pp. 169-171.

Griffin, K. O. 1975. Macrofossils from Gamlebyen, an archaeological site in Oslo, Norway: A preliminary report. - Folia Quaternaria (Krakow) 46: 63-67.

- 1977. Plant remains from Medieval Oslo. - Universitetets Oldsaksamling. Årbok 1975/1976, pp. 151-163.

- 1979a. Plant remains from "Oslogate 7". - De Arkeologiske Utgravninger i Gamlebyen, Oslo 2: 124-133.

- 1979b. Fossil records of fig, grape and walnut in Norway from Medieval Time. - Archaeo-Physika 8: 57-67.

- 1980. A subfossil find of Ceratophyllum submersum in Norway. Norw. J. Bot. 27: 207-217.

- 1981. Plant remains from archaeological sites in Norway: a review. - Z. Archäol. 15: 163-176.

- 1982. Utgrävningen på Helgeandsholmen. Analyser av jordprover från 7 båtar. - Raä 2: 1-25.

Grönwall, K. A. \& Milthers, V. 1916. Beskrivelse til geologisk kort over Danmark. Kortbladet Bornholm. - Danm. geol. Unders. I. rakke 13: 1-281.

Hansen, K. (ed.) 1981. Dansk feltflora. - Gyldendal, Copenhagen.

Hartz, N. 1902. Bidrag til Danmarks senglaciale flora og fauna. Danm. geol. Unders. II. række 11: 1-80.

- \& Milthers, V. 1901. Det senglaciale ler i Allerød Teglværksgrav. Meddr. Dansk geol. Foren. 8:31-60.

- \& Winge, H. 1906. Om uroksen fra Vig, såret og dræbt med flintvåben. - Aarb. N.O. II. række 21: 225-236.

Hatt, G. 1930. En brandtomt af et jernaldershus på Mors. - Aarb. N.O. 1930, III. række 20: 83-118.

- 1935. En jernalders hustomt i Troldtoft, Vind Sogn. - Aarb. N.O. 1935: 47-57.

- 1937. Landbrug i Danmarks oldtid. - G. E. C. Gad, København.

- 1938. Jernalders bopladser i Himmerland. - Aarb. N.O. 1938: 119-266.

- 1948. En boplads fra germansk jernalder i Oksbøl. - Fra Ribe Amt XII (I): 36-59.

- 1949. Oldtidsagre. - Kgl. Dan. Vid. Selsk. Arkæol.-Kunsth. Skr. II (I): 1-182.

- 1959. Iron Age cellars at Bækmoien, North Jutland. - Acta Archaeologica XXX: 201-216.

- 1960. The Roman Iron Age dwelling site at Mariesminde, Vestervig. - Acta Archaeologica XXXI (I): 63-83.

Helbæk, H. 1950. Tollund mandens sidste måltid. - Årb. N.O. 1950: 311-341.

- 1951. Ukrudtsfrø som næringsmiddel i førromersk jernalder. Kuml 1951: 65-74.

- 1952a. Spelt (Triticum spelta L.) in Bronze Age Denmark. - Acta Archaeologica XXIII: 97-107.

- 1952b. Preserved apples and Panicum in the prehistoric site at Nørre Sandegård in Bornholm. - Acta Archaeologica XXIII: 107115.

- 1952c. Early crops in Southern England. - Proc. Prehist. Soc. 1952 (12): 194-233.

- 1953. Sæddodder og korn på Bornholm i det 13. århundrede. Bornholmske Samlinger XXXIII: 9-15.

- 1954. Prehistoric food plants and weeds in Denmark. A survey of archaeobotanical research 1923-1954. - Danm. geol. Unders. II. række 80: 250-261.

- 1955a. Store Valby - kornavl i Danmarks første neolitiske fase. Årb. N.O. 1954: 198-204.

- 1955b. The botany of the Vallhagar Iron Age Field. - In: Stenberger, M. (ed.), Vallhagar. II, pp. 653-799. Munksgaard, Copenhagen.

- 1957. Bornholm plant economy in the first half of the first millennium A.D. - In: Bornholm i folkevandringstiden. Nationalmuseets Skrifter, Større Beretninger II, pp. 259-277.

- 1958a. Grauballemandens sidste måltid. - Kuml 1958: 83-116.

- 1958b. The Oxbøl grain. - Acta Archaeologica XXIX: 155-157.

- 1958c. Korndyrkningens udvikling på Bornholm. - Bornholmske Samlinger 36: 117-129.

- 1959. Notes on the evolution and history of Linum. - Kuml 1959: 103-129.

- 1964. The Isca grain, a Roman plant introduction in Britain. - New Phytol. 63: 158-164.

- 1974. The Fyrkat grain. A geographical and chronological study of rye. In: Fyrkat. En jysk vikingeborg. - Nordiske Fortidsminder, serie B, 2, pp. 1-41.

Hinz, H. 1951. Eine Siedlung der Kaiserzeit von Westerohrstedt. Offa $9: 57-62$

- 1954. Kaiserzeitliche Samen aus Westerohrstedt, Kreis Husum (Nachtrag). - Offa 13: 87-88.

Hjelmqvist, H. 1952. Några sädeskornavtryck från Sydsveriges stenålder. - Bot. Notiser 1952 (3): 330-338.

- 1953. Ett bidrag till kulturväxternas äldsta historia i Sydsverige. Bot. Notiser 1953 (4): 420-430.

- 1955a. Die älteste Geschichte der Kulturpflanzen in Schweden. Op. Bot. Soc. Bot. Lund 1 (3): 1-186.

- 1955b. Kulturväxternas äldsta historia i Sverige. - St. naturvet. forskningsråds Årsb. 1955: 286-297.

- 1958a. Cereal impressions from the Early-Neolithic habitation at Vätteryd. - Meddn. Lunds Univ. Hist. Mus. 1958: 103-106.

- 1958b. Fynd av vete från Skånes tidig-neolitiska tid. - Bot. Notiser 111 (1): 399-400.

- 1960. Några växtfynd från Sveriges järnålder. - Bot. Notiser 113 (2): 141-160.

- 1962. Getreideabdrücke in der Keramik der schwedisch-norwegischen Streitaxtkultur. - Acta Archaeologica Lundensia. Ser. in $8^{\circ}$, no 2: 911-912.

- 1963. Frön och frukter från det äldsta Lund. - Archaeologica Lundensia II (10): 233-270.

- 1964. Kulturväxter från Skånes forntid. - Ale. Hist. Tidsskr. för Skåneland 1: 23-35.

- 1968a. Einige Mittelalterliche Pflanzenfunde. - Archaeologica Lundensia III. Res Mediaevales: 179-194.

- 1968b. Getreideabdrücke in der neolithischen Keramik des Dolmens Trollasten. - Acta Archaeologica Lundensia. Ser. in $8^{\circ}$, no 7 : 243-249.

- 1969a. Dinkel und Hirse aus der Bronzezeit Südschwedens nebst einigen Bemerkungen über ihre spätere Geschichte in Schweden. Bot. Notiser 122: 260-270.

- 1969b. Getreideabdrücke in den bronzezeitlichen Funden aus Hötofta. - Acta Archaeologica Lundensia. Ser. in 8, no 8: 208-216.

- 1970. Getreideabdrücke in der frühneolithischen Keramik von Värby. - Acta Archaeologica XLI: 96-98.

- 1971-72. Ein Beitrag zur Kenntnis der Nutzpflanzen am Übergang von Stein- zur Bronzezeit in Schonen. - Meddn. Lunds Univ. Hist. Mus. 1971-72: 144-150.

- 1972. Ett sädeskornsfynd från forna tiders Halland. - Halland 1972 (Hallands Museum, Halmstad): 70-75.

- 1974. Früchte und Samen von der frühneolithischen Siedlung bei Sturup. - In: Christofferson, K. (ed.), Inventering och arkeologisk undersökning 1969-1971: Sturupområdet, Börringe och Genarps socknar, Skåne. Riksantikvarieämbetets Rapport 1974, B 35, pp. 215-220. 
Hjelmqvist, H. 1975a. Några vittnesbörd om sädesodlingen på Jylland i äldre romersk järnålder. - Kuml 1973-74: 209-214.

1975b. Getreidearten und andere Nutzpflanzen aus der frühneolithischen Zeit von Langeland. - In: Skaarup, J. (ed.), Stengade: Ein langeländischer Wohnplatz mit Hausresten aus der frühneolithischen Zeit, Langelands Museum, Rudkøbing, pp. 211-219.

- 1979. Beiträge zur Kenntnis der prähistorischen Nutzpflanzen in Schweden. - Opera Bot. 47: 1-58.

- 1981. Grain impressions from Gårdlösa. - In: Stjernquist, B. (ed.), Gårdlösa. An Iron Age community in its natural and social setting. Acta Regiae Societatis Humaniorum Litterarum Lundensis LXXV, pp. 54-58.

- 1981-82. Economic plants from a Middle Neolithic site in Scania. Medd. Lunds universitets historiska Museum 1981-1982: 108-113.

Iversen, J. 1934. Fund af vildhest (Equus caballus) fra overgangen mellem sen- og postglacialtid i Danmark. - Danm. geol. Unders. IV. række 2 (13): 3-16.

- 1939. Planterester fremdragne i tre høje i Haderslev Amt. - Nordiske Fortidsminder III (2): 18-21.

- 1946. Geologisk datering af en senglacial boplads ved Bromme. Aarb. N.O. 1946: 198-230.

- 1954. The Late-Glacial flora of Denmark and its relation to climate and soil. - Danm. geol. Unders. II. række 80: 87-119.

- 1960. Problems of the early post-glacial forest development in Denmark. - Danm. geol. Unders. IV. rakke 4 (3): 1-32.

- 1967. Naturens udvikling siden sidste istid. - In: Nørrevang, A. \& Meyer, T. J. (eds.), Danmarks Natur vol. 1. Politikens Forlag A/S, pp. $345-445$.

- 1973. The development of Denmark's nature since the Last Glacial. Transl. from Danmarks Natur, vol. 1: 345-445, 1967 by M. Robson. Ed. by S. T. Andersen, Danm. geol. Unders. V. række 7c: 1-126.

Jäger, K.-D. 1965. Verkohlte Samen aus einem bronzezeitlichen Grabgefäss von Tornow, Kr. Calau. - Nachrichtenbl. Vor- und Frühgesch. 10 (3): 131-138.

- 1966. Die pflanzliche Grossreste aus der Burgwallgrabung Tornow, Kr. Calau. - In: Hermann, J. (ed.), Tornow und Vorberg. Ein Beitrag zur Frühgeschichte der Lausitz. Berlin, pp. 164-189.

Jensen, H. A. 1979a. Seeds and other diaspores in Medieval layers from Svendborg. - The Archaeology of Svendborg 2: 1-102.

- 1979b. Frøundersøgelser fra det middelalderlige Svendborg. Skrifter fra Svendborg \& Omegns Museum 3: 46-74.

- Under preparation. Seeds and other diaspores in samples from Danish town and monastery excavations, dated 700-1536 A.D. Biol. Skr. Dan. Vid. Selsk. 26.

Jessen, A. 1935. Beskrivelse til geologisk kort over Danmark. Kortbladet Haderslev. - Danm. geol. Unders. I. række 17: 1-95.

Jessen, K. 1916. Et urokse-fund på Lørup Hede. Foredrag. - Vidensk. Meddr. Dansk Naturh. Foren. 67: XVII-XVIII.

- 1919. Mindre meddelelser om fortidens plantevækst i Danmark. Bot. Tidsskr. 36: 51-56.

- 1920. Moseundersøgelser i det nordøstlige Sjælland. - Danm. geol. Unders. II. række 34: 1-268.

- 1923. En undersøisk mose i Rungsted Havn og senglaciale niveauforandringer i Øresund. - Danm. geol. Unders. IV. række 1 (18): $1-18$.

- 1924a. Et bjørnefund i Allerødgytje. - Meddr. dansk geol. Foren. 6 (24): $1-11$

- 1924b. De geologiske forhold ved de to bopladser i Holmegårds Mose. - In: Broholm, H. C. (ed.), Nye fund fra den ældste stenalder, Holmegård og Sværdborgfundene. Aarb. N.O. III. række 14, pp. 14-27.

- 1927. Et kulturlag fra den ældre stenalder ved Hors $\emptyset$. De geologiske forhold. - Meddr. Dansk geol. Foren. 7 (2): 129-138.

- 1929. Senkvartære studier fra Mors. - Danm. geol. Unders. IV. række 2 (5): 1-22.

- 1933. Planterester fra den ældre jernalder i Thy. - Bot. Tidsskr. 42 (3): $257-288$
- 1935a. Archaeological dating in the history of North Jutland's vegetation. - Acta Archaeologica V: 185-214.

- 1935b. The composition of the forests in Northern Europe in Epipalæolithic time. - Kgl. Dan. Vid. Selsk. Biol. Meddr. XII (1): 1-64.

- 1937. Den geologisk-botaniske undersøgelse af Hjortspring Mose. - Nordiske Fortidsminder III (1): 25-32.

- 1938a. Some west Baltic pollen diagrams. - Quartär 1: 124-139.

- 1938b. II. Bundsøkulturens pollendiagrammatiske stilling. - Aarb. N.O. 1938: 56-64.

- 1939a. Senglaciale planteførende ferskvandsaflejringer. - In: Milthers, V. (ed.), Beskrivelse til geologisk kort over Danmark. Kortbladet Brande. Danm. geol. Unders. I. rakke 18, pp. 79-80.

- 1939b. III. Kornfund. - Aarb. N.O. 1939: 65-84.

- 1939c. Postglaciale aflejringer. - In: Milthers, V. (ed.), Beskrivelse til geologisk kort over Danmark. Kortbladet Brande. Danm. geol. Unders. I. række 18, pp. 128-143.

- 1948. Om naturforholdene ved Trelleborg og forbruget af træ ved borgens opførelse. - In: Nørlund, P. (ed.), Trelleborg. Nordiske Fortidsminder IV (1), pp. 163-179.

- 1954. Plantefund fra vikingetiden i Danmark. - Bot. Tidsskr. 50: 125-139.

- \& Helbæk, H. 1944. Cereals in Great Britain and Ireland in Prehistoric and Early Historic Times. - Kgl. Dan. Vid. Selsk. Biol. Skr. III (2): $1-68$.

- \& Lind, J. 1922-23. Det danske markukrudts historie. - Kgl. Dan. Vid. Selsk. Skr. Naturvid. Math. Afd. 8. række VIII.

- \& Milthers, V. 1928. Stratigraphical and paleontological studies of interglacial fresh-water deposits in Jutland and Northwest Germany. - Danm. geol. Unders. II. rakke 48: 1-379.

Jonassen, H. 1936. Et pollendiagram fra Karupfladen. - Bot. Tidsskr. 43 (3): 187-196.

- 1950. Recent pollen sedimentation and Jutland heath diagrams. Dansk Bot. Ark. 13 (7): 1-168.

- 1954. Dating of sand-drift east of Ulfborg. - Bot. Tidsskr. 51: 136140.

Jørgensen, G. 1977a. Acorns as a food-source in the Later Stone Age. - Acta Archaeologica 48: 233-238.

- 1977b. Et kornfund fra Sarup. Bidrag til belysning af tragtbægerkulturens agerbrug. - Kuml 1976: 47-64.

- 1979. A new contribution concerning the cultivation of spelt, Triticum spelta L., in prehistoric Denmark. - Archaeo-Physika 8: 135-145.

- 1980. Om kostvaner i det middelalderlige Svendborg. - Naturens Verden 1980: 203-209.

- \& Fredskild, B. 1978. Plant remains from the TRB culture, period MN V. - In: Davidsen, K. (ed.), The final TRB culture in Denmark. Arkæologiske Studier V, pp. 189-192.

Jørgensen, S. 1954. A pollen analytical dating of Maglemose finds from the bog Åmosen, Zealand. - Danm. geol. Unders. II. række 80: $159-187$.

- 1956. Grauballemandens fundsted. - Kuml 1956: 114-130.

- 1963. Early postglacial in Åmosen. Geological and pollenanalytical investigations of Maglemosian settlements in the West-Zealand bog Åmosen. - Danm. geol. Unders. II. række 87 (II): 1-36.

Kenward, H. K. \& Williams, D. 1979. Biological evidence from the Roman Warehouses in Coney Street. - In: Addyman, P. V. (ed.), The Archaeology of York, Vol. 14 (2), pp. 45-100.

Knörzer, K.-H. 1967a. Untersuchung der Lagerungsverhältnisse von Pflanzenresten in einer römerzeitlichen Grube bei Neuss/Rh. - In: Knörzer, K.-H. (ed.), Untersuchungen subfossiler pflanzlicher Grossreste im Rheinland. Archaeo-Physika 2, pp. 76-99.

- 1967b. Römerzeitliche Pflanzenfunde aus Aachen. - In: Knörzer, K.-H. (ed.), Untersuchungen subfossiler pflanzlicher Grossreste im Rheinland. Archaeo-Physika 2, pp. 39-64.

- 1967c. Der römerzeitliche Heilkräuterfund aus Neuss/Rh. - In: Knörzer, K.-H. (ed.), Untersuchungen subfossiler pflanzlicher Grossreste im Rheinland. Archaeo-Physika 2, pp. 65-75. 
Knörzer, K.-H. 1967d. Subfossile Pflanzenreste von bandkeramischen Fundstellen im Rheinland. - In: Knörzer, K.-H. (ed.), Untersuchungen subfossiler pflanzlicher Grossreste im Rheinland. Archaeo-Physika 2, pp. 3-29.

- 1968. Ein Teilergebnis der Untersuchung pflanzlicher Grossreste bei der Ausgrabung an der Niederungsburg bei Haus Meer, Gemeine Büderich. - Rheinische Ausgrabungen 1: 97-100.

1970. Römerzeitliche Pflanzenfunde aus Neuss. - Novaesium IV. (Limesforschungen 10). Verlag Gebr. Mann, Berlin.

- 1971a. Pflanzliche Grossreste aus der rössenerzeitlichen Siedlung bei Langweiler, Kreis Jülich. - Bonner Jahrb. 171: 9-33.

- 1971b. Prähistorische Mohnsamen im Rheinland. - Bonner Jahrb. 171: 34-39.

- 1971c. Eisenzeitliche Pflanzenfunde im Rheinland. - Bonner Jahrb. 171: 40-58.

- 1973. Beiträge zur Frühgeschichte des Obstes im Rheinland. Acta Mus. Agric. Pragae 6-8: 43-53.

- 1974a. Bandkeramische Pflanzenfunde von Bedburg-Garsdorf, Kreis Bergheim/Erft. - Rheinische Ausgrabungen 15: 173-192.

- 1974b. Eisenzeitliche Pflanzenfunde aus Frixheim-Anstel, Kreis Grevenbroich. - Rheinische Ausgrabungen 15: 405-414.

- 1975. Mittelalterliche und jüngere Pflanzenfunde aus Neuss am Rhein. - ZAM 3: 129-181.

- 1977. Pflanzliche Grossreste des bandkeramischen Siedlungsplatzes Langweiler 9. - In: Kuper, R., Löhr, H., Lüning, J., Stehli, P. \& Zimmermann, A. (eds.), Der bandkeramische Siedlungsplatz Langweiler 9, Gemeinde Aldenhaven, Kreis Düren, pp. 279-304. Rheinland-Verlag GmbH, Bonn.

- 1979a. Pflanzliche Grossreste des latènezeitlichen Siedlungsplatzẹs Grevenbroich-Gustorf, Kreis Neuss. - Rheinische Ausgrabungen 19: $601-610$.

- 1979b. Verkohlte Reste von Viehfutter aus einem Stall des römischen Reiterlagers von Dormagen. - Rheinische Ausgrabungen 20: $130-137$.

Körber-Grohne, U. 1967. Geobotanische Untersuchungen auf der Feddersen Wierde. - Feddersen Wierde Bd. 1, Wiesbaden.

- 1979a. Samen, Fruchtsteine und Druschreste aus der Wasserburg Eschelbronn bei Heidelberg. - Forsch. Ber. Archäol. Mittelalters Baden-Württemberg 6: 113-127.

- 1979b. Einige allgemeine Bemerkungen zu einer pflanzensoziologischen Zuordnung subfossiler Floren des Postglazials. - In: Wilmanns, O. \& Tüxen, R. (eds.), Werden und Vergehen von Pflanzengesellschaften, pp. 43-59.

- 1981. Pflanzliche Abdrücke in eisenzeitlicher Keramik - Spiegelbild damaliger Nutzpflanzen? - Fundb. Baden-Württemberg 6 : 165-211.

- \& Piening, U. 1979. Verkohlte Nutz- und Wildpflanzenreste aus Bondorf, Kreis Böblingen. - Fundb. Baden-Württemberg 4: 152169.

Kosina, R. 1978. The cultivated and wild plants from the XIth century granaries on the cathedral-island in Wroclaw. - Ber. Deutsch. Bot. Ges. 91: 121-127.

Krog, H. 1954. Pollen analytical investigations of a C-14-dated Alleröd-section from Ruds-Vedby. - Danm. geol. Unders. II. række 80: $120-139$.

Kroll, H. J. 1975. Ur- und Frühgeschichtlicher Ackerbau in Archsum auf Sylt. Eine botanische Grossrestanalyse. - Dissertation, Christian-Albrechts-Universität, Kiel.

- 1976. Pflanzliche Abdrücke in der Keramik der befestigten neolithischen Siedlung Büdelsdorf, Kreis Rendsburg-Eckernförde. Offa 33: 61-65.

- 1978. Kirschfunde aus dem 13./14. bis 16. Jahrhundert aus der Lübecker Innenstadt. - Ber. Deutsch. Bot. Ges. 91: 181-185.

- 1980. Einige vorgeschichtliche Vorratsfunde von Kulturpflanzen aus Norddeutschland. - Offa 37: 372-383.

- 1981. Mittelneolithisches Getreide aus Dannau. - Offa 38: 85-90.

Krzywinski, K. 1979. Preliminær undersøkelse av planterester i latrine. - Arkeo 1: 31-33.
- \& Fægri, K. 1979. Etnobotanisk bidrag til funksjonsanalyse. Eksempler fra middelalderunders 6 kelser i Bergen. - Arkeo 1: 33-39.

Kučan, D. 1979. Mittelalterliche Kulturpflanzen und Unkräuter aus ostfriesischen Kirschen. - Probleme Küstenforsch. südl. Nordseeg. 13: 23-38.

- 1981. Pflanzenreste aus dem Römerlager Oberaden. - Z. Archäol 15: $149-162$.

Lange, E. 1972. Ein Getreidefund von Hetzdorf aus der jüngeren vorrömischen Eisenzeit. - Z. Archäol. 6: 258-266.

1973. Unkräuter in frühgeschichtlichen Getreidefunden. - Ethnogr-Archäol. Z. 14: 193-221.

- 1975. Kultur- und Wildpflanzenfunde aus germanischen und slawischen Objekten. - Z. Archäol. 9: 301-306.

- 1976. Zur Entwicklung der natürlichen und anthropogene Vegetation in frühgeschichtlicher Zeit. - Feddes Repert. 87: 5-30.

- 1979. Zum Stand und einigen Ergebnissen paläo-ethnobotanischer Arbeiten in Brandenburg. - Gleditschia 7: 187-196.

Lange, J. 1975. Haveplanternes indførselshistorie i Danmark. I. Principper og metoder ved studiet af æeldre indførsler (indtil ca. 1700). - Fra Kvangård til Humlekule. Medd. Havebrugshistorisk Selskab 5: 17-28.

- 1979. Havebrug i Ribe indtil ca. 1800. - Fra Kvangård til Humlekule. Medd. Havebrugshistorisk Selskab 9: 7-44.

Lund, J. 1978. I en kælder ... - Skalk 1978 (1): 3-10.

Lynch, A. \& Paap, N. 1982. Untersuchungen an botanischen Funden aus der Lübecker Innenstadt. Ein Vorbericht. - Lübecker Schr. Archäol. Kulturgesch. 6: 339-360.

Mackeprang, M. B. 1934. Om et træskrin med amuletter og undergørende planter samt andet mærkeligt i en grav fra romersk jernalder. - Fra Nationalmuseets Arbejdsmark 1934: 42-50.

Madsen, V. 1902. Beskrivelse til geologisk kort over Danmark. Kortbladet Nyborg. - Danm. geol. Unders. I. række 9: 1-182.

- 1903. Om den glaciale, isdæmmede sø ved Stenstrup på Fyn samt om dannelsen af teglværksleret på Stenstrup-egnen. - Danm. geol. Unders. II. rakke 14: 1-88.

Magnusson, E. 1962. Lyby Mosse. En vegetations- och utvecklingshistorisk undersökning. - Sver. geol. Unders. Ser. C no 588, Årsb. 56 (4): $1-65$.

Mangerud, J., Andersen, S. T., Berglund, B. E. \& Donner, J. J. 1974. Quaternary stratigraphy of Norden, a proposal for terminology and classification. - Boreas 3: 109-128.

Menke, B. 1969. Vegetationskundliche und vegetationsgeschichtliche Untersuchungen an Strandwällen. - Mitt. Flor.-soz. Arb. Gemein. Heft 14: 95-120.

Mikkelsen, V. M. 1943. Bidrag til Lille Vildmoses stratigrafi og vegetationshistorie. - Meddr. Dansk geol. Foren. 10 (3): 329-364.

- 1949. Præst $\varnothing$ Fjord. The development of the post-glacial vegetation and a contribution to the history of the Baltic Sea. - Dansk Bot. Arkiv 13 (5): 1-171.

- 1952. Pollenanalytiske undersøgelser ved Bolle. Et bidrag til vegetationshistorien i subatlantisk tid. - In: Steensberg, A. (ed.), Bondehuse og vandmøller i Danmark gennem 2000 år. Nationalmuseets 3. Afd. Arkæologiske Landsbyundersøgelser I, pp. 109-132, 299-303.

- 1954. Studies on the sub-atlantic history of Bornholm's vegetation. - Danm. geol. Unders. II. række 80: 210-229.

- 1984. Landbrugets indflydelse på vegetationsudviklingen i Borup Ris. - Nytårs Gave til Borupris' Venner 1984-1985. Fuglebjerg.

Milthers, V. 1908. Beskrivelse til geologisk kort over Danmark. Kortbladene Faxe og Stevns Klint. - Danm. geol. Unders. I. række 11: 1-291.

- 1940. Beskrivelse til geologisk kort over Danmark. Kortbladet Vis senbjerg. - Danm. geol. Unders. I. række 19: 1-143.

Nilsson, T. 1935. Die pollenanalytische Zonengliderung der spätund post-glazialen Bildungen Schonens. - Geol. Fören. Stockholm Förhandl. 57: 385-562.

- 1948. A pollen-analytical investigation of Holmegårds Mose with considerations as to the age of the dwelling places of the Maglemo- 
sean period in Denmark and surrounding areas. - Meddr. dansk geol. Foren. 11: 201-217.

- 1958. Pollenanalytisk datering av stockbåt från Ruttenkalv, Färingtofta Socken. - Skånes hembygds förbunds Årsbok: 72-81.

- 1961. Ein neues Standardpollendiagramm aus Bjärsjöholmssjön in Schonen. - Lunds Universitets Årsskrift. N.F. Avd. 2, 56 (18): 1-34.

- 1964. Entwicklungsgeschichtliche Studien im Ageröds Mosse, Schonen. - Lunds Universitets Årsskrift. N.F. Avd. 2, 59 (8): 1-34.

- 1968. Pollenanalytische Datierung der Pfeilfunde aus Loshult im Nördlichsten Schonen. - Geol. Fören. Stockholm Förhandl. 90: 537-546.

Nordmann, V. 1915. On remains of reindeer and beaver from the commencement of the postglacial forest period in Denmark. Danm. geol. Unders. II. række 28: 1-24.

- 1922. Nye iagttagelser over den glaciale, isdæmmede sø ved Stenstrup pă Fyn. - Danm. geol. Unders. IV. rakke 1 (17): 1-25.

Olsen, O. 1976. Haveplanternes indførselshistorie i Danmark II. Principper og metoder ved studiet af senere indførsler (fra ca. 1700 og frem til vor tid). - Fra Kvangård til Humlekule. Medd. Havebrugshistorisk Selskab 6: 17-58.

- 1980. Planteindførsel til haven i Hellebæk 1891-1905-1956. Et usædvanligt initiativ af dr. F. Børgesen. - Fra Kvangård til Humlekule. Medd. Havebrugshistorisk Selskab 10: 27-60.

Overbeck, F. 1975. Botanisch-geologische Moorkunde. - Karl Wachholtz Verlag, Neumünster.

Pals, J. P. 1977. Seeds and fruits. - In: Bakker, J. A., Brandt, R. W., Geel, B. van, Jansma, M. J., Kuijper, W. J., Mensch, P. J. A. van, Pals, J. P. \& Ijzereef, G. F.(eds.), Hoogkarspel-Watertoren: towards a reconstruction of ecology and archaeology of an agrarian settlement of 1000 BC. - Ex Horreo (IPP 1951-1976), Cingvla IV, pp. 200-204.

-, Geel, B. van \& Delfos, A. 1980. Paleoecological studies in the Klokkeweel Bog near Hoogkarspel (prov. of Noord-Holland). Rev. Paleobot. Palynol. 30: 371-418.

Peglar, S. M. \& Wilson, D. G. 1978. The abandoned river channel. In: Drury, P. J. (ed.), Excavations at Little Waltham 1970-71. C. B. A. Research Report No. 26 and Chelmsford Excavation Committee, Report No. 1, pp. 146-148.

Piening, U. 1979. Neolithische Nutz- und Wildpflanzenreste aus Endersbach, Rems-Murr-Kreis, und Ilsfeld, Kreis Heilbronn. Fundb. Baden-Württemberg 4: 1-17.

Roesdahl, E. \& Nordquist, J. 1971. De døde fra Fyrkat. - Nationalmuseets Arbejdsmark 1971: 15-32.

Rostrup, E. 1877. En notits om plantevæxten i Danmark i "Broncealderen". - Aarb. N.O. 1877: 78-82.

- 1906. Frø resp. frugter og andre plantelevninger m.m. - In: Rosenkjær, H. N. (ed.), Fra det underjordiske København. Geologiske og historiske undersøgelser. Det Schønbergske Forlag, København, pp. 91-143.

Rowley-Conwy, P. 1979. Forkullet korn fra Lindebjerg. En boplads fra ældre bronzealder. - Kuml 1978: 159-171.

- (in print). A new sample of carbonised grain from Voldtofte. Kuml.

Rud, M. (ed.) 1979. Arkæologisk håndbog, 2. udgave. - Politikens Forlag, København.

Sandegren, R. 1931-32. Einige neue Funde von fossilen Najas flexilis in Schweden. - Abh. Naturwissenschaftlichen Verein zu Bremen Reihe B, band XXVIII: 235-245.

- 1941. Om den forna och nutida förekomsten av Najas flexilis i Sverige. - Bot. Notiser 1941: 59-64.

Sarauw, G. F. L. 1900. Dværghveden (Triticum compactum Host) og engelsk hvede (Triticum turgidum L.). Træk af to dyrkede hvedearters historie i Norden. - Bot. Tidsskr. 23: 83-99.

Scheer, K. 1955. Ergebnisse der Untersuchung der subfossilen Pflanzenreste der Grabung Tofting. - In: Bantelmann, A. (ed.), Tofting eine vorgeschichtliche Warft an der Eidermündung, Karl Wachholz Verlag, Neumünster, pp. 104-109.
Schiemann, E. 1957. Die Kulturpflanzenfunde in den spätkaiserzeitlichen Speichern von Kablow bei Königs-Wusterhausen, Mark. Berl. Blätter Vor- und Frühgesch. 6 (2): 100-124.

Schwaar, J. 1976. Paläogeobotanische Untersuchungen im Belmer Bruch bei Osnabrück. - Abh. Naturw. Verein Bremen 38 (2), Nr. 12: 207-257.

Stalling, H. 1983. Untersuchungen zur nacheiszeitlichen Vegetationsgeschichte des Meissners (Nordhessen). - Flora 174: 357-376.

Stockmarr, J. 1975. Biostratigraphic studies in Late Weichselian sediments near Böllingsö. - Danm. geol. Unders. Årbog 1974: 71-89.

Tallantire, P. H. 1979. Late Viking and Early Medieval plant material from Trondheim - a problem in interpretation. - Archaeo-Physika 8: 295-301.

Thomsen, T. 1929. Egekistefundet fra Egtved, fra den ældre bronzealder. - Nordiske Fortidsminder II: 165-201.

Tralau, H. 1974. Bibliography and index to palaeobotany and palynology. 1950-1970. - Hans Tralau, Stockholm.

Troels-Smith, J. 1943. Geologiske dateringer af bopladser i Aamosen. En foreløbig meddelelse. - In: Mathiassen, T. (ed.), Stenalderbopladser i Aamosen. Nordisk Forlag, København, pp. 147220 .

- 1953. Ertebøllekultur-bondekultur. Resultater af de sidste 10 års undersøgelser i Åmosen, Vestsjalland. - Årb. N.O. 1953: 1-62.

- 1955. Karakterisering af løse jordarter. - Danm. geol. Unders. IV rakke 3 (10): 1-73.

- 1957. Muldbjerg-bopladsen som den så ud for 4500 år siden. De første spor af agerbrug i Danmark. - Naturens Verden, Juli 1957: $1-30$.

- 1960. Ivy, mistletoe and elm. Climate indicators - fodder plants. A contribution to the interpretation of the pollen zone border VIIVIII. - Danm. geol. Unders. IV. rakke 4 (4): 1-32.

Tutin, T. G., Heywood, V. H., Burges, N. A., Moore, D. M., Valentine, D. H., Walters, S. M. \& Webb, D. A. (eds.) 1964-1980. Flora Europaea I-V. - University Press, Cambridge.

Usinger, H. 1978. Pollen- und grossrestanalytische Untersuchungen zur Frage des Bölling-Interstadials und der spätglazialen Baumbirken-Einwanderung in Schleswig-Holstein. - Schr. Naturw. Ver. Schlesw.-Holst. 48: 41-61.

- 1981. Pollen- und Grossrestanalysen an limnischen Spätglazial aus dem Scharnhagener Moor, Schleswig-Holstein. - Schr. Naturw. Ver. Schlesw.-Holst. 51: 85-105.

Wasylikowa, K. 1978. Plant remains from Early and Late medieval Time found on the Wawel Hill in Cracow. - Acta Palaeobotanica 19 (2): 115-198.

Westhusen, F. 1958. Die Kultur- und Wildfruchtfunde der Ausgrabungen in der Hamburger Altstadt 1948-1958. - Hammaburg 6: 195-198.

Wiermann, R. von 1962. Botanisch-moorkundliche Untersuchungen in Nordfriesland. - Meyniana 12: 97-146.

Wieserowa, A. 1979. Plant remains from the Early and Late Middle Ages found in the settlement layers of the main market square in Cracow. - Acta Palaeobotanica 20 (2): 137-212.

Willcox, G. H. 1977. Exotic plants from Roman waterlogged sites in London. - J. Archaeol. Sci. 4: 269-282.

Willerding, U. 1970. Vor- und frühgeschichtliche Kulturpflanzenfunde in Mitteleuropa. - Neue Ausgr. u. Forsch. in Niedersachsen 5: $287-375$.

- 1973. Frühmittelalterliche Pflanzenreste aus Braunschweig. Nachricht. Niedersachs. Urgesch. 42: 358-360.

- 1978a. Bibliographie zur Paläo-Ethnobotanik des Mittelalters in Mitteleuropa 1945-1977 (Teil 1). - ZAMA 6: 173-223.

- 1978b. Paläo-ethnobotanische Befunde an mittelalterlichen Pflanzenresten aus Süd-Niedersachsen, Nord-Hessen und dem östlichen Westfalen. - Ber. Deutsch. Bot. Ges. 91: 129-160.

- 1979a. Bibliographie zur Paläo-Ethnobotanik des Mittelalters in Mitteleuropa 1945-1977 (Teil 2). - ZAMA 7: 207-225.

- 1979b. Zum Ackerbau in der jüngeren vorrömischen Eisenzeit. Archaeo-Physika 8: 309-330. 
Willerding, U. 1979c. Vegetation und Ackerbau im Hannoverschen Wendland während des Mittelalters. - In: Rapport du III ${ }^{e}$ Congrès International d'Archéologie Slave, Bratislava 7-14 septembre 1975, Tome 1, Bratislava, pp. 905-915.

Wilson, D. G. 1968. Plant remains from a Roman well at Bunny, Nottinghamshire. - Transactions of the Thoroton Society of Nottinghamshire 72: 42-50.

- 1975. Plant remains from the Graveney. Boat and the carly history of Humulus lupulus L. in W. Europe. - New Phytol. 75: 627-648.

- 1978. Iron Age and Roman plant remains. - In: Drury, P. J. (ed.), Excavations at Little Waltham 1970-71. C. B. A. Research Report No. 26 and Chelmsford Excavation Committee, Report No. 1, pp. $142-145$.

- 1979. Horse dung from Roman Lancaster: A botanical report. Archaeo-Physika 8: 331-350.

Wittmack, L. \& Buchwald, J. 1902. Pflanzenreste aus der Hünenburg bei Rinteln a.d. Weser und eine verbesserte Methode zur Herstellung von Schnitten durch verkohlte Hölzer. - Ber. Deutsch. Bot. Ges. 20: 21-31.
Zeist, W. van 1968. Prehistoric and early historic food plants in the Netherlands. - Palaeohistoria 14: 41-173.

- 1974. Palaeobotanical studies of settlement sites in the coastal area of the Netherlands. - Palaeohistoria 16: 223-371.

- 1981. Plant remains from Iron Age Noordbarge, Province of Drenthe, The Netherlands. - Palaeohistoria 23: 169-193.

- \& Palfenier-Vegter, R. M. 1979. Agriculture in Medieval Gasselte. - Palacohistoria 21: 267-299.

- \& Palfenier-Vegter, R. M. 1981. Seeds and fruits from the Swifterbant S3 site. - Palaeohistoria 23: 105-168.

Ødum, H. 1920. Et elsdyrfund fra Tåderup på Falster. - Danm. geol. Unders. IV. række 1 (11): 1-16.

Ødum, S. 1965. Germination of ancient seeds. Floristical observations and experiments with archaeologically dated soil samples. Dansk Bot. Ark. 24 (2): 1-70.

- 1978. Dormant seeds in Danish ruderal soils. An experimental study of relations between seed bank and pioneer flora. - The Royal Vet. \& Agric. Univ. Hørsholm Arboretum, Denmark. 


\section{Table 1.}

References to publications containing information on macrofossils, listed according to site number (cf. Fig. 2), furnished with information on name of site, dating methods, periods, and medium examined for macrofossils.

\section{Abbreviations:}

$\begin{array}{lll}\text { Dating } & \text { Arch.: } & \text { Archaeological dating } \\ & \text { C-14: } & \text { Carbon-14 dating } \\ \text { Dendro.: } & \text { Dendrochronological dating } \\ \text { Mac.: } & \text { Macrofossil dating } \\ \text { Pol.: } & \text { Palynological dating } \\ \text { u: } & \text { Dating to the period uncertain } \\ -: & \text { No information } \\ \text { Period } & \text { I-IX: } & \text { Pollen assemblage zones } \\ & \text { B.C.: } & \text { Before the birth of Christ } \\ \text { A.D.: } & \text { After the birth of Christ } \\ \text { B.P.: } & \text { C-14 year before 1950 A.D. } \\ \text { NA: } & \text { Neolithic Age (3800-1801 B.C.) } \\ \text { ENA: } & \text { Early Neolithic Age* } \\ \text { MNA: } & \text { Middle Neolithic Age* } \\ \text { LNA: } & \text { Late Neolithic Age* } \\ \text { BA: } & \text { Bronze Age (1800-501 B.C.) }\end{array}$

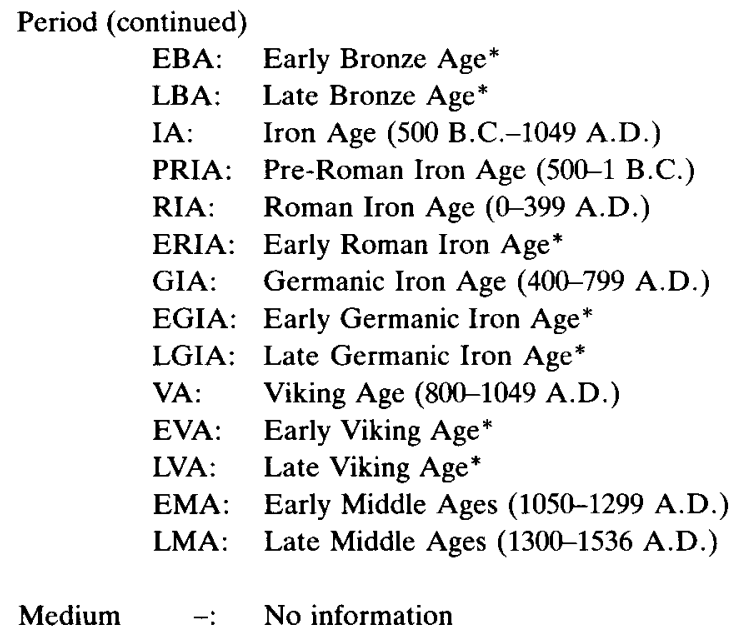

${ }^{*}$ The exact subdivision of Neolithic, Bronze Age, Roman Iron Age, Germanic Iron Age, and Viking Age is not specified in most publications.

\begin{tabular}{|c|c|c|c|c|c|}
\hline $\begin{array}{l}\text { Site } \\
\text { number }\end{array}$ & Site & $\begin{array}{l}\text { Author, year of publication, } \\
\text { page(s) }\end{array}$ & Dating & Period & Medium \\
\hline 101 & Bolle & Mikkelsen (1952): 114 & Pol. & Abt. 1 A.D. & Gyttja, peat \\
\hline 102 & Overbygård & Lund (1978): 8 & Arch. & PRIA & Storage \\
\hline 103 & Aggersborg & Jessen (1954): 127-133 & Arch. & VA & Pit \\
\hline 104 & Sejerslev Kær & Jessen (1929): 16-17 & Pol. & $\mathrm{I}-\mathrm{V}$ & Gyttja, lime, peat \\
\hline 105 & Vestervig & Ødum (1965): 52-53 & Arch. & Abt. 200 A.D. & Germination \\
\hline 106 & Nørhå & Hjelmqvist (1975a): 209-211 & Arch. & RIA & Imprint \\
\hline 107 & Ginnerup & Jessen (1933): 258-284 & Arch. & RIA & Storage \\
\hline 108 & $\emptyset$ sterbølle & Hatt (1938): $217-250$ & Arch. & RIA & Imprint, storage \\
\hline 109 & Borremose & Andersen (1975): 112 & Arch. & PRIA & Gyttja \\
\hline 110 & Fyrkat & Helbæk (1974): 6-36 & Arch. & VA & Grave, storage \\
\hline 111 & Gørding Hede & Helbæk (1951): 68 & Arch. & PRIA & Storage \\
\hline 112 & Troldtoft & Hatt (1935): 48 & Arch. & PRIA & Burnt house \\
\hline 113 & Bøllings $\emptyset$ & Brandt (1954): 157 & Pol. & I-III & $\begin{array}{l}\text { Late-glacial } \\
\text { sediments }\end{array}$ \\
\hline 114 & Tollund & Helbæk (1950): 326 & Arch. & PRIA & Stomach \\
\hline & & & \multicolumn{2}{|c|}{$\begin{array}{l}\text { Later C-14 dated to } 2160 \pm 40 \\
\text { B.P. (Fischer 1980) }\end{array}$} & \\
\hline 115 & $\begin{array}{l}\text { Nebelgårds } \\
\text { Mose, } \\
\text { Grauballe }\end{array}$ & Helbæk (1958a): 84-85 & $\begin{array}{l}\text { C-14 } \\
\text { Later C } \\
\text { B.C. (F }\end{array}$ & $\begin{array}{l}\text { 3rd-5th cent. } \\
\text { A.D. } \\
\text { d to } 80 \pm 55 \\
980)\end{array}$ & Stomach \\
\hline 116 & Vadgård & Jørgensen (1979): 137 & $\mathrm{C}-14$ & 1550 B.C. & House \\
\hline 117 & $\begin{array}{l}\text { Bækmoien, } \\
\text { Frederikshavn }\end{array}$ & Hatt (1937): 30-33 & Arch. & RIA & Imprint \\
\hline 118 & Rakkeby & Hatt (1937): $30-33$ & Arch. & RIA & Imprint \\
\hline 119 & Albæk Hede & Hatt (1937): 31 & Arch. & PRIA & Imprint \\
\hline 120 & Vestervig & Hatt (1937): 33 & Arch. & RIA & Imprint \\
\hline 121 & Solbjerg & Hatt (1937): 28 & Arch. & PRIA & Storage \\
\hline 122 & Freds $\varnothing$ & Hatt (1937): 31 & Arch. & GIA & Imprint \\
\hline 123 & Vindblæs Hede & Hatt (1937): 31 & Arch. & PRIA & Imprint \\
\hline 124 & Engelstrup & Hatt (1937): 33 & Arch. & RIA & Imprint \\
\hline 125 & Malle & Hatt (1937): $30-35$ & Arch. & RIA & Imprint \\
\hline 126 & Vive & Hatt (1937): $30-31$ & Arch. & RIA & Imprint \\
\hline
\end{tabular}


Table 1 continued

\begin{tabular}{|c|c|c|c|c|c|}
\hline $\begin{array}{l}\text { Site } \\
\text { number }\end{array}$ & Site & $\begin{array}{l}\text { Author, year of publication, } \\
\text { page(s) }\end{array}$ & Dating & Period & Medium \\
\hline 127 & Borremose & Brandt (1951): 348 & \multicolumn{2}{|c|}{$\begin{array}{l}\text { Later C-14 dated to } 650 \\
\pm 80 \text { B.C. (Fischer 1980) }\end{array}$} & Stomach \\
\hline 128 & Lille Vildmose & Mikkelsen (1943): 345-346 & Arch. & Abt. 100 A.D. & Sand \\
\hline 129 & Signalbakken & Jessen \& Lind (1922-23): 17 & Arch. & NA & Imprint \\
\hline 130 & Fars $\emptyset$ & Jessen \& Lind (1922-23): 18 & Arch. & EGIA & Imprint \\
\hline 131 & Lundtoft & Jessen \& Lind (1922-23): 18 & Arch. & RIA & Imprint \\
\hline 132 & Ørumå, Grenå & Jessen \& Lind (1922-23): 17 & Arch. & NA & Imprint \\
\hline 133 & Thorsager & Jessen \& Lind (1922-23): 18 & Arch. & EGIA & Imprint \\
\hline 134 & Rostved & Jessen \& Lind (1922-23): 18 & Arch. & RIA & Imprint \\
\hline 135 & Hovlbjerg & Jessen \& Lind (1922-23): 21 & Arch. & EGIA & Soil \\
\hline 136 & Tungelund, Aidt & Jessen \& Lind (1922-23): 18 & Arch. & RIA & Imprint \\
\hline 137 & Freds $\varnothing$ & Hatt (1930): 87 & Arch. & PRIA-RIA & Burnt house \\
\hline 138 & Fjerritslev & Iversen (1934): 7-10 & Pol. & III-IV & Clay, gyttja \\
\hline 139 & Fjand & Helbæk (1954): $257-259$ & Arch. & ERIA & Storage \\
\hline 140 & $\begin{array}{l}\text { Mariesminde, } \\
\text { Vestervig }\end{array}$ & Hatt (1960): 80-81 & Arch. & RIA & $\begin{array}{l}\text { Burnt house, } \\
\text { imprint }\end{array}$ \\
\hline 141 & Kraghede & Brøndsted (1940): 81 & Arch. & PRIA & Imprint \\
\hline 142 & Kettrup & Brøndsted (1938): 359 & Arch. & MNA & Imprint \\
\hline 143 & Allestrup & Brøndsted (1938): 355 & Arch. & NA & Imprint \\
\hline 144 & Næsborg & Brøndsted (1938): 349 & Arch. & NA & Imprint \\
\hline 145 & Stenvad & Brøndsted (1938): 342 & Arch. & NA & Imprint \\
\hline 146 & Bækmoien & Hatt (1959): 209-213 & Arch. & ERIA & Imprint \\
\hline 147 & Sjørring & $\emptyset$ dum (1965): 52-53 & Arch. & Abt. 1300 A.D. & Germination \\
\hline 148 & Skørbæk Hede & Hatt (1938): 166 & Arch. & PRIA & Imprint \\
\hline 149 & Emmedsbo & Brønsted (1938): 341 & Arch. & NA & Imprint \\
\hline 150 & Skørbæk & Brønsted (1938): 341 & Arch. & NA & Imprint \\
\hline 151 & Alrum & Helbæk (1954): 257-259 & Arch. & ERIA & Storage \\
\hline 152 & $\begin{array}{l}\text { Lønstrup Klint: } \\
\text { Martørv Bakker }\end{array}$ & Hartz (1902): 52 & Mac. & III u & Sand \\
\hline 153 & $\begin{array}{l}\text { Lønstrup Klint: } \\
\text { Lyngby }\end{array}$ & Hartz (1902): 53 & Mac. & III u & - \\
\hline 154 & Fyrkat & Roesdahl \& Nordquist (1971): 30 & Arch. & VA & Grave \\
\hline 155 & Bøllingsø & Stockmarr (1975): 83 & Pol., C-14 & I-III & $\begin{array}{l}\text { Gyttja, peat, } \\
\text { sand }\end{array}$ \\
\hline 156 & Ulfborg & Jonassen (1954): 137 & Pol. & IX & Gyttja \\
\hline 157 & Krags $\varnothing$, Karup & Jonassen (1936): 190 & Pol. & IV-IX & Gyttja \\
\hline 158 & Herning Teglværk & Jessen (1939a): 79 & Mac. & IIIu & Gyttja, sand \\
\hline 159 & Hors $\emptyset$ & Jessen (1927): 135 & Pol. & VII & Gyttja \\
\hline 160 & $\begin{array}{l}\text { Nebelgårds } \\
\text { Mose }\end{array}$ & Jørgensen (1956): 116 & Pol., C-14 & $\begin{array}{l}\text { III, Va Zone } \\
\text { border IV/Va, } \\
\text { dated to } 8350 \\
\pm 350 \text { B.C. }\end{array}$ & Gyttja, peat \\
\hline 161 & Solsø, Videbæk & Jessen \& Milthers (1928): 117-118 & Mac. & III u & - \\
\hline 162 & Skallesøgård & Jonassen (1950): 86 & Pol. & VIII-IX & Peat \\
\hline 163 & Hjerl & Jonassen (1950): 87 & Pol. & VIII & Peat \\
\hline 164 & Fly, Skive & Jonassen (1950): 89 & Pol. & VII-IX & Gyttja, peat \\
\hline 165 & Høkjær & Jonassen (1950): 85 & Pol. & III-VII & Peat \\
\hline 166 & Birks $\varnothing$ & Jonassen (1950): 83 & Pol. & V-VI & Peat \\
\hline 167 & Over Feldborg & Jonassen (1950): 90 & Pol. & IX & Peat \\
\hline 168 & Krags $\varnothing$ & Jonassen (1950): 91 & Pol. & V-IX & Gyttja \\
\hline 169 & Damholt & Jonassen (1950): 95 & Pol. & V-VI & Peat \\
\hline 170 & Ravnholt & Jonassen (1950): 96-97 & Pol. & V-VII, IX & Gyttja, peat \\
\hline 171 & Asmild & $\emptyset$ dum (1965): $52-53$ & Arch. & Abt. 1300 A.D. & Germination \\
\hline 172 & Viborg & Jensen, under preparation & Arch. & VA-EMA & Soil \\
\hline 173 & $\emptyset$ sterbølle & Helbæk (1959): 115 & Arch. & 1st cent. A.D. & Imprint, storage \\
\hline 174 & Alrum & Helbæk (1959): 115 & Arch. & 1st cent. A.D. & Storage \\
\hline 175 & Brøndum Mose & Jessen (1934): 191 & Pol. & IV-VII & Peat \\
\hline 176 & Sterbygård Mose & Jessen (1934): 196 & Pol. & VIII-IX & Peat \\
\hline 177 & Fussing $\varnothing$ Mose & Jessen (1934): 205 & Pol. & VI-VII & Peat \\
\hline 178 & Lodbjerg & Jensen \& Liversage (unpubl.) & C-14 & 355-240 B.C. & Sandy peat \\
\hline
\end{tabular}


Table 1 continued

\begin{tabular}{|c|c|c|c|c|c|}
\hline $\begin{array}{l}\text { Site } \\
\text { number }\end{array}$ & Site & $\begin{array}{l}\text { Author, year of publication, } \\
\text { page(s) }\end{array}$ & Dating & Period & Medium \\
\hline 179 & Dyrholmen & Andersen et al. (1983): 96 & C-14 & c. 4000 B.C. & Sand \\
\hline 180 & Løvenholm & Andersen (1984): 84, 87 & C-14, Pol. & $\begin{array}{l}5400 \pm 120 \text { B.P. } \\
\text { VIII, IX }\end{array}$ & Peat, sandy gyttja \\
\hline 201 & $\begin{array}{l}\emptyset \text { ster Lem } \\
\text { Hede }\end{array}$ & Hatt (1949): 97 & Arch. & PRIA & Imprint \\
\hline 202 & $\begin{array}{l}\text { Århus } \\
\text { Søndervold }\end{array}$ & Fredskild (1971): 314-317 & Arch. & LVA & Burnt house, pit \\
\hline 203 & Birknæs & Helbæk (1952 a): 99 & \multicolumn{2}{|c|}{$\begin{array}{l}\text { Later C-14 dated to } 1985 \text { B.C. } \\
\text { (Jørgensen } 1979, \text { p. } 140 \text { ) }\end{array}$} & Pit, storage \\
\hline 204 & $\emptyset_{\text {stbirk }}$ & Helbæk (1952 a): 105 & \multicolumn{2}{|c|}{$\begin{array}{l}\text { Later arch. dated to } 2400-2250 \\
\text { B.C. (Jørgensen } 1979, \text { p. } 140)\end{array}$} & Pit, storage \\
\hline 205 & Oksbøl & Helbæk (1958 b): 156 & Arch. & 6th cent. A.D. & Burnt house \\
\hline 206 & Vorbasse & Jørgensen (1977 a): 234 & C-14 & 1810 B.C. & Burnt house \\
\hline 207 & Jels & Iversen (1939): 19 & Arch. & BA & Soil \\
\hline 208 & Bunds $\emptyset$ & Jessen $(1939$ b): 65 & Arch. & MNA & $\begin{array}{l}\text { Burnt house, } \\
\text { imprint }\end{array}$ \\
\hline 209 & Hjortspring & Jessen (1937): 26-27 & Pol. & III-IV, IX & $\begin{array}{l}\text { Clay, gyttja, } \\
\text { peat }\end{array}$ \\
\hline 210 & Grønbjerg & Hatt (1937): 33 & Arch. & RIA & Imprint \\
\hline 211 & Voldsted & Hatt (1937): 31 & Arch. & PRIA & Imprint \\
\hline 212 & $\begin{array}{l}\text { Majbølgård, } \\
\text { Als }\end{array}$ & Hatt (1937): 35 & Arch. & RIA & Imprint \\
\hline 213 & Bunds $\varnothing$ & Jessen (1938 a): 131 & Pol. & V-VIII & Gyttja, sand \\
\hline 214 & Birknæs & Jessen \& Lind (1922-23): 20 & $\begin{array}{l}\text { Arch. } \\
\text { Later C-14 } \\
\text { (Jørgensen }\end{array}$ & $\begin{array}{l}\text { BA } \\
\text { ed to } 1985 \text { B. C. } \\
9, \text { p. 140) }\end{array}$ & Pit, storage \\
\hline 215 & Nortvig & Jessen \& Lind (1922-23): 18 & Arch. & RIA & Imprint \\
\hline 216 & Møllerup & Jessen \& Lind (1922-23): 18 & Arch. & RIA & Imprint \\
\hline 217 & Boringholm & Jessen (1919): 52 & Arch. & $\begin{array}{l}\text { Middle Ages, } \\
\text { most likely } \\
\text { before } 1406 \\
\text { A.D. }\end{array}$ & Gyttja, peat \\
\hline 218 & Boringholm & Jessen \& Lind (1922-23): 24-25 & Arch. & $\begin{array}{l}\text { Middle Ages, } \\
\text { most likely } \\
\text { before } 1406 \\
\text { A.D. }\end{array}$ & Gyttja, peat \\
\hline 219 & Jels & Broholm (1938): 5-6 & Arch. & EBA & Soil \\
\hline 220 & Egtved & Thomsen (1929): $180-200$ & Arch. & EBA & Grave, soil \\
\hline 221 & Bundsø & Jessen $(1938 b): 58-60$ & Pol. & V-VIII & Gyttja, sand \\
\hline 222 & Eltang Vig & Jessen \& Lind (1922-23): 18 & Arch. & EGIA & Imprint \\
\hline 223 & Brabrand & Hatt (1937): 24 & Arch. & NA & Imprint \\
\hline 224 & Lindskov & Hatt (1937): 24 & Arch. & NA & Imprint \\
\hline 225 & Tyregodlund & Brøndsted (1938): 359 & Arch. & MNA & Imprint \\
\hline 226 & Asbo & Brøndsted (1938): 355 & Arch. & NA & Imprint \\
\hline 227 & Bunds $\emptyset$ & Brøndsted (1938): 358 & Arch. & MNA & Imprint \\
\hline 228 & Oksbøl & Hatt (1948): 44-59 & Arch. & GIA & $\begin{array}{l}\text { Burnt house, } \\
\text { imprint }\end{array}$ \\
\hline 229 & Arnum & Iversen (1939): 18 & Arch. & $\mathrm{BA}$ & Soil \\
\hline 230 & Skrydstrup & Iversen (1939): 19-20 & Arch. & BA & Soil \\
\hline 231 & Gårdslev & Brøndsted (1938): 359 & Arch. & MNA & Imprint \\
\hline 232 & Klelund & Brøndsted (1938): 359 & Arch. & MNA & Imprint \\
\hline 233 & Lindskov & Brøndsted (1938): 358 & Arch. & MNA & Imprint \\
\hline 234 & Lykkeby & Brøndsted (1938): 358 & Arch. & MNA & Imprint \\
\hline 235 & Esbjerg & Hartz (1902): 58-59 & Mac. & IV-VII u & Peat \\
\hline 236 & Hegum & Brøndsted (1938): 341-342 & Arch. & NA & Imprint \\
\hline 237 & Vonsild & Jessen, A. (1935): 73 & Mac. & II-III & Clay, gyttja \\
\hline 238 & $\begin{array}{l}\text { Lundbæk } \\
\text { Mose }\end{array}$ & Hartz (1902): 61-62 & Mac. & IV-V u & Gyttja \\
\hline 239 & $\begin{array}{l}\text { Nørre } \\
\text { Kollemorten }\end{array}$ & Jessen $(1939$ c): 138 & Pol. & IV & Peat \\
\hline
\end{tabular}


Table 1 continued

\begin{tabular}{|c|c|c|c|c|c|}
\hline $\begin{array}{l}\text { Site } \\
\text { number }\end{array}$ & Site & $\begin{array}{l}\text { Author, year of publication, } \\
\text { page(s) }\end{array}$ & Dating & Period & Medium \\
\hline 240 & $\begin{array}{l}\text { Cistercian } \\
\text { Monastery, } \emptyset_{m}\end{array}$ & Jensen, under preparation & $\begin{array}{l}\text { Arch. Nu- } \\
\text { mismatic }\end{array}$ & LMA & $\begin{array}{l}\text { House remains, } \\
\text { sand }\end{array}$ \\
\hline 241 & $\begin{array}{l}\text { Borchs Gard, } \\
\text { Kolding }\end{array}$ & Jensen, under preparation & $\begin{array}{l}\text { Arch. Nu- } \\
\text { mismatic }\end{array}$ & LMA & $\begin{array}{l}\text { Humus, manure, } \\
\text { soil }\end{array}$ \\
\hline 242 & $\begin{array}{l}\text { Kunstmuseet, } \\
\text { Ribe }\end{array}$ & Jensen, under preparation & $\begin{array}{l}\text { Arch. Nu- } \\
\text { mismatic }\end{array}$ & 8th cent. A.D. & Manure \\
\hline 243 & $\begin{array}{l}\text { Dommerhaven, } \\
\text { Ribe }\end{array}$ & Jensen, under preparation & $\begin{array}{l}\text { Arch. Nu- } \\
\text { mismatic }\end{array}$ & 8th cent. A.D. & Manure \\
\hline 244 & Tvedgade, Ribe & Jensen, under preparation & Arch. & 8th cent. A.D. & Manure \\
\hline 245 & $\begin{array}{l}\text { Sønderports- } \\
\text { gade, Ribe }\end{array}$ & Jensen, under preparation & Arch. & EMA-LMA & $\begin{array}{l}\text { Peat with } \\
\text { culture remains, } \\
\text { sand }\end{array}$ \\
\hline 301 & Archsum, Sylt & Kroll (1975): Table 15 & Arch. & $\mathrm{BA}-\mathrm{VA}$ & Soil, storage \\
\hline 302 & Amrum & Feindt (1975): 42-47 & Arch. & 50B.C. -50 A.D. & Storage \\
\hline 303 & Westerohrstedt & Hinz (1954): 87 & Arch. & RIA & House \\
\hline 304 & Haithabu & Behre (1969): 23-42 & Arch. & VA & Pit, soil, storage \\
\hline 305 & Alt-Schleswig & Behre (1978): 169 & Arch. & EMA-LMA & Pit, soil \\
\hline 306 & Tofting & Scheer (1955): 104-106 & Arch. & RIA-VA & Soil \\
\hline 307 & Elisenhof & Behre (1976): 86-133 & Arch. & $\begin{array}{l}\text { 8th-10th cent. } \\
\text { A.D. }\end{array}$ & Soil \\
\hline 308 & Archsum, Sylt & Kroll (1980): 378 & Arch. & 1 A.D. & $\begin{array}{l}\text { Burnt house, } \\
\text { storage }\end{array}$ \\
\hline 309 & Ülsby & Kroll (1980): 375 & Arch. & $\begin{array}{l}\text { PRIA }-1 \mathrm{st} \\
\text { cent. A.D. }\end{array}$ & Storage \\
\hline 310 & Haithabu & Behre (1978): 169 & Arch. & VA & Pit, soil \\
\hline 311 & Tofting & Behre (1976): 75-78 & Arch. & RIA & Soil \\
\hline 312 & Haithabu & Behre (1981): 26-29 & Arch. & VA & Pit, soil, storage \\
\hline 313 & Kubitzbergmoor & Usinger (1978): 53-54 & Pol. & I-II & Gyttja \\
\hline 314 & $\begin{array}{l}\text { Ravensberg- } \\
\text { moor }\end{array}$ & Usinger (1978): $52-53$ & Pol. & I-II & Gyttja \\
\hline 315 & Tholendorf & Menke (1969): 116 & $\begin{array}{l}\text { Arch., } \\
\text { Pol. }\end{array}$ & RIA & Burnt layer \\
\hline 316 & Westerohrstedt & Hinz (1951): 62 & Arch. & RIA & Burnt house \\
\hline 317 & Büdelsdorf & Kroll (1976): 62 & Arch. & ENA & Imprint \\
\hline $319^{*}$ & $\begin{array}{l}\text { Scharnhagener } \\
\text { Moor }\end{array}$ & Usinger (1981): 97 & Pol. & I-IV & Gyttja, silt \\
\hline 320 & Elisenhof & Behre (1975): 59-60 & Arch. & VA & Soil \\
\hline 321 & Gotteskoog - I & Wiermann (1962): 102 & Pol. & VI-VII & Clay, peat \\
\hline 322 & Ockholm - II & Wiermann (1962): 102 & Pol. & IV-VIII & Clay, gyttja, peat \\
\hline 323 & Ockholm - I & Wiermann (1962): 102 & Pol. & VI - IX & Gyttja, peat \\
\hline 324 & Altendeich - I & Wiermann (1962): 103 & Pol. & IX & Peat \\
\hline 325 & Wallsbüll - I & Wiermann (1962): 103 & Pol. & IV-IX & Peat \\
\hline 326 & Wobbenbüll - I & Wiermann (1962): 104 & Pol. & VII-IX & Peat \\
\hline 327 & Hattstedt - I & Wiermann (1962): 104 & Pol. & VIII-IX & Peat \\
\hline 328 & Bohmstedt - I & Wiermann (1962): 104 & Pol. & VIII-IX & Clay, peat \\
\hline 329 & Bohmstedt - II & Wiermann (1962): 105 & Pol. & VIII-IX & Clay, peat \\
\hline 330 & Ahrenshöft - I & Wiermann (1962): 105 & Pol. & III-IV & Peat, sand \\
\hline 331 & $\begin{array}{l}\text { Nordstrandisch- } \\
\text { moor }\end{array}$ & Wiermann (1962): 105-106 & Pol. & VIII-IX & Peat \\
\hline 332 & Nordstrand & Wiermann (1962): 106 & Pol. & IX & Clay, peat \\
\hline 333 & Haithabu & Behre (1981): 26-37 & Arch. & VA & Pit, soil \\
\hline 334 & Haithabu & Behre (1983): 135-182 & Arch. & VA & Pit, soil \\
\hline 401 & Lindebjerg & Rowley-Conwy (1979): 160 & Arch. & EBA & Pit, storage \\
\hline 402 & Ladby & Jessen (1954): 129-130 & Arch. & VA & Grave \\
\hline 403 & Voldtofte & Helbæk (1952 a): 105 & Arch. & LBA & Storage \\
\hline & & & \multicolumn{2}{|c|}{$\begin{array}{l}\text { Re-dated to approx. } 800-700 \\
\text { B.C. }\end{array}$} & \\
\hline 404 & Sarup & Andersen (1980): 72-95 & C-14 & $\begin{array}{l}2630 \pm 90 \text { B.C. } \\
2530 \pm 90 \text { B.C. } \\
2450 \pm 90 \text { B.C. } \\
2390 \pm 90 \text { B.C. }\end{array}$ & Pit, soil, storage \\
\hline
\end{tabular}


Table 1 continued

\begin{tabular}{|c|c|c|c|c|c|}
\hline $\begin{array}{l}\text { Site } \\
\text { number }\end{array}$ & Site & $\begin{array}{l}\text { Author, year of publication, } \\
\text { page(s) }\end{array}$ & Dating & Period & Medium \\
\hline 405 & Sarup & Jørgensen $(1977$ b): 58 & \multicolumn{2}{|c|}{$\begin{array}{l}\text { Acc. to Andersen, N. H. } \\
\text { (1980): } 68 \text { dated by C- } 14 \text { to } \\
2630 \pm 90 \text { B.C. }\end{array}$} & Storage \\
\hline 406 & Svendborg & Jørgensen (1980): 204-206 & Arch. & $\begin{array}{l}\text { Approx. } 1350- \\
1400 \text { A.D. }\end{array}$ & Latrine \\
\hline 407 & Flådet & Fredskild (1979): 18 & $\mathrm{C}-14$ & $\begin{array}{l}7660 \pm 100 \text { B.P. } \\
7970 \pm 130 \text { B.P } \\
9120 \pm 130 \text { B.P. }\end{array}$ & Gyttja, peat \\
\hline 408 & Stengade & Fredskild (1977): 25-26 & Arch. & 10th cent. A.D. & Grave \\
\hline 409 & Stengade & Hjelmqvist (1975 b): 218 & $\begin{array}{l}\text { Thermolu- } \\
\text { minescence }\end{array}$ & 3900-330 B.C. & Imprint \\
\hline 410 & $\begin{array}{l}\text { Gammelung } \\
\text { Mose }\end{array}$ & Jessen (1938 a): 127 & Pol. & II-VIII & Gyttja, sandy clay \\
\hline 411 & Voldtofte & Jessen \& Lind (1922-23): 18-19 & Arch. & LBA & Soil \\
\hline 412 & $\begin{array}{l}\text { Brænde } \\
\text { Lydinge }\end{array}$ & Mackeprang (1934): 48 & Arch. & RIA & Grave \\
\hline 413 & Borreby & Brøndsted (1938): 362 & Arch. & LNA & Imprint \\
\hline 414 & Lindeskov & Brøndsted (1938): 358 & Arch. & LNA & Imprint \\
\hline 415 & Ejby & Hartz (1902): 37 & Mac. & III u & Clay \\
\hline 416 & Stenstrup & Hartz (1902): $40-43$ & Mac. & I u-III u & Clay, gyttja \\
\hline 417 & Stenstrup & Madsen (1903): $25-27$ & Mac. & I u-III u & Clay, gyttja, sand \\
\hline 418 & Akkerup Mose & Degerbøl \& Iversen (1945): 37-41 & Pol. & II-IV u & Gyttja, peat \\
\hline 419 & Stenstrup & Nordmann (1922): 4-15 & Mac. & II-III & Clay, gyttja \\
\hline 420 & Flådet & Fredskild (1975): 152 & Pol. & V-VII & Gyttja, peat \\
\hline 421 & Lørup Hede & Jessen (1916): XVII & Mac. & IV u-VIII u & $\begin{array}{l}\text { Calcareous gyttja, } \\
\text { peat }\end{array}$ \\
\hline 422 & $\begin{array}{l}\text { Stevningen } \\
\text { Mose }\end{array}$ & Madsen (1902): 115-118 & Mac. & $\begin{array}{l}\text { The Oak Period } \\
\text { (VII u) }\end{array}$ & Gyttja, peat \\
\hline 423 & Skalbjerg & Milthers (1940): 100 & Mac. & III $\mathbf{u}$ & Clay \\
\hline 424 & Hindevad & Milthers (1940): 105 & Mac. & III $\mathbf{u}$ & Clay \\
\hline 425 & Voldtofte & Rowley-Conwy (in print) & Arch. & LBA & Soil \\
\hline 426 & $\begin{array}{l}\text { Kjellerup, } \\
\text { Ringe }\end{array}$ & Nordmann (1915): 10-11 & Mac. & III $\mathbf{u}$ & Clay \\
\hline 427 & $\begin{array}{l}\text { Black Friars' } \\
\text { Monastery, } \\
\text { Odense }\end{array}$ & Jensen, under preparation & Arch. & VA-LMA & $\begin{array}{l}\text { Clay, house } \\
\text { remains, peat, } \\
\text { sand }\end{array}$ \\
\hline 428 & $\begin{array}{l}\text { Foldagers Gård, } \\
\text { Svendborg }\end{array}$ & Jensen $(1979$ a): 19-23, 59-67 & $\begin{array}{l}\text { Arch., } \\
\text { C-14, } \\
\text { Dendro. }\end{array}$ & $\begin{array}{l}\text { EMA-LMA } \\
1100 \pm 50 \text { A.D. } \\
1160 \pm 50 \text { A.D. } \\
1159 \text { A.D. } 1228 \\
\text { A.D. }\end{array}$ & $\begin{array}{l}\text { Humous culture } \\
\text { layer }\end{array}$ \\
\hline 429 & $\begin{array}{l}\text { Korsgade } 4 \\
\text { Svendborg }\end{array}$ & Jensen (1979 a): 29-32 & Arch. & EMA-LMA & $\begin{array}{l}\text { Humous-sandy } \\
\text { culture layer }\end{array}$ \\
\hline 430 & $\begin{array}{l}\text { Krøyer's Have, } \\
\text { Svendborg }\end{array}$ & Jensen (1979 a): $35-38$ & $\begin{array}{l}\text { Arch., } \\
\text { C-14 }\end{array}$ & $\begin{array}{l}\text { EMA-LMA } \\
1210 \pm 50 \text { A.D. } \\
1350 \pm 50 \text { A.D. }\end{array}$ & Clay, gyttja, sand \\
\hline 431 & $\begin{array}{l}\text { The Franciscan } \\
\text { Monastery, } \\
\text { Svendborg }\end{array}$ & Jensen (1979 a): 42-44 & Arch. & EMA & $\begin{array}{l}\text { Peat with culture } \\
\text { remains }\end{array}$ \\
\hline 501 & Maglemose & Jessen (1920): 111 & Pol. & VI u-IX & Gyttja, peat \\
\hline 502 & Lille Gribsø & Jessen (1920): 122 & Pol. & II-IX & Clay, gyttja, peat \\
\hline 503 & Kvistgård & Hartz (1902): 32 & Mac. & III $u$ & Clay \\
\hline 504 & Ebelholt & Ødum (1965): 53 & Arch. & Abt. 1500 A.D. & Germination \\
\hline 505 & $\begin{array}{l}\text { Hjortegårdene, } \\
\text { Dråby }\end{array}$ & Brøndsted (1938): 341-342 & Arch. & NA & Imprint \\
\hline 506 & Nivå & Jessen (1920): 156-157 & Pol., Mac. & III u, V u & Clay-gyttja \\
\hline 507 & Rungsted & Jessen (1923): 7 & Mac. & III $\mathrm{u}$ & Clay \\
\hline 508 & Warmings Mose & Jessen (1920): $69-70$ & Pol. & II-IX & Clay, gyttja \\
\hline 509 & Frihedens Mose & Jessen (1920): $82-83$ & Mac. & II-III & Clay, gyttja \\
\hline
\end{tabular}


Table 1 continued

\begin{tabular}{|c|c|c|c|c|c|}
\hline $\begin{array}{l}\text { Site } \\
\text { number }\end{array}$ & Site & $\begin{array}{l}\text { Author, year of publication, } \\
\text { page(s) }\end{array}$ & Dating & Period & Medium \\
\hline 510 & Allerød & Hartz (1902): 19-20 & Mac. & I-III & Clay, gyttja \\
\hline 511 & Allerød & Hartz \& Milthers (1901): 52-53 & Mac. & I-III & Clay, gyttja \\
\hline 512 & Vig & Hartz \& Winge (1906): $229-230$ & Mac. & III u-IV u & Gyttja, peat \\
\hline 513 & Sækkedam & Jessen $(1920): 19-27$ & Pol. & I-IX & $\begin{array}{l}\text { Clay, gyttja, } \\
\text { peat }\end{array}$ \\
\hline 514 & Birkerød & Hatt (1937): 37 & Arch. & NA & Imprint \\
\hline 515 & Birkerød & Brøndsted (1938): 139-141 & Arch. & NA & Imprint \\
\hline 516 & Kromose & Jessen $(1920): 88$ & Mac. & III $u$ & Clay \\
\hline 517 & Hestetang Huse & $\varnothing$ dum (1965): 53 & Arch. & Abt. 1500 A.D. & Germination \\
\hline 518 & $\begin{array}{l}\text { Vejlekro, } \\
\text { Fårevejle }\end{array}$ & Brøndsted (1938): 349 & Arch. & NA & Imprint \\
\hline 519 & $\begin{array}{l}\text { Trammose, } \\
\text { Udby }\end{array}$ & Brøndsted (1938): 342 & Arch. & NA & Imprint \\
\hline 520 & $\begin{array}{l}\text { Københavns } \\
\text { Frihavn }\end{array}$ & Jessen (1920): 143-144 & Mac. & VII u & Peat \\
\hline 521 & København & Rostrup (1906): 41-143 & $\begin{array}{l}\text { (cf. Jessen } \\
\text { \& Lind } \\
\text { 1922-23: } \\
21-22 \text { ) }\end{array}$ & EMA-LMA & $\begin{array}{l}\text { House, soil, } \\
\text { storage }\end{array}$ \\
\hline 522 & København & $\begin{array}{l}\text { Jessen \& Lind (1922-23): } \\
24-25\end{array}$ & Arch. & EMA-LMA & Mud, peat, soil \\
\hline 523 & $\begin{array}{l}\text { Faurbo, } \\
\text { Jyderup }\end{array}$ & Jessen (1924 a): 5-8 & Pol. & II-III & Clay, gyttja \\
\hline 524 & Himmelev & Jessen \& Lind (1922-23): 17 & Arch. & NA & Imprint \\
\hline 525 & Himmelev & Brøndsted (1938): 358 & Arch. & MNA & Imprint \\
\hline 526 & Årby & Brøndsted (1938): 358 & Arch. & MNA & Imprint \\
\hline 527 & Kornerup & Brøndsted (1938): 358 & Arch. & MNA & Imprint \\
\hline 528 & Saltholm Flak & Jessen (1920): 150 & Mac. & VI-VII u & Gyttja \\
\hline 529 & $\begin{array}{l}\text { Kildegård, } \\
\text { Åmosen }\end{array}$ & Jørgensen (1963): 27-29 & Pol. & V-VI & Gyttja, peat \\
\hline 530 & $\emptyset$ gårde & Troels-Smith (1943): 151 & $\begin{array}{l}\text { Arch., } \\
\text { Pol. }\end{array}$ & V-VI & Gyttja \\
\hline 531 & Muldbjerg & Troels-Smith (1957): 18-22 & $\begin{array}{l}\text { Arch., } \\
\text { C-14, Pol. }\end{array}$ & $2630 \pm 80$ B.C. & Gyttja, peat \\
\hline 532 & Åmosen & Troels-Smith (1960): 11 & $\begin{array}{l}\text { Arch., } \\
\text { Pol. }\end{array}$ & NA & Imprint \\
\hline 533 & Åmosen & Troels-Smith (1953): 23 & $\begin{array}{l}\text { Arch., } \\
\text { Pol. }\end{array}$ & NA & Imprint \\
\hline 534 & Magle $\emptyset$ & Troels-Smith (1943): 153-154 & $\begin{array}{l}\text { Arch., } \\
\text { Pol. }\end{array}$ & V-VI & Gyttja, peat \\
\hline 535 & Verup, Åmosen & Jørgensen (1963): 18-20 & Pol. & V-VI & Gyttja, peat \\
\hline 536 & Frihedslund & Brøndsted (1938): 362 & Arch. & NA & Imprint \\
\hline 537 & Niløse, Åmosen & Jørgensen (1963): 24-25 & Pol. & VI & Gyttja, peat \\
\hline 538 & Ruds-Vedby & Krog (1954): 130-135 & C-14, Pol. & $\begin{array}{l}\text { Zone limit II/ } \\
\text { III: } 11030 \pm 200 \\
\text { B.P. }\end{array}$ & Clay, gyttja \\
\hline 539 & Maglemose & Jessen $(1935 b): 12$ & $\begin{array}{l}\text { Arch., } \\
\text { Pol. }\end{array}$ & $\mathrm{Vu}$ & Gyttja \\
\hline 540 & Bromme & Iversen (1946): 222 & Pol & III & Sand \\
\hline 541 & Solrød & Hatt (1937): 36 & Arch. & LBA & Imprint \\
\hline 542 & Store Valby & Helbæk (1955 a): 198-200 & Arch. & ENA & Imprint \\
\hline 543 & Trelleborg & Jessen (1948): 168-171 & Pol. & VIII, VA & Gyttja, sand \\
\hline 544 & Fredsgårde & Brøndsted (1938): 358 & Arch. & MNA & Imprint \\
\hline 545 & Herfølge & Milthers (1908): $225-226$ & Mac. & I u & Clay, sand \\
\hline 546 & Testrup & Milthers (1908): 229 & Mac. & II u & Gyttja \\
\hline 547 & Troelstrup & Milthers (1908): 228 & Mac. & II $\mathbf{u}$ & Gyttja \\
\hline 548 & Lystrup, Faxe & Milthers (1908): 225 & Mac. & I u & Clay \\
\hline 549 & Holmegård & Jessen (1935 b): $15-16$ & Pol. & $\mathrm{V}-\mathrm{VI}$ & Gyttja, peat \\
\hline 550 & Holmegård & Jessen $(1924 b): 18-19$ & Arch. & V-VI & Gyttja, peat \\
\hline 551 & Kongsted Lyng & Milthers (1908): 234 & Mac. & VII u-VIII u & Gyttja \\
\hline 552 & Herlufsholm & $\emptyset$ dum (1965): 53 & Arch. & Abt. 1400 A.D. & Germination \\
\hline 553 & Åderup Eng & Jessen (1920): 169-170 & Arch., Pol. & VII u, GIA & Gyttja \\
\hline
\end{tabular}




\begin{tabular}{|c|c|c|c|c|c|}
\hline $\begin{array}{l}\text { Site } \\
\text { number }\end{array}$ & Site & $\begin{array}{l}\text { Author, year of publication, } \\
\text { page(s) }\end{array}$ & Dating & Period & Medium \\
\hline 554 & Brandelev & Milthers (1908): 230 & Mac. & II u & Sand \\
\hline 555 & Sværdborg & Jessen $(1935 b): 22$ & Pol. & V-VI & Gyttja, peat \\
\hline 556 & Even & Mikkelsen (1949): 52-69 & Pol. & V-IX & Gyttja, peat \\
\hline 557 & $\begin{array}{l}\text { Ræveholms } \\
\text { Mose }\end{array}$ & Mikkelsen (1949): 77 & Pol. & VIII-IX & Gyttja, peat \\
\hline 558 & Orup Mose & Mikkelsen (1949): 89-90 & Pol. & VII-IX & Gyttja, peat \\
\hline 559 & Vandmose & Jessen (1920): 127-128 & Pol. & VIII-IX & Gyttja, peat \\
\hline 560 & Tårbæk & Jessen (1920): 238 & Mac. & VII u & - \\
\hline 561 & Holmegård & Nilsson (1948): 205 & Pol. & IV-IX & Gyttja, peat \\
\hline 562 & Lundby & Jessen $(1935, b): 24-28$ & Pol. & II-III, V-VI & Clay, gyttja, peat \\
\hline 563 & Nebbele, Stege & Brøndsted (1938): 358 & Arch. & MNA & Imprint \\
\hline 564 & $\begin{array}{l}\text { Tyste Mose, } \\
\text { Rejnstrup }\end{array}$ & Mikkelsen (1984): 11 & Pol. & PRIA-LMA & Peat \\
\hline 601 & Găbense & Brøndsted (1938): 359 & Arch. & NA & Imprint \\
\hline 602 & Juellinge & Gram (1911): $40-46$ & Arch. & RIA & Grave \\
\hline 603 & Tåderup & $\emptyset$ dum (1920): 11 & Pol. & $\mathrm{V}$ u-VIII u & Gyttja, peat \\
\hline 604 & Grænge Mose & Andersen \& Møller (1946): 4-9 & Pol. & IV-V & Gyttja, peat \\
\hline 605 & $\begin{array}{l}\text { Flintinge } \\
\text { Byskov }\end{array}$ & Brøndsted (1938): 358 & Arch. & NA & Imprint \\
\hline 606 & Nagelsti & Rostrup (1877): 79-80 & Arch. & BA & Vessel \\
\hline 607 & Nagelsti & Sarauw (1900): 98 & Arch. & $\mathrm{BA}$ & Vessel \\
\hline 608 & Udstolpe & Brøndsted (1938): 358 & Arch. & NA & Imprint \\
\hline 609 & Lidsø, Rødby & $\begin{array}{l}\text { Jørgensen \& Fredskild (1978): 189- } \\
192\end{array}$ & Arch. & MNA & Pit \\
\hline 701 & $\begin{array}{l}\text { Vestergård, } \\
\text { Rutsker }\end{array}$ & Hartz (1902): 46 & Mac. & III u & Clay \\
\hline 702 & $\begin{array}{l}\text { Nørre } \\
\text { Sandegård }\end{array}$ & Helbæk (1952 b): $108-110$ & Arch. & EBA & Pit \\
\hline 703 & $\begin{array}{l}\text { Nørre } \\
\text { Sandegård }\end{array}$ & Helbæk (1958 c): 118-127 & Arch. & EBA & Pit \\
\hline 704 & $\begin{array}{l}\text { Skinderbygård } \\
\text { Mose }\end{array}$ & Hartz (1902): 48 & Mac. & III u & Clay \\
\hline 705 & $\begin{array}{l}\text { Skinderbygård } \\
\text { Mose }\end{array}$ & $\begin{array}{l}\text { Grönwall \& Milthers (1916): 230- } \\
231\end{array}$ & Mac. & I-III & Clay, gyttja \\
\hline 706 & Kalvemose & Grönwall \& Milthers (1916): 226 & Mac. & II-III & Clay, gyttja \\
\hline 707 & Dalshøj & Helbæk $(1958$ c): $122-127$ & Arch. & 1st cent. A.D. & Storage \\
\hline 708 & Dalshøj & Helbæk (1957): 261-269 & Arch. & 1st cent. A.D. & Storage \\
\hline 709 & Lilleborg & Helbæk (1953): 10-15 & Arch. & 1259 A.D. & Storage \\
\hline 710 & Lilleborg & Helbæk (1958 c): 124-127 & Arch. & 1259 A.D. & Storage \\
\hline 711 & Græssöen & Mikkelsen (1954): 225 & Pol. & IV-IX & Gyttja, peat \\
\hline 712 & Sorte Muld & Helbæk (1957): $261-269$ & Arch. & Abt. 500 A.D. & Burnt house \\
\hline 713 & Sorte Muld & Helbæk $(1958 \mathrm{c}):$ 123-127 & Arch. & Abt. 500 A.D. & Burnt house \\
\hline 714 & $\begin{array}{l}\text { Vallensgård } \\
\text { Mose }\end{array}$ & Grönwall \& Milthers (1916): 222 & Mac. & I-III & Clay, gyttja, sand \\
\hline 715 & Vallensgård & Hatt (1937): 37 & Arch. & NA & Imprint \\
\hline 716 & Vallensgård & Brøndsted (1938): 139-141 & Arch. & NA & Imprint \\
\hline 717 & $\begin{array}{l}\text { Øster } \\
\text { Krusegård }\end{array}$ & Brøndsted (1938): 358 & Arch. & NA & Imprint \\
\hline 718 & $\begin{array}{l}\text { Dovr } \AA s, \\
\text { Rønne }\end{array}$ & Jessen \& Lind (1922-23): 18 & Arch. & $300-500$ A.D. & Imprint \\
\hline 719 & $\begin{array}{l}\text { Søhjem, } \\
\emptyset \text { stermarie }\end{array}$ & Grönwall \& Milthers (1916): 225 & Mac. & I-III & $\begin{array}{l}\text { Clay, gyttja, sandy } \\
\text { clay }\end{array}$ \\
\hline 720 & Anhøj Mose & Grönwall \& Milthers (1916): 224 & Mac. & I-III & Clay, gyttja \\
\hline 801 & Vallda & Hjelmqvist (1955 a): 94 & Arch. & PRIA & Imprint \\
\hline 802 & Onsala & Hjelmqvist (1960): 152-154 & Arch. & PRIA & Imprint \\
\hline 803 & Grimeton & Hjelmqvist (1955 a): 94 & Arch. & RIA u & Imprint \\
\hline 804 & Köinge & Hjelmqvist (1955 a): 94 & Arch. & RIA u & Imprint \\
\hline 805 & Vessige & Hjelmqvist (1955 a): 94 & Arch. & RIA u & Imprint \\
\hline 806 & Årstad & Hjelmqvist (1955 a): 94 & Arch. & RIA & Imprint \\
\hline 807 & Övraby & Hjelmqvist (1955 a): 94 & Arch. & RIA & Imprint \\
\hline 808 & Halmstad & Hjelmqvist (1971-72): 149 & Arch. & EBA & Imprint \\
\hline 809 & Halmstad & Hjelmqvist (1972): 70-75 & Arch. & LMA & Burnt layer \\
\hline
\end{tabular}




\begin{tabular}{|c|c|c|c|c|c|}
\hline $\begin{array}{l}\text { Site } \\
\text { number }\end{array}$ & Site & $\begin{array}{l}\text { Author, year of publication, } \\
\text { page(s) }\end{array}$ & Dating & Period & Medium \\
\hline 810 & Halmstad & Hjelmqvist (1979): 24 & Arch. & EBA & Imprint \\
\hline 811 & Eldsberga & Hjelmqvist (1955 a): 94 & Arch. & RIA & Imprint \\
\hline 812 & Ysby & Hjelmqvist (1979): 50 & Arch. & RIA u & Imprint \\
\hline 813 & Hasslöv & Hjelmqvist (1955 a): 94 & Arch. & IA u & Imprint \\
\hline 814 & Augerum & Hjelmqvist (1955 a): 92 & Arch. & VA & Imprint \\
\hline 815 & S. Svartasjön & Sandegren (1931-32): $236-240$ & Pol. & V-VI & Gyttja \\
\hline 816 & S. Svartasjön & Sandegren (1941): 62 & - & $\mathrm{V} \mathbf{u}$ & - \\
\hline 817 & Ronneby & Hjelmqvist (1955 a): 92 & Arch. & GIA-VA & Imprint \\
\hline \multirow[t]{2}{*}{818} & Hallarums & Berglund (1964): 16-17 & Pol., C-14 & $5370 \pm 90$ B.C. & Gyttja \\
\hline & Mosse & & & $\begin{array}{l}5210 \pm 90 \text { B.C. } \\
3240 \pm 85 \text { B.C. }\end{array}$ & \\
\hline 819 & Mörrum & Hjelmqvist (1955 a): 59 & Arch. & LBA & Imprint \\
\hline 820 & Ysane & Sandegren (1931-32): 236 & Pol. & VI-VII & Gyttja \\
\hline 821 & Ysane & Sandegren (1941): 62 & - & VII u & - \\
\hline \multirow[t]{2}{*}{822} & Inlängans & Berglund (1964): 31 & Pol., C-14 & $1700 \pm 85$ B.C. & Gyttja \\
\hline & Mosse & & & & \\
\hline 823 & Mjällby & Hjelmqvist (1955 a): 92 & Arch. & IA u & Imprint \\
\hline 824 & Mjällby & Hjelmqvist (1955 a): 9 & Arch. & ENA & Imprint \\
\hline 825 & Mjällby & Hjelmqvist (1979): 19 & Arch. & ENA & Imprint \\
\hline \multirow[t]{2}{*}{826} & Eastern & Berglund (1966 a): 185-190 & C-14, Pol. & V-IX & Clay, gyttja, peat \\
\hline & Blekinge & & & & \\
\hline \multirow[t]{2}{*}{827} & Eastern & Berglund (1966 b): 175-179 & C-14, Pol. & II-IX & Clay, gyttja, peat \\
\hline & Blekinge & & & & \\
\hline 901 & Hov & Hjelmqvist (1955 a): 57 & Arch. & LBA & Imprint \\
\hline 902 & Grevie & Hjelmqvist (1955 a): 37 & Arch. & LNA & Imprint \\
\hline 903 & $\begin{array}{l}\text { Grevie: } \\
\text { Ängelsbäck }\end{array}$ & Hjelmqvist (1955 a): 57 & Arch. & LBA & Imprint \\
\hline 904 & $\begin{array}{l}\text { Grevie: } \\
\text { Ängelsbäck }\end{array}$ & Hjelmqvist (1953): 422 & Arch. & $\mathrm{BA}$ & Imprint \\
\hline 905 & Immeln & Digerfeldt (1974): Diagram MBP & Pol., C-14 & III-IX & Clay, gyttja \\
\hline 906 & Immeln & $\begin{array}{l}\text { Digerfeldt (1966): Diagram BP1, } \\
\text { BP2 }\end{array}$ & Pol. & VII-IX & Gyttja \\
\hline 907 & Ö. Broby & Hjelmqvist (1955 a): 87 & Arch. & GIA-VA & Imprint \\
\hline 908 & Emislöv & Hjelmqvist (1955 a): 87 & Arch. & IA u & Imprint \\
\hline 909 & Jonstorp & Hjelmqvist (1955 a): 20 & Arch. & MNA & Imprint \\
\hline 910 & Jonstorp & Hjelmqvist (1964): 34 & Arch. & MNA & Imprint \\
\hline 911 & Emislöv & Hjelmqvist (1955 a): 57 & Arch. & LBA & Imprint \\
\hline 912 & Välinge & Hjelmqvist (1955 a): 58 & Arch. & LBA & Imprint \\
\hline 913 & Gryt & Hjelmqvist (1955 a): 57 & Arch. & LBA & Imprint \\
\hline 914 & Fjälkestad & Hjelmqvist (1955 a): 57 & Arch. & LBA & Imprint \\
\hline 915 & Fjälkestad & Hjelmqvist (1964): 34 & Arch. & MNA & Imprint \\
\hline 916 & Stoby & Hjelmqvist (1955 a): 58 & Arch. & LBA & Imprint \\
\hline 917 & Gråmanstorp & Hjelmqvist (1955 a): 57 & Arch. & LBA & Imprint \\
\hline 918 & Gråmanstorp & Hjelmqvist (1979): 37 & Arch. & LBA & Imprint \\
\hline 919 & Färlöv & Hjelmqvist (1955 a): 87 & Arch. & IA u & Imprint \\
\hline 920 & Ivö & Hjelmqvist (1955 a): 57 & Arch. & LBA & Imprint \\
\hline 921 & Ivetofta & Hjelmqvist (1955 a): 20 & Arch. & MNA & Imprint \\
\hline 922 & Bromölla & Hjelmqvist (1979): 29, 37 & Arch. & LBA & Imprint \\
\hline 923 & Bromölla & Hjelmqvist (1969 a): $260-262$ & Arch. & LBA & Imprint \\
\hline 924 & Kiaby & Hjelmqvist $(1955 \mathrm{a}): 87$ & Arch. & IA u & Imprint \\
\hline 925 & Nosaby & Hjelmqvist (1955 a): 20,37 & Arch. & $\begin{array}{l}\text { MNA, LNA, } \\
\text { IA u }\end{array}$ & Imprint \\
\hline 926 & Nosaby & Hjelmqvist (1979): 19 & Arch. & ENA & Imprint \\
\hline 927 & Nosaby & Hjelmqvist (1952): 334 & Arch. & MNA & Imprint \\
\hline 928 & Riseberga: & Hjelmqvist (1955 a): 58 & Arch. & LBA & Imprint \\
\hline & Bonarp & & & & \\
\hline 929 & $\begin{array}{l}\text { N. Mellby: } \\
\text { Maglö }\end{array}$ & Hjelmqvist (1955 a): 20 & Arch. & MNA & Imprint \\
\hline 930 & $\begin{array}{l}\text { N. Mellby: } \\
\text { Maglö }\end{array}$ & Hjelmqvist (1964): 34 & Arch. & MNA & Imprint \\
\hline 931 & Skepparslöv & Hjelmqvist (1955 a): 87 & Arch. & IA u & Imprint \\
\hline 932 & Fjälkinge & Hjelmqvist (1955 a): 57 & Arch. & LBA & Imprint \\
\hline
\end{tabular}


Table 1 continued

\begin{tabular}{|c|c|c|c|c|c|}
\hline $\begin{array}{l}\text { Site } \\
\text { number }\end{array}$ & Site & $\begin{array}{l}\text { Author, year of publication, } \\
\text { page(s) }\end{array}$ & Dating & Period & Medium \\
\hline 933 & N. Mellby & Hjelmqvist (1962): 911 & Arch. & MNA & Imprint \\
\hline 934 & Färingtofta & Sandegren (1941): 61 & Pol. & $\mathrm{V}$ & Gyttja, peat \\
\hline 935 & Vä & Hjelmqvist (1955 a): 58,87 & Arch. & LBA, RIA & Imprint \\
\hline 936 & Vä & Hjelmqvist (1964): 35 & Arch. & PRIA-RIA & Imprint \\
\hline 937 & $\begin{array}{l}\text { Riseberga: } \\
\text { Tostarp }\end{array}$ & Hjelmqvist (1955 a): 58 & Arch. & LBA & Imprint \\
\hline 938 & Vätteryd & Hjelmqvist (1979): 19 & Arch. & ENA & Imprint \\
\hline 939 & Vätteryd & Hjelmqvist (1964): 34 & Arch. & ENA & Imprint \\
\hline 940 & Vätteryd & Hjelmqvist (1958 a): 103-106 & Arch. & ENA & Imprint \\
\hline 941 & Nymö & Hjelmqvist (1955 a): 58 & Arch. & LBA & Imprint \\
\hline 942 & Raus & Hjelmqvist (1955 a): 58 & Arch. & LBA & Imprint \\
\hline 943 & $\begin{array}{l}\text { Trolle-Ljungby: } \\
\text { Hammaren }\end{array}$ & Hjelmqvist (1952): 334 & Arch. & MNA & Imprint \\
\hline 944 & Ageröds Mosse & Nilsson (1964): 17-19 & Pol. & IV-IX & Gyttja, peat \\
\hline 945 & Västra Vram & Hjelmqvist (1955 a): 87 & Arch. & IA u & Imprint \\
\hline 946 & $\begin{array}{l}\text { Glumslöv: } \\
\text { Viktorshög }\end{array}$ & Hjelmqvist (1955 a): 20 & Arch. & MNA & Imprint \\
\hline 947 & $\begin{array}{l}\text { Glumslöv: } \\
\text { Örenäs }\end{array}$ & Hjelmqvist (1955 a): 20 & Arch. & MNA & Imprint \\
\hline 948 & Asmundtorp & Hjelmqvist (1955 a): 57, 87 & Arch. & $\begin{array}{l}\text { LBA, PRIA- } \\
\text { RIA }\end{array}$ & Imprint \\
\hline 949 & Asmundtorp & Hjelmqvist (1979): 19 & Arch. & ENA & Imprint \\
\hline 950 & Asmundtorp & Hjelmqvist (1964): 34 & Arch. & ENA & Imprint \\
\hline 951 & Råga Hörstad & Hjelmqvist (1979): 52 & Arch. & PRIA & Imprint \\
\hline 952 & Lyby Mosse & Magnusson (1962): 9-22 & Pol. & IV-VII & Gyttja, peat \\
\hline 953 & Landskrona & Hjelmqvist (1955 a): 57 & Arch. & EBA & Imprint \\
\hline 954 & Landskrona & Hjelmqvist (1979): 24 & Arch. & EBA & Imprint \\
\hline 955 & Landskrona & Hjelmqvist (1953): 423 & Arch. & EBA & Imprint \\
\hline 956 & Landskrona & Hjelmqvist (1971-72): 149 & Arch. & EBA & Imprint \\
\hline 957 & Landskrona & $\begin{array}{l}\text { Hjelmqvist (1968 a): } \\
\text { 182-188 }\end{array}$ & Arch. & LMA & Imprint, storage \\
\hline 958 & Norrvidinge & Hjelmqvist (1979): 24 & Arch. & EBA & Imprint \\
\hline 959 & Norrvidinge & Hjelmqvist (1971-72): 149 & Arch. & EBA & Imprint \\
\hline 960 & Saxtorp & Hjelmquist (1955 a): 87 & Arch. & IA u & Imprint \\
\hline 961 & Annelöv & Hjelmqvist (1955 a): 57 & Arch. & LBA & Imprint \\
\hline 962 & Södervidinge & Hjelmquist (1955 a): 20 & Arch. & MNA & Imprint \\
\hline 963 & Södervidinge & Hjelmqvist (1952): 331 & Arch. & MNA & Imprint \\
\hline 964 & Dösjöbro & Hjelmqvist (1979): 19 & Arch. & ENA & Imprint \\
\hline 965 & Västra Hoby & Hjelmqvist (1955 a): 20 & Arch. & MNA & Imprint \\
\hline 966 & Västra Hoby & Hjelmqvist (1964): 34 & Arch. & MNA & Imprint \\
\hline 967 & Västra Hoby & Hjelmqvist (1955 b): 289 & Arch. & NA & Imprint \\
\hline 968 & Barsebäck & Hjelmqvist (1955 a): 57 & Arch. & LBA & Imprint \\
\hline 969 & Barsebäck & Hjelmqvist (1953): $422-425$ & Arch. & BA, LBA & Imprint \\
\hline 970 & Barsebäck & Hjelmqvist (1964): 28 & Arch. & LBA & Imprint \\
\hline 971 & Lackalänga & Hjelmqvist (1955 a): 20 & Arch. & MNA & Imprint \\
\hline 972 & Lackalänga & Hjelmqvist (1952): 335 & Arch. & MNA & Imprint \\
\hline 973 & $\begin{array}{l}\text { Ö. Vem- } \\
\text { menhög }\end{array}$ & Hjelmqvist (1955 a): 87 & Arch. & PRIA & Imprint \\
\hline 974 & $\begin{array}{l}\text { Barsebäck: } \\
\text { Storegården }\end{array}$ & Hjelmqvist (1955 a): 20 & Arch. & MNA & Imprint \\
\hline 975 & Barsebäck & Hjelmqvist (1979): 37 & Arch. & LBA & Imprint \\
\hline 976 & $\begin{array}{l}\text { Barsebäck: } \\
\text { Gillhög }\end{array}$ & Hjelmqvist (1955 a): 20 & Arch. & MNA & Imprint \\
\hline 977 & $\begin{array}{l}\text { Barsebäck: } \\
\text { Gillhög }\end{array}$ & Hjelmqvist (1953): 421 & Arch. & MNA & Imprint \\
\hline 978 & $\begin{array}{l}\text { Barsebäck: } \\
\text { Gillhög }\end{array}$ & Hjelmqvist (1964): 34 & Arch. & MNA & Imprint \\
\hline 979 & $\begin{array}{l}\text { Barsebäck: } \\
\text { Gillhög }\end{array}$ & Hjelmqvist (1952): 333 & Arch. & MNA & Imprint \\
\hline 980 & Hardeberga & Hjelmqvist (1955 a): 87 & Arch. & RIA & Imprint \\
\hline 981 & Löddeköpinge & Hjelmqvist (1955 a): 58 & Arch. & LBA & Imprint \\
\hline 982 & Löddesborg & Hjelmqvist (1979): 19-24 & Arch. & ENA, EBA & Imprint \\
\hline
\end{tabular}


Table 1 continued

\begin{tabular}{|c|c|c|c|c|c|}
\hline $\begin{array}{l}\text { Site } \\
\text { number }\end{array}$ & Site & $\begin{array}{l}\text { Author, year of publication, } \\
\text { page(s) }\end{array}$ & Dating & Period & Medium \\
\hline 983 & Löddesborg & Hjelmqvist (1971-72): 149 & Arch. & EBA & Imprint \\
\hline 984 & Lund & Hjelmqvist (1964): 35 & Arch. & GIA-VA & Imprint \\
\hline 985 & Lund & Hjelmqvist (1963): 233-267 & Arch. & $\begin{array}{l}\text { VA, } 13 \text { th-14th } \\
\text { cent. A.D. }\end{array}$ & Storage \\
\hline 986 & Ö. Kärrstorp & Hjelmqvist (1979): 19 & Arch. & ENA & Imprint \\
\hline 987 & St. Råby & Hjelmqvist (1979): 4-19 & Arch. & ENA & Imprint \\
\hline 988 & Bjellerup & Hjelmqvist (1955 a): 57 & Arch. & LBA & Imprint \\
\hline 989 & Bjellerup & Hjelmqvist (1953): 427 & Arch. & LBA & Imprint \\
\hline 990 & $\begin{array}{l}\text { S. Mellby: } \\
\text { Kivik }\end{array}$ & Hjelmqvist (1955 a): 58-59 & Arch. & BA, LBA & Imprint \\
\hline 991 & Bjellerup & Hjelmqvist (1955 a): 20 & Arch. & MNA & Imprint \\
\hline 992 & Dalby & Hjelmqvist (1968 a): 179-182 & Arch. & $1100-1300$ A.D. & Storage \\
\hline 993 & Uppåkra & Hjelmqvist (1955 a): 87 & Arch. & RIA & Imprint \\
\hline 994 & Uppåkra & Hjelmqvist (1964): 35 & Arch. & $\begin{array}{l}\text { PRIA-RIA, } \\
\text { GIA-VA }\end{array}$ & Imprint \\
\hline 995 & Torreberga & Berglund \& Digerfeldt (1970): 106 & Pol. & III-IV & Gyttja, peat \\
\hline 996 & $\begin{array}{l}\text { S. Mellby: } \\
\text { Äsperöd }\end{array}$ & Hjelmqvist (1955 a): 58 & Arch. & LBA & Imprint \\
\hline 997 & $\begin{array}{l}\text { L. Lund, } \\
\text { Grevie }\end{array}$ & Hjelmqvist (1964): 26 & Arch. & LNA & Imprint \\
\hline 998 & Lyngby & Hjelmqvist (1955 a): 58 & Arch. & LBA & Imprint \\
\hline 999 & S. Sallerup & Hjelmqvist (1955 a): 58 & Arch. & LBA & Imprint \\
\hline 1000 & Värby & Hjelmqvist (1979): 19 & Arch. & ENA & Imprint \\
\hline 1001 & Värby & Hjelmqvist (1970): 96-98 & Arch. & ENA & Imprint \\
\hline 1002 & Malmö & Hjelmqvist (1979): 52 & Arch. & PRIA & Imprint \\
\hline 1003 & Oxie & Hjelmqvist (1955 a): 9 & Arch. & ENA & Imprint \\
\hline 1004 & Oxie & Hjelmqvist (1979): 19 & Arch. & ENA & Imprint \\
\hline 1005 & Oxie & Hjelmqvist (1964): 34 & Arch. & ENA & Imprint \\
\hline 1006 & Oxie & Hjelmqvist (1952): 330-331 & Arch. & ENA & Imprint \\
\hline 1007 & Oxie & Hjelmqvist (1958 b): 399 & Arch. & ENA & Imprint \\
\hline 1008 & Gladsax & Hjelmqvist (1979): 18 & Arch. & MNA & Imprint \\
\hline 1009 & Sturup & Hjelmqvist (1979): $6-19$ & Arch. & ENA & Imprint \\
\hline 1010 & Sturup & Hjelmqvist (1974): 215-219 & Arch. & ENA & Imprint \\
\hline 1011 & Simrishamn & Hjelmqvist (1979): 18 & Arch. & MNA & Imprint \\
\hline 1012 & Limhamn & Hjelmqvist (1955 a): 9 & Arch. & ENA & Imprint \\
\hline 1013 & Limhamn & Hjelmqvist (1979): 19 & Arch. & ENA & Imprint \\
\hline 1014 & Limhamn & Hjelmqvist (1964): 34 & Arch. & ENA & Imprint \\
\hline 1015 & Simris & Hjelmqvist (1955 a): 58,87 & Arch. & LBA, RIA & Imprint \\
\hline 1016 & Simris & Hjelmqvist (1979): 19 & Arch. & ENA & Imprint \\
\hline 1017 & Simris & Hjelmqvist (1964): 34 & Arch. & ENA & Imprint \\
\hline 1018 & Simris & Hjelmqvist (1958 b): 399 & Arch. & ENA & Imprint \\
\hline 1019 & Järrestad & Hjelmqvist (1955 a): 57 & Arch. & LBA & Imprint \\
\hline 1020 & Skabersjö & Hjelmqvist (1979): 19 & Arch. & ENA & Imprint \\
\hline 1021 & Skabersjö & Hjelmqvist (1964): 34 & Arch. & ENA & Imprint \\
\hline 1022 & $\begin{array}{l}\text { Svedala: } \\
\text { Hyltarp }\end{array}$ & Hjelmqvist (1955 a): 20 & Arch. & MNA & Imprint \\
\hline 1023 & $\begin{array}{l}\text { Svedala: } \\
\text { Hyltarp }\end{array}$ & Hjelmqvist (1964): 34 & Arch. & MNA & Imprint \\
\hline 1024 & $\begin{array}{l}\text { Svedala: } \\
\text { Hyltarp }\end{array}$ & Hjelmqvist (1952): 334 & Arch. & MNA & Imprint \\
\hline 1025 & Tommarp & $\emptyset$ dum (1965): 52-53 & Arch. & Abt. 1300 A.D. & $\begin{array}{l}\text { Germina- } \\
\text { tion }\end{array}$ \\
\hline 1026 & $\begin{array}{l}\text { Simris - } \\
\text { Brantevik }\end{array}$ & Hjelmqvist (1955 a): 59 & Arch. & $\mathrm{BA}$ & Imprint \\
\hline 1027 & Svedala & Hjelmqvist (1962): 911 & Arch. & MNA & Imprint \\
\hline 1028 & $\begin{array}{l}\text { Svedala: S. } \\
\text { Lindved }\end{array}$ & Hjelmqvist (1955 a): 9 & Arch. & ENA & Imprint \\
\hline 1029 & $\begin{array}{l}\text { Svedala: S. } \\
\text { Lindved }\end{array}$ & Hjelmqvist (1979): 19 & Arch. & ENA & Imprint \\
\hline 1030 & $\begin{array}{l}\text { Svedala: S. } \\
\text { Lindved }\end{array}$ & Hjelmqvist (1964): 34 & Arch. & ENA & Imprint \\
\hline 1031 & Hötofta & Hjelmqvist (1979): 37 & Arch. & LBA & Imprint \\
\hline
\end{tabular}


Table 1 continued

\begin{tabular}{|c|c|c|c|c|c|}
\hline $\begin{array}{l}\text { Site } \\
\text { number }\end{array}$ & Site & $\begin{array}{l}\text { Author, year of publication, } \\
\text { page(s) }\end{array}$ & Dating & Period & Medium \\
\hline 1032 & Hötofta & Hjelmqvist (1969 b): 214 & Arch. & LBA & Imprint \\
\hline 1033 & Tosterup & Hjelmqvist (1955 a): 58 & Arch. & LBA & Imprint \\
\hline 1034 & $\begin{array}{l}\text { Dolmen } \\
\text { Trollasten }\end{array}$ & Hjelmqvist (1968 b): 248 & Arch. & MNA & Imprint \\
\hline 1035 & St. Köpinge & Hjelmqvist (1979): 31 & Arch. & LBA & Imprint \\
\hline 1036 & $\begin{array}{l}\text { Bjärsjö- } \\
\text { holmssjön }\end{array}$ & Nilsson (1961): $29-30$ & Pol. & IV-IX & Gyttja, peat \\
\hline 1037 & Kvarnby & Hjelmqvist (1955 a): 87 & Arch. & GIA-VA & Imprint \\
\hline 1038 & Köpingebro & Hjelmqvist (1979): 18 & Arch. & MNA & Imprint \\
\hline 1039 & St. Herrestad & Hjelmqvist (1955 a): 9 & Arch. & ENA & Imprint \\
\hline 1040 & St. Herrestad & Hjelmqvist (1979): 19 & Arch. & ENA & Imprint \\
\hline 1041 & St. Herrestad & Hjelmqvist (1964): 34 & Arch. & ENA & Imprint \\
\hline 1042 & St. Herrestad & Hjelmqvist (1952): 331 & Arch. & ENA & Imprint \\
\hline 1043 & Ö. Hoby & Hjelmqvist $(1955 \mathrm{a}): 58$ & Arch. & LBA & Imprint \\
\hline 1044 & Ö. Hoby & Hjelmqvist (1953): 422 & Arch. & BA & Imprint \\
\hline 1045 & Bjäresjö & Hjelmqvist (1955 a): $57,59,87$ & Arch. & BA, LBA, RIA & Imprint \\
\hline 1046 & Ruuthsbo & Hjelmqvist (1953): 427 & Arch. & BA & Imprint \\
\hline 1047 & Ruuthsbo & Hjelmqvist (1964): 28 & Arch. & LBA & Imprint \\
\hline 1048 & Västra Alstad & Hjelmqvist (1955 a): 87 & Arch. & RIA & Imprint \\
\hline 1049 & Hörup & Hjelmqvist (1955 a): 20 & Arch. & MNA & Imprint \\
\hline 1050 & Skivarp & Hjelmqvist (1955 a): 58 & Arch. & LBA & Imprint \\
\hline 1051 & Skivarp & Hjelmqvist (1979): 37 & Arch. & LBA & Imprint \\
\hline 1052 & Skivarp & Hjelmqvist (1953): 423-424 & Arch. & LBA & Imprint \\
\hline 1053 & Balkåkra & Hjelmqvist (1955 a): 57 & Arch. & LBA & Imprint \\
\hline 1054 & Balkåkra & Hjelmqvist (1953): $423-425$ & Arch. & BA, LBA & Imprint \\
\hline 1055 & Svarte & Hjelmqvist (1964): 28 & Arch. & LBA & Imprint \\
\hline 1056 & Ingelstorp & Hjelmqvist (1979): 18 & Arch. & MNA & Imprint \\
\hline 1057 & Löderup & Hjelmqvist (1955 a): 20 & Arch. & MNA & Imprint \\
\hline 1058 & Löderup & Hjelmqvist (1979): 31 & Arch. & LBA & Imprint \\
\hline 1059 & Reng & Hjelmqvist (1955 a): 57 & Arch. & EBA & Imprint \\
\hline 1060 & Reng & Hjelmqvist (1979): 24 & Arch. & EBA & Imprint \\
\hline 1061 & Reng & Hjelmqvist (1971-72): 149 & Arch. & EBA & Imprint \\
\hline 1062 & $\begin{array}{l}\text { Bösarp: V. } \\
\text { Virestad }\end{array}$ & Hjelmqvist (1955 a): 57 & Arch. & LBA & Imprint \\
\hline 1063 & $\begin{array}{l}\text { Bösarp: V. } \\
\text { Virestad }\end{array}$ & Hjelmqvist (1953): 422 & Arch. & BA & Imprint \\
\hline 1064 & Valleberga & Hjelmqvist (1979): 18-47 & Arch. & $\begin{array}{l}\text { ENA, MNA, } \\
\text { RIA, GIA-VA }\end{array}$ & Imprint \\
\hline 1065 & Hagestad & Hjelmqvist (1979): 15-47 & Arch. & $\begin{array}{l}\text { MNA, LBA, } \\
\text { PRIA-RIA, } \\
\text { GIA, VA }\end{array}$ & Imprint \\
\hline 1066 & Ö. Vemmenhög & Hjelmqvist (1955 a): 58 & Arch. & LBA & Imprint \\
\hline 1067 & $\begin{array}{l}\text { Skivarp: } \\
\text { Abbekås }\end{array}$ & Hjelmqvist (1955 a): 58 & Arch. & LBA & Imprint \\
\hline 1068 & $\begin{array}{l}\text { Skivarp: } \\
\text { Abbekås }\end{array}$ & Hjelmqvist (1953): 423 & Arch. & LBA & Imprint \\
\hline 1069 & Ramshög & Hjelmqvist (1979): 18 & Arch. & MNA & Imprint \\
\hline 1070 & Reng & Hjelmqvist (1955 a): 58 & Arch. & LBA & Imprint \\
\hline 1071 & Reng & Hjelmqvist (1953): 423 & Arch. & LBA & Imprint \\
\hline 1072 & Gislöv: Åkarp & Hjelmqvist (1955 a): 20,57 & Arch. & MNA, LBA & Imprint \\
\hline 1073 & Gislöv: Åkarp & Hjelmqvist (1953): 420 & Arch. & MNA & Imprint \\
\hline 1074 & Hög & Hjelmqvist (1955 a): 20 & Arch. & MNA & Imprint \\
\hline 1075 & Maglarp & Hjelmqvist (1955 a): 9 & Arch. & ENA & Imprint \\
\hline 1076 & Maglarp & Hjelmqvist (1979): 19 & Arch. & ENA & Imprint \\
\hline 1077 & Maglarp & Hjelmqvist (1953): 421 & Arch. & NA & Imprint \\
\hline 1078 & Maglarp & Hjelmqvist (1964): 34 & Arch. & NA & Imprint \\
\hline 1079 & Maglarp & Hjelmqvist (1952): 331 & Arch. & NA & Imprint \\
\hline 1080 & Valleberga & Hjelmqvist (1955 a): 87 & Arch. & VA & Imprint \\
\hline 1081 & Valleberga & Hjelmqvist (1964): 35 & Arch. & GIA-VA & Imprint \\
\hline 1082 & Skateholm & Sandegren (1941): 61 & - & $\mathrm{V} \mathbf{u}$ & - \\
\hline 1083 & L. Bedinge & Hjelmqvist (1955 a): 57 & Arch. & LBA & Imprint \\
\hline 1084 & L. Isie & Hjelmqvist (1955 a): 20,57 & Arch. & MNA, LBA & Imprint \\
\hline
\end{tabular}

(continued) 
Table 1 continued

\begin{tabular}{|c|c|c|c|c|c|}
\hline $\begin{array}{l}\text { Site } \\
\text { number }\end{array}$ & Site & $\begin{array}{l}\text { Author, year of publication, } \\
\text { page(s) }\end{array}$ & Dating & Period & Medium \\
\hline 1085 & L. Isie & Hjelmqvist (1953): 422 & Arch. & BA & Imprint \\
\hline 1086 & Östra Torp & Hjelmqvist (1955 a): 20 & Arch. & MNA & Imprint \\
\hline 1087 & Gårdlösa & Hjelmqvist (1981): 57 & $\begin{array}{l}\text { Arch., } \\
\text { C-14 }\end{array}$ & RIA-VA & Imprint \\
\hline 1088 & Gårdlösa & Hjelmqvist (1979): 52-53 & Arch. & RIA, GIA, VA & Imprint \\
\hline 1089 & Stävie & Hjelmqvist (1981-82): 108-113 & Arch. & MNA & Imprint \\
\hline 1090 & $\begin{array}{l}\text { Lilla Loshults } \\
\text { Mosse }\end{array}$ & Nilsson (1968): 538 & Pol. & $\mathrm{V}$ & Gyttja, peat \\
\hline 1091 & Färingtofta & Nilsson (1958): 73-74 & Pol. & IX & Gyttja, peat \\
\hline 1092 & Gryts Socken & Hjelmqvist (1964): 28 & Arch. & LBA & Imprint \\
\hline 1093 & Nosaby & Hjelmqvist (1964): 26 & Arch. & LNA & Imprint \\
\hline 1094 & Raus & Hjelmqvist (1953): 422 & Arch. & $\mathrm{BA}$ & Imprint \\
\hline 1095 & $\begin{array}{l}\text { Barsebäck } \\
\text { Socken }\end{array}$ & Hjelmqvist (1953): 424 & Arch. & $\mathrm{BA}$ & Imprint \\
\hline 1096 & Löderup & Hjelmqvist (1953): 421-422 & Arch. & NA & Imprint \\
\hline 1097 & Valleberga & Hjelmqvist (1979): 49 & Arch. & VA & Imprint \\
\hline
\end{tabular}




\section{Table 2.}

Records of macrofossils in the reviewed literature, arranged according to the species and according to age of materials examined. Information on finds from neighbouring countries is included.

I-IX: Pollen assemblage zones, PRIA: Pre-Roman Iron Age, RIA: Roman Iron Age, GIA: Germanic Iron Age, VA: Viking Age, EMA: Early Middle Ages, LMA: Late Middle Ages (see Fig. 1).

r: 1-2 macrofossils, +: 3-20 macrofossils, c: more than 20 macrofossils reported, u: dating uncertain, cf: species uncertainly identified. BI: British Isles, NL: The Netherlands, BRD: German Federal Republic, DDR: German Democratic Republic, P: Poland, S: Sweden, N: Norway.

\begin{tabular}{|c|c|c|}
\hline Species & $\begin{array}{l}\text { Period, site number (cf. Table } 1 \text { ) and number } \\
\text { of macrofossils reported }\end{array}$ & $\begin{array}{l}\text { Reports on finds from neighbour countries. } \\
\text { Pollen zones and other periods are presented } \\
\text { as published by the authors cited }\end{array}$ \\
\hline Acer platanoides $\mathbf{L}$. & $\begin{array}{l}\text { VI, VII: } 422 \text { ru } 501+\text { VIII: } 501+508 \text { ru IX: } \\
501 \mathrm{r} 502+513 \mathrm{c} 559 \mathrm{r}\end{array}$ & \\
\hline Achillea millefolium L. & $\begin{array}{l}\text { VIII: 220r PRIA: 115r EMA: 172r LMA: } \\
218+809 r\end{array}$ & BI: II-III (Godwin 1975) \\
\hline Achillea ptarmica L. & VA: $304 r$ 334r & \\
\hline Actaea spicata L. & VI, VII: 549r & \\
\hline Aegopodium podagraria $\mathrm{L}$. & $\begin{array}{l}\text { PRIA: 301r RIA: 301c 311r VA: } 110+307+ \\
\text { 334r }\end{array}$ & \\
\hline Aethusa cynapium L. & $\begin{array}{l}\text { GIA: } 244+\text { VA: } 110+427+985 \text { r EMA: } 428 \text { r } \\
431+\text { LMA: } 430+521 \text { ru } 1025 \text { r }\end{array}$ & $\begin{array}{l}\text { BI: VI-VIII (Godwin 1975), Late 2nd } \\
\text { century A.D. (Wilson 1979) NL: } 500 \text { B.C.- } \\
\text { 200 A.D. (Zeist 1974) BRD: 12-8 B.C. } \\
\text { (Kučan 1981), 1st century A.D. (Knörzer } \\
\text { 1970), 3rd century A.D. (Lange 1973) }\end{array}$ \\
\hline Agrimonia eupatoria $\mathbf{L}$. & VIII: $965 \mathrm{r}$ & P: Neolithic (Gluza \& Wasylikowa 1977) \\
\hline Agrostemma githago $\mathrm{L}$. & $\begin{array}{l}\text { RIA: } 216+\text { GIA: } 301 \text { r } 712+\text { VA: } 103+110+ \\
\text { 172r 202c 301+ 304c 307+ 312r 334c } 985 \mathrm{c} \\
\text { 1037ru 1097r EMA: } 172 \text { r } 245 \text { c } 428+429+ \\
521+\text { u } 709+992 \text { c LMA: } 218 \text { c } 241+406 \text { c } 427+ \\
\text { 521ru } 522+809 \text { r }\end{array}$ & $\begin{array}{l}\text { BI: Roman Period and later (Godwin 1975, } \\
\text { Wilson 1978, 1979) NL: 1st-3rd century A. } \\
\text { D. (Zeist 1974) BRD: Bronze Age (Behre } \\
\text { 1982), 12-8 B.C. (Kučan 1981), 3rd century } \\
\text { A.D. (Lange 1973) P: Neolithic, Hallstatt } \\
\text { and later (Gluza \& Wasylikowa 1977) }\end{array}$ \\
\hline Agrostis sp. & $\begin{array}{l}\text { VIII: 609r RIA: 311c GIA: } 242 \text { r 244+ VA: } \\
\text { 304c 307c 334c }\end{array}$ & $\begin{array}{l}\text { NL: } 3400-3300 \text { B.C. (Zeist \& Palfenier- } \\
\text { Vegter 1981) }\end{array}$ \\
\hline Ajuga reptans $\mathrm{L}$. & VA: $304 \mathrm{r}$ 334r $564 \mathrm{r}$ EMA: $564 \mathrm{r}$ & $\begin{array}{l}\text { BI: VIIa-VIII (Godwin 1975) BRD: } \\
\text { Neolithic (Averdieck 1980), 3000-900 B.C. } \\
\text { (Stalling 1983), 12-8 B.C. (Kučan 1981), 2nd } \\
\text { century A.D. (Knörzer 1979b), c. 10th } \\
\text { century A.D. (Willerding 1973) }\end{array}$ \\
\hline Alchemilla sp. & RIA: $311 \mathrm{r}$ & BI: I-II, Roman Period (Godwin 1975) \\
\hline Alisma plantago-aquatica L. & $\begin{array}{l}\text { IV: 104r V: 104+ VIII: 531r RIA: 301c GIA: } \\
\text { 243+ 301r VA: 202+ 334+ EMA: 521ru } \\
\text { LMA: 430c 521+ u }\end{array}$ & \\
\hline $\begin{array}{l}\text { Alnus glutinosa (L.) } \\
\text { Gaertner }\end{array}$ & $\begin{array}{l}\text { IV: } 104+502+\text { V: } 104 \mathrm{c} 502+562+\text { VI, VII: } \\
410 \mathrm{c} 502+\mathrm{u} 549+562+159+164 \mathrm{r} 410 \mathrm{c} 422 \mathrm{cu} \\
502+508 \mathrm{ru} 513+\mathrm{u} 520 \mathrm{ru} 551 \mathrm{cu} 553 \mathrm{ru} 944 \mathrm{r} \\
1036 \mathrm{r} \text { VIII: } 207 \mathrm{r} 213 \mathrm{ru} 219 \mathrm{r} 322+323 \mathrm{r} 328 \mathrm{r} \\
410 \mathrm{c} 502+508 \mathrm{ru} 513+\mathrm{u} 551 \mathrm{cu} 603 \mathrm{ru} \text { IX: } 329 \mathrm{r} \\
501+502+513 \mathrm{c} \text { RIA: } 101+\text { GIA: } 553 \mathrm{c} 564+\mathrm{u} \\
\text { VA: } 307+334+\text { EMA: } 564 \mathrm{c} \text { LMA: } 217 \mathrm{cu} 522 \mathrm{r}\end{array}$ & $\begin{array}{l}\text { P: Mesolithic, Iron Age (Gluza \& } \\
\text { Wasylikowa 1977) }\end{array}$ \\
\hline $\begin{array}{l}\text { Alnus cf. glutinosa (L.) } \\
\text { Gaertner }\end{array}$ & VI, VII: 944r VIII: 944r & \\
\hline Alnus sp. & $\begin{array}{l}\text { V: } 421+u \text { VI, VII: } 421+u \text { 537r } 180 \text { r VIII: } \\
421+u\end{array}$ & \\
\hline
\end{tabular}




\begin{tabular}{|c|c|c|}
\hline Species & $\begin{array}{l}\text { Period, site number (cf. Table 1) and number } \\
\text { of macrofossils reported }\end{array}$ & $\begin{array}{l}\text { Reports on finds from neighbour countries. } \\
\text { Pollen zones and other periods are presented } \\
\text { as published by the authors cited }\end{array}$ \\
\hline Alopecurus geniculatus L. & RIA: $311+$ GIA: 242 r VA: 304 r $307+334$ r & $\begin{array}{l}\text { BI: Late 2nd century A.D. (Wilson 1979) } \\
\text { NL: 600-400 B.C. (Zeist 1974) BRD: 1st- } \\
\text { 2nd century A.D. (Körber-Grohne 1967, } \\
1979 \text { b) }\end{array}$ \\
\hline Althaea officinalis $\mathrm{L}$. & VA: $307 \mathrm{c}$ & $\begin{array}{l}\text { BI: VIIa-VIIb (Godwin 1975) NL: } 600-400 \\
\text { B.C. (Zeist 1974) }\end{array}$ \\
\hline Ammophila arenaria (L.) Link & RIA: $139 \mathrm{c}$ & \\
\hline Anagallis arvensis $\mathrm{L}$. & $\begin{array}{l}\text { RIA: 306r 311r VA: } 334 \text { r EMA: } 428+429+ \\
\text { 431c LMA: } 427+429+\end{array}$ & $\begin{array}{l}\text { BI: II, VIIb-VIII (Godwin 1975), c. } 300 \\
\text { A.D. (Wilson 1978) NL: } 500 \text { B.C.-200 A.D. } \\
\text { (Zeist 1974) BRD: Hallstatt and c. } 200 \text { B.C. } \\
\text { (Knörzer 1971c, 1979a), 12-8 B.C. (Kǔcan } \\
\text { 1981), 1st-2nd century (Körber-Grohne } \\
\text { 1967) P: Hallstatt and later (Gluza \& } \\
\text { Wasylikowa 1977) }\end{array}$ \\
\hline $\begin{array}{l}\text { Anchusa arvensis (L.) Bieb. } \\
\text { (Lycopsis arvensis L.) }\end{array}$ & VA: $304+312$ r 334+ LMA: 406r & \\
\hline Anchusa officinalis $\mathbf{L}$. & EMA: $429+431+$ & BRD: 1st century A.D. (Knörzer 1970) \\
\hline Andromeda polifolia L. & VIII: $331 \mathrm{r}$ IX: $176 \mathrm{c} 331+332+$ GIA: $242 \mathrm{r}$ & $\begin{array}{l}\text { BI: Late Weichselian, VIIb-VIII (Godwin } \\
\text { 1975) }\end{array}$ \\
\hline cf. Andromeda polifolia L. & RIA: $301 \mathrm{r}$ & \\
\hline Anethum graveolens $\mathrm{L}$. & EMA: $428+$ LMA: $241+406 c 427+$ & $\begin{array}{l}\text { BI: 1st-2nd century A.D. (Willcox 1977) } \\
\text { NL: 9th-12th century (Zeist \& Palfenier- } \\
\text { Vegter 1979) BRD: 12-8 B.C. (Kŭcan 1981), } \\
\text { 1st century A.D. (Knörzer 1967c, 1970), c. } \\
\text { 200 A.D. (Körber-Grohne 1979b) }\end{array}$ \\
\hline Angelica archangelica $L$. & VA: $334 \mathrm{r}$ & \\
\hline Angelica sylvestris $\mathrm{L}$. & VA: $334 r$ & $\begin{array}{l}\text { BI: II-III, VIII (Godwin 1975) BRD: 1st- } \\
\text { 2nd century A.D. (Körber-Grohne 1967) }\end{array}$ \\
\hline Anthemis arvensis $L$. & $\begin{array}{l}\text { GIA: } 242 \text { r } 244+\text { VA: } 172 \text { r } 334 \text { c } 985 \text { r EMA: } \\
\text { 172+ } 245+429+521+\text { u LMA: } 245+427+ \\
\text { 521ru } 522 \text { r }\end{array}$ & $\begin{array}{l}\text { NL: } 1000 \text { B.C. (Pals 1977) BRD: 1st century } \\
\text { A. D. (Knörzer 1967a, 1970) }\end{array}$ \\
\hline Anthemis cotula L. & EMA: $245+429+521+$ u LMA: $430+809+$ & $\begin{array}{l}\text { BI: Roman period (Godwin 1975), 100-300 } \\
\text { A.D. (Wilson 1968), 4th-5th century A.D. } \\
\text { (Greig 1976), 10th century A.D. (Wilson } \\
\text { 1975, Green 1978) P: 9th-12th century A.D. } \\
\text { (Wasylikowa 1978) N: Late Viking } \\
\text { (Tallantire 1979) }\end{array}$ \\
\hline $\begin{array}{l}\text { Anthriscus caucalis Bieb. (A. } \\
\text { vulgaris Pers., non Bernh.) }\end{array}$ & VA: $202+$ LMA: $521 \mathrm{ru}$ & N: 1000-1100 A.D. (Griffin 1975) \\
\hline $\begin{array}{l}\text { Anthriscus sylvestris (L.) } \\
\text { Hoff. }\end{array}$ & $\begin{array}{l}\text { VIII: } 421 \text { ru VA: } 304+334+\text { EMA: } 430 c \\
\text { LMA: } 218 \text { r } 427+\end{array}$ & $\begin{array}{l}\text { NL: } 3400-3300 \text { B.C. (Zeist \& Palfenier- } \\
\text { Vegter 1981) }\end{array}$ \\
\hline $\begin{array}{l}\text { Aphanes arvensis L. (Alche- } \\
\text { milla arvensis (L.) Scop.) }\end{array}$ & $\begin{array}{l}\text { PRIA: } 115 \text { r GIA: } 306 \text { r VA: } 304 \text { r } 334+\text { EMA: } \\
\text { 245+ 521+u LMA: } 521 \text { ru }\end{array}$ & BI: PRIA (Godwin 1975) \\
\hline Aphanes sp. & $\begin{array}{l}\text { GIA: } 242 \text { r } 244+\text { VA: } 172 \text { c EMA: } 172+428+ \\
\text { 429c } 431+\text { LMA: } 427 \text { c } 428+430+\end{array}$ & \\
\hline Apium graveolens $\mathbf{L}$. & $\begin{array}{l}\text { RIA: } 311+\text { VA: 304r 307c 334c EMA: 521ru } \\
\text { LMA: } 521 \text { ru }\end{array}$ & $\begin{array}{l}\text { BI: VIIb-VIII (Godwin 1975), Late 2nd } \\
\text { century (Wilson 1979) NL: 600-400 B.C. } \\
\text { (Zeist 1974) BRD: 12-8 B.C. (Kučan 1981), } \\
\text { 1st century A.D. (Knörzer 1970), c. } 200 \\
\text { A.D. (Körber-Grohne 1979b) DDR: } \\
\text { Migration Period (Lange 1979) }\end{array}$ \\
\hline Arctium minus Bernh. & VA: $304 c$ & BRD: 1st century A.D. (Knörzer 1970) \\
\hline Arctium cf. minus Bernh. & VA: $334 c$ & \\
\hline
\end{tabular}




\begin{tabular}{|c|c|c|}
\hline Species & $\begin{array}{l}\text { Period, site number (cf. Table 1) and number } \\
\text { of macrofossils reported }\end{array}$ & $\begin{array}{l}\text { Reports on finds from neighbour countries. } \\
\text { Pollen zones and other periods are presented } \\
\text { as published by the authors cited }\end{array}$ \\
\hline Arctium sp. & $\begin{array}{l}\text { VI, VII: 555r GIA: 242r VA: 307r EMA: } \\
245+\text { 428r 521ru LMA: } 521 \text { ru }\end{array}$ & $\begin{array}{l}\text { Arctium cf. lappa L. recorded from NL: } \\
\text { 3400-3300 B.C. (Zeist \& Palfenier-Vegter } \\
\text { 1981) }\end{array}$ \\
\hline $\begin{array}{l}\text { Arctostaphylos alpinus (L.) } \\
\text { Sprengel (A. alpina) }\end{array}$ & II: $155 \mathrm{r}$ III: $416 \mathrm{ru} 417 \mathrm{ru} 506+540+995 \mathrm{r}$ & \\
\hline $\begin{array}{l}\text { Arctostaphylos uva-ursi (L.) } \\
\text { Sprengel }\end{array}$ & I: $155 \mathrm{r}$ II: $104 \mathrm{r} 155+506+513 \mathrm{c} 523 \mathrm{r}$ & \\
\hline $\begin{array}{l}\text { Arctostaphylos cf. uva-ursi } \\
\text { (L.) Sprengel }\end{array}$ & II: $508 \mathrm{c}$ & \\
\hline Arctostaphylos sp. & II: 509r 554r III: 152ru 426ru & \\
\hline Arenaria serpyllifolia $\mathbf{L}$. & VIII: 405r VA: 985r EMA: 430+ LMA: 521ru & $\begin{array}{l}\text { BI: I, VIII (Godwin 1975) NL: } 1155 \pm 65 \\
\text { B.C. (Zeist 1968) }\end{array}$ \\
\hline $\begin{array}{l}\text { Armeria maritima (Miller) } \\
\text { Willd. (A. vulgaris Willd., } \\
\text { Statice armeria L.) }\end{array}$ & $\begin{array}{l}\text { III: } 152+\text { u 161ru 415ru 417ru 507ru 720r VA: } \\
307 \mathrm{c}\end{array}$ & BI: I-IV, VI, VIIb (Godwin 1975) \\
\hline $\begin{array}{l}\text { Arnoseris minima (L.) } \\
\text { Schweigger \& Koerte }\end{array}$ & EMA: 521ru & \\
\hline Artemisia campestris L. & GIA: $712 \mathrm{r}$ & \\
\hline Artemisia vulgaris $\mathrm{L}$. & VA: $985 r$ & $\begin{array}{l}\text { NL: } 3400-3300 \text { B.C. (Zeist \& Palfenier- } \\
\text { Vegter 1981) BRD: } 12-8 \text { B.C. (Kučan 1981) } \\
\text { N: Late Viking (Tallantire 1979) }\end{array}$ \\
\hline Aster tripolium $\mathbf{L}$. & $\begin{array}{l}\text { RIA: } 311 \mathrm{c} \text { VA: } 304 \mathrm{r} 307 \mathrm{c} 334+\text { EMA: } 428+ \\
\text { 522c }\end{array}$ & $\begin{array}{l}\text { BI: IV/V, VIIa (Godwin 1975) NL: 3400- } \\
\text { 3300 B.C. (Zeist \& Palfenier-Vegter 1981) } \\
\text { BRD: 1st-2nd century A.D. (Körber- } \\
\text { Grohne 1967) }\end{array}$ \\
\hline Atriplex hastata $\mathbf{L}$. & RIA: 306c GIA: 306r VA: 334c & $\begin{array}{l}\text { BI: II, VI-VIII (Godwin 1975), 100-300 } \\
\text { A.D. (Wilson 1968) BRD: 12-8 B.C. } \\
\text { (Kučan 1981) }\end{array}$ \\
\hline Atriplex cf. hastata $\mathrm{L}$. & $\begin{array}{l}\text { PRIA: 301r RIA: 301c 315+ GIA: 301r VA: } \\
\text { 301+ 304r 320c }\end{array}$ & \\
\hline $\begin{array}{l}\text { Atriplex hastata L. et A. } \\
\text { littoralis L. }\end{array}$ & RIA: 311c VA: 307c & \\
\hline $\begin{array}{l}\text { Atriplex hastata L. (A. } \\
\text { deltoides Bab.), A. littoralis } \\
\text { L. et A. patula L. }\end{array}$ & VA: $202 \mathrm{r}$ & \\
\hline Atriplex littoralis $\mathrm{L}$. & VI, VII: $529+\mathrm{u}$ & \\
\hline Atriplex patula L. & $\begin{array}{l}\text { VI, VII: } 520+u \text { 528+u RIA: } 139 \text { r } 306+\text { GIA: } \\
306+712+\text { VA: } 312 \text { c 334c } 985+\end{array}$ & $\begin{array}{l}\text { BI: Late Weichselian, Early Flandrian, } \\
\text { VIIb-VIII (Godwin 1975) }\end{array}$ \\
\hline Atriplex cf. patula L. & $\begin{array}{l}\text { PRIA: 301r RIA: 301c 308+311c 315+ GIA: } \\
\text { 301+ VA: 301c 304c 307c 320c }\end{array}$ & \\
\hline Atriplex sp. & $\begin{array}{l}\text { V: 104r VI, VII: 159r PRIA: 301+ RIA: } \\
\text { 301+ GIA: 243+ VA: 103+ 172r 301r EMA: } \\
\text { 172+ 245c } 429+431+521 \text { ru LMA: 245c } 429+\end{array}$ & \\
\hline Avena fatua $\mathrm{L}$. & $\begin{array}{l}\text { VIII: 968r 970r 1032r PRIA: } 115+\text { RIA: } 107 r \\
\text { 108+ 115+ 139r 215r 309r 806r 1087r GIA: } \\
\text { 133r 713r VA: } 334 \text { r 1080r 1097r }\end{array}$ & $\begin{array}{l}\text { P: Neolithic, Bronze Age (Gluza \& } \\
\text { Wasylikowa 1977) }\end{array}$ \\
\hline Avena cf. fatua $\mathrm{L}$. & RIA: $106 r$ & \\
\hline $\begin{array}{l}\text { Avena fatua L. et A. sativa } \\
\text { L. }\end{array}$ & $\begin{array}{l}\text { VIII: 1055r RIA: 707+ 708+ 1087r GIA: } \\
\text { 712+ 713+ 1087r VA: 1087r EMA: 709c }\end{array}$ & \\
\hline Avena sativa $\mathrm{L}$. & $\begin{array}{l}\text { VIII: 1092r PRIA: } 115+\text { RIA: } 107 \mathrm{c} 108+ \\
\text { 309+ GIA: 205c VA: } 103 \mathrm{c} 301+304 \mathrm{c} 307 \mathrm{c} \\
\text { 312c 320c 334c 985c EMA: } 992+\text { LMA: } 218 \mathrm{c} \\
\text { 406r 522+957c }\end{array}$ & P: Bronze Age (Gluza \& Wasylikowa 1977) \\
\hline
\end{tabular}




\begin{tabular}{|c|c|c|}
\hline Species & $\begin{array}{l}\text { Period, site number (cf. Table 1) and number } \\
\text { of macrofossils reported }\end{array}$ & $\begin{array}{l}\text { Reports on finds from neighbour countries. } \\
\text { Pollen zones and other periods are presented } \\
\text { as published by the authors cited }\end{array}$ \\
\hline Avena strigosa Schreber & VIII: 1047 r GIA: 301r VA: 301+ & \\
\hline Avena cf. strigosa Schreber & VIII: $1045 \mathrm{r} 1046 \mathrm{r}$ & \\
\hline Avena sp. & 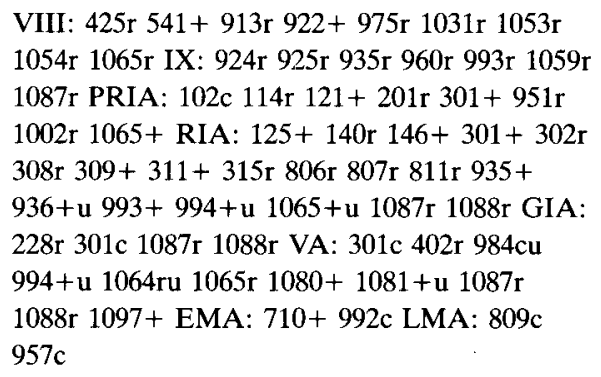 & \\
\hline Barbarea sp. & GIA: $244 c$ & $\begin{array}{l}\text { BI: II, Roman Period (Godwin 1975) BRD: } \\
\text { 1st century A.D. (Knörzer 1970) }\end{array}$ \\
\hline $\begin{array}{l}\text { Betula intermedia (according } \\
\text { to Hartz (1902) a cross } \\
\text { between B. nana and B. } \\
\text { pubescens or B. pendula) }\end{array}$ & II: 510c 511c IV: 238ru V: 238ru & \\
\hline Betula nana $\mathrm{L}$. & 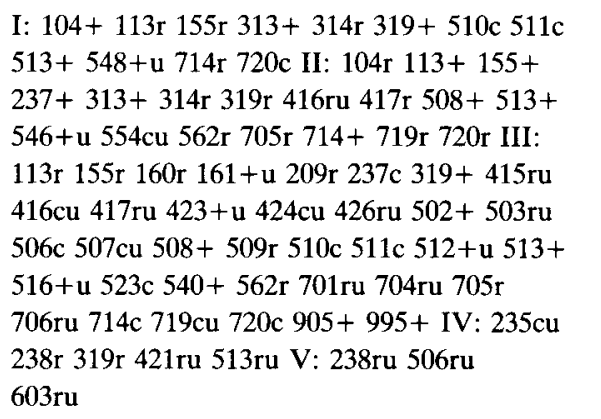 & BI: I-VIIb (Godwin 1975) \\
\hline Betula cf. nana $L$. & II: $547 \mathrm{ru} 706 \mathrm{ru}$ & \\
\hline $\begin{array}{l}\text { Betula nana L. } \times \text { B. } \\
\text { pubescens Ehrh. }\end{array}$ & II: $502+508 \mathrm{r}$ & BI: Late Weichselian, IV-V (Godwin 1975) \\
\hline $\begin{array}{l}\text { Betula pendula Roth (B. } \\
\text { verrucosa Ehrh.) }\end{array}$ & $\begin{array}{l}\text { II: } 510+511+\text { III: } 104 \text { r IV: } 209 \mathrm{c} \text { VI, VII: } \\
\text { 513r } 603 \text { ru } 159 \mathrm{c} 164+508 \mathrm{cu} 513+\mathrm{u} 603 \mathrm{ru} \\
\text { VIII: 323r } 328+513+\mathrm{u} \text { IX: } 332+513+\text { GIA: } \\
\text { 244+ VA: } 304 \mathrm{r} \text { 334r EMA: } 245+428+\text { LMA: } \\
241+428 \mathrm{r} 521+\mathrm{u}\end{array}$ & BI: I-VIII (Godwin 1975) \\
\hline $\begin{array}{l}\text { Betula pendula Roth } \times \text { B. } \\
\text { pubescens Ehrh. }\end{array}$ & IV: 711r V: 711ru IX: 711r & \\
\hline $\begin{array}{l}\text { Betula pendula Roth et B. } \\
\text { pubescens Ehrh. (B. alba L.) }\end{array}$ & $\begin{array}{l}\text { II: } 416+u \text { 417ru 509r } 513+546 r \text { 720r III: } \\
\text { 419ru 523r IV: 421ru 905r 1036r V: 549r 603ru } \\
905+1036 r \text { VI, VII: } 549 r \text { } 905+944 r \text { 1036r } \\
905+944 r \text { 1036r VIII: } 508+559 r \text { } 905+944 r \\
1036 r \text { IX: } 508+559 r \text { r } 905+944 r \text { r } 1036 r\end{array}$ & \\
\hline Betula pubescens Ehrh. & $\begin{array}{l}\text { I: } 313 \text { r } 314+319+\text { II: } 104+237 \mathrm{c} 313+314+ \\
319+410 \mathrm{r} 419 \mathrm{c} 508 \mathrm{c} 513+\mathrm{u} 523 \mathrm{c} 538+827 \mathrm{r} \\
\text { III: } 104+161 \text { ru } 237+319+\text { IV: } 209 \mathrm{c} 319 \mathrm{c} \\
\text { 502+ 508cu 711r V: } 502+506+\mathrm{u} 508 \mathrm{cu} 549 \mathrm{r} \\
603 \mathrm{ru} 711 \mathrm{ru} \text { VI, VII: } 410 \mathrm{r} 501+\mathrm{u} 502+\mathrm{u} 508 \mathrm{c} \\
\text { 603r 711r } 159+164+410 \mathrm{r} 501 \mathrm{c} 502+508 \mathrm{cu} \\
\text { 513cu 520+u 553ru 603ru VIII: } 220+410 \mathrm{r} \\
\text { 501c 502+ 513cu 603ru IX: 329r 501c 502+ } \\
\text { 513c 711r RIA: } 101+311+\text { VA: } 304+307+ \\
\text { 334+ EMA: 521ru LMA: } 427+\end{array}$ & BI: I-VIII (Godwin 1975) \\
\hline Betula cf. pubescens Ehrh. & V: 539ru LMA: $428 \mathrm{r}$ & \\
\hline
\end{tabular}




\begin{tabular}{|c|c|c|}
\hline Species & $\begin{array}{l}\text { Period, site number (cf. Table 1) and number } \\
\text { of macrofossils reported }\end{array}$ & $\begin{array}{l}\text { Reports on finds from neighbour countries. } \\
\text { Pollen zones and other periods are presented } \\
\text { as published by the authors cited }\end{array}$ \\
\hline $\begin{array}{l}\text { Betula pubescens Ehrh. } \\
\text { subsp. carpatica (Willd.) } \\
\text { Ascherson \& Graebner (B. } \\
\text { odorata Bechst) }\end{array}$ & $\begin{array}{l}\text { I: } 510 \text { r } 511 \text { r II: } 510+511+547+\text { u 562r III: } \\
\text { 704ru IV: } 238 \text { ru V: } 238 \text { ru 555r VI, VII: } 512+\text { u } \\
\text { 422cu LMA: } 217+u\end{array}$ & \\
\hline $\begin{array}{l}\text { Betula cf. pubescens Ehrh. } \\
\text { subsp. carpatica (Willd.) } \\
\text { Ascherson \& Graebner }\end{array}$ & VI, VII: 235ru VIII: 235ru LMA: 428r & \\
\hline Betula sp. & $\begin{array}{l}\text { II: } 113 \text { r III: 330r IV: } 330 \mathrm{c} \text { VI, VII: } 322+ \\
\text { 322+ 906c VIII: } 213 \text { ru } 323+328+329 r \text { r } 906+ \\
\text { IX: 327r 328+ 329r 906c RIA: } 311+\text { VA: } \\
\text { 304+ 307+334c }\end{array}$ & \\
\hline $\begin{array}{l}\text { Bidens cernua L. (B. } \\
\text { cernuus L.) }\end{array}$ & $\begin{array}{l}\text { VI, VII: } 422 \mathrm{cu} \text { IX: } 501 \mathrm{c} \text { RIA: } 101 \mathrm{c} \text { VA: } 334 \mathrm{c} \\
\text { LMA: } 218 \mathrm{r} 521+\mathrm{u}\end{array}$ & $\begin{array}{l}\text { BI: Late Weichselian, VIIb-VIII (Godwin } \\
\text { 1975) }\end{array}$ \\
\hline Bidens tripartita L. & $\begin{array}{l}\text { RIA: } 301+311 \mathrm{c} \text { VA: } 307 \mathrm{r} 334+\text { EMA: } 521 \mathrm{ru} \\
\text { LMA: } 521 \mathrm{ru} 985 \mathrm{r}\end{array}$ & $\begin{array}{l}\text { BI: VIIb/VIII (Godwin 1975), } 3360 \pm 80 \\
\text { B.P. (Peglar \& Wilson 1978), Iron Age } \\
\text { (Greig 1979a) NL: 1000 B.C. (Pals 1977), } \\
2860 \pm 30 \text { B.P. (Pals et al. 1980) BRD: 12-8 } \\
\text { B.C. (Kučan 1981), 1st-2nd century A.D. } \\
\text { (Körber-Grohne 1967, 1979b) }\end{array}$ \\
\hline $\begin{array}{l}\text { Bilderdykia convolvulus (L.) } \\
\text { Dumort (Polygonum } \\
\text { convolvulus L.) }\end{array}$ & $\begin{array}{l}\text { VIII } 127+132 \mathrm{r} 214 \mathrm{c} 411 \mathrm{r} 524 \mathrm{r} 922 \mathrm{r} 1089 \mathrm{r} \\
\text { PRIA: 109r 111c } 114+115+141+148+201 \mathrm{r} \\
\text { 301+ RIA: 107+ 108c } 139+140 \mathrm{r} 301 \mathrm{c} 302 \mathrm{r} \\
\text { 308c 708c GIA: } 133 \mathrm{r} \text { 135r } 205 \mathrm{r} 242+301 \mathrm{r} 306 \mathrm{r} \\
\text { 712c } 713+\text { VA: } 103+110+172 \mathrm{r} 301+304 \mathrm{c} \\
\text { 307+ 312r 334c } 985+\text { EMA: } 172 \mathrm{r} 245+427+ \\
428+429+521 \mathrm{cu} 992+\text { LMA: } 218+241+ \\
406 \mathrm{r} 427+428 \mathrm{r} 521+\mathrm{u} 522+809 \mathrm{r}\end{array}$ & $\begin{array}{l}\text { BI: VIIa-VIII (Godwin 1975) NL: } 1370 \pm 60 \\
\text { B.C. (Zeist 1968) BRD: Band Ceramic } \\
\text { (Knörzer 1974a, 1977) P: Neolithic, Bronze } \\
\text { Age (Gluza \& Wasylikowa 1977) }\end{array}$ \\
\hline $\begin{array}{l}\text { Blysmus compressus (L.) } \\
\text { Panzer ex Link (Scirpus } \\
\text { compressus (L.) Pers., non } \\
\text { Moench) }\end{array}$ & EMA: 521ru LMA: $521+u$ & BI: II (Godwin 1975) \\
\hline $\begin{array}{l}\text { Blysmus rufus (Hudson) } \\
\text { Link (Scirpus rufus (Hudson) } \\
\text { Schrader) }\end{array}$ & EMA: 521ru & $\begin{array}{l}\text { BI: VIIa (Godwin 1975) N: Late Viking } \\
\text { (Tallantire 1979) }\end{array}$ \\
\hline $\begin{array}{l}\text { Brachypodium sylvaticum } \\
\text { (Hudson) Beauv. }\end{array}$ & VA: $110 \mathrm{r}$ & \\
\hline Brassica cf. napus L. & LMA: $522+$ & $\begin{array}{l}\text { BI: Roman Period (Godwin 1975). Brassica } \\
\text { napus recorded from P: 13th century A.D. } \\
\text { (Wieserowa 1979) }\end{array}$ \\
\hline $\begin{array}{l}\text { Brassica napus L. et B. rapa } \\
\text { L. (B. campestris L.) }\end{array}$ & VA: $304 \mathrm{c}$ & \\
\hline $\begin{array}{l}\text { Brassica nigra (L.) Koch in } \\
\text { Röhling }\end{array}$ & EMA: $521+u$ LMA: $521+u$ & $\begin{array}{l}\text { BI: Roman Period (Godwin 1975) BRD: } \\
\text { Early Middle Ages (Lynch \& Paap 1982) } \\
\text { DDR: c. 2nd-1st century B.C. (Lange 1975) } \\
\text { P: 11th-12th century A.D. (Wasylikowa } \\
\text { 1978), Early Medieval Period (Gluza \& } \\
\text { Wasylikowa 1977) }\end{array}$ \\
\hline $\begin{array}{l}\text { Brassica cf. nigra (L.) Koch } \\
\text { in Röhling }\end{array}$ & $\begin{array}{l}\text { VIII: } 425+\text { PRIA: 301r RIA: } 301+\text { GIA: 301r } \\
\text { VA: } 301 \mathrm{c}\end{array}$ & \\
\hline Brassica oleracea $\mathrm{L}$. & VA: $110 \mathrm{r}$ & \\
\hline $\begin{array}{l}\text { Brassica rapa L. (B. } \\
\text { campestris L.) }\end{array}$ & $\begin{array}{l}\text { VIII: } 127 \text { r } 702+\text { PRIA: } 109+114+\text { RIA: } \\
\text { 107+ 108+ 139r 311c GIA: 228r VA: 202r } \\
\text { 307c 320c 334c EMA: } 428+521+\text { u LMA: } \\
\text { 171r } 241+521 \text { ru }\end{array}$ & BI: VIIb-VIII (Godwin 1975) \\
\hline $\begin{array}{l}\text { Brassica cf. rapa L. (B. } \\
\text { campestris L.) }\end{array}$ & LMA: $406 c$ & \\
\hline
\end{tabular}




\begin{tabular}{|c|c|c|}
\hline Species & $\begin{array}{l}\text { Period, site number (cf. Table 1) and number } \\
\text { of macrofossils reported }\end{array}$ & $\begin{array}{l}\text { Reports on finds from neighbour countries. } \\
\text { Pollen zones and other periods are presented } \\
\text { as published by the authors cited }\end{array}$ \\
\hline Brassica sp. & $\begin{array}{l}\text { IX: 935r RIA: 935r GIA: 242r 244+ EMA: } \\
\text { 521ru 172r 428r LMA: } 241+427+\end{array}$ & \\
\hline $\begin{array}{l}\text { Bromus hordeaceus } L \text {. } \\
\text { subsp. hordeaceus (B. mollis } \\
\text { L.) }\end{array}$ & $\begin{array}{l}\text { VIII: 974r PRIA: 115c RIA: 311c VA: } 307 \mathrm{c} \\
\text { 985r EMA: 992r }\end{array}$ & \\
\hline $\begin{array}{l}\text { Bromus cf. hordeaceus L. } \\
\text { subsp. hordeaceus }\end{array}$ & $\begin{array}{l}\text { VIII: } 1034 \text { r PRIA: 301r RIA: 301r GIA: 301r } \\
\text { VA: 301r 334+ }\end{array}$ & \\
\hline $\begin{array}{l}\text { Bromus hordeaceus } \mathrm{L} \text {. } \\
\text { subsp. hordeaceus et } \mathrm{B} \text {. } \\
\text { secalinus } \mathrm{L} \text {. }\end{array}$ & VIII: $116 \mathrm{c}$ & \\
\hline Bromus racemosus L. & RIA: 306r & $\begin{array}{l}\text { BRD: 1st century A.D. (Knörzer } \\
\text { 1970) }\end{array}$ \\
\hline Bromus secalinus L. & $\begin{array}{l}\text { VIII: 409r 609c } 819+909 \text { c } 914 \text { r } 946 r \text { 958+ } \\
\text { 959+962r 965+968r 971r 972r 974+976r } \\
\text { 979r 990r 1003r 1006r 1032+1034+1049+ } \\
\text { 1050+ 1074r 1086r 1089+ IX: 935r RIA: } \\
\text { 805ru 935+ 993r VA: 202c EMA: } 992+\end{array}$ & $\begin{array}{l}\text { BI: VIIb-VIII (Godwin 1975) BRD: Band } \\
\text { Ceramic (Knörzer 1974a, 1977) P: Neolithic, } \\
\text { Bronze Age (Gluza \& Wasylikowa 1977) }\end{array}$ \\
\hline Bromus cf. secalinus $\mathbf{L}$. & $\begin{array}{l}\text { VIII: } 129 \text { r 425c RIA: } 301+\text { GIA: } 242 \text { r 301r } \\
\text { VA: 301c 312r 334c EMA: } 428+\end{array}$ & \\
\hline Bromus sp. & $\begin{array}{l}\text { VIII: } 208+910 \mathrm{c} 922+966+975 \mathrm{r} 978+1005 \mathrm{r} \\
1031+1051+1069+\text { IX: } 993 \mathrm{r} \text { PRIA: } 301 \mathrm{r} \\
\text { RIA: } 139+301+308 \mathrm{r} 315+936+\mathrm{u} 994 \mathrm{ru} \\
\text { GIA: } 301 \mathrm{r} 712+\text { VA: } 301 \mathrm{r} 304 \mathrm{c}\end{array}$ & \\
\hline Bupleurum rotundifolium $\mathrm{L}$. & EMA: $245+$ & $\begin{array}{l}\text { BRD: 12-8 B.C. (Kučan 1981) DDR: 6th- } \\
\text { 10th century A.D. (Lange 1979), 7th-9th } \\
\text { century (Lange 1976) P: 9th-12th century } \\
\text { A.D. (Wasylikowa 1978) }\end{array}$ \\
\hline Bupleurum tenuissimum L. & EMA: 521ru & $\begin{array}{l}\text { BI: 100-300 A.D. (Wilson 1968), Roman } \\
\text { Period (Godwin 1975) }\end{array}$ \\
\hline Butomus umbellatus L. & LMA: $521+\mathrm{u}$ & BI: VIIb (Godwin 1975) \\
\hline Cakile maritima Scop. & VA: $103 r$ & \\
\hline Calla palustris $\mathrm{L}$. & $\begin{array}{l}\text { VI, VII: } 501+\text { VIII: } 501+\text { IX: 501c 502+ } \\
\text { RIA: } 101+\end{array}$ & \\
\hline $\begin{array}{l}\text { Callitriche hermaphroditica } \\
\text { L. (C. autumnalis L.) }\end{array}$ & I: $510 \mathrm{c} 511 \mathrm{c} 548 \mathrm{cu}$ III: $426 \mathrm{ru} 507+\mathrm{u}$ & \\
\hline Callitriche stagnalis Scop. & RIA: $101 \mathrm{r}$ & BI: II-III (Godwin 1975) \\
\hline Callitriche sp. & RIA: $301+$ LMA: $521+u$ & \\
\hline Calluna vulgaris (L.) Hull & $\begin{array}{l}\text { III: } 161+\text { u 415ru 704ru 705r IV: } 175 \mathrm{c} \text { V: } 175 \mathrm{c} \\
\text { VI, VII: } 175 \mathrm{c} 175 \mathrm{c} 322 \mathrm{r} 501 \mathrm{c} 944 \mathrm{r} \text { VIII: } 127 \mathrm{r} \\
207 \mathrm{c} 219 \mathrm{c} 229 \mathrm{c} 230 \mathrm{c} 501 \mathrm{c} 944 \mathrm{r} \text { IX: 167r 331r } \\
332+501 \mathrm{c} 502+508+944 \mathrm{r} \text { PRIA: } 301+\text { RIA: } \\
101+107 \mathrm{c} 128+301 \mathrm{c} 311 \mathrm{c} \text { GIA: } 135 \mathrm{r} 243+ \\
301+\text { VA: } 103+172+301 \mathrm{c} 304 \mathrm{c} 307 \mathrm{c} 34 \mathrm{c} \\
\text { EMA: } 172+\text { LMA: } 218+522 \mathrm{r}\end{array}$ & BI: III-VIII (Godwin 1975) \\
\hline Caltha palustris $\mathrm{L}$. & II: 155 r III: 506ru VIII: 531r VA: 202r 543r & BI: I-VI, VIIb-VIII (Godwin 1975) \\
\hline $\begin{array}{l}\text { Calystegia sepium (L.) R. } \\
\text { Br. (Convolvulus sepium L.) }\end{array}$ & VIII: $531 \mathrm{r}$ & \\
\hline $\begin{array}{l}\text { Camelina alyssum (Miller) } \\
\text { Thell. (C. linicola Schimper } \\
\text { \& Spenner) }\end{array}$ & $\begin{array}{l}\text { VIII: 127r PRIA: } 111+114 \mathrm{c} 115 \mathrm{r} \text { 119r 123r } \\
\text { 201c 211r RIA: 107c 108c 117r 118r 125c 126r } \\
\text { 139+ 151c 707r 708r GIA: 122r EMA: 709c } \\
\text { 710c }\end{array}$ & \\
\hline
\end{tabular}


Table 2 continued

\begin{tabular}{|c|c|c|}
\hline Species & $\begin{array}{l}\text { Period, site number (cf. Table 1) and number } \\
\text { of macrofossils reported }\end{array}$ & $\begin{array}{l}\text { Reports on finds from neighbour countries. } \\
\text { Pollen zones and other periods are presented } \\
\text { as published by the authors cited }\end{array}$ \\
\hline Camelina sativa (L.) Crantz & $\begin{array}{l}\text { IX: 935c 993r PRIA: } 148+\text { RIA: 301c 303+ } \\
\text { 308c 311c 316c 806r 935c 936cu 993r 994ru } \\
1065+\text { VA: } 307+320+334 c 1080+1081+u \\
1097+\end{array}$ & $\begin{array}{l}\text { BRD: Hallstatt (Knörzer 1971c, 1974b), c. } \\
200 \text { B.C. (Knörzer 1979a) DDR: Late La } \\
\text { Têne (Lange 1975) P: Hallstatt, La Tène } \\
\text { (Gluza \& Wasylikova 1977) S: Late Bronze } \\
\text { Age (Hjelmqvist 1979) }\end{array}$ \\
\hline Camelina sp. & PRIA: 141c RIA: $140+$ GIA: $1087+$ & \\
\hline Campanula glomerata $\mathbf{L}$. & PRIA: $115 r$ & \\
\hline Campanula rapunculoides L. & VA: $304+334+$ & $\begin{array}{l}\text { P: Hallstatt (Gluza \& Wasylikowa } \\
\text { 1977) }\end{array}$ \\
\hline $\begin{array}{l}\text { Campanula rotundifolia } \mathrm{L} \text {. et } \\
\text { Jasione montana } \mathrm{L} \text {. }\end{array}$ & VA: $304 \mathrm{c} 334 \mathrm{c}$ & \\
\hline $\begin{array}{l}\text { Capsella bursa-pastoris (L.) } \\
\text { Medicus }\end{array}$ & $\begin{array}{l}\text { VIII: } 609+\text { PRIA: } 111+114 \text { r } 115+\text { RIA: } 139 \text { r } \\
\text { 708r GIA: 712r LMA: } 427+\end{array}$ & $\begin{array}{l}\text { NL: } 3400-3300 \text { B.C. (Zeist \& Palfenier- } \\
\text { Vegter 1981) }\end{array}$ \\
\hline $\begin{array}{l}\text { Capsella cf. bursa-pastoris } \\
\text { (L.) Medicus }\end{array}$ & RIA: $301 \mathrm{c}$ & \\
\hline Carduus crispus $L$. & $\begin{array}{l}\text { GIA: } 244+\text { VA: } 110+334 \mathrm{c} \text { EMA: } 521 \mathrm{cu} \\
\text { LMA: } 521+\text { u } 1025 \mathrm{r}\end{array}$ & $\begin{array}{l}\text { NL: } 3400-3300 \text { B.C. (Zeist \& Palfenier- } \\
\text { Vegter 1981) BRD: 1st century A.D. } \\
\text { (Knörzer 1970) }\end{array}$ \\
\hline Carduus cf. crispus $\mathrm{L}$. & VA: $985 r$ & \\
\hline Carduus nutans $\mathbf{L}$. & VA: $334 \mathrm{r}$ & $\begin{array}{l}\text { BI: } 2130 \pm 100 \text { B.P. (Greig 1979b), Roman } \\
\text { Period (Godwin 1975), 100-300 A.D. } \\
\text { (Wilson 1968) }\end{array}$ \\
\hline Carduus sp. & LMA: $430+$ & \\
\hline Carex acuta L. & IX: 553ru GIA: 553+ VA: 304+ 334+ & $\begin{array}{l}\text { Carex acuta-type recorded from NL: } 200 \\
\text { B.C.-250 A.D. (Zeist 1974) }\end{array}$ \\
\hline Carex cf. acuta L. & VIII: 603ru IX: $513 \mathrm{c}$ & \\
\hline $\begin{array}{l}\text { Carex appropinquata } \\
\text { Schumacher }\end{array}$ & VA: $334 \mathrm{c}$ & BI: VI, Roman Period (Godwin 1975) \\
\hline $\begin{array}{l}\text { Carex cf. appropinquata } \\
\text { Schumacher }\end{array}$ & RIA: $101+$ & \\
\hline $\begin{array}{l}\text { Carex cf. aquatilis } \\
\text { Wahlenb. }\end{array}$ & III: $540 \mathrm{r}$ & \\
\hline Carex arenaria $\mathrm{L}$. & RIA: $139 \mathrm{c}$ & \\
\hline Carex cespitosa $\mathrm{L}$. & IX: $180+$ & \\
\hline $\begin{array}{l}\text { Carex curta Good. (C. } \\
\text { canescens auct. non L.) }\end{array}$ & $\begin{array}{l}\text { VI, VII: } 501+\text { VIII: } 501+\text { IX: } 501 \mathrm{c} 502+711 \mathrm{r} \\
\text { LMA: } 218+\end{array}$ & \\
\hline Carex cf. curta Good. & $\begin{array}{l}\text { IX: 329+ PRIA: 301r RIA: 301+ GIA: 301r } \\
\text { 712r VA: 301c }\end{array}$ & \\
\hline Carex diandra Schrank & IX: 513c LMA: 218r & $\begin{array}{l}\text { BI: VIII (Godwin 1975) BRD: c. } 1209 \text { A.D. } \\
\text { (Lynch \& Paap 1982) S: c. } 1300 \text { A.D. } \\
\text { (Griffin 1982) }\end{array}$ \\
\hline Carex cf. diandra Schrank & VIII: 603ru & \\
\hline Carex distans $\mathrm{L}$. & VA: $307 \mathrm{c}$ & \\
\hline Carex cf. distans $\mathrm{L}$. & RIA: 301c GIA: 301r VA: $301+$ & \\
\hline Carex disticha Hudson & $\begin{array}{l}\text { PRIA: 301+ RIA: 301c 311c GIA: 301r VA: } \\
\text { 301+ 307c LMA: } 218 \mathrm{c}\end{array}$ & $\begin{array}{l}\text { BI: IV (Godwin 1975) NL: } 3400-3300 \text { B.C. } \\
\text { (Zeist \& Palfenier-Vegter 1981) }\end{array}$ \\
\hline $\begin{array}{l}\text { Carex disticha Hudson et } \mathrm{C} \text {. } \\
\text { elongata } \mathrm{L} \text {. }\end{array}$ & VA: $304 c 334 c$ & \\
\hline
\end{tabular}


Table 2 continued

\begin{tabular}{|c|c|c|}
\hline Species & $\begin{array}{l}\text { Period, site number (cf. Table 1) and number } \\
\text { of macrofossils reported }\end{array}$ & $\begin{array}{l}\text { Reports on finds from neighbour countries. } \\
\text { Pollen zones and other periods are presented } \\
\text { as published by the authors cited }\end{array}$ \\
\hline Carex sect. Distigmaticae & $\begin{array}{l}\text { I: } 155 \text { c II: } 113 \mathrm{r} 155 \mathrm{c} \text { III: } 113+155 \mathrm{c} 540 \mathrm{r} \text { VIII: } \\
\text { 116+ GIA: } 242 \mathrm{c} 243 \mathrm{c} 244 \mathrm{c} \text { VA: } 172+202 \mathrm{c} \\
\text { EMA: } 172 \mathrm{c} 428 \mathrm{c} 429 \mathrm{c} 430+431 \mathrm{c} \text { LMA: } 241 \mathrm{c} \\
427 \mathrm{c} 428+430 \mathrm{c}\end{array}$ & \\
\hline $\begin{array}{l}\text { Carex echinata Murray (C. } \\
\text { stellulata Good.) }\end{array}$ & VA: 985r EMA: 172r LMA: 218c 406r & $\begin{array}{l}\text { BI: II-III/IV, VIIb/VIII (Godwin 1975) N: } \\
\text { Late Viking (Tallantire 1979) }\end{array}$ \\
\hline $\begin{array}{l}\text { Carex cf. echinata } \\
\text { Murray }\end{array}$ & VIII: $220 \mathrm{r}$ & \\
\hline $\begin{array}{l}\text { Carex elata All. (C. stricta } \\
\text { Good., non Lam.) }\end{array}$ & VIII: $328 \mathrm{c}$ VA: $304 \mathrm{c} 307 \mathrm{r}$ & BI: VIIb-VIII (Godwin 1975) \\
\hline Carex elongata L. & IX: $513+$ & BRD: IX ‘Overbeck’ (Schwaar 1976) \\
\hline Carex cf. elongata L. & IX: 329r & \\
\hline Carex extensa Good. & VA: $307 \mathrm{r}$ & BI: VII/VIII (Godwin 1975) \\
\hline Carex flacca Schreber & VA: $304+334+$ LMA: $809 \mathrm{r}$ & $\begin{array}{l}\text { BI: Late Weichselian, VI, Roman Period } \\
\text { (Godwin 1975), Late 2nd century A.D. } \\
\text { (Wilson 1979) BRD: c. } 200 \text { A.D. (Körber- } \\
\text { Grohne 1979b) }\end{array}$ \\
\hline Carex flava $\mathrm{L}$. & RIA: $311 \mathrm{r} 315 \mathrm{r}$ VA: $304 \mathrm{c} 334 \mathrm{c}$ & $\begin{array}{l}\text { Carex flava agg. recorded from BI: IV/V, } \\
\text { VIIa (Godwin 1975) BRD: 1st-2nd century } \\
\text { A.D. (Körber-Grohne 1967, 1979b, Knörzer } \\
\text { 1979b) }\end{array}$ \\
\hline Carex cf. flava $\mathbf{L}$. & $\begin{array}{l}\text { VIII: } 327+\text { PRIA: } 301+\text { RIA: } 301+708 \text { r } \\
\text { GIA: } 301+\text { VA: } 301+\end{array}$ & \\
\hline Carex hirta $\mathbf{L}$. & $\begin{array}{l}\text { PRIA: 301r RIA: } 301+308+\text { GIA: } 301 r \text { VA: } \\
\text { 301+ EMA: } 521+\text { u LMA: } 521 \mathrm{cu}\end{array}$ & \\
\hline $\begin{array}{l}\text { Carex lasiocarpa Ehrh. (C. } \\
\text { filiformis L.) }\end{array}$ & $\begin{array}{l}\text { IV: } 239 r \text { V: } 1090 \mathrm{c} \text { VI, VII: } 501+\text { u 513ru 549r } \\
\text { 944r } 177+422 \text { ru 501+ 502+513+u 944r VIII: } \\
501+502+513+u \text { 944r IX: } 513+944 \text { r LMA: } \\
218+\end{array}$ & \\
\hline Carex lepidocarpa Tausch & GlA: $205 \mathrm{r}$ & $\begin{array}{l}\text { BI: II, Roman Period (Godwin 1975), 100- } \\
300 \text { A.D. (Wilson 1968) }\end{array}$ \\
\hline Carex nigra (L.) Reichard & VA: 334c LMA: $809 \mathrm{r}$ & $\begin{array}{l}\text { BI: IV, VIIb (Godwin 1975) NL: 3400-3300 } \\
\text { B.C. (Zeist \& Palfenier-Vegter 1981) BRD: } \\
\text { 1st century A.D. (Knörzer 1970), 2nd } \\
\text { century A.D. (Knörzer 1979b) P: 9th-12th } \\
\text { century A.D. (Wasylikowa 1978) }\end{array}$ \\
\hline $\begin{array}{l}\text { Carex cf. nigra (L.) Reichard } \\
\text { (C. fusca-Typ) }\end{array}$ & PRIA: 301+ RIA: 301c GIA: 301+ VA: 301c & \\
\hline $\begin{array}{l}\text { Carex nigra (L.) Reichard et } \\
\text { C. rostrata Stokes in With. }\end{array}$ & VI, VII: 944r 944r VIII: 944r IX: $944+$ & \\
\hline $\begin{array}{l}\text { Carex ovalis Good. (C. } \\
\text { leporina L.) }\end{array}$ & PRIA: 115r EMA: $521+\mathrm{u}$ & BI: 12-8 B.C. (Kučan 1981) \\
\hline Carex panicea $L$. & VA: $304+$ & BI: II, III, VIIa-VIII (Godwin 1975) \\
\hline Carex cf. panicea $\mathbf{L}$. & & \\
\hline Carex cf. paniculata L. & IX: 711r & $\begin{array}{l}\text { Carex paniculata recorded from BI: III, V- } \\
\text { VIII (Godwin 1975) NL: } 3400-3300 \text { B.C. } \\
\text { (Zeist \& Palfenier-Vegter 1981) }\end{array}$ \\
\hline Carex pilulifera $\mathrm{L}$. & $\begin{array}{l}\text { VIII: } 207 \mathrm{c} 219 \mathrm{c} 229 \mathrm{c} 230 \mathrm{r} \text { RIA: } 301 \mathrm{c} \text { VA: } \\
301+334+\end{array}$ & \\
\hline
\end{tabular}


Table 2 continued

\begin{tabular}{|c|c|c|}
\hline Species & $\begin{array}{l}\text { Period, site number (cf. Table 1) and number } \\
\text { of macrofossils reported }\end{array}$ & $\begin{array}{l}\text { Reports on finds from neighbour countries. } \\
\text { Pollen zones and other periods are presented } \\
\text { as published by the authors cited }\end{array}$ \\
\hline Carex pseudocyperus L. & $\begin{array}{l}\text { IV: 209r 238ru V: 238ru 539ru VI, VII: } 501+\mathrm{u} \\
\text { 549c 555r 422cu 501+ VIII: 501+559ru 603ru } \\
\text { IX: } 502+513+\text { RIA: } 306+311 \mathrm{c} \text { GIA: } 135 \mathrm{r} \\
\text { VA: } 307 \mathrm{r} 334 \mathrm{c} \text { LMA: } 217 \mathrm{cu}\end{array}$ & \\
\hline Carex riparia Curtis & V: $711 \mathrm{cu}$ VA: $307+$ & BI: II-III, V-VI, VIII (Godwin 1975) \\
\hline Carex cf. riparia Curtis & $\begin{array}{l}\text { PRIA: 301r RIA: 301+ GIA: 301r VA: } \\
\text { 301+ }\end{array}$ & \\
\hline $\begin{array}{l}\text { Carex rostrata Stokes in } \\
\text { With. (C. ampullacea Good., } \\
\text { C. inflata sensu V. Krecz, } \\
\text { non Hudson) }\end{array}$ & $\begin{array}{l}\text { II: } 237 \mathrm{r} 416 \mathrm{cu} 417 \mathrm{r} 419 \mathrm{r} 508+509 \mathrm{r} 510 \mathrm{c} 511 \mathrm{c} \\
\text { 513+ 523c III: 104r 416ru 417ru 426ru 506+u } \\
\text { 510r 511r 513r 704ru IV: 209r 235cu V: 506ru } \\
\text { 711ru VI, VII: 501+u 711r 422ru IX: } 180+ \\
\text { 329r 501c 513c 711c RIA: } 101 \mathrm{r} \text { 128r GIA: } \\
\text { 306+ VA: 304c 334c LMA: } 217 \mathrm{cu} 218 \mathrm{c}\end{array}$ & $\begin{array}{l}\text { BI: I-VIII (Godwin 1975) NL: } 2510 \pm 35 \\
\text { B.P. (Geel et al. 1983) }\end{array}$ \\
\hline $\begin{array}{l}\text { Carex cf. rostrata Stokes in } \\
\text { With. }\end{array}$ & I: $155+$ II: 155 c III: $155+$ VIII: $220 \mathrm{r}$ & \\
\hline $\begin{array}{l}\text { Carex cf. spicata Hudson (C. } \\
\text { contigua Hoppe) }\end{array}$ & VA: $985 r$ & $\begin{array}{l}\text { BI: } 3360 \pm 80 \text { B.P. (Peglar \& Wilson 1978) } \\
\text { Carex spicata reported from: } 10580 \text { B.P. } \\
\text { (Godwin 1975) BRD: } 12-8 \text { B.C. (Kučan } \\
\text { 1981), 1st century A.D. (Knörzer 1970), and } \\
\text { from 2nd century A.D. (Knörzer 1979b) }\end{array}$ \\
\hline Carex strigosa Hudson & VA: $334+$ & BI: IV/V-VI, VIIb (Godwin 1975) \\
\hline Carex sect. Tristigmaticae & $\begin{array}{l}\text { II: } 113 \text { III: } 113+540 \text { r GIA: } 242+243+244 c \\
\text { VA: } 172 \text { c } 202 \text { c EMA: } 172+427+428 c 429 c \\
\text { 430c } 431+\text { LMA: } 241 c 427 c 429 c 430 c\end{array}$ & \\
\hline $\begin{array}{l}\text { Carex vesicaria L. (C. inflata } \\
\text { Hudson) }\end{array}$ & $\begin{array}{l}\text { II: 508r IV: 209r VIII: 559ru IX: 513c RIA: } \\
\text { 311+ EMA: 521+u LMA: 218+ 406r }\end{array}$ & \\
\hline Carex cf. vesicaria L. & IV: $711 \mathrm{r}$ & \\
\hline $\begin{array}{l}\text { Carex vulpina L. (incl. C. } \\
\text { otrubae Podp.) }\end{array}$ & RIA: $311 \mathrm{c}$ VA: $304 \mathrm{c} 307 \mathrm{c} 334 \mathrm{c}$ & $\begin{array}{l}\text { BI: VIII (Godwin 1975) NL: } 1000 \text { B.C. (Pals } \\
\text { 1977) BRD: 1st-2nd century A.D. (Körber- } \\
\text { Grohne 1967, 1979b) }\end{array}$ \\
\hline Carex cf. vulpina $\mathrm{L}$. & PRIA: 301r RIA: 301+ GIA: 301r VA: 301+ & \\
\hline Carex sp. & $\begin{array}{l}\text { I: } 314+513+\text { II: } 104+314+416 r u \text { 419c 546r } \\
\text { III: } 152 \text { ru 158+u 161+u 330c 415r 506cu } \\
\text { 507+u 509r 905r IV: 138ru 330c 905r V: 213r } \\
221 r \text { 506+u 905+ VI, VII: 213r 221r 322r 529r } \\
555+905+952+159+164 r \text { 175c } 905+906+ \\
\text { 944r VIII: 325r 326r 327c 328c 559r 905r 906r } \\
\text { 944r IX: 323+ 324+ 325r 326r 327c 328c 329c } \\
\text { 332+ 559r 905r 906r PRIA: 111+ 301+ RIA: } \\
\text { 101c 139r 301+ 306 + 311c 708r GIA: 135r } \\
\text { 242+ 301r 564+u VA: 301+ 304c EMA: } \\
\text { 521cu 564+ LMA: 521cu 522c 564+ }\end{array}$ & \\
\hline Carpinus betulus $\mathrm{L}$. & IX: $711 \mathbf{r}$ & $\begin{array}{l}\text { BI: VIIb-VIII (Godwin 1975) P: } \\
\text { Palaeolithic, Mesolithic, Neolithic (Gluza \& } \\
\text { Wasylikowa 1977) }\end{array}$ \\
\hline Centaurea cyanus $\mathbf{L}$. & $\begin{array}{l}\text { VA: } 334 \mathrm{r} \text { EMA: } 245+428+429+431+ \\
521+\text { u } 992+\text { LMA: } 218 \mathrm{c} 245+521 \mathrm{ru} 809 \mathrm{r}\end{array}$ & $\begin{array}{l}\text { BRD: 12-8 B.C. (Kučan 1981), c. 10th } \\
\text { century A.D. (Willerding 1973) DDR: 6th- } \\
\text { 10th century A.D. (Lange 1979), 7th-9th } \\
\text { century (Lange 1976) P: Neolithic, Hallstatt } \\
\text { and later (Gluza \& Wasylikowa 1977), 11th } \\
\text { century A.D. (Kosina 1978), 9th-12th } \\
\text { century (Wasylikowa 1978) }\end{array}$ \\
\hline Centaurea cf. cyanus L. & LMA: $427+$ & \\
\hline
\end{tabular}


Table 2 continued

\begin{tabular}{|c|c|c|}
\hline Species & $\begin{array}{l}\text { Period, site number (cf. Table 1) and number } \\
\text { of macrofossils reported }\end{array}$ & $\begin{array}{l}\text { Reports on finds from neighbour countries. } \\
\text { Pollen zones and other periods are presented } \\
\text { as published by the authors cited }\end{array}$ \\
\hline Centaurea jacea $\mathrm{L}$. & VA: 202 r LMA: $522 r$ & $\begin{array}{l}\text { BRD: 1st century A.D. (Knörzer 1967a), } \\
\text { 2nd century (Knörzer 1979b, Körber-Grohne } \\
\text { 1979b) P: Hallstatt and later (Gluza \& } \\
\text { Wasylikowa 1977), 9th-12th century A.D. } \\
\text { (Wasylikowa 1978) }\end{array}$ \\
\hline Centaurea scabiosa L. & VA: $110 \mathrm{c} 334 \mathrm{r}$ & $\begin{array}{l}\text { BI: II (Godwin 1975) P: 9th-12th century } \\
\text { A.D. (Wasylikowa 1978) }\end{array}$ \\
\hline Centaurea sp. & VA: 172r EMA: 521ru LMA: 521ru & \\
\hline Centaurium sp. & RIA: $311 \mathrm{c}$ VA: $304 \mathrm{r} 307 \mathrm{c} 334 \mathrm{c}$ & $\begin{array}{l}\text { BI: Late 3rd century B.C. (Wilson 1978) } \\
\text { NL: } 600-400 \text { B.C. (Zeist 1974) BRD: 1st- } \\
\text { 2nd century A.D. (Körber-Grohne 1967) }\end{array}$ \\
\hline $\begin{array}{l}\text { Cerastium fontanum Baumg. } \\
\text { subsp. triviale (Link) Jalas } \\
\text { (C. caespitosum Gilib., C. } \\
\text { holosteoides Fries) }\end{array}$ & $\begin{array}{l}\text { PRIA: 115r RIA: 139r GIA: } 244+712+\text { VA: } \\
\text { 202r 304r 307c 334c LMA: 171r } 427+\end{array}$ & BI: II-III, VIIb-VIII (Godwin 1975) \\
\hline Cerastium sp. & $\begin{array}{l}\text { RIA: } 301+\text { VA: 301r EMA: } 429+\text { LMA: } \\
521+\mathrm{u}\end{array}$ & \\
\hline Ceratophyllum demersum $\mathrm{L}$. & $\begin{array}{l}\text { III: } 104+\text { IV: 104r 238ru V: 238ru 603ru VI, } \\
\text { VII: 410r 555r 603ru 410r 422cu 513ru 603ru } \\
\text { VIII: 410r 603ru EMA: 430c LMA: } 217 \text { ru } \\
430+\end{array}$ & BI: I, IV-VIII (Godwin 1975) \\
\hline $\begin{array}{l}\text { Ceratophyllum submersum } \\
\text { L. }\end{array}$ & V: 104+ VI, VII: 603ru 603ru LMA: 521 ru & \\
\hline Ceratophyllum sp. & $\begin{array}{l}\text { V: } 944 r \text { VI, VII: } 944 r \text { } 826+944 \text { r 1036r VIII: } \\
\text { 1036r IX: } 1036 r\end{array}$ & \\
\hline Chelidonium majus $\mathbf{L}$. & LMA: $240 \mathrm{c} 430+$ & $\begin{array}{l}\text { BI: Roman, Medieval Period (Godwin 1975) } \\
\text { BRD: First centuries A.D. (Averdieck et al. } \\
\text { 1982), 819-1138 A.D. (Averdieck 1981) P: } \\
\text { 13th century A.D. (Wasylikowa 1978), Late } \\
\text { Medieval Period (Gluza \& Wasylikowa 1977) }\end{array}$ \\
\hline Chenopodium album $\mathrm{L}$. & $\begin{array}{l}\text { VI, VII: 321r 323r 520+u 528+u VIII: } 127 \mathrm{c} \\
\text { 207r 208c 219r 220c 230r 301c 322+ 323r 405+ } \\
\text { 411c 425c 543c 609c 965r 966r 974c IX: 323+ } \\
\text { 1087r PRIA: } 109+111 \mathrm{c} 114+115+121 \mathrm{r} 141 \mathrm{c} \\
\text { 148r 301c RIA: } 101+105 \mathrm{r} 107+108 \mathrm{c} 139 \mathrm{c} \\
\text { 301c 303c 306c 308c 309r 311c 708+ GIA: } \\
\text { 133r 135+ 205c 244c 301c 306c 712c 713+ VA: } \\
\text { 103c 110+ 202c 301c 304c 306r 307c 312c 334c } \\
\text { 985c EMA: 428c 429c 430c 431c 709r 992+ } \\
\text { LMA: } 217 \mathrm{cu} 218 \mathrm{c} 241 \mathrm{c} 406 \mathrm{c} 428 \mathrm{c} 429 \mathrm{c} 430+ \\
\text { 522c } 809+957 \mathrm{r} 1025+\end{array}$ & $\begin{array}{l}\text { BI: IV-VIII (Godwin 1975) NL: } 3400-3300 \\
\text { B.C. (Zeist \& Palfenier-Vegter 1981) }\end{array}$ \\
\hline Chenopodium cf. album L. & $\begin{array}{l}\text { RIA: } 128 \text { r GIA: } 242 \mathrm{c} 243+\text { VA: } 172 \mathrm{c} 427 \mathrm{c} \\
\text { EMA: } 172 \mathrm{c} 245 \mathrm{c} 427 \mathrm{c} \text { LMA: } 245 \mathrm{c} 427 \mathrm{c}\end{array}$ & \\
\hline Chenopodium ficifolium Sm. & $\begin{array}{l}\text { RIA: } 301+311 \mathrm{c} 315 \mathrm{r} \text { VA: } 301 \mathrm{r} 304+307 \mathrm{c} \\
320 \mathrm{c} 334 \mathrm{c}\end{array}$ & $\begin{array}{l}\text { BI: Iron Age (Greig 1979b), Late 2nd } \\
\text { century A.D. (Wilson 1979) NL: } 3230 \pm 35 \\
\text { B.P. (Pals et al. 1980) BRD: 12-8 B.C. } \\
\text { (Kučan 1981), 1st-2nd century A.D. } \\
\text { (Körber-Grohne 1967), 1st century A.D. } \\
\text { (Knörzer 1970) P: Hallstatt and later (Gluza } \\
\text { \& Wasylikowa 1977) }\end{array}$ \\
\hline Chenopodium cf. glaucum L. & VIII: $323+$ IX: $323+$ & \\
\hline $\begin{array}{l}\text { Chenopodium glaucum } \mathrm{L} \text {. et } \\
\text { C. rubrum } \mathrm{L} \text {. }\end{array}$ & PRIA: 301r RIA: 301c & \\
\hline
\end{tabular}


Table 2 continued

\begin{tabular}{|c|c|c|}
\hline Species & $\begin{array}{l}\text { Period, site number (cf. Table 1) and number } \\
\text { of macrofossils reported }\end{array}$ & $\begin{array}{l}\text { Reports on finds from neighbour countries. } \\
\text { Pollen zones and other periods are presented } \\
\text { as published by the authors cited }\end{array}$ \\
\hline Chenopodium murale L. & RIA: 306r 316c & $\begin{array}{l}\text { BI: Roman Period (Godwin 1975), 3rd } \\
\text { century A.D. (Willcox 1977) BRD: c. } 200 \\
\text { B.C. (Knörzer 1979a) }\end{array}$ \\
\hline $\begin{array}{l}\text { Chenopodium polyspermum } \\
\text { L. }\end{array}$ & RIA: $306 r$ & $\begin{array}{l}\text { BI: Roman Period (Godwin 1975), 1st-early } \\
\text { 2nd century A.D. (Kenward \& Williams } \\
\text { 1979), 100-300 A.D. (Wilson 1968) NL: } 500 \\
\text { B.C.-200 A.D. (Zeist 1974) BRD: 1st } \\
\text { century A.D. (Knörzer 1967a, 1970) DDR: } \\
\text { 1st century A.D. (Lange 1975) P: Neolithic } \\
\text { and later (Gluza \& Wasylikowa 1977) }\end{array}$ \\
\hline Chenopodium rubrum L. & RIA: 306c GIA: $306+$ & $\begin{array}{l}\text { BI: II, IV, VI-VIII (Godwin 1975), 4th-5th } \\
\text { century A.D. (Greig 1976) NL: Before } 1400 \\
\text { B.C. (Pals et al. 1980), } 1000 \text { B.C. (Pals } \\
\text { 1977) }\end{array}$ \\
\hline Chenopodium cf. rubrum $\mathrm{L}$. & RIA: 311r VA: 307c & \\
\hline Chenopodium cf. urbicum L. & VIII: $425 \mathrm{c}$ & $\begin{array}{l}\text { Chenopodium urbicum recorded from P: } \\
\text { Neolithic (Gluza \& Wasylikowa 1977) }\end{array}$ \\
\hline Chenopodium sp. & $\begin{array}{l}\text { VI, VII: 321r VIII: 542r IX: 557c PRIA: 115r } \\
\text { RIA: 146r GIA: 228r 243+ VA: 172+ EMA: } \\
\text { 428c 429c 431c 521cu LMA: } 241+427 \mathrm{c} 428 \mathrm{c} \\
429+521 \mathrm{cu}\end{array}$ & \\
\hline Chrysanthemum sp. & VA: $172 r$ & \\
\hline Cichorium intybus $\mathrm{L}$. & VA: 334 r EMA: $430+521+u$ & $\begin{array}{l}\text { BRD: 1st century A.D. (Knörzer 1970) P: } \\
\text { Early Medieval Period (Gluza \& Wasylikowa } \\
\text { 1977) }\end{array}$ \\
\hline Cicuta virosa $\mathrm{L}$. & $\begin{array}{l}\text { IV: 209r 995r VI, VII: 422ru 501+ VIII: } 501+ \\
\text { 559ru 603ru IX: } 329+502+513+\text { EMA: } \\
\text { 430+ LMA: } 217 \mathrm{cu}\end{array}$ & \\
\hline Cirsium arvense (L.) Scop. & $\begin{array}{l}\text { RIA: 306r GIA: } 306 \mathrm{r} \text { VA: } 110+985+\text { EMA: } \\
\text { 172+ 428r } 429+430+431+992 \text { r LMA: } 218+ \\
406 \mathrm{r} 427 \mathrm{c} 430+522 \mathrm{r}\end{array}$ & $\begin{array}{l}\text { BI: III, VIIb-VIII (Godwin 1975) NL: 3400- } \\
3300 \text { B.C. (Zeist \& Palfenier-Vegter 1981) } \\
\text { BRD: 12-8 B.C. (Kučan 1981), 1st century } \\
\text { A.D. (Knörzer 1970), c. } 200 \text { A.D. (Körber- } \\
\text { Grohne 1979b) }\end{array}$ \\
\hline $\begin{array}{l}\text { Cirsium cf. arvense (L.) } \\
\text { Scop. }\end{array}$ & VA: $307+$ EMA: $245+$ & \\
\hline $\begin{array}{l}\text { Cirsium arvense (L.) Scop. } \\
\text { et C. palustre (L.) Scop. }\end{array}$ & RIA: $301+$ & \\
\hline $\begin{array}{l}\text { Cirsium cf. helenioides (L.) } \\
\text { Hill (C. heterophyllum (L.) } \\
\text { Hill) }\end{array}$ & VA: $103 r$ & $\begin{array}{l}\text { Circium helenioides recorded from BI: I/II } \\
\text { (Godwin 1975) }\end{array}$ \\
\hline $\begin{array}{l}\text { Cirsium oleraceum (L.) } \\
\text { Scop. }\end{array}$ & VA: $202 r$ & $\begin{array}{l}\text { P: Hallstatt and later (Gluza \& Wasylikowa } \\
\text { 1977) }\end{array}$ \\
\hline $\begin{array}{l}\text { Cirsium oleraceum (L.) } \\
\text { Scop. et C. vulgare (Savi) } \\
\text { Ten. }\end{array}$ & VA: $202 \mathrm{r} 334 \mathrm{c}$ & \\
\hline Cirsium palustre (L.) Scop. & VI, VII: 528ru 555r VA: 110r LMA: $217+u$ & \\
\hline $\begin{array}{l}\text { Cirsium cf. palustre (L.) } \\
\text { Scop. }\end{array}$ & IX: 329r VA: 307r & \\
\hline $\begin{array}{l}\text { Cirsium vulgare (Savi) Ten. } \\
\text { (C. lanceolatum (L.) Scop., } \\
\text { non Hill) }\end{array}$ & VIII: 543r RIA: 301r LMA: $521+u$ & $\begin{array}{l}\text { BI: II, VIIb-VIII (Godwin 1975) NL: } 1000 \\
\text { B.C. (Pals 1977) }\end{array}$ \\
\hline $\begin{array}{l}\text { Cirsium cf. vulgare (Savi) } \\
\text { Ten. }\end{array}$ & RIA: $311+$ VA: $307 \mathrm{c}$ & \\
\hline
\end{tabular}


Table 2 continued

\begin{tabular}{|c|c|c|}
\hline Species & $\begin{array}{l}\text { Period, site number (cf. Table 1) and number } \\
\text { of macrofossils reported }\end{array}$ & $\begin{array}{l}\text { Reports on finds from neighbour countries. } \\
\text { Pollen zones and other periods are presented } \\
\text { as published by the authors cited }\end{array}$ \\
\hline Cirsium sp. & $\begin{array}{l}\text { GIA: } 242+\text { VA: } 304 \text { r EMA: } 172 \text { r } 428 \text { r } 429+ \\
\text { LMA: } 427+\end{array}$ & \\
\hline Cladium mariscus (L.) Pohl & $\begin{array}{l}\text { V: } 170 \mathrm{r} 407 \mathrm{r} 420 \mathrm{r} 539 \mathrm{cu} 549+555+603 \mathrm{ru} \\
826+905 \mathrm{r} 952+\text { VI, VII: } 170 \mathrm{r} 410 \mathrm{c} 420 \mathrm{r} 529 \mathrm{r} \\
549 \mathrm{c} 555+556 \mathrm{c} 561 \mathrm{r} 603 \mathrm{ru} 826+905 \mathrm{r} 944 \mathrm{r} \\
952 \mathrm{c} 170 \mathrm{r} 323+326 \mathrm{r} 407 \mathrm{r} 410 \mathrm{c} 420 \mathrm{r} 556 \mathrm{c} 603 \mathrm{ru} \\
906 \mathrm{r} 944 \mathrm{r} 952 \mathrm{r} \text { VIII: 323c 325+326c 410c 531c } \\
\text { 603ru IX: 324c 325+326r RIA: 301r LMA: } \\
217+\mathrm{u}\end{array}$ & \\
\hline $\begin{array}{l}\text { Clinopodium vulgare L. } \\
\text { (Satureja vulgaris (L.) } \\
\text { Fritsch) }\end{array}$ & VA: 304r 334r EMA: 521ru & $\begin{array}{l}\text { BI: II-III (Godwin 1975) BRD: 1st century } \\
\text { A.D. (Knörzer 1970) }\end{array}$ \\
\hline Cochlearia anglica $L$. & VA: $307 \mathrm{r}$ & \\
\hline Cochlearia officinalis $L$. & VA: $307+$ & BI: Late Weichselian (Godwin 1975) \\
\hline Conium maculatum L. & RIA: 1048 r VA: 110 r $307+$ & $\begin{array}{l}\text { BI: Roman Period (Godwin 1975), } 2130 \\
\pm 100 \text { B.P. (Greig 1979b) NL: } 3400-3300 \\
\text { B.C. (Zeist \& Palfenier-Vegter 1981) BRD: } \\
\text { 12-8 B.C. (Kučan 1981), 1st century A.D. } \\
\text { (Knörzer 1970) }\end{array}$ \\
\hline Coriandrum sativum L. & VA: 110r LMA: 406r & $\begin{array}{l}\text { BI: VIIb, Roman Period (Godwin 1975), } \\
\text { 2nd century A.D. (Wilson 1979), Roman } \\
\text { Period (Dickson et al. 1979), 2nd century } \\
\text { A.D. (Wilson 1979) BRD: 12-8 B.C. } \\
\text { (Kučan 1981), 1st century A.D. (Knörzer } \\
\text { 1967c, 1970), c. } 200 \text { A.D. (Körber-Grohne } \\
\text { 1979b) }\end{array}$ \\
\hline Cornus sanguinea $\mathrm{L}$. & VI, VII: 177 r 528+u 422ru VIII: 603ru 609r & BRD: 5500-4000 B.C. (Stalling 1983) \\
\hline $\begin{array}{l}\text { Cornus suecica L. } \\
\text { (Chamaepericlymenum } \\
\text { suecicum (L.) Ascherson \& } \\
\text { Graebner) }\end{array}$ & EMA: 521ru & $\begin{array}{l}\text { N: Late Viking (Tallantire 1979), Middle } \\
\text { Ages (Krzywinski \& Fægri 1979, Griffin } \\
\text { 1981) }\end{array}$ \\
\hline Corylus avellana $\mathrm{L}$. & $\begin{array}{l}\text { V: 539cu 550c VI, VII: 235ru 410r 501r 528+u } \\
549 \mathrm{c} 550 \mathrm{c} 944 \mathrm{r} \text { 159+ 235ru 410r 422cu 501+ } \\
508+\text { u 513+u 520ru VIII: 206r 404c 410r } \\
501+513+\text { u 531c 559ru 609+ 702c 703+987r } \\
\text { 1009r 1010r IX: 501r 513+ RIA: } 101+\text { GIA: } \\
242+244+\text { VA: } 172+202 \text { r 304c 307c 312c } \\
\text { 334c 985c EMA: } 172+428+429+431+ \\
521+\text { u LMA: } 218 r \text { r } 406+521 \mathrm{ru}\end{array}$ & $\begin{array}{l}\text { BI: IV-VIII (Godwin 1975) BRD: } 7200- \\
5500 \text { B.C. (Stalling 1983) P: Palaeolithic, } \\
\text { Neolithic, Bronze Age, Iron Age (Gluza \& } \\
\text { Wasylikowa 1977) }\end{array}$ \\
\hline Corylus sp. & V: 421ru VI, VII: 421ru VIII: 556r & \\
\hline $\begin{array}{l}\text { Crataegus laevigata (Poiret) } \\
\text { DC. (C. oxyacantha L.) }\end{array}$ & $V A: 304+312 c$ 334c EMA: $521+u$ & BRD: 12-8 B.C. (Kučan 1981) \\
\hline Crataegus monogyna Jacq. & VA: $312+334+$ & $\begin{array}{l}\text { BI: V-VIIa, VIII (Godwin 1975), } 3360 \pm 80 \\
\text { B.P. (Peglar \& Wilson 1978) BRD: } 12-8 \\
\text { B.C. (Kučan 1981), 1st century A.D. } \\
\text { (Knörzer 1970) P: Hallstatt (Gluza \& } \\
\text { Wasylikowa 1977) }\end{array}$ \\
\hline Crataegus sp. & VI, VII: 508ru VIII: 702r EMA: 427+ & $\begin{array}{l}\text { BI: VI, VIIb-VIII (Godwin 1975). } \\
\text { Crataegus monogyna Jacq. recorded from } \\
\text { NL: } 3400-3300 \text { B.C. (Zeist \& Palfenier- } \\
\text { Vegter 1981) }\end{array}$ \\
\hline Crepis capillaris (L.) Wallr. & PRIA: 115r & BI: VIIb (Godwin 1975) \\
\hline Crepis tectorum $\mathbf{L}$. & PRIA: $115+$ GIA: $712+$ & \\
\hline
\end{tabular}




\begin{tabular}{|c|c|c|}
\hline Species & $\begin{array}{l}\text { Period, site number (cf. Table 1) and number } \\
\text { of macrofossils reported }\end{array}$ & $\begin{array}{l}\text { Reports on finds from neighbour countries. } \\
\text { Pollen zones and other periods are presented } \\
\text { as published by the authors cited }\end{array}$ \\
\hline Crepis sp. & EMA: 172 r $430+$ LMA: $430+$ & \\
\hline Cuscuta epilinum Weihe & RIA: $303 c 311+316 c$ & \\
\hline Cuscuta sp. & LMA: $521 \mathrm{cu}$ & \\
\hline Cyperus fuscus L. & EMA: 521ru & $\begin{array}{l}\text { P: 11th-12th century A.D. (Wasylikowa } \\
1978 \text { ) }\end{array}$ \\
\hline $\begin{array}{l}\text { Cytisus scoparius (L.) Link } \\
\text { (Sarothamnus scoparius (L.) } \\
\text { Wimmer ex Koch) }\end{array}$ & VA: $307 \mathrm{r}$ & \\
\hline Dactylis glomerata $\mathrm{L}$. & GIA: $244+$ EMA: 992 r & BRD: c. 200 A.D. (Körber-Grohne 1979b) \\
\hline $\begin{array}{l}\text { Danthonia decumbens (L.) } \\
\text { DC. in Lam. \& DC. } \\
\text { (Sieglingia decumbens (L.) } \\
\text { Bernh.) }\end{array}$ & $\begin{array}{l}\text { VIII: } 230+\text { PRIA: } 115 \text { r } 301+\text { RIA: } 301+ \\
\text { GIA: } 135 \text { r 301+ 712r VA: 301c LMA: } 218+\end{array}$ & \\
\hline Daucus carota L. & $\begin{array}{l}\text { VIII: } 1065 \text { r RIA: } 311 \mathrm{c} \text { VA: } 304+307 \text { r } 334+ \\
\text { EMA: } 428 \text { r } 521+\text { u LMA: } 428+521+\mathrm{u}\end{array}$ & $\begin{array}{l}\text { BI: V, VIII (Godwin 1975) NL: } 1000 \text { B.C. } \\
\text { (Pals 1977) }\end{array}$ \\
\hline $\begin{array}{l}\text { Deschampsia cespitosa (L.) } \\
\text { Beauv. }\end{array}$ & PRIA: $115+$ VA: $110 \mathrm{r}$ & \\
\hline $\begin{array}{l}\text { Descurainia sophia (L.) } \\
\text { Webb ex Prantl in Engler \& } \\
\text { Prantl (Sisymbrium sophia } \\
\text { L.) }\end{array}$ & VA: $307 \mathrm{c} 334 \mathrm{c}$ & $\begin{array}{l}\text { BI: Roman Period (Godwin 1975) P: 10th- } \\
\text { 11th century A.D. (Wasylikowa 1978) }\end{array}$ \\
\hline Dianthus deltoides L. & EMA: $428 r$ & $\begin{array}{l}\text { BI: I (Godwin 1975) N: } 1000-1100 \text { A.D. } \\
\text { (Griffin 1975, 1977), 1150-1500 A.D. } \\
\text { (Griffin 1979a) }\end{array}$ \\
\hline Dryas octopetala $\mathbf{L}$. & $\begin{array}{l}\text { I: } 104 \mathrm{r} 113 \mathrm{r} 313+319 \mathrm{r} 416 \mathrm{ru} 417 \mathrm{ru} 510 \mathrm{c} 511 \mathrm{c} \\
\text { 513+548ru 714+ 720c II: 313r 705r 720r III: } \\
\text { 113r 152ru 319r 415ru 416ru 417ru 419ru 423cu } \\
424 \mathrm{cu} 426 \mathrm{ru} \mathrm{503ru} \mathrm{506+u} \mathrm{507cu} \mathrm{508+509r} \\
510+511+512+\mathrm{u} 513+523 \mathrm{r} 701 \mathrm{cu} 704 \mathrm{ru} \\
705 \mathrm{r} 706 \mathrm{ru} 714 \mathrm{r} \text { V: } 603 \mathrm{ru}\end{array}$ & BI: Late Weichselian, II-III (Godwin 1975) \\
\hline $\begin{array}{l}\text { Echinochloa crus-galli (L.) } \\
\text { Beauv. }\end{array}$ & $\begin{array}{l}\text { PRIA: } 114 r \text { 115+ RIA: 1064r EMA: } 245+ \\
\text { LMA: 406r }\end{array}$ & $\begin{array}{l}\text { First published as Setaria pumila (site } \\
\text { number 114). The identification corrected by } \\
\text { Helbæk (1954: 252) NL: } 805 \pm 65 \text { B.C. } \\
\text { (Zeist 1968) BRD: Band Ceramic (Knörzer } \\
\text { 1971a, 1974a, 1977), Hallstatt (Knörzer } \\
\text { 1971c, 1974b), 2nd-4th century A.D. } \\
\text { (Knörzer 1970) DDR: Late La Têne (Lange } \\
\text { 1975) }\end{array}$ \\
\hline Elatine hydropiper L. & LMA: 521ru & BI: 10th century A.D. (Wilson 1975) \\
\hline $\begin{array}{l}\text { Eleocharis palustris (L.) } \\
\text { Roemer \& Schultes (Scirpus } \\
\text { palustris L.) }\end{array}$ & $\begin{array}{l}\text { III: 161cu VI, VII: 513+u VIII: 323r IX: 327r } \\
\text { 328r 329c PRIA: 301r RIA: 139c 301c 306+ } \\
\text { 311+ 708r GIA: 301r VA: 301r 304c 307c 334c } \\
\text { EMA: 522+ LMA: 217ru 218r 406r 522+ }\end{array}$ & BI: I-IV, VI-VIII (Godwin 1975) \\
\hline $\begin{array}{l}\text { Eleocharis cf. palustris (L.) } \\
\text { Roemer \& Schultes }\end{array}$ & IX: 327c 328r RIA: 101+ & \\
\hline $\begin{array}{l}\text { Eleocharis palustris (L.) } \\
\text { Roemer \& Schultes et E. } \\
\text { uniglumis (Link) Schultes in } \\
\text { Schultes \& Schultes fil. }\end{array}$ & $\begin{array}{l}\text { GIA: } 242 \text { c } 243 \text { c } 244 \text { c VA: } 172 \text { r 202r EMA: } \\
172+245 \text { c } 427+428 c 429 c 430+431 \text { c LMA: } \\
241+245+427 c 428+429+430+\end{array}$ & \\
\hline $\begin{array}{l}\text { Eleocharis quinqueflora (F. } \\
\text { X. Hartmann) O. Schwarz } \\
\text { (Scirpus quinqueflorus F. X. } \\
\text { Hartmann) }\end{array}$ & LMA: $241+$ & $\begin{array}{l}\text { BI: Late Weichselian, VIIa (Godwin 1975) } \\
\text { N: Late Viking (Tallantire 1979) }\end{array}$ \\
\hline
\end{tabular}




\begin{tabular}{|c|c|c|}
\hline Species & $\begin{array}{l}\text { Period, site number (cf. Table 1) and number } \\
\text { of macrofossils reported }\end{array}$ & $\begin{array}{l}\text { Reports on finds from neighbour countries. } \\
\text { Pollen zones and other periods are presented } \\
\text { as published by the authors cited }\end{array}$ \\
\hline Eleocharis sp. & VIII: 127r EMA: 521cu LMA: 521cu & \\
\hline $\begin{array}{l}\text { Elymus caninus (L.) L. } \\
\text { (Agropyron caninum (L.) } \\
\text { Beauv., Roegneria canina } \\
\text { (L.) Nevski) }\end{array}$ & PRIA: $115+$ & BRD: Band Ceramic (Knörzer 1974a) \\
\hline cf. Elymus caninus (L.) L. & VIII: $1009 r$ & \\
\hline $\begin{array}{l}\text { Elymus cf. pycnanthus } \\
\text { (Godron) Melderis } \\
\text { (Agropyron littorale (Host) } \\
\text { Dum.) }\end{array}$ & VA: $307 \mathrm{c}$ & \\
\hline $\begin{array}{l}\text { Elymus repens (L.) Gould } \\
\text { (Agropyron repens (L.) } \\
\text { Beauv.) }\end{array}$ & RIA: 311c VA: $334 \mathrm{c}$ & $\begin{array}{l}\text { BRD: 12-8 B.C. (Kučan 1981) P: Hallstatt, } \\
\text { Roman Period and later (Gluza \& } \\
\text { Wasylikowa 1977) }\end{array}$ \\
\hline $\begin{array}{l}\text { Elymus cf. repens (L.) } \\
\text { Gould }\end{array}$ & GIA: $301 \mathrm{r}$ VA: $301 \mathrm{c} 304 \mathrm{c} 307 \mathrm{c}$ & \\
\hline Empetrum nigrum L. & $\begin{array}{l}\text { I: } 155+\text { II: } 104 \mathrm{r} 113+155+827+\text { III: } 113+ \\
\text { 138ru 155r 158+u 161+u 512ru 540+ 995r IV: } \\
\text { 235cu 421ru 995r VI, VII: 501cu 506ru 501+ } \\
\text { VIII: 501+ IX: 501c 508+ PRIA: 301r RIA: } \\
\text { 128r 301+ GIA: 301r VA: 301r EMA: } 172+ \\
245+\end{array}$ & BI: I-VIII (Godwin 1975) \\
\hline Epilobium cf. hirsutum $\mathbf{L}$. & VA: $304 \mathrm{r}$ & $\begin{array}{l}\text { Epilobium hirsutum recorded from NL: } \\
\text { before } 1400 \text { B.C. (Pals et al. 1980) }\end{array}$ \\
\hline Epilobium montanum L. & RIA: $306 \mathrm{r}$ & \\
\hline Epilobium palustre L. & VA: $304 \mathrm{r} 307+334 \mathrm{c}$ & $\begin{array}{l}\text { BI: II (Godwin 1975) NL: } 1100 \text { B.C. (Pals et } \\
\text { al. 1980) BRD: 1st century B.C. (Knörzer } \\
\text { 1970) }\end{array}$ \\
\hline Epilobium sp. & VA: 202 r $334+$ EMA: $430 c 431+$ LMA: $430 c$ & \\
\hline Erica tetralix L. & $\begin{array}{l}\text { RIA: 301c GIA: 301r VA: } 301+\text { EMA: } 172+ \\
\text { 521cu LMA: } 522 \text { r }\end{array}$ & $\begin{array}{l}\text { BI: I-VIII (Godwin 1975) BRD: 1st-2nd } \\
\text { century A.D. (Körber-Grohne 1967) }\end{array}$ \\
\hline $\begin{array}{l}\text { Eriophorum angustifolium } \\
\text { Honckeny }\end{array}$ & IX: $176 \mathrm{c} 332 \mathrm{r} 501+$ & BI: II, IV, VIIa-VIII (Godwin 1975) \\
\hline $\begin{array}{l}\text { Eriophorum cf. angustifolium } \\
\text { Honckeny }\end{array}$ & IX: $328 \mathrm{r}$ & \\
\hline Eriophorum vaginatum $\mathrm{L}$. & $\begin{array}{l}\text { III: } 161+\text { u IV: } 175 \mathrm{c} \text { V: } 175 \mathrm{c} \text { VI, VII: } 175 \mathrm{c} \\
501+175 \mathrm{c} 501+502+944 \mathrm{r} \text { VIII: } 162 \mathrm{r} 164 \mathrm{r} \\
176 \mathrm{c} 180+501+502+508 \mathrm{cu} 559 \mathrm{r} 944 \mathrm{r} \text { IX: } \\
162 \mathrm{r} 164 \mathrm{r} 167 \mathrm{r} 176 \mathrm{c} 180+332 \mathrm{c} 501+502+ \\
\text { 508c 513c 559r 944r RIA: 128r VA: } 334 \mathrm{c}\end{array}$ & BI: II, IV, VI-VIII (Godwin 1975) \\
\hline Eriophorum sp. & EMA: 521ru & \\
\hline $\begin{array}{l}\text { Erodium cicutarium (L.) } \\
\text { L'Her. in Aiton }\end{array}$ & VIII: 214 r VA: $304 r$ 334r & \\
\hline Erysimum cheiranthoides L. & PRIA: 111r 114r 115r GIA: $712+$ & NL: 500 B.C. -200 A.D. (Zeist 1974) \\
\hline Eupatorium cannabinum L. & VI, VII: $528 \mathrm{cu} 549 \mathrm{c} 508 \mathrm{ru}$ VA: $334 \mathrm{c}$ & $\begin{array}{l}\text { BI: V-VIII (Godwin 1975) BRD: } 7200-5500 \\
\text { B.C. (Stalling 1983) }\end{array}$ \\
\hline Euphorbia helioscopia L. & $\begin{array}{l}\text { RIA: } 301 \text { r 311r GIA: 301r VA: 202r 301r 304r } \\
\text { 307c 320+ 334+ 985r EMA: } 427+428+429+ \\
\text { 521+u LMA: } 218 \text { r } 241+406 r \text { r } 428+429+ \\
\text { 521ru }\end{array}$ & $\begin{array}{l}\text { NL: } 200 \text { B.C.-250 A.D. (Zeist 1974) BRD: } \\
\text { c. } 200 \text { B.C. (Knörzer 1979a), 12-8 B.C. } \\
\text { (Kučan 1981), 1st century A.D. (Knörzer } \\
\text { 1970),1st-2nd century A.D. (Körber-Grohne } \\
\text { 1967) }\end{array}$ \\
\hline cf. Euphorbia helioscopia L. & VIII: $1009 \mathrm{r}$ & \\
\hline
\end{tabular}


Table 2 continued

\begin{tabular}{|c|c|c|}
\hline Species & $\begin{array}{l}\text { Period, site number (cf. Table 1) and number } \\
\text { of macrofossils reported }\end{array}$ & $\begin{array}{l}\text { Reports on finds from neighbour countries. } \\
\text { Pollen zones and other periods are presented } \\
\text { as published by the authors cited }\end{array}$ \\
\hline Euphorbia lathyris L. & EMA: $427+$ & \\
\hline Euphorbia sp. & EMA: $245 c$ LMA: $245+$ & \\
\hline Fagus sylvatica $\mathrm{L}$. & $\begin{array}{l}\text { VI, VII: } 501 \mathrm{cu} 501 \mathrm{cu} \text { VIII: } 501 \mathrm{c} \text { IX: } 501 \mathrm{c} \\
502+513 \mathrm{c} \text { GIA: } 553 \mathrm{c} \text { VA: } 304+312 \mathrm{c} 334 \mathrm{c} \\
\text { EMA: } 564 \mathrm{c}\end{array}$ & $\begin{array}{l}\text { P: Palaeolithic, Mesolithic (Gluza \& } \\
\text { Wasylikowa 1977) }\end{array}$ \\
\hline $\begin{array}{l}\text { Festuca arundinacea } \\
\text { Schreber }\end{array}$ & VA: $307 r$ & \\
\hline Festuca cf. pratensis Hudson & VA: 985r EMA: $992+$ & $\begin{array}{l}\text { BRD: c. } 200 \text { A.D. (Körber-Grohne } 1979 \text { b) } \\
\text { Festuca pratensis recorded from P: Early } \\
\text { Medieval Period (Gluza \& Wasylikowa } \\
\text { 1977) }\end{array}$ \\
\hline Festuca rubra $\mathrm{L}$. & RIA: $301 \mathrm{c} 311 \mathrm{c}$ VA: $304 \mathrm{c} 307 \mathrm{c} 320+334 \mathrm{c}$ & $\begin{array}{l}\text { NL: } 600-400 \text { B.C. (Zeist 1974) BRD: c. } 200 \\
\text { B.C. (Knörzer 1979a), 1st-2nd century A.D. } \\
\text { (Körber-Grohne 1967, Knörzer 1979b) }\end{array}$ \\
\hline Festuca cf. rubra L. & EMA: $172 \mathrm{r}$ & \\
\hline Festuca sp. & RIA: $309 r$ & \\
\hline Ficus carica L. & EMA: 521ru LMA: $521+u$ & $\begin{array}{l}\text { BI: Roman, Medieval Period (Godwin } \\
\text { 1975), Roman Period (Willcox 1977, } \\
\text { Dickson et al. 1979), 1st-2nd century A.D. } \\
\text { (Kenward \& Williams 1979), 2nd century } \\
\text { A.D. (Wilson 1979) BRD: 12-8 B.C. } \\
\text { (Kučan 1981), 1st century A.D. (Knörzer } \\
\text { 1967b, 1967c, 1970), 1st century and later } \\
\text { (Knörzer 1973), c. 200 A.D. (Körber- } \\
\text { Grohne 1979b) P: 13th century (Wieserowa } \\
\text { 1979) N: 1200-1250 A.D. (Griffin 1979b) }\end{array}$ \\
\hline $\begin{array}{l}\text { Filipendula ulmaria (L.) } \\
\text { Maxim. (Spiraea ulmaria L.) }\end{array}$ & $\begin{array}{l}\text { VIII: 421ru 603ru VA: } 304 \mathrm{c} 334 \mathrm{c} 985 \text { r LMA: } \\
\text { 241c }\end{array}$ & $\begin{array}{l}\text { BI: I-IV, VI-VIII (Godwin 1975) BRD: } \\
5500-4000 \text { B.C. and later (Stalling 1983) }\end{array}$ \\
\hline Fragaria vesca $L$. & $\begin{array}{l}\text { VIII: } 531 c \text { GIA: } 243+244 c \text { VA: } 304+312 c \\
\text { 334c EMA: } 428 c 429 c 431+\text { LMA: } 241+406 c \\
427 \text { c } 428 c 430+\end{array}$ & BI: VIIa-VIII (Godwin 1975) \\
\hline Fragaria sp. & EMA: 521cu LMA: 521cu & \\
\hline $\begin{array}{l}\text { Frangula alnus Miller } \\
\text { (Rhamnus frangula L.) }\end{array}$ & $\begin{array}{l}\text { V: 539ru VI, VII: 422ru 944r VIII: 328r 603ru } \\
\text { IX: 513c LMA: 217ru }\end{array}$ & BI: V-VIII (Godwin 1975) \\
\hline Frangula cf. alnus Miller & VI, VII: 549r & \\
\hline Fraxinus excelsior $\mathbf{L}$. & $\begin{array}{l}\text { VI, VII: 410r 159r 410r 422ru 508ru VIII: 410r } \\
\text { IX: } 513+\text { VA: } 306 r\end{array}$ & $\begin{array}{l}\text { P: Palaeolithic, Mesolithic (Gluza \& } \\
\text { Wasylikowa 1977) }\end{array}$ \\
\hline Fumaria officinalis $\mathbf{L}$. & $\begin{array}{l}\text { PRIA: } 111+115 \text { r RIA: } 107 \text { r } 708+\text { VA: } 103 \text { r } \\
\text { LMA: } 521 \text { ru } 1025+\end{array}$ & $\begin{array}{l}\text { BI: VIIb-VIII (Godwin 1975), Roman } \\
\text { Period (Greig 1980) BRD: 12-8 B.C. } \\
\text { (Kučan 1981) }\end{array}$ \\
\hline $\begin{array}{l}\text { Galeopsis bifida Boenn., G. } \\
\text { speciosa Miller et G. tetrahit } \\
\text { L. }\end{array}$ & RIA: $115+708+$ GIA: $712+$ VA: $110 \mathrm{r}$ & \\
\hline Galeopsis ladanum L. & RIA: 139r EMA: 992r & BRD: c. 200 B.C. (Knörzer 1979a) \\
\hline Galeopsis segetum Necker & VA: $334+$ & BRD: c. 900 A.D. (Kučan 1979) \\
\hline Galeopsis cf. speciosa Miller & LMA: $218 c 522 \mathrm{r}$ & \\
\hline $\begin{array}{l}\text { Galeopsis speciosa Miller et } \\
\text { G. tetrahit L. }\end{array}$ & $\begin{array}{l}\text { RIA: } 311 c \text { GIA: } 135 r \text { VA: } 304 c 307+334 c \\
\text { LMA: } 406+\end{array}$ & $\begin{array}{l}\text { BI: Early Iron Age, Roman Period (Helbak } \\
\text { 1952) NL: } 500 \text { B.C.-200 A.D. (Zeist 1974) } \\
\text { BRD: 12-8 B.C. (Kučan 1981), c. } 200 \text { A.D. } \\
\text { (Körber-Grohne 1979b) }\end{array}$ \\
\hline
\end{tabular}

(continued) 


\begin{tabular}{|c|c|c|}
\hline Species & $\begin{array}{l}\text { Period, site number (cf. Table 1) and number } \\
\text { of macrofossils reported }\end{array}$ & $\begin{array}{l}\text { Reports on finds from neighbour countries. } \\
\text { Pollen zones and other periods are presented } \\
\text { as published by the authors cited }\end{array}$ \\
\hline Galeopsis tetrahit L. & VIII: 425c GIA: 1087ru VA: 985r EMA: $992+$ & $\begin{array}{l}\text { BI: II-IV, VIIa-VIII (Godwin 1975) P: } \\
\text { Hallstatt and later (Gluza \& Wasylikowa } \\
\text { 1977) }\end{array}$ \\
\hline Galeopsis cf. tetrahit $\mathbf{L}$. & VIII: 543r RIA: $107+108 \mathrm{c}$ LMA: $218 \mathrm{c}$ & \\
\hline Galeopsis sp. & $\begin{array}{l}\text { VIII: } 317 \text { r PRIA: } 109+114+\text { RIA: } 301 \mathrm{c} 308 \mathrm{r} \\
\text { 309+ GIA: } 242+301 \mathrm{r} \text { VA: } 103+172+301+ \\
\text { EMA: } 172+245 \mathrm{c} 427+428+429+430+ \\
431+521 \mathrm{cu} \text { LMA: } 241 \mathrm{c} 245+427+521 \mathrm{ru}\end{array}$ & \\
\hline Galium aparine $\mathbf{L}$. & $\begin{array}{l}\text { VIII: 411r 609+ IX: 1087r PRIA: 301r RIA: } \\
\text { 301r 708r GIA: 301r VA: 301r 312r 334c } \\
\text { LMA: 406r 809r }\end{array}$ & $\begin{array}{l}\text { BI: VIIb-VIII (Godwin 1975) NL: 3400- } \\
3300 \text { B.C. (Zeist \& Palfenier-Vegter 1981) } \\
\text { BRD: Band Ceramic (Knörzer 1977) P: } \\
\text { Neolithic (Gluza \& Wasylikowa 1977) }\end{array}$ \\
\hline cf. Galium aparine L. & VIII: $317 \mathrm{r}$ & \\
\hline Galium mollugo L. & RIA: $303+$ VA: $304+334+$ & $\begin{array}{l}\text { BI: II (Godwin 1975) BRD: 1st century } \\
\text { A.D. (Knörzer 1970), 2nd century A.D. } \\
\text { (Knörzer 1979b) }\end{array}$ \\
\hline Galium cf. mollugo L. & PRIA: 301r VA: 301r & \\
\hline Galium palustre $\mathbf{L}$. & RIA: $311 \mathrm{c}$ VA: $103 \mathrm{r} 304 \mathrm{c} 307 \mathrm{c} 334 \mathrm{c}$ & $\begin{array}{l}\text { BI: IV/V, Roman Period (Godwin 1975), } \\
\text { Iron Age (Greig 1979a, 1979b) NL: 3400- } \\
\text { 3300 B.C. (Zeist \& Palfenier-Vegter 1981) } \\
\text { BRD: Band Ceramic (Knörzer 1977), 1st- } \\
\text { 2nd century A.D. (Knörzer 1970, 1979b, } \\
\text { Körber-Grohne 1967) }\end{array}$ \\
\hline Galium cf. palustre L. & $\begin{array}{l}\text { PRIA: 301r RIA: 301c GIA: 301r VA: 202r } \\
\text { 301+ }\end{array}$ & \\
\hline $\begin{array}{l}\text { Galium spurium L. (G. } \\
\text { vaillantii } D C \text {.) }\end{array}$ & RIA: $708+$ GIA: 712c VA: $985+$ LMA: $809+$ & $\begin{array}{l}\text { BI: Late Bronze Age (Godwin 1975) BRD: } \\
\text { Band Ceramic (Knörzer 1974a, 1977), } \\
\text { Neolithic (Knörzer 1971a), Hallstatt } \\
\text { (Knörzer 1974b), c. 200 B.C. (Knörzer } \\
\text { 1979a), c.200 A.D. (Körber-Grohne 1979b) } \\
\text { P: Hallstatt and later (Gluza \& Wasylikowa } \\
\text { 1977) }\end{array}$ \\
\hline Galium sp. & $\begin{array}{l}\text { VIII: 976r VA: 202r EMA: 428r LMA: } 241+ \\
\text { 521ru }\end{array}$ & \\
\hline $\begin{array}{l}\text { Genista anglica } \mathrm{L} \text {. et } \mathrm{G} \text {. } \\
\text { pilosa } \mathrm{L} \text {. }\end{array}$ & VIII: $229 \mathrm{r}$ & \\
\hline Geranium columbinum $\mathbf{L}$. & VA: $334+$ & \\
\hline Geranium molle $\mathrm{L}$. & VA: $985 r$ & \\
\hline Geranium robertianum $\mathbf{L}$. & VA: $307 r$ & \\
\hline Geum sp. & V: 421ru VI, VII: 421 ru & $\begin{array}{l}\text { Geum rivale or G. urbanum recorded from } \\
\text { BI: I-II, IV-VI (Godwin 1975) }\end{array}$ \\
\hline Glaux maritima L. & $\begin{array}{l}\text { RIA: 301r 311c GIA: 301r VA: 304r 307c } \\
320+334 \text { r }\end{array}$ & $\begin{array}{l}\text { BI: I-III, VIIb (Godwin 1975) NL: } 600-400 \\
\text { B.C. (Zeist 1974) BRD: 1st-2nd century } \\
\text { A.D. (Körber-Grohne 1967) }\end{array}$ \\
\hline Glechoma hederaceae L. & LMA: $504+$ & $\begin{array}{l}\text { BRD: 1155-1227 A.D. (Lynch \& Paap 1982) } \\
\text { P: 13th century A.D. (Wieserowa 1979) }\end{array}$ \\
\hline Glyceria fluitans (L.) R. Br. & VA: $334 \mathrm{r}$ & $\begin{array}{l}\text { NL: } 3400-3300 \text { B.C. (Zeist \& Palfenier- } \\
\text { Vegter 1981) BRD: Neolithic (Bertsch } \\
\text { 1954), 3000-900 B.C. (Stalling 1983), c. } 200 \\
\text { A.D. (Körber-Grohne 1979b) }\end{array}$ \\
\hline
\end{tabular}


Table 2 continued

\begin{tabular}{|c|c|c|}
\hline Species & $\begin{array}{l}\text { Period, site number (cf. Table 1) and number } \\
\text { of macrofossils reported }\end{array}$ & $\begin{array}{l}\text { Reports on finds from neighbour countries. } \\
\text { Pollen zones and other periods are presented } \\
\text { as published by the authors cited }\end{array}$ \\
\hline $\begin{array}{l}\text { Glyceria maxima (Hartmann) } \\
\text { Holmberg }\end{array}$ & VA: $334 \mathrm{c}$ & $\begin{array}{l}\text { BI: VIII (Godwin 1975) NL: 100-250 A.D. } \\
\text { (Zeist 1974) BRD: 12-8 B.C. (Kučan 1981), } \\
\text { 1st century A.D. (Knörzer 1970) }\end{array}$ \\
\hline Hieracium pilosella L. & VA: $304 \mathrm{r} 334 \mathrm{r}$ & \\
\hline Hieracium umbellatum L. & RIA: $708 \mathrm{r}$ & \\
\hline Hieracium sp. & VA: $304+334+$ EMA: $521+$ u LMA: $430+$ & \\
\hline Hippophae rhamnoides L. & IV: $827+$ & BI: I-II (Godwin 1975) \\
\hline cf. Hippophae rhamnoides $L$. & VIII: $1089 \mathrm{r}$ & \\
\hline Hippuris vulgaris $\mathbf{L}$. & $\begin{array}{l}\text { II: 155r 416ru 417ru 419+510r 511r 523r III: } \\
\text { 104r 152ru 153ru 158+u 417ru 506ru IV: 209r } \\
\text { VI, VII: 549r IX: 501+ EMA: 521ru LMA: } \\
217+\text { u } 521+u\end{array}$ & BI: I-VIII (Godwin 1975) \\
\hline Hippuris sp. & IV: 421ru & \\
\hline Holcus lanatus $\mathbf{L}$. & PRIA: 115+ LMA: 521ru & $\begin{array}{l}\text { BI: IV/V, VIII (Godwin 1975) NL: } 500 \\
\text { B.C.-200 A.D. (Zeist 1974) }\end{array}$ \\
\hline Holcus sp. & VIII: $127+$ & \\
\hline $\begin{array}{l}\text { Hordeum cf. distichon L. } \\
\text { 'Two-rowed? hulled barley' }\end{array}$ & EMA: $428+$ & $\begin{array}{l}\text { Hordeum distichon recorded from NL: 8th- } \\
\text { 9th century A.D. (Zeist 1968) BRD: 10th- } \\
\text { early 11th century (Wittmack \& Buchwald } \\
\text { 1902) DDR: 3rd-4th century A.D. } \\
\text { (Schiemann 1957) }\end{array}$ \\
\hline $\begin{array}{l}\text { Hordeum secalinum Schreber } \\
\text { (H. nodosum L.) }\end{array}$ & VA: $307+$ & \\
\hline $\begin{array}{l}\text { Hordeum vulgare L. 'Six- } \\
\text { rowed hulled barley' (H. } \\
\text { tetrastichum Kcke.) }\end{array}$ & 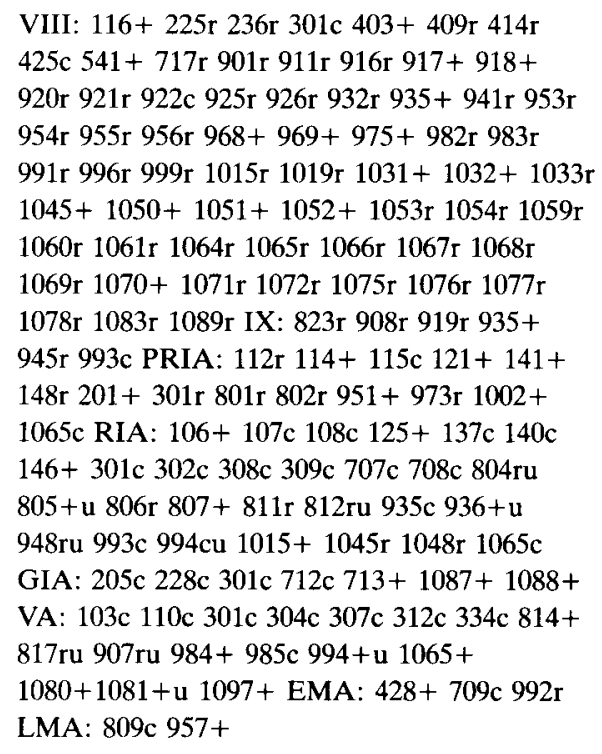 & $\begin{array}{l}\text { BI: VIIb-VIII (Godwin 1975) BRD: Bronze } \\
\text { Age (Behre 1982) }\end{array}$ \\
\hline $\begin{array}{l}\text { Hordeum vulgare L. var. } \\
\text { nudum 'Six-rowed naked } \\
\text { barley' (H. tetrastichum } \\
\text { Kcke. f. nudum, } \mathrm{H} \text {. } \\
\text { polystichum var. coeleste) }\end{array}$ & $\begin{array}{l}\text { VIII: } 116+143 \mathrm{r} 145 \mathrm{r} 204+208 \mathrm{c} 225 \mathrm{r} 226 \mathrm{r} \\
227 \mathrm{r} 231 \mathrm{r} 232 \mathrm{r} 236 \mathrm{r} 301+317 \mathrm{r} 401+403 \mathrm{c} 405 \mathrm{c} \\
409+413 \mathrm{r} 425 \mathrm{c} 505 \mathrm{r} 514 \mathrm{r} 515 \mathrm{r} 526 \mathrm{r} 532 \mathrm{r} 533 \mathrm{r} \\
536 \mathrm{r} 541+542 \mathrm{r} 544 \mathrm{r} 601 \mathrm{r} 605 \mathrm{r} 608 \mathrm{r} 609+702 \mathrm{c} \\
703 \mathrm{c} 715 \mathrm{r} 716 \mathrm{r} 717 \mathrm{r} 808 \mathrm{r} 810 \mathrm{r} 824+825+903 \mathrm{r} \\
904 \mathrm{r} 909 \mathrm{r} 910 \mathrm{r} 912 \mathrm{r} 917 \mathrm{r} 918 \mathrm{r} 922 \mathrm{c} 925+926 \mathrm{r} \\
929 \mathrm{r} 930 \mathrm{r} 933 \mathrm{r} 937 \mathrm{r} 938 \mathrm{r} 939 \mathrm{r} 940 \mathrm{r} 942 \mathrm{r} 946+ \\
947+948 \mathrm{r} 953 \mathrm{r} 954 \mathrm{r} 956 \mathrm{r} 958+959+961 \mathrm{r} \\
965+966+968+969 \mathrm{r} 974 \mathrm{r} 975+976+978+ \\
979 \mathrm{r} 982 \mathrm{r} 986 \mathrm{r} 1000+1001+1003 \mathrm{r} 1004+ \\
1005 \mathrm{r} 1006 \mathrm{r} 1009+1010+1020+1023 \mathrm{r} 1024 \mathrm{r}\end{array}$ & $\begin{array}{l}\text { BI: VIIb-VIII (Godwin 1975) NL: } 3400- \\
\text { 3300 B.C. (Zeist \& Palfenier-Vegter 1981) } \\
\text { BRD: Bronze Age (Behre 1982) }\end{array}$ \\
\hline
\end{tabular}


Table 2 continued

\begin{tabular}{|c|c|c|}
\hline Species & $\begin{array}{l}\text { Period, site number (cf. Table 1) and number } \\
\text { of macrofossils reported }\end{array}$ & $\begin{array}{l}\text { Reports on finds from neighbour countries. } \\
\text { Pollen zones and other periods are presented } \\
\text { as published by the authors cited }\end{array}$ \\
\hline $\begin{array}{l}\text { Hordeum vulgare L. var. } \\
\text { nudum 'Six-rowed naked } \\
\text { barley' } \\
\text { (continued) }\end{array}$ & $\begin{array}{l}\text { 1026r } 1027+1031 \mathrm{c} 1032 \mathrm{c} 1034+1035 \mathrm{r} 1038 \mathrm{r} \\
1039 \mathrm{r} 1040 \mathrm{r} 1041 \mathrm{r} 1042 \mathrm{r} 1043 \mathrm{r} 1044 \mathrm{r} 1045 \mathrm{r} \\
1050+1051+1062 \mathrm{r} 1063 \mathrm{r} 1065+1069+1072 \mathrm{r} \\
1075 \mathrm{r} 1076 \mathrm{r} 1078 \mathrm{r} 1079 \mathrm{r} 1083 \mathrm{r} 1084 \mathrm{r} 1085 \mathrm{r} \\
1086+1089+1093 \mathrm{r} 1094 \mathrm{r} \text { IX: } 813 \mathrm{r} 993 \mathrm{c} \\
\text { PRIA: } 102 \mathrm{c} 111 \mathrm{c} 112 \mathrm{c} 114+115 \mathrm{c} 121+141 \mathrm{c} \\
148+201 \mathrm{c} 301 \mathrm{c} 802 \mathrm{r} 1002 \mathrm{r} 1065+\text { RIA: } 107 \mathrm{c} \\
108 \mathrm{c} 125 \mathrm{c} 137 \mathrm{cu} 140+146+301 \mathrm{c} 302 \mathrm{c} 308 \mathrm{c} \\
309 \mathrm{c} 707 \mathrm{c} 708+804 \mathrm{ru} 807+810 \mathrm{ru} 935+ \\
936+\mathrm{u} 980 \mathrm{r} 993 \mathrm{c} 994 \mathrm{cu} 1065+1087+1088+ \\
\text { GIA: } 1087+1088 \mathrm{r} \text { VA: } 304 \mathrm{c} 334 \mathrm{r} 814 \mathrm{r} 994 \mathrm{cu} \\
1064+1065+1080+1081+\mathrm{u} 1097+\text { LMA: } \\
\text { 957r }\end{array}$ & \\
\hline $\begin{array}{l}\text { Hordeum vulgare L. 'Hulled } \\
\text { or naked barley' }\end{array}$ & $\begin{array}{l}\text { VIII: } 116+203 \mathrm{r} 317 \mathrm{r} 404+915 \mathrm{r} 918 \mathrm{r} 922 \mathrm{c} \\
\text { 1031r } 1089+\text { IX: } 935+\text { PRIA: } 102 \mathrm{c} 301+951 \mathrm{r} \\
\text { 1065+ RIA: } 106 \mathrm{r} 108 \mathrm{c} 128+140 \mathrm{c} 301 \mathrm{c} 303 \mathrm{c} \\
\text { 308c 311c 315c 602c } 936+\mathrm{u} 1065+\mathrm{u} \text { GIA: } 242 \mathrm{r} \\
\text { 1087r 1088r VA: 320c } 994 \mathrm{cu} 1064 \mathrm{r} \text { 1065r } \\
\text { EMA: } 172 \mathrm{r} 245+428 \mathrm{c} 429+710 \mathrm{c} \text { LMA: } 245+ \\
428+429+\end{array}$ & $\begin{array}{l}\text { P: Neolithic, Bronze Age (Gluza \& } \\
\text { Wasylikowa 1977) S: Neolithic (Frödin 1910) }\end{array}$ \\
\hline Hordeum sp. & $\begin{array}{l}\text { VIII: } 206+213 \text { ru } 221+301+425 \mathrm{c} 917 \mathrm{r} 953 \mathrm{r} \\
\text { 954r 956r 958r 959r 1032r PRIA: 301r RIA: } \\
\text { 301+306r 316+602c 935+ GIA: 301c 306r } \\
\text { VA: } 202 \mathrm{c} 301+306 \mathrm{r} \text { EMA: } 992 \mathrm{c}\end{array}$ & \\
\hline Humulus lupulus L. & $\begin{array}{l}\text { VI, VII: 528ru GIA: } 244+\text { VA: } 304 \mathrm{c} 307+ \\
312 \text { c } 334 \mathrm{c} \text { EMA: } 428 \mathrm{c} 429+431+521 \mathrm{cu} \\
\text { LMA: } 218+241+406+430+521 \mathrm{cu} 522+\end{array}$ & BI: VIIb-VIII (Godwin 1975) \\
\hline Hydrocharis morsus-ranae L. & RIA: $301+$ & \\
\hline Hydrocotyle vulgaris L. & $\begin{array}{l}\text { III: 161ru VI, VII: 326+ VIII: 326+ IX: 209r } \\
\text { 326r 331r 332+ 501r 711r RIA: } 101+301 \mathrm{r} \\
\text { 311c VA: 301r 307r 334+ EMA: 564r }\end{array}$ & \\
\hline Hyoscyamus niger $L$. & $\begin{array}{l}\text { PRIA: 301c RIA: 301c 311r GIA: } 244+\text { VA: } \\
\text { 172r 307r EMA: } 245+428+429 \mathrm{c} 430+521+\mathrm{u} \\
\text { LMA: } 147+428+430+517+\mathrm{u} 521 \mathrm{cu} 522 \mathrm{r} \\
\text { 1025r }\end{array}$ & $\begin{array}{l}\text { BI: } 2130 \pm 100 \text { B.P. (Greig 1979b) BRD: c. } \\
\text { 2500 B.C. (Piening 1979), 12-8 B.C. (Kučan } \\
\text { 1981) P: Hallstatt and later (Gluza \& } \\
\text { Wasylikowa 1977) }\end{array}$ \\
\hline Hyoscyamus sp. & VA: $110 \mathrm{c} 154 \mathrm{c}$ & \\
\hline $\begin{array}{l}\text { Hypericum maculatum } \\
\text { Crantz }\end{array}$ & VA: $334 c$ & BI: VII/VIII (Godwin 1975) \\
\hline $\begin{array}{l}\text { Hypericum cf. maculatum } \\
\text { Crantz }\end{array}$ & VA: $304+$ & \\
\hline Hypericum perforatum $\mathrm{L}$. & VA: $334 c$ & $\begin{array}{l}\text { BI: Roman Period (Godwin 1975) BRD: } \\
\text { 12-8 B.C. (Kučan 1981), 1st century A.D. } \\
\text { (Knörzer 1970) P: Hallstatt and later (Gluza } \\
\text { \& Wasylikowa 1977) S: Middle Iron Age } \\
\text { (Helbæk 1955b) }\end{array}$ \\
\hline Hypericum tetrapterum Fries & VA: $304 \mathrm{r} 334 \mathrm{c}$ & $\begin{array}{l}\text { BI: VIIa-VIII (Godwin 1975) NL: } 1000 \\
\text { B.C. (Pals 1977) BRD: 12-8 B.C. (Kučan } \\
\text { 1981), 1st-2nd century A.D. (Körber- } \\
\text { Grohne 1967, 1979b) }\end{array}$ \\
\hline Hypericum sp. & $\begin{array}{l}\text { VIII: 229r GIA: 242r VA: } 172 \text { r 304+ EMA: } \\
\text { 172r } 427+428+429+\text { LMA: } 241 \mathrm{c} 427+428 \mathrm{c}\end{array}$ & $\begin{array}{l}\text { BI: III-IV, VIIa-VIII (Godwin 1975) NL: c. } \\
\text { 2700 B.P. (Geel et al. 1983) P: Neolithic } \\
\text { (Gluza \& Wasylikowa 1977) }\end{array}$ \\
\hline Hypochoeris cf. glabra L. & VA: $334 r$ & $\begin{array}{l}\text { Hypochoeris glabra recorded from BI: 2nd } \\
\text { century A.D. (Wilson 1979) }\end{array}$ \\
\hline
\end{tabular}




\begin{tabular}{|c|c|c|}
\hline Species & $\begin{array}{l}\text { Period, site number (cf. Table 1) and number } \\
\text { of macrofossils reported }\end{array}$ & $\begin{array}{l}\text { Reports on finds from neighbour countries. } \\
\text { Pollen zones and other periods are presented } \\
\text { as published by the authors cited }\end{array}$ \\
\hline Iris pseudacorus L. & $\begin{array}{l}\text { VI, VII: } 549 \text { r } 422 \text { ru IX: } 513+\text { GIA: } 244+\text { VA: } \\
304 c \text { c } 307+334 \text { c } 985 \text { r }\end{array}$ & \\
\hline Isatis tinctoria $\mathbf{L}$. & RIA: $107 \mathrm{c} 108+125+803 \mathrm{ru} 806 \mathrm{r}$ & $\begin{array}{l}\text { BRD: 6th-5th century B.C. (Körber-Grohne } \\
\text { 1981) }\end{array}$ \\
\hline Juglans regia $\mathrm{L}$. & VA: $304+312 \mathrm{c} 334 \mathrm{c} 985 \mathrm{r}$ & $\begin{array}{l}\text { BI: Roman Period (Godwin 1975), 1st-3rd } \\
\text { century A.D. (Willcox 1977) BRD: 1st } \\
\text { century A.D. (Knörzer 1970), c. } 200 \text { A.D. } \\
\text { (Körber-Grohne 1979b), 6th century A.D. } \\
\text { (Hopf 1965), 6th-7th century A.D. (Bertsch } \\
\text { 1927), 10th-11th century A.D. (Wittmack \& } \\
\text { Buchwald 1902) P: Roman Period and later } \\
\text { (Gluza \& Wasylikowa 1977) N: Viking Age } \\
\text { (Holmboe 1927) }\end{array}$ \\
\hline Juncus anceps Laharpe & VA: $334 c$ & \\
\hline Juncus articulatus L. & RIA: $311 \mathrm{c}$ VA: $304 \mathrm{c} 334 \mathrm{c}$ & $\begin{array}{l}\text { BI: Late 2nd century A.D. (Wilson 1979) } \\
\text { NL: } 600-400 \text { B.C. (Zeist 1974) BRD: 12-8 } \\
\text { B.C. (Kučan 1981) }\end{array}$ \\
\hline Juncus cf. articulatus $\mathbf{L}$. & RIA: 301c GIA: 301r & \\
\hline Juncus bufonius $\mathrm{L}$. & $\begin{array}{l}\text { PRIA: 301r RIA: 301c 311c GIA: } 244+301 \mathrm{c} \\
\text { VA: } 301 \mathrm{c} 304 \mathrm{c} 307 \mathrm{c} 334 \mathrm{c} \text { EMA: } 245 \mathrm{c} 428 \mathrm{c} \\
\text { 429c } 431+\text { LMA: } 427 \mathrm{c} 428+429+\end{array}$ & $\begin{array}{l}\text { BI: I, III-IV, VIIb (Godwin 1975) NL: } 1400 \\
\text { B.C. (Pals et al. 1980), } 2800 \pm 50 \text { B.P. (Geel } \\
\text { et al. 1983) BRD: 12-8 B.C. (Kučan 1981) }\end{array}$ \\
\hline Juncus cf. compressus Jacq. & RIA: $311 \mathrm{c}$ VA: $304+334 \mathrm{c}$ & $\begin{array}{l}\text { Juncus compressus recorded from BRD: c. } \\
200 \text { A.D. (Körber-Grohne 1979b) }\end{array}$ \\
\hline Juncus cf. effusus L. & $\begin{array}{l}\text { PRIA: } 301+\text { RIA: 301c 311c GIA: 301c VA: } \\
\text { 301c 304c 334c }\end{array}$ & $\begin{array}{l}\text { Juncus effusus recorded from BI: VIIb } \\
\text { (Godwin 1975) NL: } 2800 \pm 50 \text { B.P. (Geel et } \\
\text { al. 1983) BRD: 12-8 B.C. (Kučan 1981) }\end{array}$ \\
\hline Juncus filiformis L. & VA: $103 c$ & \\
\hline $\begin{array}{l}\text { Juncus gerardi Loisel in } \\
\text { Desv. }\end{array}$ & $\begin{array}{l}\text { PRIA: 301c RIA: 301c } 311 \text { c GIA: 301c VA: } \\
\text { 301c 307c 320+ }\end{array}$ & $\begin{array}{l}\text { NL: } 3400-3300 \text { B.C. (Zeist \& Palfenier- } \\
\text { Vegter 1981) }\end{array}$ \\
\hline $\begin{array}{l}\text { Juncus cf. gerardi Loisel in } \\
\text { Desv. }\end{array}$ & VA: $304 c 334 c$ & \\
\hline $\begin{array}{l}\text { Juncus cf. inflexus L. (J. } \\
\text { glaucus Ehrh.) }\end{array}$ & VA: $304+334+$ & $\begin{array}{l}\text { Juncus inflexus recorded from BI: VIIb } \\
\text { (Godwin 1975) NL: } 1680 \pm 40 \text { B.P. (Geel et } \\
\text { al. 1983) }\end{array}$ \\
\hline Juncus squarrosus $\mathrm{L}$. & PRIA: 301+ RIA: 301c GIA: 301r VA: 301+ & \\
\hline Juncus subnodulosus Schrank & VA: $304+334 c$ & $\begin{array}{l}\text { BI: 2nd century A.D. (Wilson 1979), 10th } \\
\text { century A.D. (Wilson 1975) NL: } 600-400 \\
\text { B.C. (Zeist 1974) }\end{array}$ \\
\hline Juncus sp. & $\begin{array}{l}\text { VIII: } 127 \text { r RIA: } 139 \mathrm{c} 301 \mathrm{c} \text { GIA: } 242 \mathrm{c} 243 \mathrm{c} \\
\text { 244c 301+ VA: } 172 \mathrm{c} 301+304 \mathrm{c} 334 \mathrm{c} \text { EMA: } \\
\text { 172c } 245 \mathrm{c} 428 \mathrm{c} 429 \mathrm{c} 430 \mathrm{c} 431 \mathrm{c} \text { LMA: } 245 \mathrm{c} \\
427 \mathrm{c} 428 \mathrm{c} 429 \mathrm{c} 430 \mathrm{c} 521+\mathrm{u}\end{array}$ & $\begin{array}{l}\text { BI: I-VIII (Godwin 1975) NL: } 2800 \pm 50 \\
\text { B.P. (Geel et al. 1983) BRD: IX 'Overbeck' } \\
\text { (Schwaar 1976) }\end{array}$ \\
\hline Juniperus communis L. & $\begin{array}{l}\text { II: } 508+510 \text { c } 511 \text { c } 720 \text { r } 827+\text { III: } 508 \text { r } 704 \text { ru } \\
720 \text { r } 827+\text { IX: } 826+\end{array}$ & BI: I-VI, VIIb-VIII (Godwin 1975) \\
\hline $\begin{array}{l}\text { Knautia arvensis (L.) } \\
\text { Coulter }\end{array}$ & $\begin{array}{l}\text { VIII: } 214 \text { r VA: } 110+\text { EMA: } 521+\text { u LMA: } \\
\text { 218c } 522 r\end{array}$ & \\
\hline Lactuca sativa $\mathrm{L}$. & LMA: $241+$ & \\
\hline Lamium album L. & RIA: 306r LMA: 1025+ & NL: 1000 B.C. (Pals 1977) \\
\hline Lamium amplexicaule $\mathrm{L}$. & LMA: $504 r$ & $\begin{array}{l}\text { BRD: 1st century A.D. (Knörzer 1970), } \\
\text { 15th century A.D. (Knörzer 1975) DDR: } \\
\text { 7th-9th century A.D. (Lange 1976) S: } 1500 \\
\text { A.D. (Griffin 1982) }\end{array}$ \\
\hline
\end{tabular}


Table 2 continued

\begin{tabular}{|c|c|c|}
\hline Species & $\begin{array}{l}\text { Period, site number (cf. Table 1) and number } \\
\text { of macrofossils reported }\end{array}$ & $\begin{array}{l}\text { Reports on finds from neighbour countries. } \\
\text { Pollen zones and other periods are presented } \\
\text { as published by the authors cited }\end{array}$ \\
\hline Lamium purpureum L. & $\begin{array}{l}\text { RIA: } 311+\text { VA: } 307+334+\text { EMA: } 992 r \\
\text { LMA: } 552 \text { r } 1025 c\end{array}$ & $\begin{array}{l}\text { BI: Roman Period (Godwin 1975) NL: } 200 \\
\text { B.C.-250 A.D. (Zeist 1974) }\end{array}$ \\
\hline Lamium sp. & $\begin{array}{l}\text { VIII: } 968 \text { r RIA: } 301 \text { r GIA: } 301 \text { r VA: } 172+ \\
\text { EMA: } 172+245 \text { c } 427 \text { c } 428+429+521 \text { ru } \\
\text { LMA: } 241 \text { c } 427+428+\end{array}$ & BI: VIIb (Godwin 1975) \\
\hline Lapsana communis $\mathrm{L}$. & $\begin{array}{l}\text { PRIA: } 115 \text { r GIA: } 712 \text { r VA: } 110+172 \text { r } 202 r \\
\text { 334c EMA: } 245+428+429+431+521+u \\
\text { LMA: } 406+427+\end{array}$ & $\begin{array}{l}\text { BI: } 3360 \pm 80 \text { B.P. (Peglar \& Wilson 1978) } \\
\text { NL: 3400-3300 B.C. (Zeist \& Palfenier- } \\
\text { Vegter 1981) BRD: Band Ceramic (Knörzer } \\
\text { 1974a, 1977), 12-8 B.C. (Kučan 1981) P: } \\
\text { Neolithic and later (Gluza \& Wasylikowa } \\
\text { 1977) }\end{array}$ \\
\hline Lapsana cf. communis L. & VIII: $425 \mathrm{r}$ & \\
\hline Lemna sp. & $\begin{array}{l}\text { VA: } 334 c \text { EMA: } 430 \mathrm{c} 431+521 \mathrm{cu} \text { LMA: } 430 \mathrm{c} \\
\text { 521cu }\end{array}$ & $\begin{array}{l}\text { Lemna cf. trisulca recorded from BI: I } \\
\text { (Godwin 1975), Lemna sp. from Iron Age } \\
\text { (Greig 1979a, 1979b) and NL: } 1000 \text { B.C. } \\
\text { (Pals 1977). Lemna minor L. recorded from } \\
\text { BRD: 1st-2nd century A.D. (Körber- } \\
\text { Grohne 1967), Lemna trisulca L. from } \\
\text { Migration Period, 6th-10th century A.D. } \\
\text { (Lange 1979). Lemna sp. from P: Hallstatt } \\
\text { (Gluza \& Wasylikowa 1977) }\end{array}$ \\
\hline Leontodon autumnalis $\mathrm{L}$. & $\begin{array}{l}\text { VIII: } 220+\text { PRIA: } 115 \text { r RIA: } 108 \text { r 301r 306r } \\
\text { 308r 311c VA: } 172+304+307 \text { c 334c EMA: } \\
245+521+\text { u LMA: } 427+521 \text { ru 522+ }\end{array}$ & $\begin{array}{l}\text { BI: II, VI, VIIb-VIII (Godwin 1975) BRD: } \\
\text { Band Ceramic (Knörzer 1967d) }\end{array}$ \\
\hline Lepidium latifolium $\mathrm{L}$. & PRIA: $111 r$ & \\
\hline $\begin{array}{l}\text { Leucanthemum vulgare Lam. } \\
\text { (Chrysanthemum } \\
\text { leucanthemum L.) }\end{array}$ & VA: $103+$ EMA: $521+u$ & $\begin{array}{l}\text { BI: Early Iron Age, Roman Period (Godwin } \\
\text { 1975), c. } 300 \text { A.D. (Wilson 1978) BRD: 1st } \\
\text { century A.D. (Knörzer 1967a, 1967b, 1970), } \\
\text { 2nd century A.D. (Knörzer 1979b), c.200 } \\
\text { A.D. (Körber-Grohne 1979b) DDR: 9th } \\
\text { century A.D. (Lange 1976) P: Early } \\
\text { Medieval (Gluza \& Wasylikowa 1977), 9th- } \\
\text { 10th century A.D. (Wasylikowa 1978) }\end{array}$ \\
\hline Limonium vulgare Miller & VA: $307 \mathrm{c}$ & NL: $600-400$ B.C. (Zeist 1974) \\
\hline Linaria vulgaris Miller & VA: $334 \mathrm{r}$ & $\begin{array}{l}\text { BI: II-III (Godwin 1975) P: Hallstatt and } \\
\text { later (Gluza \& Wasylikowa 1977), 9th-12th } \\
\text { century A.D. (Wasylikowa 1978) }\end{array}$ \\
\hline Linum catharticum $\mathrm{L}$. & $\begin{array}{l}\text { VA: } 202+304 \text { r 334r EMA: } 521+\text { u LMA: } \\
241+427+521+u\end{array}$ & $\begin{array}{l}\text { BI: I-III, VIIb-VIII (Godwin 1975), 2nd } \\
\text { century A.D. (Wilson 1979), c. } 300 \text { A.D. } \\
\text { (Wilson 1978) NL: } 200 \text { B.C.-250 A.D. (Zeist } \\
\text { 1974) BRD: 12-8 B.C. (Kučan 1981), 1st } \\
\text { century A.D. (Knörzer 1970), c. } 200 \text { A.D. } \\
\text { (Körber-Grohne 1979b) }\end{array}$ \\
\hline Linum usitatissimum $\mathbf{L}$. & $\begin{array}{l}\text { VIII: 543c PRIA: 114c } 115+201 \mathrm{r} \text { RIA: } 107 \mathrm{r} \\
\text { 108c } 117 \mathrm{r} 118 \mathrm{r} 120 \mathrm{r} 124 \mathrm{r} 125+128 \mathrm{r} 139 \mathrm{c} 140+ \\
\text { 146r 151c } 173 \mathrm{c} 174 \mathrm{c} 210 \mathrm{r} 301+303 \mathrm{c} 306 \mathrm{c} 308 \mathrm{c} \\
\text { 311 } 316 \mathrm{c} 707+708+\text { GIA: } 205 \mathrm{r} 301 \mathrm{r} 1087 \mathrm{r} \\
\text { VA: } 172 \mathrm{r} 301 \mathrm{r} 304 \mathrm{c} 307 \mathrm{c} 312 \mathrm{c} 320 \mathrm{c} 334 \mathrm{c} \text { EMA: } \\
\text { 172+ 245+428c } 429+430+431+521 \mathrm{cu} \\
\text { LMA: } 218 \mathrm{r} 241+406+427+430+521+\mathrm{u} \\
522 \mathrm{c}\end{array}$ & $\begin{array}{l}\text { BI: VIIb-VIII (Godwin 1975) NL: } 1000 \\
\text { B.C. (Pals 1977) BRD: Band Ceramic } \\
\text { (Knörzer 1974a, 1977). Finds of Linum } \\
\text { summarized by Willerding (1970) P: } \\
\text { Neolithic (Gluza \& Wasylikowa 1977) }\end{array}$ \\
\hline Linum cf. usitatissimum L. & IX: 993r RIA: 993r 994ru & \\
\hline Lolium perenne $\mathrm{L}$. & $\begin{array}{l}\text { PRIA: } 115+\text { RIA: } 139+708 \text { r GIA: } 712+\text { VA: } \\
\text { 307c }\end{array}$ & NL: 500 B.C.-200 A.D. (Zeist 1974) \\
\hline Lolium cf. perenne $\mathbf{L}$. & VA: 985r EMA: $992+$ & \\
\hline
\end{tabular}


Table 2 continued

\begin{tabular}{|c|c|c|}
\hline Species & $\begin{array}{l}\text { Period, site number (cf. Table 1) and number } \\
\text { of macrofossils reported }\end{array}$ & $\begin{array}{l}\text { Reports on finds from neighbour countries. } \\
\text { Pollen zones and other periods are presented } \\
\text { as published by the authors cited }\end{array}$ \\
\hline Lolium cf. remotum Schrank & PRIA: $115+$ & \\
\hline Lolium temulentum $\mathrm{L}$. & VA: $304 \mathrm{r} 334 \mathrm{r}$ & $\begin{array}{l}\text { P: Hallstatt and later (Gluza \& Wasylikowa } \\
\text { 1977) }\end{array}$ \\
\hline Lolium cf. temulentum L. & VIII: $1034 r$ 1065r & \\
\hline Lolium sp. & VIII: 127 r GIA: $242 r$ & \\
\hline Lotus corniculatus L. & RIA: $316 c$ VA: $304 r$ & \\
\hline $\begin{array}{l}\text { Lotus corniculatus L. et L. } \\
\text { tenuis Waldst. \& Kit. ex } \\
\text { Willd. }\end{array}$ & VA: $202 r$ & \\
\hline $\begin{array}{l}\text { Lotus uliginosus } \\
\text { Schkuhr }\end{array}$ & VA: $304 r 334 r$ & \\
\hline $\begin{array}{l}\text { Luzula campestris (L.) DC. } \\
\text { in Lam. \& DC. }\end{array}$ & $\begin{array}{l}\text { VIII: 207r 219r 229r 230c PRIA: } 115 \text { r RIA: } \\
\text { 101+ 708r GIA: 712r VA: 334c }\end{array}$ & \\
\hline $\begin{array}{l}\text { Luzula cf. campestris (L.) } \\
\text { DC. in Lam. \& DC. }\end{array}$ & PRIA: $301+$ RIA: $301+$ VA: $301 \mathrm{r}$ & \\
\hline Luzula sp. & VIII: 609 r VA: $202 r$ & \\
\hline $\begin{array}{l}\text { Lychnis flos-cuculi L. } \\
\text { (Coronaria flos-cuculi (L.) } \\
\text { A. Braun) }\end{array}$ & $\begin{array}{l}\text { VI, VII: 555r VIII: 323c 331r IX: 323r 327r } \\
\text { 328+ 331r 332+ RIA: 101+ 301r 311c GIA: } \\
\text { 242r 244c VA: 172r 202+ 301r 304c 307c 334c } \\
\text { EMA: 428r 430+ LMA: } 241+427+429+ \\
\text { 521ru }\end{array}$ & $\begin{array}{l}\text { BI: I-VIII (Godwin 1975) NL: } 3400-3300 \\
\text { B.C. (Zeist \& Palfenier-Vegter 1981) }\end{array}$ \\
\hline Lycopus europaeus L. & $\begin{array}{l}\text { IV: 330r V: 711ru VI, VII: 422ru 944r } \\
\text { VIII: 322r 323+ 513ru 531r 603ru } \\
\text { IX: 332+ 513+ 711r PRIA: 301+ RIA: } 101+ \\
\text { 301c 306r 311+ GIA: 306r 553r VA: 202r } \\
\text { 301r 304+ 307+ 334c EMA: } 245+428+430 \mathrm{c} \\
\text { 431+ 521ru LMA: } 217+\mathrm{u} 218 \mathrm{r} 241+430+ \\
521+\mathrm{u}\end{array}$ & \\
\hline Lysimachia thyrsiflora $\mathrm{L}$. & IX: $502+$ & \\
\hline Lysimachia sp. & VIII: 531r EMA: 521ru LMA: 521ru & BI: VIlb (Godwin 1975) \\
\hline Lythrum salicaria L. & VIII: 531r VA: $334 c$ & $\begin{array}{l}\text { BI: VIIa (Godwin 1975) NL: } 2860 \pm 30 \text { B.P. } \\
\text { (Pals et al. 1980) }\end{array}$ \\
\hline $\begin{array}{l}\text { Malus sylvestris Miller (M. } \\
\text { communis Mill., M. } \\
\text { domestica Borkh, Pyrus } \\
\text { malus) }\end{array}$ & $\begin{array}{l}\text { VIII: } 206+208+404 \text { r } 609+\text { VA: } 304+312 c \\
\text { 334c } 985 \text { r EMA: } 245+\text { LMA: } 406 c\end{array}$ & $\begin{array}{l}\text { BI: VIIb-VIII (Godwin 1975) BRD: } \\
\text { Neolithic (Knörzer 1973) P: Neolithic (Gluza } \\
\text { \& Wasylikowa 1977) }\end{array}$ \\
\hline Malus sp. & 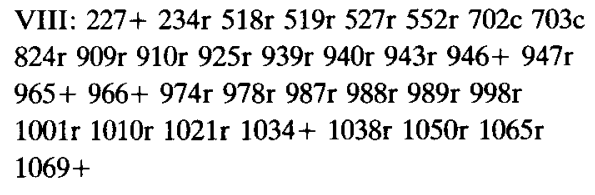 & \\
\hline $\begin{array}{l}\text { Malva pusilla } \mathrm{Sm} \text {. in } \\
\text { Sowerby }\end{array}$ & VIII: $543 r$ VA: $110 r$ & \\
\hline Malva sylvestris $\mathbf{L}$. & PRIA: 301r VA: 110r 334r & $\begin{array}{l}\text { BI: II, VIII (Godwin 1975) BRD: Band } \\
\text { Ceramic (Knörzer 1974a) }\end{array}$ \\
\hline Marrubium vulgare $\mathrm{L}$. & EMA: $521+u$ LMA: $521+u$ & \\
\hline $\begin{array}{l}\text { Matricaria maritima L. } \\
\text { (Tripleurospermum } \\
\text { maritimum (L.) Koch) }\end{array}$ & RIA: 311c VA: 307c & $\begin{array}{l}\text { BRD: 1st-2nd century A.D. (Körber- } \\
\text { Grohne 1967) }\end{array}$ \\
\hline Matricaria cf. maritima L. & VIII: $609+$ & \\
\hline
\end{tabular}




\begin{tabular}{|c|c|c|}
\hline Species & $\begin{array}{l}\text { Period, site number (cf. Table } 1 \text { ) and number } \\
\text { of macrofossils reported }\end{array}$ & $\begin{array}{l}\text { Reports on finds from neighbour countries. } \\
\text { Pollen zones and other periods are presented } \\
\text { as published by the authors cited }\end{array}$ \\
\hline $\begin{array}{l}\text { Matricaria perforata Mérat } \\
\text { (M. inodora L., nom. illeg., } \\
\text { Tripleurospermum inodorum } \\
\text { Schultz Bip.) }\end{array}$ & PRIA: 115r GIA: $301+$ VA: $301+320 c$ & $\begin{array}{l}\text { BI: 3rd century B.C. (Wilson 1978) NL: } \\
\text { 600-400 B.C. (Zeist 1974) }\end{array}$ \\
\hline Matricaria sp. & - LMA: $427+$ & \\
\hline Medicago lupulina $\mathbf{L}$. & RIA: $311+$ VA: $334 c$ & $\begin{array}{l}\text { BI: Late Bronze Age, Iron Age, Roman } \\
\text { Period (Godwin 1975), } 3360 \pm 80 \text { B.P. } \\
\text { (Peglar \& Wilson 1978), c. } 300 \text { A.D. } \\
\text { (Wilson 1978) NL: 1000 B.C. (Pals 1977) } \\
\text { BRD: 12-8 B.C. (Kučan 1981), 1st-2nd } \\
\text { century A.D. (Knörzer 1967a, 1970, 1979b, } \\
\text { Körber-Grohne 1967, 1979b) P: Hallstatt } \\
\text { and later (Gluza \& Wasylikowa 1977) }\end{array}$ \\
\hline Melilotus alba Medicus & VA: $334+$ & \\
\hline Mentha aquatica L. & RIA: $101 \mathrm{r}$ & $\begin{array}{l}\text { BI: IV, VIIb-VIII (Godwin 1975) NL: 3400- } \\
\text { 3300 B.C. (Zeist \& Palfenier-Vegter 1981) }\end{array}$ \\
\hline $\begin{array}{l}\text { Mentha aquatica } \mathrm{L} \text {. et } \mathrm{M} \text {. } \\
\text { arvensis } \mathrm{L} \text {. }\end{array}$ & $\begin{array}{l}\text { RIA: } 311+\text { GIA: } 242 \text { r VA: } 172 \text { r } 202+304+ \\
\text { 307+ 334c EMA: } 172+428+429+431+ \\
\text { LMA: } 427+428 \text { r } 429+430+\end{array}$ & \\
\hline Mentha arvensis $\mathbf{L}$. & RIA: 108c LMA: 564r & $\begin{array}{l}\text { BI: Late 2nd century A.D. (Wilson 1979) } \\
\text { BRD: c. } 200 \text { B.C. (Knörzer 1979a), 1st cen- } \\
\text { tury A.D. (Knörzer 1967a, 1970) NL: } 1000 \\
\text { B.C. (Pals 1977) P: Hallstatt and later (Gluza } \\
\text { \& Wasylikowa 1977) }\end{array}$ \\
\hline Mentha sp. & $\begin{array}{l}\text { VIII: 531r IX: 325r 332+ RIA: } 301 \mathrm{r} \text { 308r } \\
\text { GIA: 301r EMA: } 521+\mathrm{u} \text { LMA: } 521+\mathrm{u}\end{array}$ & BI: I-VI, VIIb-VIII (Godwin 1975) \\
\hline Menyanthes trifoliata L. & 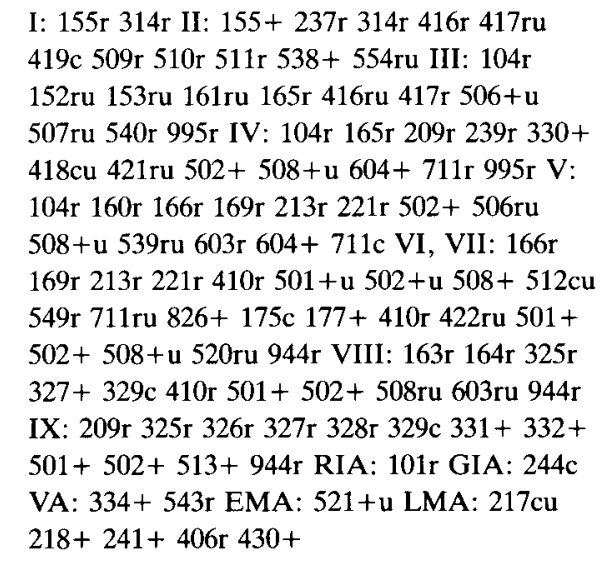 & BI: I-VIII (Godwin 1975) \\
\hline $\begin{array}{l}\text { Moehringia trinervia (L.) } \\
\text { Clairv. (Arenaria trinervia } \\
\text { L.) }\end{array}$ & VI, VII: 528ru RIA: 101+ LMA: $430+$ & $\begin{array}{l}\text { BI: IV/V, VIIa-VIIb (Godwin 1975) NL: } \\
\text { 3400-3300 B.C. (Zeist \& Palfenier-Vegter } \\
\text { 1981) }\end{array}$ \\
\hline $\begin{array}{l}\text { Molinia caerulea (L.) } \\
\text { Moench }\end{array}$ & IX: $501+513 r$ RIA: $301 \mathrm{c}$ VA: $103 \mathrm{r} 334 \mathrm{r}$ & $\begin{array}{l}\text { BI: IV, VIIa-VIII (Godwin 1975), Late 2nd } \\
\text { century A.D. (Wilson 1979) NL: 100-250 } \\
\text { A.D. (Zeist 1974) }\end{array}$ \\
\hline $\begin{array}{l}\text { Montia fontana L. subsp. } \\
\text { chondrosperma (Fenzl) } \\
\text { Walters }\end{array}$ & EMA: $428+430+$ & $\begin{array}{l}\text { BI: VIIa, VIII (Godwin 1975), 3rd century } \\
\text { B.C. (Wilson 1978) }\end{array}$ \\
\hline $\begin{array}{l}\text { Montia fontana L. subsp. } \\
\text { fontana (M. lamprosperma } \\
\text { Cham.) }\end{array}$ & I: 155 r II: 155 r III: $155+$ RIA: $101 \mathrm{c} 139+$ & BI: I-IV (Godwin 1975) \\
\hline
\end{tabular}




\begin{tabular}{|c|c|c|}
\hline Species & $\begin{array}{l}\text { Period, site number (cf. Table 1) and number } \\
\text { of macrofossils reported }\end{array}$ & $\begin{array}{l}\text { Reports on finds from neighbour countries. } \\
\text { Pollen zones and other periods are presented } \\
\text { as published by the authors cited }\end{array}$ \\
\hline Myosotis arvensis (L.) Hill & PRIA: $115 \mathrm{r}$ GIA: $712 \mathrm{r}$ & \\
\hline $\begin{array}{l}\text { Myosotis scorpioides L. (M. } \\
\text { palustris (L.) Hill) }\end{array}$ & GIA: 306 r VA: $304+334+$ & NL: 1000 B.C. (Pals 1977) \\
\hline $\begin{array}{l}\text { Myosotis cf. scorpioides L. } \\
\text { (M. palustris (L.) Hill) }\end{array}$ & IX: $328 \mathrm{r}$ & \\
\hline Myosotis sp. & $\begin{array}{l}\text { GIA: } 244+301 \text { r EMA: } 427+431+\text { LMA: } \\
241+\end{array}$ & \\
\hline $\begin{array}{l}\text { Myosoton aquaticum (L.) } \\
\text { Moench (Malachium } \\
\text { aquaticum (L.) Fries) }\end{array}$ & RIA: $306 \mathrm{r}$ & $\begin{array}{l}\text { BI: IV/V, Roman Period (Godwin 1975) } \\
\text { BRD: } 7200-5500 \text { B.C. (Stalling 1983) }\end{array}$ \\
\hline Myrica gale L. & $\begin{array}{l}\text { VIII: 220r 328c IX: } 164 \text { r 328c RIA: } 602 \mathrm{c} \text { GIA: } \\
\text { 242r } 244+\text { VA: } 103 \text { r } 172 \mathrm{c} 307+312+334+ \\
\text { EMA: } 172 \mathrm{c} 245 \mathrm{c} 428 \mathrm{c} 431+521 \mathrm{cu} \text { LMA: } 218 \mathrm{r} \\
241+245+406+427+428+521+\mathrm{u} 522 \mathrm{c}\end{array}$ & $\begin{array}{l}\text { BI: IV, VIIa-VIII (Godwin 1975) NL: } 2510 \\
\pm 35 \text { B.P. (Geel et al. 1983) }\end{array}$ \\
\hline $\begin{array}{l}\text { Myriophyllum alterniflorum } \\
\text { DC. in Lam. \& DC. }\end{array}$ & III: $158+\mathrm{u} 161+\mathrm{u}$ & BI: I-VIII (Godwin 1975) \\
\hline Myriophyllum spicatum L. & $\begin{array}{l}\text { I: } 319 r \text { II: } 319 r \text { 416ru 417ru 419+ 705r 720r } \\
\text { III: 319r 417ru 426ru 503ru 704ru IV: 238ru } \\
\text { 418r V: 238ru VIII: 603ru 1036r }\end{array}$ & BI: I-IV, VI, VIIb-VIII (Godwin 1975) \\
\hline $\begin{array}{l}\text { Myriophyllum verticillatum } \\
\text { L. }\end{array}$ & $\begin{array}{l}\text { III: } 104+\text { IV: } 104+\text { V: } 562 \text { r VI, VII: 562r VA: } \\
334 \mathrm{r}\end{array}$ & BI: II, IV, VIIb (Godwin 1975) \\
\hline Myriophyllum sp. & $\begin{array}{l}\text { II: } 508 \mathrm{c} 513 \mathrm{c} \text { III: } 506+\mathrm{u} 508+995 \mathrm{r} \text { IV: } 711 \mathrm{r} \\
\text { 995r EMA: } 521 \mathrm{ru}\end{array}$ & \\
\hline $\begin{array}{l}\text { Najas flexilis (Willd.) Rostk. } \\
\text { \& W. L. E. Schmidt }\end{array}$ & $\begin{array}{l}\text { V: } 815 \text { r 816r 934r 1082r VI, VII: 820r 820r } \\
821 r\end{array}$ & \\
\hline Najas marina $L$. & $\begin{array}{l}\text { IV: } 104+407 \mathrm{r} 420 \mathrm{r} \text { V: } 407 \mathrm{c} 420 \mathrm{r} 539 \mathrm{cu} 549 \mathrm{r} \\
555+562 \mathrm{r} 603 \mathrm{ru} 944 \mathrm{r} 952 \mathrm{r} \text { VI, VII: } 407 \mathrm{c} 410 \mathrm{c} \\
420 \mathrm{c} 549+556+562 \mathrm{r} 603 \mathrm{ru} 944 \mathrm{r} 407 \mathrm{r} 410 \mathrm{c} \\
420 \mathrm{r} 422 \mathrm{cu} 508 \mathrm{ru} 513+\mathrm{u} 551 \mathrm{cu} 556 \mathrm{c} 603 \mathrm{ru} \\
818+826+944 \mathrm{r} 1036 \mathrm{r} \text { VIII: } 410 \mathrm{c} 513+\mathrm{u} 543 \mathrm{r} \\
551 \mathrm{c} 556 \mathrm{r} 603 \mathrm{ru} 818+826+1036 \mathrm{r} \text { IX: } 513+ \\
\text { 556r } 826+\text { GIA: 553c LMA: } 217+\mathrm{u}\end{array}$ & \\
\hline Najas sp. & V: 944r VI, VII: 944r & \\
\hline Neslia paniculata (L.) Desv. & $\begin{array}{l}\text { VA: } 334+985+\text { EMA: } 428+429+521+u \\
709 \text { r LMA: } 427+428+521+u\end{array}$ & $\begin{array}{l}\text { BRD: c. 10th century A.D. (Willerding } \\
\text { 1973) P: Early Medieval Period (Gluza \& } \\
\text { Wasylikowa 1977), 9th-12th century A.D. } \\
\text { (Wasylikowa 1978), 11th century A.D. } \\
\text { (Kosina 1978) }\end{array}$ \\
\hline $\begin{array}{l}\text { Nuphar lutea (L.) Sibth. \& } \\
\text { Sm. (N. luteum) }\end{array}$ & $\begin{array}{l}\text { IV: 238ru 604c V: 238ru 534r 603ru 944r VI, } \\
\text { VII: 512cu 529r 530c 534r 535r 549c 550c } \\
\text { 603ru 944r 422cu 502+508+u 513ru 603ru } \\
\text { 906r 944r VIII: 502+513r 531c 603ru 906r } \\
\text { 1036r IX: 502+ 513c } 906+1091+\text { LMA: } \\
\text { 217cu }\end{array}$ & BI: I-II, IV-VIII (Godwin 1975) \\
\hline Nuphar pumila (Timm) DC. & II: $419+$ & \\
\hline Nuphar sp. & $\begin{array}{l}\text { V: } 952 r \text { VI, VII: } 905 \text { r 905r VIII: 323r 905r IX: } \\
\text { 905r }\end{array}$ & \\
\hline $\begin{array}{l}\text { Nymphaea alba } \mathbf{L} \text {. } \\
\text { (to be continued) }\end{array}$ & $\begin{array}{l}\text { III: } 104+161+\text { u } 995 \text { r IV: } 104+238 \text { ru } 502+ \\
513+944 \text { r } 995 \text { r V: } 238 r u 407 \text { r } 502+513+529 \mathrm{c} \\
534 \text { r } 535 \text { r } 539+\text { u } 549+555+\end{array}$ & BI: I-VIII (Godwin 1975) \\
\hline
\end{tabular}


Table 2 continued

\begin{tabular}{|c|c|c|}
\hline Species & $\begin{array}{l}\text { Period, site number (cf. Table 1) and number } \\
\text { of macrofossils reported }\end{array}$ & $\begin{array}{l}\text { Reports on finds from neighbour countries. } \\
\text { Pollen zones and other periods are presented } \\
\text { as published by the authors cited }\end{array}$ \\
\hline $\begin{array}{l}\text { Nymphaea alba L. } \\
\text { (continued) }\end{array}$ & $\begin{array}{l}\text { 603ru 944r 952r VI, VII: } 170+410 \mathrm{r} 502+\mathrm{u} \\
512 \mathrm{cu} 513+529 \mathrm{r} 530 \mathrm{c} 534 \mathrm{r} 535 \mathrm{r} 537 \mathrm{r} 549 \mathrm{c} 550 \mathrm{r} \\
603 \mathrm{ru} 944 \mathrm{r} 164 \mathrm{r} 410 \mathrm{r} \text { 422cu 502+ 508+u } \\
513+\mathrm{u} 603 \mathrm{r} 818 \mathrm{c} 944 \mathrm{r} 1036 \mathrm{r} \text { VIII: 410r } 502+ \\
513+\mathrm{u} 603 \mathrm{ru} 1036 \mathrm{r} \text { IX: } 513 \mathrm{c} 1091+\text { LMA: } \\
217 \mathrm{cu} 406 \mathrm{r} 430 \mathrm{c}\end{array}$ & \\
\hline Nymphaea sp. & $\begin{array}{l}\text { III: } 905 \text { r IV: } 952 \text { r V: 420r } 826+905 \text { r VI, VII: } \\
\text { 420r } 826+905+551 r u \text { 826+ 905r 906r VIII: } \\
\text { 326r 551r 905r 906r IX: 332r 905+ 906r }\end{array}$ & \\
\hline Odontites verna (Bellardi) & RIA: $311 \mathrm{c}$ VA: $103 \mathrm{c} 304 \mathrm{c} 307 \mathrm{c} 334 \mathrm{c} 985 \mathrm{r}$ & BI: Late Weichselian (Godwin 1975), Late \\
\hline $\begin{array}{l}\text { Dumort. (O. rubra (Besser) } \\
\text { Gilib.) }\end{array}$ & LMA: $245+$ & $\begin{array}{l}\text { 2nd century A.D. (Wilson 1979) NL: } 1680 \\
\pm 40 \text { B.P. (Geel et al. 1983) BRD: 12-8 B.C. } \\
\text { (Kučan 1981) }\end{array}$ \\
\hline cf. Odontites sp. & $\begin{array}{l}\text { PRIA: 301r RIA: 301r GIA: 301+ VA: } \\
\text { 301+ }\end{array}$ & \\
\hline $\begin{array}{l}\text { Oenanthe aquatica (L.) } \\
\text { Poiret in Lam. (O. } \\
\text { phellandrium Lam.) }\end{array}$ & $\begin{array}{l}\text { II: } 416 \mathrm{cu} 417 \mathrm{ru} \text { VI, VII: 555+ 422ru RIA: } \\
\text { 311c VA: 304c 307c 334c EMA: 521+u LMA: } \\
\text { 521cu }\end{array}$ & \\
\hline Oenanthe fistulosa L. & VA: $334 c$ & $\begin{array}{l}\text { BI: Roman Period (Godwin 1975) NL: } 2690 \\
\pm 60 \text { B.P. (Geel et al. 1983) }\end{array}$ \\
\hline $\begin{array}{l}\text { Oenanthe lachenalii C. C. } \\
\text { Gmelin }\end{array}$ & RIA: $301 \mathrm{r} 306 \mathrm{r} 311+\mathrm{VA}: 307 \mathrm{c} 334 \mathrm{c}$ & $\begin{array}{l}\text { BI: Late Weichselian, Roman Period } \\
\text { (Godwin 1975) NL: 600-400 B.C. (Zeist } \\
\text { 1974) BRD: 1st-2nd century A.D. (Körber- } \\
\text { Grohne 1967) }\end{array}$ \\
\hline Oenanthe sp. & VA: $334 c$ & \\
\hline Origanum vulgare $\mathrm{L}$. & EMA: $431+$ & $\begin{array}{l}\text { BI: II, VIIa-VIII (Godwin 1975) BRD: 1st } \\
\text { century A.D. (Knörzer 1970) P: 13th } \\
\text { century A.D. (Wieserowa 1979) }\end{array}$ \\
\hline Ornithopus perpusillus $\mathbf{L}$. & VA: $304 \mathrm{c} \mathrm{334c}$ & \\
\hline Oryza sativa $\mathrm{L}$. & LMA: $957 \mathrm{c}$ & BRD: 1st century A.D. (Knörzer 1970) \\
\hline Oxalis acetosella $\mathbf{L}$. & IX: 501r 513+ EMA: 521ru & $\begin{array}{l}\text { BRD: } 5500-3000 \text { B.C. (Stalling 1983), c. } \\
1209 \text { A.D. (Lynch \& Paap 1982) N: } 1120 \\
\text { A.D. (Tallantire 1979) }\end{array}$ \\
\hline Panicum miliaceum L. & $\begin{array}{l}\text { VIII: 425c 541r 606r 607r 702r 703r 922+ } \\
\text { 923+ 968r 969r 975r PRIA: } 141+\text { RIA: } 311 \mathrm{c} \\
\text { VA: } 312 \mathrm{c} 334 \mathrm{c} \text { EMA: } 521+\mathrm{u}\end{array}$ & $\begin{array}{l}\text { NL: } 1155 \pm 65 \text { B.C. (Zeist 1968) BRD: } \\
\text { Bronze Age (Behre 1982) P: Neolithic, } \\
\text { Bronze Age (Gluza \& Wasylikowa 1977) }\end{array}$ \\
\hline Panicum sp. & PRIA: 201r & \\
\hline Papaver argemone L. & LMA: $427 \mathrm{c}$ & $\begin{array}{l}\text { BI: Roman Period (Godwin 1975), c. } 300 \\
\text { A.D. (Wilson 1978) BRD: 1st century A.D. } \\
\text { (Knörzer 1967b, 1970), 11th-12th century } \\
\text { A.D. (Knörzer 1968), 13th century (Lynch } \\
\text { \& Paap 1982), Late Middle Ages (Willerding } \\
\text { 1978b) P: 13th century A.D. (Wieserowa } \\
\text { 1979) N: Late Viking (Tallantire 1979) }\end{array}$ \\
\hline $\begin{array}{l}\text { Papaver dubium L. et } \mathrm{P} \text {. } \\
\text { rhoeas } \mathrm{L} \text {. }\end{array}$ & VA: $334 r$ & \\
\hline Papaver somniferum L. & $\begin{array}{l}\text { PRIA: 178r EMA: 428r LMA: } 241+406+ \\
427+\end{array}$ & $\begin{array}{l}\text { BI: Early Iron Age and later (Godwin 1975) } \\
\text { BRD: Band Ceramic (Knörzer 1971b), } \\
\text { Hallstatt (Knörzer 1971c) P: Neolithic, } \\
\text { Hallstatt and later (Gluza \& Wasylikowa } \\
\text { 1977) }\end{array}$ \\
\hline Papaver sp. & EMA: $245+521$ ru LMA: 427+ 521ru & \\
\hline
\end{tabular}




\begin{tabular}{|c|c|c|}
\hline Species & $\begin{array}{l}\text { Period, site number (cf. Table 1) and number } \\
\text { of macrofossils reported }\end{array}$ & $\begin{array}{l}\text { Reports on finds from neighbour countries. } \\
\text { Pollen zones and other periods are presented } \\
\text { as published by the authors cited }\end{array}$ \\
\hline Pedicularis palustris $\mathrm{L}$. & $\begin{array}{l}\text { GIA: } 244 \text { c VA: } 304+307+334+\text { LMA: } 218+ \\
522 \text { r }\end{array}$ & $\begin{array}{l}\text { BI: I, III-IV, Roman Period (Godwin 1975), } \\
\text { 1st-early 2nd century A.D. (Kenward \& } \\
\text { Williams 1979) NL: } 1870 \pm 60 \text { B.P. (Geel et } \\
\text { al. 1983) }\end{array}$ \\
\hline $\begin{array}{l}\text { Petasites hybridus (L.) P. } \\
\text { Gaertner, B. Meyer \& } \\
\text { Scherb. }\end{array}$ & VA: $202 r$ & \\
\hline $\begin{array}{l}\text { Peucedanum palustre (L.) } \\
\text { Moench }\end{array}$ & $\begin{array}{l}\text { V: 539ru VI, VII: 422ru 508cu VIII: 508ru IX: } \\
\text { 501+ 513c VA: 334r LMA: } 217+u\end{array}$ & \\
\hline Phalaris arundinacea $\mathrm{L}$. & RIA: $301 \mathrm{c} 311+$ & $\begin{array}{l}\text { NL: } 370 \pm 70 \text { B.C. (Zeist 1974) BRD: 12-8 } \\
\text { B.C. (Kučan 1981), 1st-2nd century A.D. } \\
\text { (Körber-Grohne 1967, 1979b) }\end{array}$ \\
\hline $\begin{array}{l}\text { Phleum pratense L. subsp. } \\
\text { bertolonii (DC.) Bornm. (P. } \\
\text { nodosum L., P. bertolonii } \\
\text { DC.) }\end{array}$ & $\begin{array}{l}\text { PRIA: } 111+\text { RIA: 708r GIA: 205r 712c VA: } \\
\text { 110r }\end{array}$ & \\
\hline Phleum cf. pratense L. & VIII: $208 \mathrm{c}$ & \\
\hline Phleum sp. & VIII: 405c 425+ PRIA: 115r RIA: 139+ & $\begin{array}{l}\text { BRD: Band Ceramic (Knörzer 1967d, 1974a, } \\
\text { 1977) }\end{array}$ \\
\hline $\begin{array}{l}\text { Phragmites australis (Cav.) } \\
\text { Trin. ex Steudel (P. } \\
\text { communis Trin., Arundo } \\
\text { phragmites L.) }\end{array}$ & 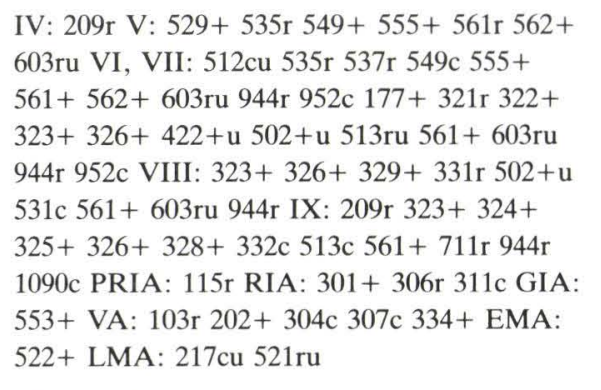 & \\
\hline Pimpinella sp. & EMA: $521 \mathrm{ru}$ & $\begin{array}{l}\text { BRD: 12-8 B.C. (Kučan 1981). Pimpinella } \\
\text { major recorded from P: Hallstatt (Gluza \& } \\
\text { Wasylikowa 1977). Pimpinella cf. major } \\
\text { recorded from P: } 11 \text { th-12th century A.D. } \\
\text { (Wasylikowa 1978), and from N: } 1150-1225 \\
\text { A.D. (Griffin 1979a). Pimpinella saxifraga } \\
\text { recorded from P: Early Medieval (Gluza \& } \\
\text { Wasylikowa 1977) }\end{array}$ \\
\hline Pinus pinea $\mathbf{L}$. & RIA:412r & $\begin{array}{l}\text { BI: Roman Period (Godwin 1975, Willcox } \\
\text { 1977) }\end{array}$ \\
\hline Pinus sylvestris $\mathrm{L}$. & $\begin{array}{l}\text { III: } 827+\text { IV: } 238+\text { u } 502+508+513 \mathrm{c} 827+ \\
\text { V: } 238+\text { u } 502+508+\text { u } 513 \mathrm{c} 530 \mathrm{c} 539 \mathrm{cu} 826+ \\
944 \mathrm{r} \text { VI, VII: } 410 \mathrm{r} 501 \mathrm{cu} 502+\mathrm{u} 508+512 \mathrm{ru} \\
513 \mathrm{c} 826+944 \mathrm{r} 159 \mathrm{r} 410 \mathrm{r} 422 \mathrm{ru} 508 \mathrm{ru} 513 \mathrm{cu} \\
826+944 \mathrm{r} \text { VIII: } 410 \mathrm{r} 513 \mathrm{cu} 826+944 \mathrm{r} \text { IX: } \\
513 \mathrm{r} 944 \mathrm{r}\end{array}$ & $\begin{array}{l}\text { BI: II-VIII (Godwin 1975) P: Palaeolithic, } \\
\text { Mesolithic, Neolithic, Bronze Age, Iron Age } \\
\text { (Gluza \& Wasylikowa 1977) }\end{array}$ \\
\hline Pinus cf. sylvestris L. & VIII: $176+$ & \\
\hline Pinus sp. & $\mathrm{V}: 235 \mathrm{cu}$ & \\
\hline Pisum sativum L. & RIA: $108+125+$ VA: $202+$ LMA: $957 \mathrm{c}$ & $\begin{array}{l}\text { BI: Roman Period (Godwin 1975, Willcox } \\
\text { 1977) BRD: Band Ceramic (Knörzer 1967d, } \\
\text { 1974a, 1977), c. } 200 \text { B.C. (Knörzer 1979a), } \\
\text { 1st century A.D. (Knörzer 1967a, 1967c, } \\
\text { 1970) DDR: 3rd-4th century A.D. } \\
\text { (Schiemann 1957) P: Neolithic, Hallstatt and } \\
\text { later (Gluza \& Wasylikowa 1977) }\end{array}$ \\
\hline $\begin{array}{l}\text { Pisum sativum L. subsp. } \\
\text { sativum (P. arvense L.) }\end{array}$ & VA: 985c LMA: $809 \mathrm{r}$ & \\
\hline
\end{tabular}


Table 2 continued

\begin{tabular}{|c|c|c|}
\hline Species & $\begin{array}{l}\text { Period, site number (cf. Table 1) and number } \\
\text { of macrofossils reported }\end{array}$ & $\begin{array}{l}\text { Reports on finds from neighbour countries. } \\
\text { Pollen zones and other periods are presented } \\
\text { as published by the authors cited }\end{array}$ \\
\hline cf. Pisum sativum $\mathbf{L}$. & VIII: $409 \mathrm{r}$ & \\
\hline Pisum sp. & VIII: $925 r$ & \\
\hline Plantago coronopus L. & RIA: $311+$ VA: $307 \mathrm{c}$ & \\
\hline Plantago lanceolata L. & $\begin{array}{l}\text { VIII: } 220+411+702 \text { r PRIA: 111r 114r } 115+ \\
\text { 301r RIA: 301r 308+ 315r 708r GIA: 301r } \\
\text { 712r VA: } 103 \mathrm{r} 301+334+985 \text { r EMA: } 428 \mathrm{r} \\
\text { LMA: } 241+\end{array}$ & $\begin{array}{l}\text { BI: VIIb-VIII (Godwin 1975) NL: } 2005 \pm 50 \\
\text { B.C. (Zeist 1968) }\end{array}$ \\
\hline Plantago major $\mathrm{L}$. & $\begin{array}{l}\text { PRIA: } 115+\text { RIA: } 301+311 \mathrm{c} \text { GIA: } 301+ \\
712+\text { VA: } 301+304 \mathrm{c} 307 \mathrm{c} 334 \mathrm{c} 985 \mathrm{r}\end{array}$ & $\begin{array}{l}\text { BI: } 3360 \pm 80 \text { B.P. (Peglar \& Wilson 1978) } \\
\text { NL: } 3400-3300 \text { B.C. (Zeist \& Palfenier- } \\
\text { Vegter 1981) BRD: } 12-8 \text { B.C. (Kučan 1981) }\end{array}$ \\
\hline Plantago maritima L. & RIA: $311 \mathrm{c}$ VA: $307 \mathrm{c} 320+$ & $\begin{array}{l}\text { NL: } 600-400 \text { B.C. (Zeist 1974) BRD: 1st- } \\
\text { 2nd century A.D. (Körber-Grohne 1967) }\end{array}$ \\
\hline Plantago sp. & PRIA: 201r RIA: 311c VA: 304r 307c 334+ & \\
\hline Poa annua $\mathbf{L}$. & $\begin{array}{l}\text { PRIA: 301r RIA: 301c 311c GIA: 301r VA: } \\
\text { 301+ 304c 307+ 334c EMA: 428r }\end{array}$ & $\begin{array}{l}\text { NL: } 370 \pm 70 \text { B.C. (Zeist 1974) BRD: } 12-8 \\
\text { B.C. (Kučan 1981) }\end{array}$ \\
\hline Poa nemoralis $\mathrm{L}$. & PRIA: $115 r$ & \\
\hline Poa cf. palustris L. & VA: $304 \mathrm{c}$ & $\begin{array}{l}\text { Poa palustris recorded from BI: 2nd century } \\
\text { A.D. (Wilson 1979), and from BRD: 1st- } \\
\text { 2nd century A.D. (Körber-Grohne 1967, } \\
\text { 1979b) }\end{array}$ \\
\hline $\begin{array}{l}\text { Poa pratensis } \mathrm{L} \text {. et } \mathrm{P} \text {. trivialis } \\
\mathrm{L} \text {. }\end{array}$ & RIA: $311 \mathrm{c} 315+$ VA: $304 \mathrm{c} 307 \mathrm{c} 320 \mathrm{c} 334 \mathrm{c}$ & $\begin{array}{l}\text { BI: Late Weichselian (Godwin 1975). Poa } \\
\text { pratensis recorded from Late 2nd century } \\
\text { A.D. (Wilson 1979). P. pratensis et P. } \\
\text { trivialis recorded from NL: } 600-400 \text { B.C. } \\
\text { (Zeist 1974) BRD: 12-8 B.C. (Kučan 1981), } \\
\text { 1st-2nd century A.D. (Körber-Grohne 1967, } \\
\text { 1979b) }\end{array}$ \\
\hline Poa trivialis $\mathrm{L}$. & GIA: $243+$ EMA: $428 \mathrm{r}$ & \\
\hline Poa sp. & $\begin{array}{l}\text { VIII: } 127+405+\text { PRIA: } 111 \text { r 115r RIA: } 139+ \\
\text { 301c GIA: } 712 \text { r }\end{array}$ & $\begin{array}{l}\text { BI: I (Godwin 1975) NL: } 3400-3300 \text { B.C. } \\
\text { (Zeist \& Palfenier-Vegter 1981) BRD: Band } \\
\text { Ceramic (Knörzer 1974a) }\end{array}$ \\
\hline Polygala vulgaris $\mathrm{L}$. & EMA: 521ru & N: 400-550 A.D. (Griffin 1981) \\
\hline Polygonum amphibium $\mathrm{L}$. & LMA: $521+\mathrm{u}$ & $\begin{array}{l}\text { BI: I-III, VIII (Godwin 1975) BRD: } 1225- \\
1265 \text { A.D. (Körber-Grohne 1979a) DDR: } \\
\text { 7th-9th century A.D. (Lange 1976) P: 11th } \\
\text { century A.D. (Kosina 1978) }\end{array}$ \\
\hline Polygonum aviculare L. & $\begin{array}{l}\text { VI, VII: 159r VIII: 116r 127r 214+220+ 230r } \\
405+425+543+609 \mathrm{r} \text { PRIA: } 109+115 \mathrm{r} 301 \mathrm{c} \\
\text { RIA: } 107+108 \mathrm{c} 139 \mathrm{r} \text { 301c 302r 306c 308c 311c } \\
\text { 315r 708+ GIA: } 135+205 \mathrm{r} 242 \mathrm{c} 243+244+ \\
\text { 301c 306r 712+ VA: } 103+110 \mathrm{r} 172 \mathrm{c} 202+ \\
\text { 301c 304c 307c 320+ 334c 985r EMA: } 172 \mathrm{c} \\
245 \mathrm{c} 427+428 \mathrm{c} 429 \mathrm{c} 430+431+521 \mathrm{cu} \text { LMA: } \\
218+241+245+406 \mathrm{r} 427 \mathrm{c} 428 \mathrm{r} 430+504+ \\
521 \mathrm{cu} 522+809 \mathrm{r}\end{array}$ & $\begin{array}{l}\text { NL: } 3400-3300 \text { B.C. (Zeist \& Palfenier- } \\
\text { Vegter 1981) }\end{array}$ \\
\hline Polygonum hydropiper L. & $\begin{array}{l}\text { VIII: } 543+\text { RIA: } 101 \mathrm{c} 306+\text { GIA: } 242+244 \mathrm{c} \\
\text { 712r VA: } 172 \mathrm{c} 304 \mathrm{c} 334 \mathrm{c} \text { EMA: } 172 \mathrm{r} 245+ \\
429 \mathrm{c} 431+\text { LMA: } 218 \mathrm{r} 241+245+427 \mathrm{c} 428 \mathrm{r} \\
430+521+\mathrm{u}\end{array}$ & $\begin{array}{l}\text { BI: VIIb-VIII (Godwin 1975) NL: } 1400 \\
\text { B.C. (Pals et al. 1980) }\end{array}$ \\
\hline Polygonum cf. hydropiper L. & RIA: 301c GIA: 301r & \\
\hline
\end{tabular}


Table 2 continued

\begin{tabular}{|c|c|c|}
\hline Species & $\begin{array}{l}\text { Period, site number (cf. Table 1) and number } \\
\text { of macrofossils reported }\end{array}$ & $\begin{array}{l}\text { Reports on finds from neighbour countries. } \\
\text { Pollen zones and other periods are presented } \\
\text { as published by the authors cited }\end{array}$ \\
\hline $\begin{array}{l}\text { Polygonum lapathifolium L. } \\
\text { (P. tomentosum Schrank) }\end{array}$ & $\begin{array}{l}\text { VIII: } 127 \mathrm{c} 207 \mathrm{r} 219 \mathrm{r} 220 \mathrm{c} 230 \mathrm{r} 411 \mathrm{c} 543 \mathrm{r} 1065 \mathrm{r} \\
\text { PRIA: } 109+111 \mathrm{c} 114 \mathrm{c} 115 \mathrm{c} 121+141 \mathrm{c} 148 \mathrm{c} \\
201+301+\text { RIA: } 101 \mathrm{r} 107 \mathrm{c} 108 \mathrm{c} 131 \mathrm{r} 134 \mathrm{r} \\
136 \mathrm{r} 140+146+301 \mathrm{c} 302 \mathrm{r} 303 \mathrm{c} 306 \mathrm{r} 308 \mathrm{c} 309 \mathrm{c} \\
311 \mathrm{c} 315 \mathrm{r} 316 \mathrm{c} \text { GIA: } 130 \mathrm{r} 133 \mathrm{r} 135+222 \mathrm{r} \\
242+243 \mathrm{c} 244 \mathrm{c} 301+306 \mathrm{r} 713+718 \mathrm{r} \text { VA: } \\
103 \mathrm{c} 110+172 \mathrm{c} 202+301+304 \mathrm{c} 307 \mathrm{c} 312 \mathrm{c} \\
320 \mathrm{c} 33 \mathrm{c} 985+\text { EMA: } 172 \mathrm{c} 245 \mathrm{c} 428 \mathrm{c} 429 \mathrm{c} \\
431 \mathrm{c} 521 \mathrm{cu} 992+\text { LMA: } 217 \mathrm{cu} 218 \mathrm{c} 241 \mathrm{c} 245 \mathrm{c} \\
406 \mathrm{c} 427+428+429+521+\mathrm{u} 522+809+\end{array}$ & $\begin{array}{l}\text { BI: VIIb-VIII (Godwin 1975) NL: } 3400- \\
3300 \text { B.C. (Zeist \& Palfenier-Vegter 1981) } \\
\text { P: Neolithic (Gluza \& Wasylikowa 1977) }\end{array}$ \\
\hline $\begin{array}{l}\text { Polygonum lapathifolium } \mathrm{L} \text {. } \\
\text { et P. persicaria } \mathrm{L} \text {. }\end{array}$ & GIA: 205c EMA: 245c LMA: 245c & \\
\hline Polygonum cf. minus Hudson & EMA: $428 \mathrm{r}$ & $\begin{array}{l}\text { Polygonum minus recorded from BI: I/II, } \\
\text { VIIb-VIII (Godwin 1975) NL: } 2005 \pm 50 \\
\text { B.C. (Zeist 1968) BRD: Hallstatt (Knörzer } \\
\text { 1971c) P: Migration Period and later (Gluza } \\
\text { \& Wasylikowa 1977) N: 400-550 A.D. } \\
\text { (Griffin 1981) }\end{array}$ \\
\hline Polygonum cf. mite Schrank & RIA: $301+316$ r GIA: 301r VA: 301r & $\begin{array}{l}\text { Polygonum mite recorded from BI: VII-VIII } \\
\text { (Godwin 1975) BRD: 1st century A.D. } \\
\text { (Knörzer 1967a, 1970) }\end{array}$ \\
\hline Polygonum persicaria $\mathrm{L}$. & $\begin{array}{l}\text { VIII: } 116+301 \mathrm{c} 425+543 \text { PRIA: } 111+115 \mathrm{c} \\
\text { 301+ RIA: } 107+108 \mathrm{r} 139+301 \mathrm{c} 306 \mathrm{r} 311 \mathrm{c} \\
\text { GIA: } 135 \mathrm{r} 242 \mathrm{r} 243+244+301+\text { VA: } 172 \mathrm{c} \\
\text { 202r 301+304c 307c 312c 320c 334c } 985 \mathrm{c} \\
\text { EMA: } 172 \mathrm{c} 427+428+429 \mathrm{c} 431 \mathrm{c} 521 \mathrm{cu} 992 \mathrm{r} \\
\text { LMA: } 218 \mathrm{r} 241 \mathrm{c} 406 \mathrm{r} 427+428+429+430+ \\
\text { 521ru 522r } 809 \mathrm{r}\end{array}$ & $\begin{array}{l}\text { BI: I, III-V, VIIb-VIII (Godwin 1975) NL: } \\
\text { 3400-3300 B.C. (Zeist \& Palfenier-Vegter } \\
\text { 1981) BRD: Band Ceramic (Knörzer 1974a, } \\
\text { 1977) P: Neolithic, Bronze Age (Gluza \& } \\
\text { Wasylikowa 1977) }\end{array}$ \\
\hline Polygonum cf. persicaria L. & RIA: $308+309+$ & \\
\hline Polygonum sp. & $\begin{array}{l}\text { VIII: } 144 \mathrm{r} \text { 525r PRIA: 301r RIA: } 115 \mathrm{c} 125 \mathrm{c} \\
\text { 139c 151c 708c GIA: } 228+242+243+244+ \\
\text { 301r 712c VA: 172c 301r EMA: } 172+245 \mathrm{c} \\
428 \mathrm{c} 429 \mathrm{c} 431 \mathrm{c} \text { LMA: } 241+245+427+428+ \\
429+430+\end{array}$ & \\
\hline Populus tremula $\mathrm{L}$. & $\begin{array}{l}\text { IV: } 209 \mathrm{c} 238 \mathrm{ru} 508 \mathrm{cu} \text { V: } 238 \mathrm{r} 508 \mathrm{cu} 539 \mathrm{ru} \text { VI, } \\
\text { VII: } 501+508 \mathrm{c} 512+\mathrm{u} 422 \mathrm{ru} 501+508 \mathrm{cu} \\
\text { VIII: } 501+\text { VA: } 103 \mathrm{r}\end{array}$ & \\
\hline $\begin{array}{l}\text { Potamogeton alpinus Balbis } \\
\text { (P. alpinum) }\end{array}$ & II: 104 r 155r 510c 562r III: 417ru V: $104 r$ & \\
\hline $\begin{array}{l}\text { Potamogeton compressus L. } \\
\text { (P. zosterifolius Schumacher) }\end{array}$ & I: 510r II: 417ru III: 152 ru & \\
\hline Potamogeton filiformis Pers. & $\begin{array}{l}\text { I: } 113 \text { r 155r 510r II: } 104+113+155+510 \mathrm{r} \\
\text { III: } 104 \mathrm{r} 138 \mathrm{ru} 161 \mathrm{cu} 415 \mathrm{ru} 540 \mathrm{r} 995 \mathrm{r} \text { V: } 603 \mathrm{ru}\end{array}$ & BI: I-IV, VIIa (Godwin 1975) \\
\hline $\begin{array}{l}\text { Potamogeton cf. filiformis } \\
\text { Pers. }\end{array}$ & I: 511r II: 511c III: $506 \mathrm{cu} 516+\mathrm{u}$ & \\
\hline Potamogeton friesii Rupr. & III: $152 \mathrm{ru} 153 \mathrm{ru}$ & BI: II-IV (Godwin 1975) \\
\hline Potamogeton gramineus L. & III: 152 ru 415 ru & BI: I-III, VI-VIII (Godwin 1975) \\
\hline Potamogeton natans $L$. & $\begin{array}{l}\text { II: } 538+\text { III: 152ru 161ru 238+ 415ru 540r } \\
\text { 704ru 905r 995r IV: 209r 711c 905+ V: 104r } \\
\text { 410r 905+ 952r VI, VII: 410r 512cu 905r 164r } \\
\text { 410r 422cu 711r 905+906+ 944r VIII: 410r } \\
\text { 711r 906r 1036r IX: 209cu 711c } 905+906+ \\
\text { RIA: 311r }\end{array}$ & BI: I-VIII (Godwin 1975) \\
\hline Potamogeton cf. natans $L$. & II: 418r V: 555r VI, VII: 549r & \\
\hline
\end{tabular}


Table 2 continued

\begin{tabular}{|c|c|c|}
\hline Species & $\begin{array}{l}\text { Period, site number (cf. Table 1) and number } \\
\text { of macrofossils reported }\end{array}$ & $\begin{array}{l}\text { Reports on finds from neighbour countries. } \\
\text { Pollen zones and other periods are presented } \\
\text { as published by the authors cited }\end{array}$ \\
\hline $\begin{array}{l}\text { Potamogeton obtusifolius } \\
\text { Mert. \& Koch in Röhling }\end{array}$ & $\begin{array}{l}\text { III: 417ru 503ru VI, VII: 944r VIII: 1036r IX: } \\
\text { 1036r }\end{array}$ & BI: II-VIIa, VIII (Godwin 1975) \\
\hline Potamogeton pectinatus $\mathrm{L}$. & $\begin{array}{l}\text { III: 704ru 995r IV: 995r V: 213r 221r VI, VII: } \\
\text { 213r 221r VIII: 322r 323r 543r 822c }\end{array}$ & \\
\hline $\begin{array}{l}\text { Potamogeton cf. pectinatus } \\
\text { L. }\end{array}$ & $\begin{array}{l}\text { VI, VII: 321r } 326+\text { VIII: } 326+\text { GIA: } 301 r \\
\text { VA: } 301 \text { r }\end{array}$ & \\
\hline Potamogeton perfoliatus $\mathbf{L}$. & $\begin{array}{l}\text { I: } 113 \mathrm{r} \text { II: } 113+\text { IV: } 711 \mathrm{c} \text { V: } 555+u \text { VI, VII: } \\
\text { 520cu VA: } 304 \mathrm{r} 334+\end{array}$ & BI: I-III, VI-VIII (Godwin 1975) \\
\hline $\begin{array}{l}\text { Potamogeton praelongus } \\
\text { Wulfen }\end{array}$ & $\begin{array}{l}\text { I: } 113+416 \mathrm{cu} 417 \mathrm{ru} 510 \mathrm{r} 511 \mathrm{r} \text { II: } 113+416 \mathrm{cu} \\
\text { 417ru 419c 510c 511c 546cu 562+ 714r III: } \\
\text { 158+u 161+u 416ru 417ru 426ru 506+u IV: } \\
\text { 138ru 157r 711r V: } 157 \mathrm{r} 168 \mathrm{r} 506+\text { VI, VII: } \\
\text { 157c 168r 410r 501ru 157c 168r 410r 422ru } \\
\text { 520ru VIII: 157c 168r 410r IX: } 156 \mathrm{r} 168 \mathrm{r} 513 \mathrm{r}\end{array}$ & BI: I-VIII (Godwin 1975) \\
\hline $\begin{array}{l}\text { Potamogeton cf. praelongus } \\
\text { Wulfen }\end{array}$ & III: 415ru & \\
\hline Potamogeton pusillus $\mathrm{L}$. & III: $161+\mathrm{u} 415 \mathrm{ru} 503 \mathrm{ru}$ & \\
\hline $\begin{array}{l}\text { Potamogeton trichoides } \\
\text { Cham. \& Schlecht. }\end{array}$ & III: 152 ru & BI: II, VIII (Godwin 1975) \\
\hline $\begin{array}{l}\text { Potamogeton vaginatus } \\
\text { Turcz. }\end{array}$ & II: $155 \mathrm{r}$ & \\
\hline $\begin{array}{l}\text { Potamogeton } \mathrm{x} \text { zizii Koch ex } \\
\text { Roth }\end{array}$ & II: 416ru, 417ru III: 417ru VA: 307r & \\
\hline Potamogeton sp. & 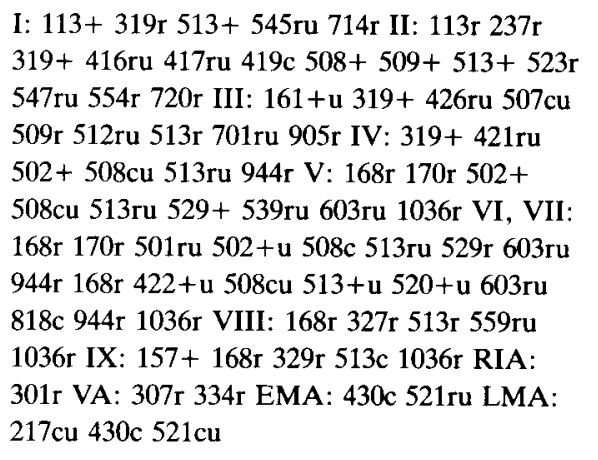 & \\
\hline $\begin{array}{l}\text { Potentilla anserina L. } \\
\text { (Argentina anserina (L.) } \\
\text { Rydberg) }\end{array}$ & $\begin{array}{l}\text { V: } 104 \text { r IX: 209r RIA: } 101+139 \text { r 301+306+ } \\
\text { 311c GIA: 301r VA: } 301+304+307 \mathrm{c} 334 \mathrm{c} \\
\text { EMA: } 245+430+431+521 \mathrm{cu} 522+\text { LMA: } \\
245+521 \mathrm{cu}\end{array}$ & BI: I-IV, VIIb-VIII (Godwin 1975) \\
\hline Potentilla argentea L. & $\begin{array}{l}\text { PRIA: } 115 r \text { VA: } 202 \text { r EMA: } 172+245+431 c \\
\text { LMA: } 241+\end{array}$ & $\begin{array}{l}\text { P: Hallstatt and later (Gluza \& Wasylikowa } \\
\text { 1977) }\end{array}$ \\
\hline Potentilla cf. argentea L. & EMA: $429 \mathrm{c}$ & \\
\hline $\begin{array}{l}\text { Potentilla erecta (L.) } \\
\text { Räuchel (P. tormentilla } \\
\text { Stokes) }\end{array}$ & $\begin{array}{l}\text { III: 161ru VIII: 207c 219c 229r 230r IX: 328+ } \\
\text { 501r PRIA: 115r 301r RIA: 101c 139r 301c } \\
\text { GIA: } 135+243+244+301 \text { r VA: 103+ 202r } \\
\text { 301r 304c 307+ 334c 564r EMA: 564+ LMA: } \\
\text { 241c 564c }\end{array}$ & BI: II-III, VI-VIII (Godwin 1975) \\
\hline $\begin{array}{l}\text { Potentilla palustris (L.) Scop. } \\
\text { (Comarum palustre L.) }\end{array}$ & $\begin{array}{l}\text { I: } 113 \text { r } 155 \text { c II: } 155+237 \text { r III: } 155+158+u \\
\text { 161ru } 165 \text { r 330r } 506+\text { u IV: } 165 \text { r } 209 \text { r } 235 \mathrm{cu} \\
\text { 330c } 502+\text { V: } 502+603 \text { ru } 1090+\text { VI, VII: } \\
501+\text { u 502+u } 502+508+\text { u } 944 \text { r VIII: } 328 r \\
502+513 \text { ru } 559 \text { ru IX: } 327 \text { r } 328 \text { r } 329 \text { r } 332+\end{array}$ & BI: I-VIII (Godwin 1975) \\
\hline
\end{tabular}


Table 2 continued

\begin{tabular}{|c|c|c|}
\hline Species & $\begin{array}{l}\text { Period, site number (cf. Table 1) and number } \\
\text { of macrofossils reported }\end{array}$ & $\begin{array}{l}\text { Reports on finds from neighbour countries. } \\
\text { Pollen zones and other periods are presented } \\
\text { as published by the authors cited }\end{array}$ \\
\hline $\begin{array}{l}\text { Potentilla palustris (L.) Scop. } \\
\text { (continued) }\end{array}$ & $\begin{array}{l}\text { 502+ 513+ 711r RIA: 101c GIA: } 244+\text { VA: } \\
\text { 202r 334c EMA: } 172 \text { r 431+ 521ru LMA: } \\
\text { 217cu 218c 522r }\end{array}$ & \\
\hline $\begin{array}{l}\text { cf. Potentilla palustris (L.) } \\
\text { Scop. }\end{array}$ & EMA: $429+$ & \\
\hline Potentilla reptans $\mathrm{L}$. & GIA: $242+$ LMA: $428 \mathrm{r}$ & $\begin{array}{l}\text { BI: VIIa, Roman Period (Godwin 1975), } \\
3360 \pm 80 \text { B.P. (Peglar \& Wilson 1978), 100- } \\
300 \text { A.D. (Wilson 1968) NL: 1st-3rd century } \\
\text { A.D. (Zeist 1974) BRD: 1st century A.D. } \\
\text { (Knörzer 1970) }\end{array}$ \\
\hline Potentilla cf. reptans L. & VA: $202 r$ & \\
\hline Potentilla sp. & $\begin{array}{l}\text { II: } 104 \mathrm{r} \text { III: 161ru 507ru VIII: } 116+609+ \\
\text { PRIA: 301r RIA: } 301+\text { GIA: } 242+244+301 \mathrm{r} \\
\text { VA: } 172+202 \mathrm{r} 301 \mathrm{r} \text { EMA: } 172+245+428 \mathrm{r} \\
\text { 429c } 430+431 \mathrm{c} 521 \mathrm{cu} \text { LMA: } 241+427 \mathrm{c} 428 \mathrm{r} \\
521+\mathrm{u}\end{array}$ & \\
\hline Primula sp. & LMA: 521ru & $\begin{array}{l}\text { BI: Roman Period (Godwin 1975) P: 11th- } \\
\text { 12th century A.D. (Wasylikowa 1978). } \\
\text { Primula veris recorded from P: Hallstatt and } \\
\text { later (Gluza \& Wasylikowa 1977), from S: } \\
\text { 1300 A.D. (Griffin 1982), and N: 1150-1225 } \\
\text { A.D. (Griffin 1979a) }\end{array}$ \\
\hline Prunella vulgaris $\mathrm{L}$. & $\begin{array}{l}\text { V: 104r VIII: 220r 543r PRIA: } 115+\text { RIA: } \\
\text { 101+ 301r GIA: 301r 712r VA: 301r 304c 307r } \\
\text { 334c EMA: } 172+428 \mathrm{c} 429 \mathrm{c} 431+521 \mathrm{cu} \\
\text { LMA: } 218+241 \mathrm{c} 406 \mathrm{r} 427 \mathrm{c} 521 \mathrm{cu} 522+\end{array}$ & BI: V, VIIb-VIII (Godwin 1975) \\
\hline $\begin{array}{l}\text { Prunus avium L. (Cerasus } \\
\text { avium (L.) Moench) }\end{array}$ & VA: 304 r $312+334+$ LMA: 522 r & $\begin{array}{l}\text { BI: VIIb-VIII (Godwin 1975) NL: Roman } \\
\text { Period (Zeist 1968) BRD: 1st century A.D. } \\
\text { and later (Knörzer 1967b, 1970, 1973), 6th- } \\
\text { 7th century A.D. (Bertsch 1927) DDR: 7th- } \\
\text { 8th century A.D. (Jäger 1966) P: Hallstatt } \\
\text { and later (Gluza \& Wasylikowa 1977) }\end{array}$ \\
\hline cf. Prunus avium L. & LMA: $428 \mathrm{r}$ & \\
\hline Prunus cerasus L. & LMA: $406+985 r$ & $\begin{array}{l}\text { BI: 13th century A.D. (Godwin 1975) BRD: } \\
\text { 13th-14th century A.D. (Westhusen 1958, } \\
\text { Kroll 1978), Late Middle Ages (Willerding } \\
\text { 1978) P: Early and Late Medieval (Gluza \& } \\
\text { Wasylikowa 1977) }\end{array}$ \\
\hline $\begin{array}{l}\text { Prunus subgen. Cerasus } \\
\text { (Miller) Focke (Cerasus sp.) }\end{array}$ & EMA: $521+$ u LMA: 521 ru & \\
\hline Prunus domestica L. & EMA: $305+$ 521ru LMA: 218 r $305+$ & $\begin{array}{l}\text { BI: VIIb-VIII (Godwin 1975), c. } 300 \text { A.D. } \\
\text { (Wilson 1978), Roman Period (Willcox 1977) } \\
\text { BRD: Neolithic, Roman Period (Knörzer } \\
\text { 1973), 1st century A.D. (Knörzer 1967b, } \\
\text { 1970), c. } 200 \text { A.D. (Körber-Grohne 1979b) } \\
\text { P: Early Medieval (Gluza \& Wasylikowa } \\
\text { 1977), first half 11th century A.D. (Kosina } \\
\text { 1978) N: Middle Ages (Krzywinski 1979, } \\
\text { Griffin 1981) }\end{array}$ \\
\hline $\begin{array}{l}\text { Prunus domestica L. subsp. } \\
\text { insititia (L.) C. K. Schneider } \\
\text { (P. insititia L.) } \\
\text { (to be continued) }\end{array}$ & $\begin{array}{l}\text { VA: } 304+310 c 312 c 334 c 408+985+\text { EMA: } \\
\text { 305c LMA: } 305 c 406+\end{array}$ & $\begin{array}{l}\text { BI: } 3360 \pm 80 \text { B.P. (Peglar \& Wilson 1978), } \\
\text { Iron Age, Roman and Anglo-Saxon Period } \\
\text { (Godwin 1975) NL: 5th-9th century A.D. } \\
\text { (Zeist 1968) BRD: Band Ceramic (Knörzer } \\
\text { 1974a), 2nd century A.D. (Knörzer 1973), }\end{array}$ \\
\hline
\end{tabular}




\begin{tabular}{|c|c|c|}
\hline Species & $\begin{array}{l}\text { Period, site number (cf. Table 1) and number } \\
\text { of macrofossils reported }\end{array}$ & $\begin{array}{l}\text { Reports on finds from neighbour countries. } \\
\text { Pollen zones and other periods are presented } \\
\text { as published by the authors cited }\end{array}$ \\
\hline $\begin{array}{l}\text { Prunus domestica L. subsp. } \\
\text { insititia (L.) C. K. Schneider } \\
\text { (continued) }\end{array}$ & & $\begin{array}{l}\text { 10th-11th century A.D. (Wittmack \& } \\
\text { Buchwald 1902) P: Early Medieval (Gluza \& } \\
\text { Wasylikowa 1977) }\end{array}$ \\
\hline $\begin{array}{l}\text { Prunus cf. domestica L. } \\
\text { subsp. insititia (L.) C. K. } \\
\text { Schneider }\end{array}$ & EMA: $430+$ & \\
\hline Prunus padus L. & IV: 209r VI, VII: 422ru & \\
\hline Prunus persica (L.) Batsch & VA: 312 r $334 r$ & $\begin{array}{l}\text { BI: 1st-2nd century A.D. (Willcox 1977) } \\
\text { BRD: 1st century A.D. and later (Knörzer } \\
\text { 1967c, 1970, 1973), 6th-7th century A.D. } \\
\text { (Bertsch 1927) P: Early Middle Ages (Gluza } \\
\text { \& Wasylikowa 1977) }\end{array}$ \\
\hline Prunus spinosa $\mathbf{L}$. & $\begin{array}{l}\text { VIII: 409r VA: } 304+307 r \text { r 312r 334c } 985 r \\
\text { EMA: } 521+u \text { LMA: } 406 \mathrm{c} 985 \mathrm{c}\end{array}$ & $\begin{array}{l}\text { BI: VIIa-VIII (Godwin 1975) BRD: Band } \\
\text { Ceramic (Knörzer 1977) P: Neolithic (Gluza } \\
\text { \& Wasylikowa 1977) }\end{array}$ \\
\hline Puccinellia distans (L.) Parl. & RIA: 311c VA: 307c & $\begin{array}{l}\text { NL: } 600-400 \text { B.C. (Zeist 1974) BRD: 1st- } \\
\text { 2nd century (Körber-Grohne 1967) }\end{array}$ \\
\hline $\begin{array}{l}\text { Puccinellia maritima } \\
\text { (Hudson) Parl. }\end{array}$ & RIA: $311 \mathrm{c}$ VA: $307 \mathrm{c}$ & NL: $600-400$ B.C. (Zeist 1974) \\
\hline $\begin{array}{l}\text { Puccinellia cf. maritima } \\
\text { (Hudson) Parl. }\end{array}$ & GIA: $301 \mathrm{r}$ & \\
\hline Pyrus communis $\mathrm{L}$. & LMA: $406 \mathrm{c}$ & $\begin{array}{l}\text { P: 9th-12th century A.D. (Wasylikowa } \\
\text { 1978), Early Medieval Age (Gluza \& } \\
\text { Wasylikowa 1977) }\end{array}$ \\
\hline Pyrus sp. & EMA: $521+u$ LMA: $521+u$ & $\begin{array}{l}\text { BI: VIIb-VIII (Godwin 1975) BRD: c. } 1209 \\
\text { A.D. (Lynch \& Paap 1982). Pyrus communis } \\
\text { recorded from BRD: 1st century A.D. and } \\
\text { later (Knörzer 1973), and from P: 9th-12th } \\
\text { century A.D. (Wasylikowa 1978), Early } \\
\text { Medieval (Gluza \& Wasylikowa 1977) }\end{array}$ \\
\hline $\begin{array}{l}\text { Quercus petraea } \\
\text { (Mattuschka) Liebl. et } Q \text {. } \\
\text { robur L. }\end{array}$ & VIII: $206 \mathrm{c}$ & \\
\hline $\begin{array}{l}\text { Quercus cf. petraea } \\
\text { (Mattuschka) Liebl. (Q. } \\
\text { sessiliflora Salisb.) }\end{array}$ & VI, VII: $164 \mathrm{r}$ & $\begin{array}{l}\text { Quercus petraea recorded from BI: VI/VIIa- } \\
\text { VIIb (Godwin 1975) }\end{array}$ \\
\hline $\begin{array}{l}\text { Quercus robur L. (Q. } \\
\text { pedunculata Ehrh.) }\end{array}$ & $\begin{array}{l}\text { VI, VII: } 422 \mathrm{cu} 502+508 \mathrm{cu} 513 \mathrm{cu} \text { VIII: } 502+ \\
508+\text { u } 513 \mathrm{cu} \text { IX: } 502+513+\end{array}$ & \\
\hline Quercus cf. robur $\mathbf{L}$. & $\begin{array}{l}\text { VI, VII: } 528 \mathrm{ru} 501+\text { VIII: } 501+559+\mathrm{u} \mathrm{IX:} \\
501+\end{array}$ & \\
\hline Quercus sp. & $\begin{array}{l}\text { VI, VII: } 235+u \text { 410r 159r } 235+u \text { 410r VIII: } \\
\text { 410r 543r IX: 711c VA: } 304+307 \text { r 312+334+ } \\
\text { 985c LMA: } 217 \text { ru }\end{array}$ & \\
\hline $\begin{array}{l}\text { Ranunculus acris L. (R. acer } \\
\text { L.) }\end{array}$ & $\begin{array}{l}\text { VIII: 214r PRIA: 115r RIA: 101r 108c 306+ } \\
\text { 311r GIA: 306+ VA: 304c 307c 334c 985r } \\
\text { EMA: } 521+\text { u LMA: } 218 \mathrm{r} 241+521+\mathrm{u}\end{array}$ & $\begin{array}{l}\text { BI: II-IV, VI-VIII (Godwin 1975) NL: } \\
\text { 3400-3300 B.C. (Zeist \& Palfenier-Vegter } \\
\text { 1981) }\end{array}$ \\
\hline Ranunculus cf. acris L. & $\begin{array}{l}\text { PRIA: 301r RIA: 301r GIA: 301r VA: 301r } \\
\text { EMA: 428r }\end{array}$ & \\
\hline $\begin{array}{l}\text { Ranunculus aquatilis } \mathrm{L} \text {. } \\
\text { (Batrachium aquatile (coll.)) }\end{array}$ & $\begin{array}{l}\text { I: } 104 \mathrm{r} \text { II: } 104+\text { III: } 158+\mathrm{u} 161 \mathrm{cu} 423 \mathrm{ru} \text { V: } \\
\text { 104r }\end{array}$ & \\
\hline Ranunculus cf. aquatilis $L$. & II: $155+$ III: $155+161 \mathrm{cu}$ & \\
\hline
\end{tabular}


Table 2 continued

\begin{tabular}{|c|c|c|}
\hline Species & $\begin{array}{l}\text { Period, site number (cf. Table 1) and number } \\
\text { of macrofossils reported }\end{array}$ & $\begin{array}{l}\text { Reports on finds from neighbour countries. } \\
\text { Pollen zones and other periods are presented } \\
\text { as published by the authors cited }\end{array}$ \\
\hline $\begin{array}{l}\text { Ranunculus subgen. } \\
\text { Batrachium }\end{array}$ & $\begin{array}{l}\text { I: 319r II: 113r 319r 513r 523+ 538r 547ru } \\
\text { 562+ 720r III: 113+ 319r 507ru 719+u 905+ } \\
\text { IV: 138ru 209r V: 213r 221r 603ru VI, VII: } \\
\text { 213r 221r 501ru 549r RIA: 301r VA: 202r 307r } \\
\text { 334c EMA: 521+u LMA: } 217+\text { u 428+ 521cu } \\
\text { 522r }\end{array}$ & BI: I-VIII (Godwin 1975) \\
\hline Ranunculus cf. bulbosus L. & LMA: $427+$ & $\begin{array}{l}\text { Ranunculus bulbosus recorded from BI: } \\
\text { Late Weichselian, VIIb-VIII (Godwin 1975), } \\
3360 \pm 80 \text { B.P. (Peglar \& Wilson 1978), 2nd } \\
\text { century A.D. (Wilson 1979), and N: } 1150- \\
1250 \text { A.D. (Griffin 1979a) }\end{array}$ \\
\hline Ranunculus flammula $\mathbf{L}$. & $\begin{array}{l}\text { IV: 104r VI, VII: 410r 410r VIII: 410r IX: } \\
\text { 180c RIA: 101c 301c 306+ GIA: } 242+243+ \\
\text { 244c VA: 172r 202c 304+ 334c EMA: 172r } \\
\text { 428c 429c 431+ 521cu LMA: 241c 427c } 428+ \\
429+521+u\end{array}$ & BI: I-IV, VI-VIII (Godwin 1975) \\
\hline $\begin{array}{l}\text { Ranunculus cf. lanuginosus } \\
\text { L. }\end{array}$ & VA: $304+334+$ & \\
\hline Ranunculus lingua $\mathrm{L}$. & RIA: $311 \mathrm{c}$ VA: $307+$ & $\begin{array}{l}\text { BI: I-V, VIIb-VIII (Godwin 1975), c. } 300 \\
\text { A.D. (Wilson 1978), 4th-5th century A.D. } \\
\text { (Greig 1976) NL: 100-250 A.D. (Zeist 1974) }\end{array}$ \\
\hline Ranunculus cf. lingua $\mathrm{L}$. & VA: $301 \mathrm{r}$ & \\
\hline Ranunculus parviflorus $\mathrm{L}$. & EMA: $521+u$ & $\begin{array}{l}\text { BI: } 3360 \pm 80 \text { B.P. (Peglar \& Wilson 1978), } \\
\text { 3rd century B.C. (Wilson 1978) }\end{array}$ \\
\hline Ranunculus repens $\mathbf{L}$. & $\begin{array}{l}\text { IV: } 104 \mathrm{r} \text { V: } 104+\text { VI, VII: 549r VIII: 213ru } \\
\text { 220r 421ru 543r IX: 501r PRIA: 115+ RIA: } \\
101+301+306+311 \mathrm{c} \text { GIA: } 135 \mathrm{c} 301+553 \mathrm{r} \\
\text { VA: } 202+301 \mathrm{r} 304+307 \mathrm{c} 34 \mathrm{c} \text { EMA: } 427+ \\
428+429+431+521 \mathrm{cu} 522 \mathrm{r} \text { LMA: 171r } 217+ \\
218 \mathrm{c} 241 \mathrm{c} 406+427 \mathrm{c} 428+521 \mathrm{cu} 522+\end{array}$ & BI: I-VIII (Godwin 1975) \\
\hline Ranunculus cf. repens $L$. & VA: $172+$ EMA: $172+$ & \\
\hline Ranunculus sardous Crantz & $\begin{array}{l}\text { RIA: } 311+\text { VA: 172r EMA: } 429+\text { LMA: } \\
427+\end{array}$ & $\begin{array}{l}\text { BI: Roman Period (Godwin 1975), 4th-5th } \\
\text { century A.D. (Greig 1976) NL: Before } 1400 \\
\text { B.C. (Pals et al. 1980) BRD: 12-8 B.C. } \\
\text { (Kučan 1981), 1st century A.D. (Knörzer } \\
\text { 1967b, 1970) }\end{array}$ \\
\hline $\begin{array}{l}\text { Ranunculus sceleratus L. } \\
\text { (Batrachium sceleratum) }\end{array}$ & $\begin{array}{l}\text { VI, VII: 549c VIII: } 543+\text { PRIA: } 301+\text { RIA: } \\
\text { 301+ 311c GIA: } 242+243 \mathrm{c} 244 \mathrm{c} 301+\text { VA: } \\
\text { 172c 301c 304c 307c 334c 543r 985c EMA: } \\
172+245 \mathrm{c} 427+428 \mathrm{c} 429 \mathrm{c} 430 \mathrm{c} 431 \mathrm{c} 521 \mathrm{cu} \\
\text { LMA: } 241+245 \mathrm{c} 427 \mathrm{c} 428 \mathrm{c} 430 \mathrm{c} 521 \mathrm{cu}\end{array}$ & $\begin{array}{l}\text { BI: II-IV, VI, VIIb-VIII (Godwin 1975) } \\
\text { NL: 3400-3300 B.C. (Zeist \& Palfenier- } \\
\text { Vegter 1981) BRD: 5500-4000 B.C. (Stalling } \\
\text { 1983) }\end{array}$ \\
\hline $\begin{array}{l}\text { Ranunculus cf. trichophyllus } \\
\text { Chaix in Vill. (Batrachium } \\
\text { confervoides Fries) }\end{array}$ & $\begin{array}{l}\text { I: } 510+511+545 \text { ru II: } 510 \mathrm{c} 511 \mathrm{c} 546 \mathrm{ru} 554 \mathrm{r} \\
\text { III: } 415 \mathrm{ru} 510 \mathrm{r} 511 \mathrm{r} 512 \mathrm{r} \text { IV: } 238 \mathrm{ru}\end{array}$ & \\
\hline Ranunculus sp. & $\begin{array}{l}\text { III: 161ru RIA: 301r GIA: } 242+244+301+ \\
\text { VA: 172r 202+ 301r 304r EMA: } 245+428+ \\
\text { 429+ LMA: } 245 \text { c } 427+430+\end{array}$ & \\
\hline Raphanus raphanistrum L. & $\begin{array}{l}\text { VIII: 208r 213ru 214r 221r RIA: 301c GIA: } \\
205+243+244+301 \text { r VA: } 103+110 \text { r 172+ } \\
301+304 \text { c 307+334c EMA: } 172 \text { r } 245+428+ \\
429+431+521 \mathrm{cu} 522 \text { r 709r LMA: } 218 \mathrm{c} 241+ \\
406 \text { r } 427+428+429+430+521+u \text { u } 522+\end{array}$ & BI: VIIb-VIII (Godwin 1975) \\
\hline Rhinanthus cf. minor $L$. & PRIA: $115 r$ & $\begin{array}{l}\text { Rhinanthus minor recorded from P: Hallstatt } \\
\text { and later (Gluza \& Wasylikowa 1977) }\end{array}$ \\
\hline
\end{tabular}


Table 2 continued

\begin{tabular}{|c|c|c|}
\hline Species & $\begin{array}{l}\text { Period, site number (cf. Table 1) and number } \\
\text { of macrofossils reported }\end{array}$ & $\begin{array}{l}\text { Reports on finds from neighbour countries. } \\
\text { Pollen zones and other periods are presented } \\
\text { as published by the authors cited }\end{array}$ \\
\hline Rhinanthus sp. & RIA: $311+$ VA: 202 r $307+334 c$ & \\
\hline Rhynchospora alba (L.) Vahl & VIII: $331 \mathrm{r}$ IX: $331+332 \mathrm{r}$ & BI: V, VIIb-VIII (Godwin 1975) \\
\hline Rhynchospora sp. & VI, VII: $164 \mathrm{r}$ & $\begin{array}{l}\text { Rhynchospora cf. alba recorded from BI: I, } \\
\text { R. alba from V, VIIb-VIII (Godwin 1975) }\end{array}$ \\
\hline $\begin{array}{l}\text { Rorippa islandica (Oeder) } \\
\text { Borbás (R. palustris (L.) } \\
\text { Besser) }\end{array}$ & RIA: $301 \mathrm{c}$ GIA: $242 \mathrm{r} 244+$ VA: $172+334 \mathrm{r}$ & $\begin{array}{l}\text { BI: Late Weichselian, IV, VIII (Godwin } \\
\text { 1975) NL: Before } 1400 \text { B.C. (Pals et al. } \\
\text { 1980) BRD: } 7200-4000 \text { B.C. (Stalling 1983), } \\
\text { 1st century A.D. (Knörzer 1967b, 1970), c. } \\
200 \text { A.D. (Körber-Grohne 1979b) }\end{array}$ \\
\hline Rorippa sp. & EMA: 521ru LMA: 521ru & \\
\hline Rosa sp. & $\begin{array}{l}\text { VA: } 334+\text { EMA: } 430+521+u \text { LMA: } 430 \mathrm{c} \\
427+521+\mathrm{u} 522 \mathrm{r}\end{array}$ & $\begin{array}{l}\text { BI: VIIb-VIII (Godwin 1975), 100-300 } \\
\text { A.D. (Wilson 1968, 1978). Rosa canina L. } \\
\text { recorded from Late Neolithic and Roman } \\
\text { sites (Godwin 1975) NL: 3400-3300 B.C. } \\
\text { (Zeist \& Palfenier-Vegter 1981) BRD: Late } \\
\text { Neolithic (Knörzer 1967d), 12-8 B.C. } \\
\text { (Kučan 1981), 2nd century A.D. (Knörzer } \\
\text { 1973), c. 200 A.D. (Körber-Grohne 1979b). } \\
\text { Rosa canina recorded from P: Early } \\
\text { Medieval (Gluza \& Wasylikowa } \\
\text { 1977) }\end{array}$ \\
\hline Rubus caesius $\mathbf{L}$. & LMA: $406+428+$ & $\begin{array}{l}\text { BI: Iron Age (Godwin 1975) NL: } 2040 \pm 65 \\
\text { B.C. (Zeist 1968) BRD: Band Ceramic } \\
\text { (Knörzer 1974a), Neolithic and later } \\
\text { (Knörzer 1973), 1st century A.D. (Knörzer } \\
\text { 1967a), 10th century A.D. (Willerding } \\
\text { 1973), 1000-1200 A.D. (Willerding 1979b), } \\
\text { Early and Late Middle Ages (Willerding } \\
\text { 1978b) P: 9th-12th century A.D. } \\
\text { (Wasylikowa 1978), 11th century A.D. } \\
\text { (Kosina 1978), Early Medieval Age (Gluza } \\
\text { \& Wasylikowa 1977) N: Medieval Age } \\
\text { (Griffin 1981) }\end{array}$ \\
\hline Rubus cf. caesius $\mathrm{L}$. & VIII: $327 \mathrm{r}$ & \\
\hline Rubus corylifolius Sm. & EMA: $431+$ & \\
\hline Rubus fruticosus L. - group & $\begin{array}{l}\text { VIII: } 609 \text { c RIA: } 301 \text { r GIA: } 564+u \text { VA: } 172 \text { r } \\
\text { 202r 301r 304c 312c 334c 564r EMA: } 428+ \\
430 \mathrm{c} 431+521 \mathrm{cu} 522 \mathrm{r} 564+\text { LMA: } 406 \mathrm{c} 428 \mathrm{r} \\
429+430+521+\mathrm{u} 522+564 \mathrm{r}\end{array}$ & $\begin{array}{l}\text { BI: VIIa-VIII (Godwin 1975) NL: } 3400- \\
3300 \text { B.C. (Zeist \& Palfenier-Vegter 1981) }\end{array}$ \\
\hline Rubus cf. fruticosus $\mathbf{L}$. & GIA: $242 r$ & \\
\hline Rubus idaeus L. & $\begin{array}{l}\text { III: 161ru V: 235ru VI, VII: 410r 528ru 549c } \\
\text { 180r 410r 502+ 520+u 944r VIII: 208c 213ru } \\
\text { 221cu 329r 410r 502+ 531c 609+ IX: } 180 \mathrm{r} \\
\text { 502+ 513r RIA: 128r VA: } 172+202 \mathrm{r} 304 \mathrm{c} \\
\text { 312c 334c 564+ EMA: } 245 \mathrm{c} 428+521 \mathrm{ru} 564 \mathrm{c} \\
\text { LMA: } 217 \mathrm{r} 245+406+521+\mathrm{u} 564+\end{array}$ & \\
\hline Rubus saxatilis L. & II: $509+510 \mathrm{c} 511 \mathrm{c} \mathrm{V}: 539 \mathrm{ru}$ & BI: II, VI (Godwin 1975) \\
\hline Rubus sp. & GIA: $244+$ EMA: 172 r $429+$ LMA: $241+$ & \\
\hline Rumex acetosa L. & VIII: $220 \mathrm{r}$ VA: $304+307 \mathrm{r}$ & $\begin{array}{l}\text { BI: I-III, VIIb-VIII (Godwin 1975) NL: } 800 \\
\text { B.C. (Pals et al. 1980) P: Neolithic, Bronze } \\
\text { Age (Gluza \& Wasylikowa 1977) }\end{array}$ \\
\hline Rumex cf. acetosa L. & $\begin{array}{l}\text { PRIA: 114r RIA: } 301+\text { GIA: 242r VA: } 985 r \\
\text { LMA: 809r }\end{array}$ & \\
\hline
\end{tabular}


Table 2 continued

\begin{tabular}{|c|c|c|}
\hline Species & $\begin{array}{l}\text { Period, site number (cf. Table 1) and number } \\
\text { of macrofossils reported }\end{array}$ & $\begin{array}{l}\text { Reports on finds from neighbour countries. } \\
\text { Pollen zones and other periods are presented } \\
\text { as published by the authors cited }\end{array}$ \\
\hline Rumex acetosella L. & $\begin{array}{l}\text { II: } 155 \text { r III: } 155+\text { VIII: } 127+207 \mathrm{c} 219 \mathrm{r} 220 \mathrm{c} \\
230+425 \mathrm{r} \text { IX: } 180 \mathrm{r} \text { PRIA: } 109 \mathrm{c} 111 \mathrm{r} 114 \mathrm{r} \\
\text { 115+ 301+ RIA: } 101 \mathrm{c} 108 \mathrm{c} 139+301+308+ \\
\text { 316r GIA: } 135 \mathrm{r} 242 \mathrm{c} 243 \mathrm{c} 244 \mathrm{c} 301+\text { VA: } \\
\text { 103+ 172c 301+ 304c 307c 334c EMA: } 172 \mathrm{c} \\
245 \mathrm{c} 427+428 \mathrm{c} 429 \mathrm{c} 430+431 \mathrm{c} 521 \mathrm{cu} \text { LMA: } \\
218+241 \mathrm{c} 245 \mathrm{c} 406+427 \mathrm{c} 428 \mathrm{c} 429+430+ \\
521 \mathrm{cu} 522+564 \mathrm{r}\end{array}$ & BI: I-IV, VIIa-VIII (Godwin 1975) \\
\hline $\begin{array}{l}\text { Rumex conglomeratus } \\
\text { Murray }\end{array}$ & LMA: 406r & $\begin{array}{l}\text { BI: VIIa-VIII (Godwin 1975), } 2130 \pm 100 \\
\text { B.P. (Greig 1979b) NL: 3400-3300 B.C. } \\
\text { (Zeist \& Palfenier-Vegter 1981) BRD: 1st } \\
\text { century A.D. (Knörzer 1970), c. } 200 \text { A.D. } \\
\text { (Körber-Grohne 1979b), Late Middle Ages } \\
\text { (Knörzer 1975, Willerding 1978b) DDR: } \\
\text { 7th-9th century A.D. (Lange } \\
\text { 1976) }\end{array}$ \\
\hline $\begin{array}{l}\text { Rumex conglomeratus } \\
\text { Murray et R. sanguineus L. }\end{array}$ & VIII: $609 \mathrm{r}$ & \\
\hline Rumex crispus $\mathrm{L}$. & $\begin{array}{l}\text { VI, VII: 520ru PRIA: } 109+115 \text { r RIA: } 306 r \\
\text { 311r GIA: 242r 244+ 553r VA: } 110 \mathrm{c} 172 \mathrm{c} 304 \mathrm{c} \\
\text { 307c 334c 985r EMA: } 172+427 \mathrm{c} 428+429 \mathrm{c} \\
\text { 431c 521ru 992r LMA: } 218+427 \mathrm{c} 428+430+ \\
\text { 522r 809r }\end{array}$ & $\begin{array}{l}\text { BI: Late Weichselian, VIIb-VIII (Godwin } \\
\text { 1975) }\end{array}$ \\
\hline Rumex cf. crispus L. & $\begin{array}{l}\text { PRIA: 301r RIA: } 301+308+\text { GIA: 301r 712+ } \\
\text { VA: 301r 985r }\end{array}$ & \\
\hline $\begin{array}{l}\text { Rumex crispus } \mathbf{L} \text {. et } \mathbf{R} \text {. } \\
\text { obtusifolius } \mathbf{L} \text {. }\end{array}$ & RIA: $108 \mathrm{r}$ & \\
\hline $\begin{array}{l}\text { Rumex hydrolapathum } \\
\text { Hudson }\end{array}$ & $\begin{array}{l}\text { V: } 539 \mathrm{ru} \text { VI, VII: } 422 \mathrm{cu} \text { RIA: } 301+311 \mathrm{r} \\
\text { GIA: 553r VA: } 304 \mathrm{c} 307 \mathrm{c} 334 \mathrm{c} \text { LMA: } \\
217+\mathrm{u}\end{array}$ & \\
\hline $\begin{array}{l}\text { Rumex cf. hydrolapathum } \\
\text { Hudson }\end{array}$ & VI, VII: $549 \mathrm{r}$ & \\
\hline $\begin{array}{l}\text { Rumex longifolius DC. in } \\
\text { Lam. \& DC. (R. domesticus } \\
\text { Hartm.) }\end{array}$ & VA: $202 r$ & BI: Late Bronze Age (Godwin 1975) \\
\hline $\begin{array}{l}\text { Rumex cf. longifolius DC. in } \\
\text { Lam. \& DC. }\end{array}$ & GIA: 205r VA: 985r LMA: $241+$ & \\
\hline Rumex maritimus L. & $\begin{array}{l}\text { VI, VII: 520ru RIA: } 311+\text { GIA: } 306+\text { VA: } \\
\text { 110c EMA: 521ru LMA: 521cu }\end{array}$ & $\begin{array}{l}\text { BI: III-IV, VIII (Godwin 1975) NL: } 3400- \\
\text { 3300 B.C. (Zeist \& Palfenier-Vegter 1981) }\end{array}$ \\
\hline Rumex cf. maritimus L. & IV: 238ru V: 238ru & \\
\hline $\begin{array}{l}\text { Rumex maritimus L. et } \mathrm{R} \text {. } \\
\text { palustris } \mathrm{Sm} \text {. }\end{array}$ & LMA: $427+$ & \\
\hline Rumex obtusifolius L. & VA: 103+ EMA: 992r LMA: 406r 430+ 985r & $\begin{array}{l}\text { BI: II, VIII (Godwin 1975) NL: } 370 \pm 70 \\
\text { B.C. (Zeist 1974) P: Early Medieval (Gluza } \\
\text { \& Wasylikowa 1977), 9th-12th century A.D. } \\
\text { (Wasylikowa 1978) }\end{array}$ \\
\hline Rumex cf. obtusifolius L. & GIA: $244 c$ & \\
\hline $\begin{array}{l}\text { Rumex tenuifolius (Wallr.) } \\
\text { A. Löve }\end{array}$ & EMA: $992+$ & $\begin{array}{l}\text { BI: I-III, VIII (Godwin 1975) BRD: } \\
\text { Hallstatt (Knörzer 1971c, 1974b), c. } 200 \\
\text { B.C. (Knörzer 1979a), 1st century A.D. } \\
\text { (Knörzer 1967a, 1970), 2nd century A.D. } \\
\text { (Knörzer 1979b) P: 9th-12th century A.D. } \\
\text { (Wasylikowa 1978) }\end{array}$ \\
\hline
\end{tabular}




\begin{tabular}{|c|c|c|}
\hline Species & $\begin{array}{l}\text { Period, site number (cf. Table 1) and number } \\
\text { of macrofossils reported }\end{array}$ & $\begin{array}{l}\text { Reports on finds from neighbour countries. } \\
\text { Pollen zones and other periods are presented } \\
\text { as published by the authors cited }\end{array}$ \\
\hline Rumex sp. & $\begin{array}{l}\text { VI, VII: 322r 555r 323r 508ru IX: 332r PRIA: } \\
\text { 301+ RIA: } 301+303 \mathrm{c} 306 \text { r 311c } 316+\text { GIA: } \\
\text { 242r } 243+\text { VA: } 172+301 \text { r 304c 307c 334c } \\
985+\text { EMA: } 172+245+427+428+429 \mathrm{c} \\
431+521 \mathrm{cu} \text { LMA: } 427+428 \mathrm{r} 521 \mathrm{cu}\end{array}$ & \\
\hline $\begin{array}{l}\text { Ruppia cirrhosa (Petagna) } \\
\text { Grande (R. spiralis L. ex } \\
\text { Dumort.) }\end{array}$ & VI, VII: $520 \mathrm{cu} 553 \mathrm{cu}$ & BI: IV/V-VIII (Godwin 1975) \\
\hline Ruppia maritima L. & $\begin{array}{l}\text { V: } 104 r \text { VIII: } 213 c 221 c 405 r \text { 543r VA: } \\
307+\end{array}$ & BI: IV-V, VIIa-VIIb (Godwin 1975) \\
\hline Ruppia sp. & $\begin{array}{l}\text { VI, VII: 556c 558c } 818+826+\text { VIII: 556c 557r } \\
\text { 558r 818+826+ IX: 556c 557c 558r EMA: } \\
\text { 521cu LMA: 521ru }\end{array}$ & \\
\hline Sagina cf. procumbens L. & RIA: $301 r$ & $\begin{array}{l}\text { Sagina procumbens recorded from BI: II- } \\
\text { IV, VIIb (Godwin 1975) }\end{array}$ \\
\hline Sagina sp. & RIA: $311+$ VA: $304+307 \mathrm{c} 334 \mathrm{c}$ & \\
\hline $\begin{array}{l}\text { Salicornia europaea L. (S. } \\
\text { brachystachya (G. F. W. } \\
\text { Meyer) D. König) }\end{array}$ & RIA: $301+311 \mathrm{c}$ VA: $307 \mathrm{c}$ & $\begin{array}{l}\text { BRD: 1st-2nd century A.D. (Körber- } \\
\text { Grohne 1967) NL: 3400-3300 B.C. (Zeist \& } \\
\text { Palfenier-Vegter 1981) }\end{array}$ \\
\hline Salix cf. arbuscula L. & II: 706ru 714r 720r & \\
\hline Salix aurita $\mathrm{L}$. & VA: $304 r$ 334r & BI: V-VI, VIIb/VIII (Godwin 1975) \\
\hline Salix caprea $\mathbf{L}$. & V: 165 r VI, VII: 165 r 711r 165r VA: $304 r$ & \\
\hline Salix cf. caprea $\mathbf{L}$. & $\begin{array}{l}\text { II: } 508 \mathrm{r} \text { IV: } 508+\mathrm{u} \text { V: } 508+\mathrm{u} \text { VI, VII: } 508+ \\
\text { 508+u VA: } 334 \mathrm{r}\end{array}$ & \\
\hline Salix cinerea $\mathrm{L}$. & $\begin{array}{l}\text { IV: } 209 \mathrm{c} 418 \mathrm{ru} \text { VI, VII: } 422 \mathrm{cu} \text { VIII: 513ru } \\
\text { LMA: } 522 \mathrm{c}\end{array}$ & \\
\hline Salix cf. cinerea $\mathbf{L}$. & II: $508+$ & \\
\hline Salix herbacea L. & $\begin{array}{l}\text { I: } 113 \mathrm{r} 714 \mathrm{r} 720 \mathrm{c} \text { III: } 158+\mathrm{u} 237 \mathrm{r} 714 \mathrm{r} \\
720 \mathrm{c}\end{array}$ & BI: I-IV (Godwin 1975) \\
\hline Salix cf. herbacea L. & III: 161ru & \\
\hline Salix phylicifolia L. & I: $513 \mathrm{r}$ & BI: I-IV (Godwin 1975) \\
\hline Salix cf. phylicifolia L. & $\begin{array}{l}\text { I: } 510+511+\text { III: } 152+\text { u } 153 \text { ru } 415 r u 416 r u \\
417 \text { ru } 503 \text { ru } 508+510 \mathrm{c} 511 \mathrm{c} 714 \mathrm{r}\end{array}$ & \\
\hline Salix polaris Wahlenb. & $\begin{array}{l}\text { I: 416cu 417ru 510c 511c 513r 545ru 548+u } \\
714 \text { c } 719+720 \text { c III: } 152+\text { u 209r 237r 415ru } \\
\text { 417ru 419ru 423ru 424+u 425ru 503r 506r } \\
507+\text { u 508+509r 510r 511r 701cu 704ru } \\
705 \text { r 706ru 714r 719+u 720c IV: } 238+u \text { V: } \\
238+u\end{array}$ & BI: Late Weichselian (Godwin 1975) \\
\hline Salix cf. polaris Wahlenb. & I: $113+$ III: $113 \mathrm{r} 512 \mathrm{ru}$ & \\
\hline Salix repens $\mathrm{L}$. & RIA: $107+$ & $\begin{array}{l}\text { BI: Late Weichselian, VIIb-VIII (Godwin } \\
\text { 1975) }\end{array}$ \\
\hline Salix reticulata $\mathrm{L}$. & $\begin{array}{l}\text { I: } 510 \mathrm{r} 511 \mathrm{r} 720 \mathrm{c} \text { II: } 546 \mathrm{ru} 554 \mathrm{ru} \text { III: } 153 \mathrm{ru} \\
\text { 415ru 416ru 417ru 419ru 506+u 508+510r } \\
\text { 511r 516ru 523r 701cu 706ru 714r 720c }\end{array}$ & BI: Late Weichselian (Godwin 1975) \\
\hline Salix sp. & $\begin{array}{l}\text { I: 313r 319r II: 523r III: 161ru 423ru 523r V: } \\
\text { 506ru 603ru VI, VII: 529r 537r 944r 944r VIII: } \\
\text { 328r 559ru }\end{array}$ & \\
\hline Salvia pratensis $\mathbf{L}$. & 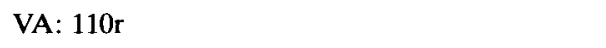 & \\
\hline
\end{tabular}


Table 2 continued

\begin{tabular}{|c|c|c|}
\hline Species & $\begin{array}{l}\text { Period, site number (cf. Table } 1 \text { ) and number } \\
\text { of macrofossils reported }\end{array}$ & $\begin{array}{l}\text { Reports on finds from neighbour countries. } \\
\text { Pollen zones and other periods are presented } \\
\text { as published by the authors cited }\end{array}$ \\
\hline Sambucus nigra L. & $\begin{array}{l}\text { GIA: } 301+\text { VA: } 172 \text { r } 301+304 \text { r } 306 \text { r } 307+ \\
312+334+427+543 \text { r EMA: } 172 \text { r } 245+427+ \\
428 \text { c } 429+430 \text { c } 431+521+\text { u } 522+\text { LMA: } \\
217+\text { u } 240 \text { r } 241+427+428+430 \mathrm{c} 521 \mathrm{cu} \\
522+552 \text { r }\end{array}$ & $\begin{array}{l}\text { BI: V, VIIa-VIII (Godwin 1975), } 3360 \pm 80 \\
\text { B.P. (Peglar \& Wilson 1978), } 2130 \pm 100 \\
\text { B.P. (Greig 1979b), Late 2nd century A.D. } \\
\text { (Wilson 1979), c. } 300 \text { A.D. (Wilson 1978), } \\
\text { 4th-5th century A.D. (Greig 1976) BRD: } \\
\text { Neolithic (Averdieck 1980, Kroll 1981), 12-8 } \\
\text { B.C. (Kučan 1981), 1st century A.D. } \\
\text { (Knörzer 1970), c. } 200 \text { A.D. (Körber- } \\
\text { Grohne 1979b) P: Hallstatt and later (Gluza } \\
\text { \& Wasylikowa 1977) }\end{array}$ \\
\hline Sambucus cf. nigra $\mathbf{L}$. & EMA: $564+$ LMA: $564+$ & \\
\hline Samolus valerandi $\mathrm{L}$. & VA: 334r EMA: 431+ & BI: 10th century A.D. (Wilson 1975) \\
\hline Saponaria officinalis L. & VA: $301 \mathrm{r}$ & $\begin{array}{l}\text { BRD: 1st century A.D. (Knörzer 1967a, } \\
\text { 1970) P: 11th-12th century A.D. } \\
\text { (Wasylikowa 1978) }\end{array}$ \\
\hline Saxifraga oppositifolia L. & I: 513r III: 416ru 417ru 503ru & BI: I/II, III (Godwin 1975) \\
\hline Scheuchzeria palustris $\mathrm{L}$. & $\begin{array}{l}\text { VI, VII: } 164 \mathrm{r} 175 \mathrm{c} 508 \mathrm{cu} \text { VIII: } 508+\mathrm{u} 513 \mathrm{ru} \\
559+826+\text { IX: } 170 \mathrm{r} 176 \mathrm{c} 501+502+513 \mathrm{c} \\
559+\end{array}$ & BI: VI-VIII (Godwin 1975) \\
\hline $\begin{array}{l}\text { Scirpus cespitosus L. } \\
\text { (Tricophorum cespitosum } \\
\text { (L.) Hartman) }\end{array}$ & VIII: 944r IX: 162r 164r 944r GIA: 244+ & BI: III, VI-VIII (Godwin 1975) \\
\hline $\begin{array}{l}\text { Scirpus lacustris L. } \\
\text { (Schoenoplectus lacustris } \\
\text { (L.) Palla) }\end{array}$ & $\begin{array}{l}\text { III: 417ru 540r 905r 995r IV: 104+ 238ru 905r } \\
\text { 995r V: 104r 213r 221r 238ru 529c 534c 555r } \\
\text { 603ru VI, VII: 213r 221r 410r 513ru 529r 534c } \\
\text { 549c 603ru 905r 410r 422cu 603ru 905r 944r } \\
\text { VIII: 325r 410r 531c 543r IX: 156r 157c 325r } \\
\text { 328+ 513r RIA: 301+ VA: 334+ EMA: 428r } \\
\text { 521ru LMA: } 217 c u \text { 428c 522r }\end{array}$ & \\
\hline $\begin{array}{l}\text { Scirpus lacustris L. subsp. } \\
\text { tabernaemontani (C. C. } \\
\text { Gmelin) Syme in Sowerby } \\
\text { (S. glaucus Sm., non Lam., } \\
\text { S. tabernaemontani C. C. } \\
\text { Gmelin) }\end{array}$ & $\begin{array}{l}\text { V: 104r 235ru VI, VII: 321+ 520+u VIII: } \\
\text { 543r IX: 327r 332c PRIA: 301r RIA: 301+ } \\
\text { 306r 311+ GIA: 242+ 301r 553r VA: 103c } \\
\text { 172r 304c 307c 334c EMA: 431+ }\end{array}$ & BI: II-V, VIIa-VIII (Godwin 1975) \\
\hline $\begin{array}{l}\text { Scirpus cf. lacustris L. subsp. } \\
\text { tabernaemontani (C. C. } \\
\text { Gmelin) Syme in Sowerby }\end{array}$ & IX: $557 \mathrm{r}$ & \\
\hline Scirpus maritimus L. & $\begin{array}{l}\text { VIII: 322r 323r IX: 323r 325+ 328r RIA: 301r } \\
\text { 306r 311r GIA: } 243+301 \text { r VA: } 301+307 \mathrm{c} \\
\text { EMA: 521ru LMA: 521ru }\end{array}$ & $\begin{array}{l}\text { BI: VIIa-VIII (Godwin 1975) NL: } 3400- \\
3300 \text { B.C. (Zeist \& Palfenier-Vegter 1981) } \\
\text { N: c. } 4000 \text { B.P. (Griffin 1980) }\end{array}$ \\
\hline Scirpus setaceus L. & GIA: 244+ VA: 202r EMA: 428r LMA: 428r & $\begin{array}{l}\text { BI: VIIb-VIII (Godwin 1975), 100-300 } \\
\text { A.D. (Wilson 1968), c. } 300 \text { A.D. (Wilson } \\
\text { 1978) }\end{array}$ \\
\hline Scirpus sylvaticus L. & $\begin{array}{l}\text { I: 510c 511c VI, VII: 422+u RIA: 311r GIA: } \\
\text { 244+ VA: 304c 334c LMA: 241c 430+ }\end{array}$ & \\
\hline Scirpus ef. sylvaticus L. & IV: 238ru V: 238ru & \\
\hline Scirpus sp. & $\begin{array}{l}\text { VI, VII: 529r 537r VIII: 559ru RIA: 306r } \\
\text { EMA: 172r 245c 521ru LMA: } 241+245+\end{array}$ & \\
\hline Scleranthus annuus L. & $\begin{array}{l}\text { VIII: } 214+702 \text { r PRIA: } 111+115 \text { r 301+ RIA: } \\
\text { 108r 301r VA: } 172+304 \text { c 307r 312r 334c } \\
\text { EMA: } 172+428 r \text { r } 429+\text { LMA: } 218 \text { r }\end{array}$ & \\
\hline Scleranthus cf. annuus L. & RIA: $107 \mathrm{c}$ & \\
\hline
\end{tabular}


Table 2 continued

\begin{tabular}{|c|c|c|}
\hline Species & $\begin{array}{l}\text { Period, site number (cf. Table 1) and number } \\
\text { of macrofossils reported }\end{array}$ & $\begin{array}{l}\text { Reports on finds from neighbour countries. } \\
\text { Pollen zones and other periods are presented } \\
\text { as published by the authors cited }\end{array}$ \\
\hline Scleranthus sp. & PRIA: 109r RIA: 101r GIA: 242r EMA: 245+ & \\
\hline Scutellaria galericulata $\mathbf{L}$. & RIA: 311r VA: 334+ & $\begin{array}{l}\text { NL: } 600-400 \text { B.C. (Zeist 1974) BRD: 1st } \\
\text { century A.D. (Knörzer 1970), 1st-2nd } \\
\text { century A.D. (Körber-Grohne 1967, 1979b) }\end{array}$ \\
\hline Secale cereale $\mathrm{L}$. & $\begin{array}{l}\text { IX: 931r 935r 1087r PRIA: 115r RIA: 108c } \\
\text { 125r 807r 935r GIA: 205c 228c 301c 712+ } \\
\text { 713+ 1087r VA: 103c 110c 202c 301c 304c 307r } \\
\text { 312c 320r 334c 984cu 985c 1065+ 1080+ } \\
\text { 1081+ 1097+ EMA: 709c 710c 992c LMA: } \\
218+406 r\end{array}$ & $\begin{array}{l}\text { BI: Pre-Roman Iron Age and later (Helbæk } \\
\text { 1952c, Godwin 1975) NL: 2nd century B.C. } \\
\text { (Zeist 1981) BRD: c. } 400 \text { B.C., 150-260 } \\
\text { A.D. (Körber-Grohne \& Piening 1979) P: } \\
\text { Neolithic and later (Gluza \& Wasylikowa } \\
\text { 1977) }\end{array}$ \\
\hline Secale sp. & GIA: $301+$ VA: $301+$ & \\
\hline Senecio jacobaea $\mathrm{L}$. & VA: $304+334 c$ & \\
\hline Senecio sp. & VIII: $425+$ & $\begin{array}{l}\text { Senecio congestus (R. Br.) DC. recorded } \\
\text { from NL: } 3230 \pm 35 \text { B.P. (Pals et al. 1980) }\end{array}$ \\
\hline Setaria italica (L.) Beauv. & RIA: 708 r GIA: $712 \mathrm{c} 713+$ & BRD: Hallstatt (Knörzer 1971c) \\
\hline $\begin{array}{l}\text { Setaria pumila (Poiret) } \\
\text { Schultes in Schultes \& } \\
\text { Schultes fil. (Setaria glauca, } \\
\text { auct., non (L.) Beauv.) }\end{array}$ & VA: $334+$ EMA: $521+u$ & $\begin{array}{l}\text { DDR: 7th-9th century A.D. (Lange 1976) } \\
\text { NL: } 510 \pm 65 \text { B.C. (Zeist 1968) P: Hallstatt } \\
\text { and later (Gluza \& Wasylikowa 1977), 9th-- } \\
\text { 12th century A.D. (Wasylikowa 1978) }\end{array}$ \\
\hline Setaria viridis (L.) Beauv. & $\begin{array}{l}\text { PRIA: 115r RIA: 708r GIA: } 712+\text { VA: } 304+ \\
\text { 334+ EMA: 521ru LMA: } 406+\end{array}$ & $\begin{array}{l}\text { NL: } 510 \pm 65 \text { B.C. (Zeist 1968) P: Hallstatt } \\
\text { and later (Gluza \& Wasylikowa 1977) }\end{array}$ \\
\hline $\begin{array}{l}\text { Silene alba (Miller) E. H. L. } \\
\text { Krause in Sturm } \\
\text { (Melandrium album (Miller) } \\
\text { Garcke) }\end{array}$ & $\begin{array}{l}\text { VIII: } 609+\text { PRIA: 301r RIA: 301r 306+ GIA: } \\
\text { 301r VA: } 110+301 \text { r 304+ 307r 334+ EMA: } \\
\text { 521ru LMA: 218r 406r 521ru }\end{array}$ & BI: VIIb-VIII (Godwin 1975) \\
\hline $\begin{array}{l}\text { Silene dioica (L.) Clairv. } \\
\text { (Melandrium rubrum } \\
\text { (Weigel) Garcke) }\end{array}$ & VA: 304 r $334+$ & $\begin{array}{l}\text { BI: I-III, VI-VIIb (Godwin 1975), 4th-5th } \\
\text { century A.D. (Greig 1976) BRD: 10th-12th } \\
\text { century A.D. (Willerding 1979b) }\end{array}$ \\
\hline Silene noctiflora $\mathbf{L}$. & VIII: 425r RIA: $311+$ VA: $334+$ & \\
\hline $\begin{array}{l}\text { Silene vulgaris (Moench) } \\
\text { Garcke (Silene cucubalus } \\
\text { Wib.) }\end{array}$ & VA: 307 r $985+$ LMA: $241+521+u$ & $\begin{array}{l}\text { BI: Late Weichselian, Roman Period } \\
\text { (Godwin 1975), 2nd century A.D. (Wilson } \\
\text { 1979) BRD: c. } 200 \text { A.D. (Körber-Grohne } \\
\text { 1979b) N: Late Viking - Early Medieval } \\
\text { (Tallantire 1979) }\end{array}$ \\
\hline $\begin{array}{l}\text { Silene cf. vulgaris (Moench) } \\
\text { Garcke }\end{array}$ & EMA: 172 r $429+$ & \\
\hline $\begin{array}{l}\text { Silene sp. (incl. Melandrium } \\
\text { sp.) }\end{array}$ & GIA: 712r VA: 304r EMA: 245+ & \\
\hline Sinapis arvensis $\mathrm{L}$. & $\begin{array}{l}\text { VIII: } 214+\text { RIA: } 108 \mathrm{r} 306 \mathrm{r} 311 \mathrm{r} 708 \mathrm{r} \text { GIA: } \\
\text { 228r 306r VA: } 110+307 \mathrm{c} 312 \mathrm{r} 334+985 \mathrm{c} \\
\text { EMA: 521ru LMA: } 406 \mathrm{r} 430+521 \mathrm{cu} 809 \mathrm{c}\end{array}$ & BI: VIIb-VIII (Godwin 1975) \\
\hline $\begin{array}{l}\text { Sisymbrium officinale (L.) } \\
\text { Scop. }\end{array}$ & RIA: 301r VA: 307r 334+ & $\begin{array}{l}\text { BI: } 3360 \pm 80 \text { B.P. (Peglar \& Wilson 1978), } \\
\text { Roman Period (Godwin 1975), 100-300 } \\
\text { A.D. (Wilson 1968) BRD: Neolithic } \\
\text { (Knörzer 1971a), Hallstatt (Knörzer 1974b) }\end{array}$ \\
\hline $\begin{array}{l}\text { Sium latifolium L. ( } \mathrm{S} \text {. } \\
\text { erectum Hudson) }\end{array}$ & VA: $307+334+$ & $\begin{array}{l}\text { BI: VIIa (Godwin 1975) NL: } 3400-3300 \\
\text { B.C. (Zeist \& Palfenier-Vegter 1981) BRD: } \\
\text { c. } 200 \text { A.D. (Körber-Grohne 1979b) }\end{array}$ \\
\hline Sium cf. latifolium $\mathbf{L}$. & EMA: $430+$ & \\
\hline Solanum dulcamara $\mathbf{L}$. & $\begin{array}{l}\text { IV: 209r VI, VII: 528ru 549+555r 520ru VIII: } \\
\text { 531r 603ru IX: 1036r RIA: 101r 306r 311r } \\
\text { GIA: 306r VA: 304+ 307+ 334+ EMA: 428r } \\
\text { 521ru LMA: } 217 \text { ru 521+u 522r }\end{array}$ & \\
\hline
\end{tabular}




\begin{tabular}{|c|c|c|}
\hline Species & $\begin{array}{l}\text { Period, site number (cf. Table 1) and number } \\
\text { of macrofossils reported }\end{array}$ & $\begin{array}{l}\text { Reports on finds from neighbour countries. } \\
\text { Pollen zones and other periods are presented } \\
\text { as published by the authors cited }\end{array}$ \\
\hline Solanum cf. dulcamara $\mathrm{L}$. & EMA: $430+431+$ LMA: $430 \mathrm{c}$ & \\
\hline $\begin{array}{l}\text { Solanum dulcamara } \mathrm{L} \text {. et } \mathrm{S} \text {. } \\
\text { nigrum } \mathrm{L} \text {. }\end{array}$ & EMA: $429+$ & \\
\hline Solanum nigrum $\mathbf{L}$. & $\begin{array}{l}\text { VIII: } 220+\text { PRIA: } 111 \mathrm{r} 115+301 \mathrm{r} \text { RIA: } 301 \mathrm{r} \\
\text { 306r 311r 708r GIA: } 243+244 \mathrm{c} \text { VA: } 202+ \\
\text { 301r 304r 307+ 334c EMA: } 427+428 \mathrm{c} 431 \mathrm{c} \\
\text { 521cu LMA: } 241 \mathrm{c} 427 \mathrm{c} 428 \mathrm{c} 430 \mathrm{c} 521 \mathrm{cu} 985 \mathrm{c}\end{array}$ & $\begin{array}{l}\text { BI: IV/V, VII-VIII (Godwin 1975) NL: } \\
\text { 3400-3300 B.C. (Zeist \& Palfenier-Vegter } \\
\text { 1981) }\end{array}$ \\
\hline Solanum cf. nigrum $\mathrm{L}$. & GIA: $242+$ VA: $172+$ EMA: $172+$ & \\
\hline Solanum sp. & $\begin{array}{l}\text { RIA: } 311 \text { r GIA: } 244+\text { EMA: } 172+245 \text { c } 428+ \\
\text { LMA: } 245+427+428+430 \text { c } 522 \text { r }\end{array}$ & \\
\hline Solidago virgaurea $\mathrm{L}$. & EMA: $430+$ & \\
\hline Sonchus arvensis $\mathbf{L}$ : & $\begin{array}{l}\text { RIA: 306r 311c GIA: 242r 244+ 301r VA: } \\
\text { 307c 334+ EMA: } 428+521+u \text { LMA: } 218 r \\
430+521 \text { ru }\end{array}$ & $\begin{array}{l}\text { BI: II/III, Roman Period (Godwin 1975) } \\
\text { NL: } 1195 \pm 50 \text { B.P. (Geel et al. 1983) BRD: } \\
\text { 12-8 B.C. (Kučan 1981) }\end{array}$ \\
\hline Sonchus asper (L.) Hill & $\begin{array}{l}\text { PRIA: } 115+\text { RIA: } 301 r \text { } 306+311 \text { c GIA: } 301 r \\
\text { VA: } 172+301+304+307 \mathrm{c} 334 \mathrm{c} 985 \text { r EMA: } \\
\text { 172+ } 245 \mathrm{c} 428+430+431+521+\text { u LMA: } \\
430 \mathrm{c}\end{array}$ & $\begin{array}{l}\text { BI: } 3360 \pm 80 \text { B.P. (Peglar \& Wilson 1978) } \\
\text { NL: } 3400-3300 \text { B.C. (Zeist \& Palfenier- } \\
\text { Vegter 1981) BRD: 12-8 B.C. (Kučan 1981) } \\
\text { P: Hallstatt and later (Gluza \& Wasylikowa } \\
\text { 1977) }\end{array}$ \\
\hline Sonchus oleraceus L. & $\begin{array}{l}\text { VIII: } 127 \mathrm{r} \text { RIA: } 108 \mathrm{r} 306+311 \mathrm{c} \text { VA: } 307 \mathrm{c} \\
\text { EMA: } 245+428+429+521 \mathrm{ru} \text { LMA: } 430 \mathrm{c} \\
\text { 504r 521ru } 1025 \mathrm{r}\end{array}$ & BI: VI (Godwin 1975) \\
\hline Sonchus palustris $\mathrm{L}$. & VA: $306 \mathrm{r}$ & $\begin{array}{l}\text { BI: Roman Period (Godwin 1975) NL: } \\
\text { 3400-3300 B.C. (Zeist \& Palfenier-Vegter } \\
\text { 1981) BRD: 12-8 B.C. (Kučan 1981), 1st- } \\
\text { 2nd century A.D. (Körber-Grohne 1967) }\end{array}$ \\
\hline Sonchus sp. & $\begin{array}{l}\text { GIA: } 242 \text { r } 244+\text { EMA: } 172+428 \text { r } 429+ \\
\text { LMA: } 241+430+\end{array}$ & \\
\hline Sorbus aucuparia L. & $\begin{array}{l}\text { VI, VII: } 508+u \text { VIII: } 513 \text { ru 603ru IX: } 513+ \\
\text { VA: } 304+312 \mathrm{c} 334 \mathrm{c}\end{array}$ & BI: II-IV, VI-VIII (Godwin 1975) \\
\hline $\begin{array}{l}\text { Sorbus cf. intermedia } \\
\text { (Ehrh.) Pers. }\end{array}$ & VIII: $987 \mathrm{r}$ & \\
\hline $\begin{array}{l}\text { Sparganium cf. angustifolium } \\
\text { Michx (S. affine Schnizlein) }\end{array}$ & II: $419+$ III: 417ru IV: 421 ru & $\begin{array}{l}\text { Sparganium angustifolium recorded from BI: } \\
\text { I-II, IV-VI (Godwin 1975) }\end{array}$ \\
\hline $\begin{array}{l}\text { Sparganium emersum } \\
\text { Rehmann (S. simplex } \\
\text { Hudson) }\end{array}$ & IX: 513r RIA: 101+ LMA: 521ru & BI: IV-VI, VIII (Godwin 1975) \\
\hline $\begin{array}{l}\text { Sparganium cf. emersum } \\
\text { Rehmann }\end{array}$ & VI, VII: 905r 905r VIII: 323r 905+ IX: $905+$ & \\
\hline $\begin{array}{l}\text { Sparganium erectum L. (S. } \\
\text { ramosum Hudson) }\end{array}$ & $\begin{array}{l}\text { VI, VII: 422ru RIA: } 101+\text { VA: } 334+\text { EMA: } \\
\text { 521ru LMA: } 521+u\end{array}$ & BI: II-III, VI, VIIb-VIII (Godwin 1975) \\
\hline Sparganium minimum Wallr. & $\begin{array}{l}\text { III: 161ru IV: 209r VI, VII: 549r IX: } 513 \text { r } \\
\text { RIA: } 101+\end{array}$ & \\
\hline $\begin{array}{l}\text { Sparganium cf. minimum } \\
\text { Wallr. }\end{array}$ & VI, VII: 501ru & . \\
\hline Sparganium sp. & $\begin{array}{l}\text { VI, VII: } 164 \mathrm{r} 422 \mathrm{ru} 906 \mathrm{r} \text { VIII: } 906+\text { IX: } 906+ \\
\text { LMA: } 521 \mathrm{ru}\end{array}$ & \\
\hline (to be continued) & $\begin{array}{l}\text { VIII: 127c PRIA: } 109+111 \mathrm{c} 114+115+141+ \\
\text { 148c 201c 301+ RIA: } 101 \mathrm{c} 105+107 \mathrm{c} 108 \mathrm{c} \\
\text { 139c } 151 \mathrm{c} 301+303 \mathrm{c} 308 \mathrm{c} 311 \mathrm{r} 315 \mathrm{r} \text { 708r GIA: } \\
205+228+242 \mathrm{r} 244+301 \mathrm{c} 712+\text { VA: } 103+\end{array}$ & $\begin{array}{l}\text { BI: VIIb-VIII (Godwin 1975) NL: } 1370 \pm 60 \\
\text { B.C. (Zeist 1968) }\end{array}$ \\
\hline
\end{tabular}




\begin{tabular}{|c|c|c|}
\hline Species & $\begin{array}{l}\text { Period, site number (cf. Table 1) and number } \\
\text { of macrofossils reported }\end{array}$ & $\begin{array}{l}\text { Reports on finds from neighbour countries. } \\
\text { Pollen zones and other periods are presented } \\
\text { as published by the authors cited }\end{array}$ \\
\hline $\begin{array}{l}\text { Spergula arvensis } \mathrm{L} \text {. } \\
\text { (continued) }\end{array}$ & $\begin{array}{l}172+202 \text { r 301c } 304+307 \text { c } 312 \text { r } 320+334 c \\
985+\text { EMA: } 172 \text { c } 245 \text { c } 427+428+429+430+ \\
431+521+\text { u } 992 \text { r LMA: } 241+245+427+ \\
430+521 \text { ru } 809 r\end{array}$ & \\
\hline Spergula sp. & VIII: 947r EMA: $245+$ & \\
\hline $\begin{array}{l}\text { Spergularia marina (L.) } \\
\text { Griseb. (S. salina I. \& C. } \\
\text { Presl) }\end{array}$ & RIA: $311 \mathrm{c}$ VA: $307 \mathrm{c}$ & $\begin{array}{l}\text { BRD: 1st-2nd century A.D. (Körber- } \\
\text { Grohne 1967) }\end{array}$ \\
\hline $\begin{array}{l}\text { Spergularia cf. marina (L.) } \\
\text { Griseb. }\end{array}$ & RIA: $301+$ & \\
\hline $\begin{array}{l}\text { Spergularia media (L.) C. } \\
\text { Presl (S. marginata (DC.) } \\
\text { Kittel) }\end{array}$ & RIA: $311 \mathrm{c}$ VA: $307 \mathrm{c}$ & $\begin{array}{l}\text { Spergularia media-type recorded from NL: } \\
600-400 \text { B.C. (Zeist 1974) }\end{array}$ \\
\hline Stachys cf. annua (L.) L. & VA: $172 \mathrm{r}$ & $\begin{array}{l}\text { Stachys annua recorded from BRD: 1st } \\
\text { century A.D. (Knörzer 1970), and from P: } \\
\text { 9th-12th century A.D. (Wasylikowa 1978) }\end{array}$ \\
\hline Stachys arvensis (L.) $\mathbf{L}$. & GIA: $712 \mathrm{r}$ & $\begin{array}{l}\text { BI: Roman Period (Godwin 1975) NL: } 500 \\
\text { B.C. - } 200 \text { A.D. (Zeist 1974) BRD: 2nd } \\
\text { century A.D. (Knörzer 1979b) DDR: Late } \\
\text { La Tène (Lange 1975) }\end{array}$ \\
\hline Stachys cf. arvensis (L.) L. & LMA: 406r & \\
\hline Stachys palustris $\mathrm{L}$. & $\begin{array}{l}\text { VI, VII: 520ru RIA: 301r 306r 311+ GIA: } \\
\text { 301r VA: 301r 304r 307c 334+ 985r EMA: } \\
\text { 521cu LMA: 521ru 522r }\end{array}$ & $\begin{array}{l}\text { BI: Late Weichselian, VIIa-VIIb (Godwin } \\
\text { 1975) }\end{array}$ \\
\hline Stachys ef. palustris $\mathbf{L}$. & EMA: $428 r$ & \\
\hline Stachys sylvatica $L$. & $\begin{array}{l}\text { V: 421ru VI, VII: 421ru EMA: 564r LMA: } \\
\text { 564r }\end{array}$ & BI: IV, VIIa-VIII (Godwin 1975) \\
\hline Stachys sp. & EMA: $428+430+$ LMA: $427+$ & \\
\hline Staphylea pinnata $\mathrm{L}$. & RIA: $412 r$ & \\
\hline $\begin{array}{l}\text { Stellaria alsine Grimm (S. } \\
\text { uliginosa Murray) }\end{array}$ & RIA: 101+ EMA: 521ru & $\begin{array}{l}\text { BI: II-III, VIIb-VIII (Godwin 1975) BRD: } \\
\text { 1st century A.D. (Knörzer 1970), c. } 200 \\
\text { A.D. (Körber-Grohne 1979b) }\end{array}$ \\
\hline Stellaria graminea L. & $\begin{array}{l}\text { II: } 155 \text { r VIII: } 220+\text { PRIA: } 115 \text { r VA: } 103 \text { r } 307 r \\
\text { EMA: } 428+429 \text { c } 430+521+\text { u LMA: } 218 r \\
241+428 r 521+u\end{array}$ & BI: I, VIIb-VIII (Godwin 1975) \\
\hline Stellaria cf. graminea L. & VIII: $207 \mathrm{r} 219 \mathrm{r}$ & \\
\hline Stellaria media (L.) Vill. & $\begin{array}{l}\text { VIII: } 543 \mathrm{r} 609+1009+1010+\text { PRIA: } 109+ \\
111+114 \mathrm{r} 115+301+\text { RIA: } 101+107 \mathrm{r} 108+ \\
139+301 \mathrm{c} 306 \mathrm{r} 311 \mathrm{c} \text { GIA: } 242+243+244+ \\
301+712 \mathrm{c} 713+\text { VA: } 103 \mathrm{r} 172 \mathrm{c} 202+301+ \\
304 \mathrm{c} 307 \mathrm{c} 312 \mathrm{r} 334 \mathrm{c} 427 \mathrm{c} 985+\text { EMA: } 172 \mathrm{c} \\
245 \mathrm{c} 427 \mathrm{c} 428 \mathrm{c} 429 \mathrm{c} 430+431 \mathrm{c} 521 \mathrm{cu} \text { LMA: } \\
171 \mathrm{r} 241+245+406+427 \mathrm{c} 428 \mathrm{c} 430+504+ \\
521 \mathrm{cu} 809 \mathrm{r} 1025+\end{array}$ & $\begin{array}{l}\text { BI: IV, VIIb-VIII (Godwin 1975) NL: } 3400 \\
\text { 3300 B.C. (Zeist \& Palfenier-Vegter 1981) } \\
\text { BRD: Neolithic (Kroll 1981) }\end{array}$ \\
\hline Stellaria cf. nemorum L. & VA: $304 \mathrm{r} 334 \mathrm{r}$ & $\begin{array}{l}\text { Stellaria nemorum recorded from BRD: } 900 \\
\text { B.C. }-200 \text { A.D. (Stalling 1983) }\end{array}$ \\
\hline Stellaria palustris Retz. & $\begin{array}{l}\text { RIA: } 306+311+\text { GIA: 306r VA: 202r 304+ } \\
\text { 307r 334c }\end{array}$ & $\begin{array}{l}\text { BI: IV/V, VIl/VIII (Godwin 1975) NL: } 2690 \\
\pm 60 \text { B.P. (Geel et al. 1983) BRD: 12-8 B.C. } \\
\text { (Kučan 1981) }\end{array}$ \\
\hline Stellaria cf. palustris Retz. & PRIA: 301r RIA: 301+ GIA: 301r VA: 301r & \\
\hline Stellaria sp. & IX: 328r & \\
\hline
\end{tabular}


Table 2 continued

\begin{tabular}{|c|c|c|}
\hline Species & $\begin{array}{l}\text { Period, site number (cf. Table 1) and number } \\
\text { of macrofossils reported }\end{array}$ & $\begin{array}{l}\text { Reports on finds from neighbour countries. } \\
\text { Pollen zones and other periods are presented } \\
\text { as published by the authors cited }\end{array}$ \\
\hline $\begin{array}{l}\text { Suaeda maritima (L.) } \\
\text { Dumort. }\end{array}$ & RIA: $301+306 r$ 311c VA: 307c 334r & $\begin{array}{l}\text { BI: IV/V-VIII (Godwin 1975) NL: } 600-400 \\
\text { B.C. (Zeist 1974) BRD: 1st-2nd century } \\
\text { A.D. (Körber-Grohne 1967) }\end{array}$ \\
\hline Symphytum officinale L. & VA: $307 \mathrm{r}$ & $\begin{array}{l}\text { BRD: 1st-2nd century A.D. (Körber- } \\
\text { Grohne 1967) P: 9th-12th century A.D. } \\
\text { (Wasylikowa 1978) }\end{array}$ \\
\hline $\begin{array}{l}\text { Taraxacum officinale-group } \\
\text { (T. vulgare Schrank) }\end{array}$ & $\begin{array}{l}\text { RIA: } 301+311 \text { r VA: 307c LMA: 171r 217ru } \\
\text { 245+ 521ru 1025+ }\end{array}$ & $\begin{array}{l}\text { Taraxacum sp. recorded from BI: II/III-IV, } \\
\text { Roman Period (Godwin 1975) BRD: 1st-2nd } \\
\text { century A.D. (Körber-Grohne 1967, 1979b) }\end{array}$ \\
\hline Taraxacum sp. & IV: $104+\mathrm{V}: 104 \mathrm{r}$ & \\
\hline Taxus baccata L. & VIII: $1096 r$ & $\begin{array}{l}\text { BI: VI-VIII (Godwin 1975) P: Mesolithic } \\
\text { (Gluza \& Wasylikowa 1977) }\end{array}$ \\
\hline Taxus sp. & VIII: $1057 \mathrm{r}$ & \\
\hline Thalictrum flavum L. & RIA: 311r VA: 307r & $\begin{array}{l}\text { BI: I-II, IV, VI-VIII (Godwin 1975) NL: } \\
500 \text { B.C. - } 200 \text { A.D. (Zeist 1974) BRD: 1st } \\
\text { century A.D. (Knörzer 1970), 1st-2nd } \\
\text { century A.D. (Körber-Grohne 1967) }\end{array}$ \\
\hline Thalictrum minus L. & RIA: $311 \mathrm{r}$ & $\begin{array}{l}\text { BI: Late Weichselian, VI/VII, VIIb (Godwin } \\
\text { 1975) }\end{array}$ \\
\hline Thlaspi arvense L. & $\begin{array}{l}\text { VIII: } 543+\text { PRIA: } 114 \text { r } 115 \text { r RIA: } 107 \text { r 301r } \\
\text { 306c 311+ VA: } 172+202 \text { r 301r 304+ 307c } \\
320+334 \text { c 985+ EMA: } 245+427+428+ \\
429+431+521 \text { cu LMA: } 218 \text { r } 241+406+ \\
427+428+521+\text { u } 522+\end{array}$ & BI: VIIb-VIII (Godwin 1975) \\
\hline Thymus serpyllum L. & VA: $304 r$ 334r & $\begin{array}{l}\text { BI: Late Weichselian (Godwin 1975) BRD: } \\
\text { 1st century A.D. (Knörzer 1967c, 1970), c. } \\
200 \text { A.D. (Körber-Grohne 1979b) }\end{array}$ \\
\hline $\begin{array}{l}\text { Tilia cordata Miller ( } \mathrm{T} \text {. } \\
\text { ulmifolia Scop.) }\end{array}$ & $\begin{array}{l}\text { VI, VII: } 410 \mathrm{c} 159+410 \mathrm{c} 422 \mathrm{cu} 501+502+ \\
508 \mathrm{cu} 513 \mathrm{cu} \text { VIII: } 410 \mathrm{c} 501+502+508+\mathrm{u} \\
\text { 513cu 559ru IX: } 502+513 \mathrm{c}\end{array}$ & $\begin{array}{l}\text { BRD: } 7200-5500 \text { B.C. and later (Stalling } \\
\text { 1983) P: Mesolithic (Gluza \& Wasylikowa } \\
\text { 1977) }\end{array}$ \\
\hline Tilia cf. cordata Miller & VI, VII: $528+u$ VIII: $317 \mathrm{r}$ & \\
\hline $\begin{array}{l}\text { Tilia } \times \text { vulgaris Hayne }(\mathrm{T} \text {. } \\
\text { europaea L. pro parte) }\end{array}$ & VI, VII: $551 \mathrm{cu}$ VIII: $551 \mathrm{cu}$ & BI: VIIa-VIII (Godwin 1975) \\
\hline $\begin{array}{l}\text { Torilis japonica (Houtt.) DC. } \\
\text { (T. anthriscus (L.) C.C. } \\
\text { Gmelin, non Gaertner) }\end{array}$ & VA: 304r 334+ EMA: 430+ 521ru LMA: 218r & $\begin{array}{l}\text { BI: VIIb-VIII (Godwin 1975), c. } 300 \text { A.D. } \\
\text { (Wilson 1978) BRD: 12-8 B.C. (Kučan } \\
\text { 1981), c. } 200 \text { A.D. (Körber-Grohne 1979b) } \\
\text { P: Hallstatt and later (Gluza \& Wasylikowa } \\
\text { 1977), 9th-12th century A.D. (Wasylikowa } \\
\text { 1978) }\end{array}$ \\
\hline Trapa natans L. & $\begin{array}{l}\text { VI, VII: } 826+905 \text { r 513cu 905+ 906r VIII: } \\
\text { 513cu 905r 906r IX: 513c 905r 906+ }\end{array}$ & $\begin{array}{l}\text { S: Late Glacial, Zones BO-2, AT-1, AT-2, } \\
\text { SB, SA (Björk \& Digerfeldt 1965) }\end{array}$ \\
\hline Trifolium arvense L. & RIA: $139+$ GIA: $712 \mathrm{r}$ & \\
\hline $\begin{array}{l}\text { Trifolium campestre Schreber } \\
\text { in Sturm }\end{array}$ & PRIA: $115 \mathrm{r}$ & $\begin{array}{l}\text { BI: VIIb (Godwin 1975) NL: } 500 \text { B.C. }-200 \\
\text { A.D. (Zeist 1974) BRD: c. } 200 \text { B.C. } \\
\text { (Knörzer 1979a), 12-8 B.C. (Kučan 1981) }\end{array}$ \\
\hline $\begin{array}{l}\text { Trifolium cf. campestre } \\
\text { Schreber in Sturm }\end{array}$ & EMA: $429+$ & \\
\hline Trifolium dubium Sibth. & PRIA: $115 \mathrm{r}$ & \\
\hline Trifolium cf. dubium Sibth. & VA: $103 r$ & \\
\hline Trifolium fragiferum $\mathrm{L}$. & VA: $307 c$ & \\
\hline
\end{tabular}


Table 2 continued

\begin{tabular}{|c|c|c|}
\hline Species & $\begin{array}{l}\text { Period, site number (cf. Table } 1 \text { ) and number } \\
\text { of macrofossils reported }\end{array}$ & $\begin{array}{l}\text { Reports on finds from neighbour countries. } \\
\text { Pollen zones and other periods are presented } \\
\text { as published by the authors cited }\end{array}$ \\
\hline Trifolium pratense $\mathrm{L}$. & RIA: 311c 708r EMA: 992r & $\begin{array}{l}\text { BI: Late 2nd century A.D. (Wilson 1979) } \\
\text { NL: } 500 \text { B.C. - } 200 \text { A.D. (Zeist 1974) BRD: } \\
\text { 2nd century A.D. (Knörzer 1979b) }\end{array}$ \\
\hline Trifolium cf. pratense L. & VA: $304 \mathrm{c} 334+$ & \\
\hline $\begin{array}{l}\text { Trifolium pratense } \mathrm{L} \text {. et } \mathbf{T} \text {. } \\
\text { repens } \mathrm{L} \text {. }\end{array}$ & RIA: $311+$ & \\
\hline Trifolium repens $\mathrm{L}$. & $\begin{array}{l}\text { RIA: 311c GIA: } 244+712 \text { r VA: } 304 \text { c } 307 \text { c } \\
\text { 334c EMA: } 429+\text { LMA: } 171+427+428+ \\
430+504+\end{array}$ & $\begin{array}{l}\text { BI: Roman Period (Dickson et al. 1979) NL: } \\
\text { 3400-3300 B.C. (Zeist \& Palfenier-Vegter } \\
\text { 1981) BRD: 12-8 B.C. (Kučan 1981), 1st- } \\
\text { 2nd century A.D. (Knörzer 1970, 1979b, } \\
\text { Körber-Grohne 1967) }\end{array}$ \\
\hline Trifolium sp. & RIA: 301r 311c GIA: 553+ VA: 307c & \\
\hline Triglochin maritima L. & $\begin{array}{l}\text { III: } 506+\text { u 507+u RIA: } 301 \text { r 311c VA: } 304+ \\
\text { 307c 334c EMA: } 522+\end{array}$ & \\
\hline Triglochin palustris $\mathrm{L}$. & RIA: $311+$ VA: $304 \mathrm{r} 307 \mathrm{c} 334+$ & NL: $370 \pm 70$ B.C. (Zeist 1974) \\
\hline Triticum aestivum $\mathrm{L}$. & $\begin{array}{l}\text { PRIA: } 301+\text { RIA: 106r VA: 301r 307r 312c } \\
\text { 320r 334c }\end{array}$ & $\begin{array}{l}\text { BRD: c. } 400 \text { B.C. (Körber-Grohne \& } \\
\text { Piening 1979) P: Neolithic, Bronze Age, } \\
\text { Hallstatt and later (Gluza \& Wasylikowa } \\
\text { 1977) S: Neolithic, Bronze Age and later } \\
\text { (Hjelmqvist 1979) }\end{array}$ \\
\hline $\begin{array}{l}\text { Triticum aestivum } L \text {. et } T \text {. } \\
\text { compactum Host }\end{array}$ & $\begin{array}{l}\text { VIII: } 142 r 203+204+227 \text { r } 233 \text { r } 236 r \text { r } 401+ \\
403+405+409+425+527 \text { r } 532 \text { r } 533 \text { r } 702 r \\
\text { 940r } 1032 \text { r GIA: } 713 \text { r VA: } 304 c\end{array}$ & $\begin{array}{l}\text { BI: VIIb-VIII (Godwin 1975), Neolithic, } \\
\text { Bronze Age (Jessen \& Helbæk 1944, } \\
\text { Helbæk 1952c, Godwin 1975) }\end{array}$ \\
\hline Triticum compactum Host & $\begin{array}{l}\text { VIII: } 606+607 \text { r } 703 \text { r 967r } 1007 \text { r RIA: } 106+ \\
\text { GIA: } 712+\end{array}$ & $\begin{array}{l}\text { BI: VIIb-VIII (Godwin 1975) BRD: } \\
\text { Neolithic (Bertsch 1954) P: Neolithic, } \\
\text { Bronze Age (Gluza \& Wasylikowa 1977) }\end{array}$ \\
\hline Triticum cf. compactum Host & VIII: $208+542+$ & \\
\hline Triticum dicoccon Schrank & $\begin{array}{l}\text { VIII: } 149 \mathrm{r} 203+204+208 \mathrm{c} 221 \mathrm{r} 227 \mathrm{r} 233 \mathrm{r} \\
\text { 236r 301c 317r 401c 403+404c 405c 409+ 413r } \\
505 \mathrm{r} 514 \mathrm{r} 515 \mathrm{r} 527 \mathrm{r} 532 \mathrm{r} 533 \mathrm{r} 541+542 \mathrm{c} 609 \mathrm{r} \\
702 \mathrm{c} 703+902 \mathrm{r} 909 \mathrm{r} 910 \mathrm{r} 922+925 \mathrm{r} 927 \mathrm{r} 928 \mathrm{r} \\
946 \mathrm{r} 947 \mathrm{r} 949+950+958+959+962 \mathrm{r} 964+ \\
965 \mathrm{c} 966 \mathrm{c} 971 \mathrm{r} 974+976+977 \mathrm{r} 978 \mathrm{c} 979 \mathrm{r} \\
986+987 \mathrm{r} 996 \mathrm{r} 997 \mathrm{r} 1000 \mathrm{r} 1001 \mathrm{r} 1006 \mathrm{r} 1008+ \\
1020+1021 \mathrm{r} 1031+1032+1034+1057 \mathrm{r} 1064 \mathrm{r} \\
1065+1069+1072 \mathrm{r} 1073 \mathrm{r} 1074 \mathrm{r} 1084 \mathrm{r} 1086+ \\
1089 \mathrm{c} 1096 \mathrm{r} \text { IX: } 935 \mathrm{r} \text { PRIA: } 115+301 \mathrm{r} 951 \mathrm{r} \\
1065 \mathrm{r} \text { RIA: } 106 \mathrm{r} 212 \mathrm{r} 301+707 \mathrm{c} 708 \mathrm{c} 804 \mathrm{ru} \\
\text { 935+936+u 1065ru VA: } 301 \mathrm{r} 1065 \mathrm{r}\end{array}$ & $\begin{array}{l}\text { BI: VIIb-VIII (Godwin 1975) NL: 3400- } \\
\text { 3300 B.C. (Zeist \& Palfenier-Vegter 1981) } \\
\text { BRD: Band Ceramic (Knörzer 1974a) P: } \\
\text { Neolithic, Bronze Age (Gluza \& Wasylikowa } \\
\text { 1977) }\end{array}$ \\
\hline $\begin{array}{l}\text { Triticum cf. dicoccon } \\
\text { Schrank }\end{array}$ & VIII: 938 r 939r 940r 1001r 1003r VA: $1065 r$ & \\
\hline $\begin{array}{l}\text { Triticum dicoccon Schrank et } \\
\text { T. monococcum L. }\end{array}$ & $\begin{array}{l}\text { VIII: } 208 \mathrm{c} 405 \mathrm{c} 409+702+922+938 \mathrm{r} 939 \mathrm{r} \\
940 \mathrm{r} 946 \mathrm{r} 964 \mathrm{r} 965+966+971 \mathrm{r} 974 \mathrm{r} 976+ \\
978 \mathrm{c} 986+987 \mathrm{r} 1000+1001+1031 \mathrm{r} 1032 \mathrm{r} \\
1034+1056 \mathrm{r} 1064 \mathrm{r} 1065 \mathrm{r} 1069 \mathrm{r} 1074+1084 \mathrm{r} \\
1086 \mathrm{r}\end{array}$ & \\
\hline $\begin{array}{l}\text { Triticum dicoccon Schrank et } \\
\text { T. spelta L. }\end{array}$ & VIII: $425 \mathrm{c} 958+959+$ & \\
\hline (to be continued) & $\begin{array}{l}\text { VIII: } 150 \mathrm{r} 203+204+208 \mathrm{c} 223 \mathrm{r} 224 \mathrm{r} 227 \mathrm{r} \\
233 \mathrm{r} 403+405+409 \mathrm{r} 542 \mathrm{c} 609 \mathrm{r} 702+703+ \\
716 \mathrm{r} 909 \mathrm{r} 910 \mathrm{r} 922+925+946+947 \mathrm{r} 953 \mathrm{r} \\
954 \mathrm{r} 956 \mathrm{r} 958 \mathrm{r} 959+962+963 \mathrm{r} 964+965 \mathrm{c} \\
966 \mathrm{c} 968 \mathrm{r} 971+974+975 \mathrm{r} 976+978 \mathrm{c} 981 \mathrm{r} \\
982 \mathrm{r} 986+987+1000+1001 \mathrm{r} 1003 \mathrm{r} 1004+\end{array}$ & $\begin{array}{l}\text { BI: VIIb-VIII (Godwin 1975) BRD: } \\
\text { Neolithic (Bertsch 1954, Knörzer 1971a, } \\
\text { Kroll 1981), Hallstatt (Knörzer 1971c) P: } \\
\text { Neolithic, Bronze Age (Gluza \& Wasylikowa } \\
\text { 1977) }\end{array}$ \\
\hline
\end{tabular}


Table 2 continued

\begin{tabular}{|c|c|c|}
\hline Species & $\begin{array}{l}\text { Period, site number (cf. Table 1) and number } \\
\text { of macrofossils reported }\end{array}$ & $\begin{array}{l}\text { Reports on finds from neighbour countries. } \\
\text { Pollen zones and other periods are presented } \\
\text { as published by the authors cited }\end{array}$ \\
\hline $\begin{array}{l}\text { Triticum monococcum L. } \\
\text { (continued) }\end{array}$ & $\begin{array}{l}1005+1012 \text { r } 1013 \mathrm{r} 1014 \mathrm{r} 1020 \mathrm{r} 1028 \mathrm{r} 1029 \mathrm{r} \\
\text { 1030r 1034+1056r 1064r } 1065+1069+1074+ \\
\text { 1084+1086+1089+1093r 1095r PRIA: } \\
1065+\text { RIA: } 1065 \mathrm{r}\end{array}$ & \\
\hline Triticum cf. monococcum $\mathbf{L}$. & VIII: $317 \mathrm{r}$ & \\
\hline Triticum spelta $L$. & $\begin{array}{l}\text { VIII: } 116 \mathrm{c} 203+204+403+922 \mathrm{c} 923 \mathrm{c} 958+ \\
\text { 959+ IX: } 993 \text { r PRIA: } 115+\text { RIA: } 993 \mathrm{r} 994 \mathrm{ru}\end{array}$ & $\begin{array}{l}\text { BI: VIIb-VIII (Godwin 1975) BRD: } \\
\text { Neolithic (Bertsch 1954) P: Neolithic (Gluza } \\
\text { \& Wasylikowa 1977) }\end{array}$ \\
\hline Triticum cf. spelta L. & VIII: $409 \mathrm{r}$ & \\
\hline Triticum sp. & 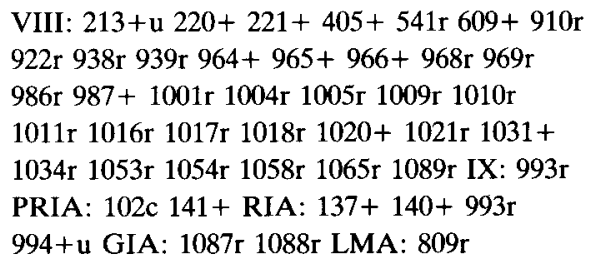 & \\
\hline Typha sp. & VI, VII: 422ru VA: 307r & BI: I-II, IV, VI, VIIb-VIII (Godwin 1975) \\
\hline Ulmus glabra Hudson & V: $539+$ VI, VII: $159 \mathrm{r}$ & $\begin{array}{l}\text { P: Mesolithic, Iron Age (Gluza \& } \\
\text { Wasylikowa 1977) }\end{array}$ \\
\hline Ulmus cf. glabra Hudson & EMA: $172+$ & \\
\hline Urtica dioica $\mathrm{L}$. & $\begin{array}{l}\text { II: 523c 538r V: 535r VI, VII: 549c VIII: } \\
\text { 603ru PRIA: 109+ 301c RIA: 301c 306r 311c } \\
\text { GIA: } 242+244 \mathrm{c} 301+\text { VA: } 172 \mathrm{c} 202 \mathrm{c} 301 \mathrm{c} \\
\text { 304+ 307c 334c 427c EMA: 172c 245+427c } \\
\text { 428c 429c 430c 431+ 521cu 564r LMA: 241c } \\
\text { 427c 428c 429+430c 521cu 552r 564r 1025r }\end{array}$ & BI: I-VI, VIIb-VIII (Godwin 1975) \\
\hline Urtica urens $\mathbf{L}$. & $\begin{array}{l}\text { PRIA: 109r RIA: 306r 311c GIA: } 242+243+ \\
244+\text { VA: } 172 \mathrm{c} 202+301 \mathrm{r} 304 \mathrm{c} 307 \mathrm{c} 334 \mathrm{c} \\
\text { EMA: } 172 \mathrm{c} 245 \mathrm{c} 427 \mathrm{c} 428 \mathrm{c} 429 \mathrm{c} 431 \mathrm{c} 521 \mathrm{cu} \\
\text { LMA: } 241 \mathrm{c} 245+427 \mathrm{c} 428+430+521 \mathrm{cu}\end{array}$ & $\begin{array}{l}\text { BI: VIIb-VIII (Godwin 1975) NL: } 1000 \\
\text { B.C. (Pals 1977) BRD: 12-8 B.C. (Kučan } \\
\text { 1981) DDR: Late La Têne (Lange 1975) }\end{array}$ \\
\hline Utricularia sp. & VI, VII: $944 \mathrm{r}$ & \\
\hline Vaccinium myrtillus $\mathrm{L}$. & IX: 501r & BI: III, VIII (Godwin 1975) \\
\hline Vaccinium cf. myrtillus $L$. & RIA: $311+$ VA: $312 \mathrm{c} 334 \mathrm{c}$ & \\
\hline $\begin{array}{l}\text { Vaccinium oxycoccos } \mathrm{L} \text {. } \\
\text { (Oxycoccus quadripetalus } \\
\text { Br.-Bl., O. palustris Pers.) }\end{array}$ & $\begin{array}{l}\text { VI, VII: } 501+u \text { u } 175 c 501+508+u \text { VIII: } 127 \text { r } \\
180+501+508+u \text { IX: } 176 \mathrm{c} 332+501+513+ \\
\text { RIA: } 301+602 c\end{array}$ & BI: II-IV, VIIa-VIII (Godwin 1975) \\
\hline Vaccinium uliginosum $\mathrm{L}$. & RIA: $128 \mathrm{r}$ & \\
\hline $\begin{array}{l}\text { Vaccinium uliginosum L. } \\
\text { subsp. microphyllum Lange }\end{array}$ & $\begin{array}{l}\text { II: } 416+\mathrm{u} 417 \mathrm{r} \text { III: } 416 \mathrm{ru} 417 \mathrm{ru} \text { IV: } 235 \mathrm{cu} \text { VI, } \\
\text { VII: } 501 \mathrm{ru}\end{array}$ & \\
\hline Vaccinium vitis-idaea $\mathrm{L}$. & RIA: $107+$ EMA: $521+u$ LMA: $521+u$ & BI: V-VI, VIIb (Godwin 1975) \\
\hline Vaccinium cf. vitis-idaea $L$. & LMA: $406+$ & \\
\hline Vaccinium sp. & VA: $304+$ & \\
\hline Valeriana officinalis L. & VA: $334 \mathrm{r}$ & $\begin{array}{l}\text { BI: II, VIIb-VIII (Godwin 1975) NL: } 200 \\
\text { B.C. (Zeist 1981) BRD: c. } 200 \text { A.D. } \\
\text { (Körber-Grohne 1979b) P: Hallstatt (Gluza } \\
\text { \& Wasylikowa 1977), first half 11th century } \\
\text { A.D. (Kosina 1978) }\end{array}$ \\
\hline $\begin{array}{l}\text { Valeriana officinalis L. } \\
\text { subsp. sambucifolia (Mikan } \\
\text { fil.) Celak. (V. sambucifolia } \\
\text { Mikan fil.) }\end{array}$ & VIII: $531 \mathrm{r}$ & $\begin{array}{l}\text { Valeriana officinalis (incl. V. sambucifolia) } \\
\text { recorded from BI: II, VIIb-VIII (Godwin } \\
\text { 1975). V.o. subsp. sambucifolia recorded } \\
\text { from N:Late Viking (Tallantire 1979) }\end{array}$ \\
\hline
\end{tabular}




\begin{tabular}{|c|c|c|}
\hline Species & $\begin{array}{l}\text { Period, site number (cf. Table 1) and number } \\
\text { of macrofossils reported }\end{array}$ & $\begin{array}{l}\text { Reports on finds from neighbour countries. } \\
\text { Pollen zones and other periods are presented } \\
\text { as published by the authors cited }\end{array}$ \\
\hline Verbascum cf. nigrum $\mathbf{L}$. & PRIA: 301r RIA: 301r & \\
\hline Verbascum thapsus $\mathrm{L}$. & LMA: $171 \mathrm{r}$ & \\
\hline Verbascum sp. & EMA: $427+$ & \\
\hline Verbena officinalis $\mathbf{L}$. & EMA: 430c LMA: $430+$ & $\begin{array}{l}\text { BI: Roman Period (Godwin 1975) BRD: 1st } \\
\text { century A.D. (Knörzer 1967b, 1967c, 1970, } \\
\text { Lange 1975) DDR: 7th-9th century A.D. } \\
\text { (Lange 1976) P: 9th-12th century A.D. } \\
\text { (Wasylikowa 1978), Early Medieval (Gluza } \\
\text { \& Wasylikowa 1977) }\end{array}$ \\
\hline Veronica arvensis $\mathbf{L}$. & PRIA: 301r RIA: 301r & \\
\hline Veronica chamaedrys L. & GIA: $712 \mathrm{r}$ & $\begin{array}{l}\text { BRD: 1st century A.D. (Knörzer 1970), 2nd } \\
\text { century A.D. (Knörzer 1979b, Körber- } \\
\text { Grohne 1979b) }\end{array}$ \\
\hline Veronica cf. opaca Fries & VA: $301 r$ & \\
\hline Veronica polita Fries & PRIA: $111+$ GIA: $712+$ & \\
\hline Veronica scutellata L. & RIA: 301c VA: 301r & BRD: 1st century A.D. (Knörzer 1970) \\
\hline Veronica serpyllifolia L. & PRIA: 115r VA: 304r & $\begin{array}{l}\text { BRD: Hallstatt (Knörzer 1971c) P: Hallstatt } \\
\text { (Gluza \& Wasylikowa 1977) }\end{array}$ \\
\hline Veronica sp. & RIA: 308+ GIA: 242r VA: 334+ EMA: 428r & \\
\hline Viburnum opulus $\mathbf{L}$. & VA: $334 \mathrm{r}$ & $\begin{array}{l}\text { BI: VI-VIII (Godwin 1975) P: Early } \\
\text { Medieval (Gluza \& Wasylikowa 1977) }\end{array}$ \\
\hline Vicia cracca L. & VA: $110+$ & $\begin{array}{l}\text { BI: c. } 80-130 \text { A.D. (Helbæk 1964) S: Middle } \\
\text { Iron Age (Helbæk 1955b) }\end{array}$ \\
\hline Vicia cf. cracca $\mathrm{L}$. & RIA: $107+$ VA: $985 \mathrm{r}$ & \\
\hline Vicia faba $L$. & RIA: $311+$ VA: $301 \mathrm{r} 304 \mathrm{c} 307 \mathrm{c} 312 \mathrm{c} 320 \mathrm{c} 334 \mathrm{c}$ & $\begin{array}{l}\text { BI: Iron Age, Roman Period (Godwin } \\
\text { 1975), c. } 80-130 \text { A.D. (Helbæk 1964) NL: } \\
\text { 200 B.C.-250 A.D. (Zeist 1968) BRD: 12-8 } \\
\text { B.C. (Kučan 1981), 1st-2nd century A.D. } \\
\text { (Knörzer 1967c, 1970, 1979b, Körber- } \\
\text { Grohne 1967, 1979b) DDR: Bronze Age } \\
\text { (Jäger 1965), 50 B.C. - 50 A.D. (Lange } \\
\text { 1972), 3rd-4th century A.D. (Schiemann } \\
\text { 1957) P: Hallstatt and later (Gluza \& } \\
\text { Wasylikowa 1977) }\end{array}$ \\
\hline Vicia hirsuta (L.) S. F. Gray & $\begin{array}{l}\text { RIA: 708r VA: } 110+334 \mathrm{c} 985 \mathrm{r} \text { LMA: } 809 \mathrm{r} \\
\text { 957r }\end{array}$ & $\begin{array}{l}\text { BI: 1st-2nd century A.D. (Kenward \& } \\
\text { Williams 1979), Roman Period (Godwin } \\
\text { 1975), c. 80-130 A.D. (Helbæk 1964) BRD: } \\
\text { Band Ceramic (Knörzer 1974a, 1977), 1st } \\
\text { century A.D. (Knörzer 1970) }\end{array}$ \\
\hline $\begin{array}{l}\text { Vicia cf. hirsuta (L.) S. F. } \\
\text { Gray }\end{array}$ & VA: $301+$ & \\
\hline $\begin{array}{l}\text { Vicia hirsuta (L.) S. F. Gray } \\
\text { et V. sativa L. subsp. nigra } \\
\text { (L.) Ehrh. }\end{array}$ & VA: $103 \mathrm{c}$ & \\
\hline Vicia cf. lathyroides L. & VA: $110 \mathrm{r}$ & $\begin{array}{l}\text { Vicia lathyroides recorded from P: Early } \\
\text { Medieval (Gluza \& Wasylikowa 1977) }\end{array}$ \\
\hline $\begin{array}{l}\text { Vicia orobus DC. in Lam. \& } \\
\text { DC. }\end{array}$ & VA: $110 \mathrm{r}$ & \\
\hline $\begin{array}{l}\text { Vicia sativa L. subsp. nigra } \\
\text { (L.) Ehrh. (V. angustifolia } \\
\text { L.) }\end{array}$ & VA: 110 r 334+ EMA: $992+$ LMA: 809 r & $\begin{array}{l}\text { BI: c. 80-130 A.D. (Helbak 1964) NL: } 1000 \\
\text { B.C. (Pals 1977), 9th-12th century A.D. } \\
\text { (Zeist \& Palfenier-Vegter 1979) BRD: } \\
\text { Hallstatt (Knörzer 1971c), c. 200 B.C. } \\
\text { (Knörzer 1979a), 1st century A.D. (Knörzer } \\
\text { 1970) DDR: 3rd century A.D. (Lange 1973) }\end{array}$ \\
\hline
\end{tabular}




\begin{tabular}{|c|c|c|}
\hline Species & $\begin{array}{l}\text { Period, site number (cf. Table 1) and number } \\
\text { of macrofossils reported }\end{array}$ & $\begin{array}{l}\text { Reports on finds from neighbour countries. } \\
\text { Pollen zones and other periods are presented } \\
\text { as published by the authors cited }\end{array}$ \\
\hline $\begin{array}{l}\text { Vicia cf. sativa L. subsp. } \\
\text { nigra (L.) Ehrh. (V. } \\
\text { angustifolia L.) }\end{array}$ & PRIA: 301r GIA: 301r VA: 202r 301+ & \\
\hline $\begin{array}{l}\text { Vicia tetrasperma (L.) } \\
\text { Schreber }\end{array}$ & RIA: $708 \mathrm{r}$ VA: $110+304 \mathrm{c} 312 \mathrm{r} 334 \mathrm{c}$ & $\begin{array}{l}\text { BI: Early Iron Age (Helbæk 1952), Iron } \\
\text { Age, Roman Period (Godwin 1975), 1st- } \\
\text { early 2nd century A.D. (Kenward \& } \\
\text { Williams 1979), c. 80-130 A.D. (Helbæk } \\
\text { 1964) BRD: Band Ceramic (Knörzer 1974a), } \\
\text { 1st century A.D. (Knörzer 1970), 150-260 } \\
\text { A.D. (Körber-Grohne \& Piening 1979), 3rd } \\
\text { century A.D. (Lange 1973) }\end{array}$ \\
\hline $\begin{array}{l}\text { Vicia cf. tetrasperma (L.) } \\
\text { Schreber }\end{array}$ & PRIA: 301r VA: 301r & \\
\hline Vicia villosa Roth & VA: $985 r$ & \\
\hline Vicia sp. & $\begin{array}{l}\text { PRIA: } 301+\text { RIA: } 301+308+\text { GIA: } 301+ \\
\text { VA: } 301+334 c\end{array}$ & \\
\hline Viola arvensis Murray & PRIA: $111 \mathrm{r} 114+115+$ GIA: $712 \mathrm{r}$ LMA: $504 \mathrm{r}$ & NL: 1000 B.C. (Pals 1977) \\
\hline Viola cf. arvensis Murray & LMA: $406 \mathrm{r}$ & \\
\hline $\begin{array}{l}\text { Viola arvensis Murray, V. } \\
\text { canina } L \text {. et V. tricolor L. }\end{array}$ & VA: $202+$ & \\
\hline Viola canina $\mathrm{L}$. & VIII: $220 \mathrm{c}$ GIA: $135+$ & BI: I/II-II, VIIb (Godwin 1975) \\
\hline Viola cf. canina $\mathbf{L}$. & VIII: $207+219+230 \mathrm{r}$ & \\
\hline Viola palustris $\mathbf{L}$. & III: $161+u$ V: 104 r RIA: $101+301+$ & BI: I-VIII (Godwin 1975) \\
\hline Viola cf. palustris L. & IX: 328r 501r 502+ & \\
\hline $\begin{array}{l}\text { Viola reichenbachiana } \\
\text { Jordan ex Boreau (V. } \\
\text { sylvestris Lam. pro parte) }\end{array}$ & VA: $334 c$ & \\
\hline Viola tricolor $\mathrm{L}$. & VIII: $220 \mathrm{c}$ RIA: $311 \mathrm{r}$ VA: $304 \mathrm{c} 334 \mathrm{c}$ & \\
\hline Viola sp. & $\begin{array}{l}\text { IX: } 513 \text { r RIA: } 301 \mathrm{r} \text { GIA: } 242 \mathrm{r} 244+301 \mathrm{r} \\
\text { VA: } 172 \mathrm{r} \text { 301r } 304+334+\text { EMA: } 172+428 \mathrm{r} \\
\text { 429+ 521cu LMA: } 241+427+429+521+\mathrm{u} \\
522 \mathrm{r}\end{array}$ & \\
\hline Viscum album $\mathrm{L}$. & VI, VII: $179 \mathrm{r}$ 560ru & BI: VI-VIII (Godwin 1975) \\
\hline Vitis vinifera $L$. & VA: 312r 334r LMA: 521ru & $\begin{array}{l}\text { BI: Roman Period (Godwin 1975), 1st-4th } \\
\text { century A.D. (Willcox 1977), 4th-5th } \\
\text { century A.D. (Greig 1976) BRD: 12-8 B.C. } \\
\text { (Kucan 1981), 1st-2nd century A.D. } \\
\text { (Knörzer 1967b, 1973) P: 9th-10th century } \\
\text { A.D. (Wasylikowa 1978) }\end{array}$ \\
\hline Xanthium strumarium L. & EMA: 521ru & $\begin{array}{l}\text { DDR: Migration Period, 6th-10th century } \\
\text { A.D. (Lange 1976, 1979) P: 11th-12th } \\
\text { century A.D. (Wasylikowa 1978), Early } \\
\text { Medieval (Gluza \& Wasylikowa 1977) }\end{array}$ \\
\hline Zannichellia palustris $\mathrm{L}$. & $\begin{array}{l}\text { III: } 104+\text { VI, VII: 520cu 528ru 553+u VIII: } \\
\text { 822c } 826+\text { GIA: 553r VA: 304r } 307+334+ \\
\text { EMA: } 431+521 \mathrm{cu} \text { LMA: } 430 \mathrm{c} 521 \mathrm{ru}\end{array}$ & BI: II-IV, VIIb-VIII (Godwin 1975) \\
\hline Zostera marina $\mathrm{L}$. & $\begin{array}{l}\text { VI, VII: } 520 \mathrm{cu} 818+826+\text { VIII: } 818+826+ \\
\text { EMA: } 521+\mathrm{u}\end{array}$ & BI: VIIa (Godwin 1975) \\
\hline $\begin{array}{l}\text { Zostera noltii Hornem. } \\
\text { (Z. nana Roth pro parte) }\end{array}$ & GIA: $243+$ EMA: $431+$ & \\
\hline
\end{tabular}


Table 3.

Finds of macrofossils from Denmark, Schleswig, Scania, Halland, and Blekinge referred to periods between 13,000 B.P. and 1536 A.D.

$\times$ : find(s) recorded, $u$ : find(s) recorded - dating uncertain.

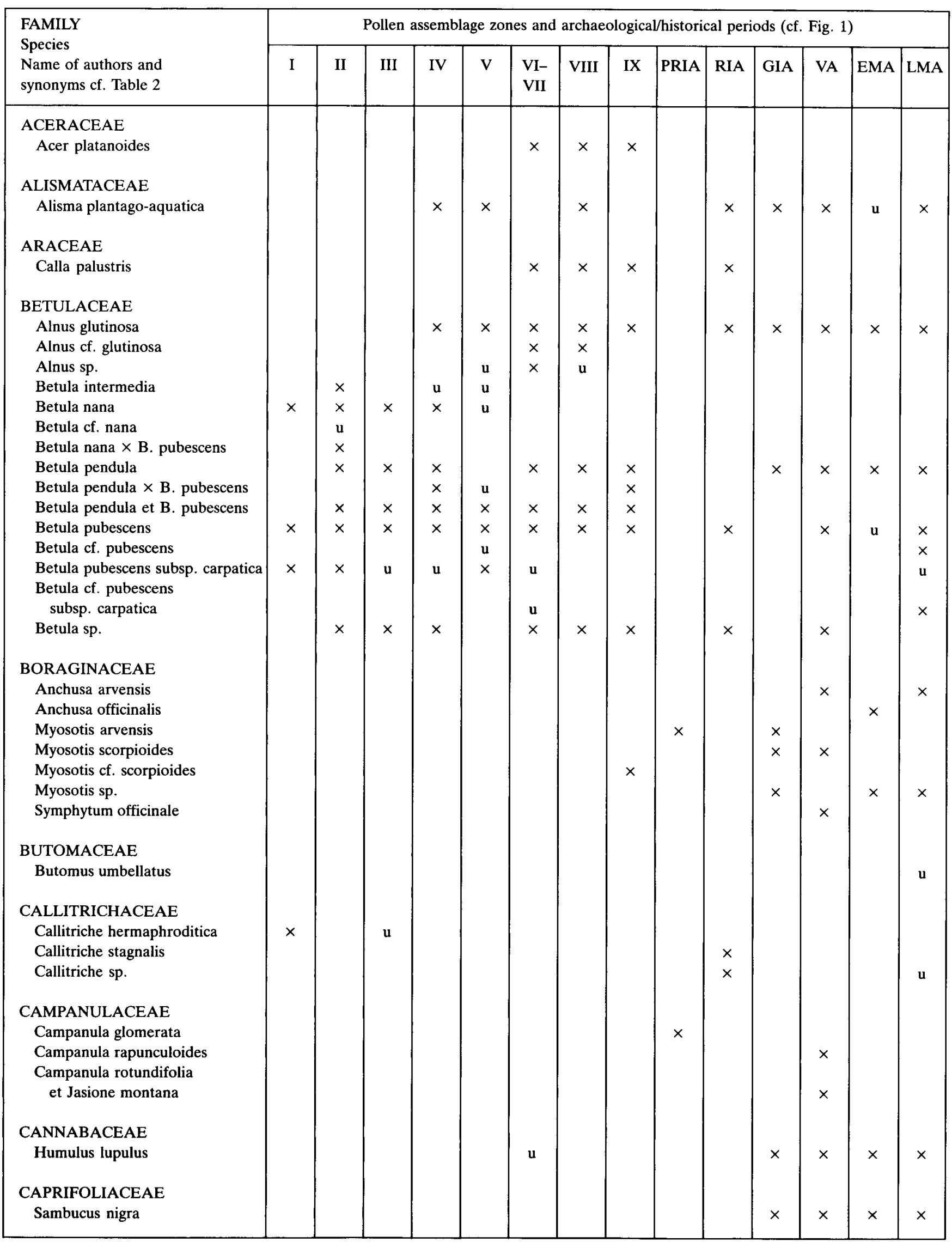




\begin{tabular}{|c|c|c|c|c|c|c|c|c|c|c|c|c|c|c|}
\hline \multirow{2}{*}{$\begin{array}{l}\text { FAMILY } \\
\text { Species } \\
\text { Name of authors and } \\
\text { synonyms cf. Table } 2\end{array}$} & \multicolumn{14}{|c|}{ Pollen assemblage zones and archaeological/historical periods (cf. Fig. 1) } \\
\hline & I & II & III & IV & $\mathbf{V}$ & $\begin{array}{l}\text { VI- } \\
\text { VII }\end{array}$ & VIII & IX & PRIA & RIA & GIA & VA & EMA & LMA \\
\hline $\begin{array}{l}\text { Sambucus cf. nigra } \\
\text { Viburnum opulus }\end{array}$ & & & & & & & & & & & & $x$ & $x$ & $\times$ \\
\hline \multicolumn{15}{|l|}{ CARYOPHYLLACEAE } \\
\hline Agrostemma githago & & & & & & & & & & $x$ & $x$ & $x$ & $x$ & $x$ \\
\hline Arenaria serpyllifolia & & & & & & & $x$ & & & & & $\times$ & $x$ & $\mathbf{u}$ \\
\hline $\begin{array}{l}\text { Cerastium fontanum } \\
\text { subsp. triviale }\end{array}$ & & & & & & & & & $x$ & $x$ & $x$ & $x$ & & $x$ \\
\hline Cerastium sp. & & & & & & & & & & $x$ & & $x$ & $x$ & $\mathbf{u}$ \\
\hline Dianthus deltoides & & & & & & & & & & & & & $x$ & \\
\hline Lychnis flos-cuculi & & & & & & $x$ & $\times$ & $\times$ & & $x$ & $\times$ & $\times$ & $x$ & $\times$ \\
\hline Moehringia trinervia & & & & & & $\mathbf{u}$ & & & & $x$ & & & & $\times$ \\
\hline Myosoton aquaticum & & & & & & & & & & $\times$ & & & & \\
\hline Sagina cf. procumbens & & & & & & & & & & $\times$ & & & & \\
\hline Sagina sp. & & & & & & & & & & $x$ & & $\times$ & & \\
\hline Saponaria officinalis & & & & & & & & & & & & $\times$ & & \\
\hline Scleranthus annuus & & & & & & & $x$ & & $x$ & $\times$ & & $x$ & $x$ & $x$ \\
\hline Scleranthus cf. annuus & & & & & & & & & & $x$ & & & & \\
\hline Scleranthus sp. & & & & & & & & & $x$ & $x$ & $x$ & & $x$ & \\
\hline Silene alba & & & & & & & $x$ & & $x$ & $x$ & $x$ & $x$ & $\mathrm{u}$ & $x$ \\
\hline Silene dioica & & & & & & & & & & & & $x$ & & \\
\hline Silene noctiflora & & & & & & & $x$ & & & $x$ & & $x$ & & \\
\hline Silene vulgaris & & & & & & & & & & & & $x$ & & $x$ \\
\hline Silene cf. vulgaris & & & & & & & & & & & & & $x$ & \\
\hline Silene sp. & & & & & & & & & & & $\times$ & $x$ & $x$ & \\
\hline Spergula arvensis & & & & & & & $x$ & & $x$ & $x$ & $x$ & $x$ & $x$ & $x$ \\
\hline Spergula sp. & & & & & & & $x$ & & & & & & $x$ & \\
\hline Spergularia marina & & & & & & & & & & $x$ & & $x$ & & \\
\hline Spergularia cf. marina & & & & & & & & & & $x$ & & & & \\
\hline Spergularia media & & & & & & & & & & $x$ & & $x$ & & \\
\hline Stellaria alsine & & & & & & & & & & $x$ & & & $\mathbf{u}$ & \\
\hline Stellaria graminea & & $x$ & & & & & $x$ & & $x$ & & & $x$ & $x$ & $x$ \\
\hline Stellaria cf. graminea & & & & & & & $x$ & & & & & & & \\
\hline Stellaria media & & & & & & & $x$ & & $x$ & $x$ & $x$ & $x$ & $x$ & $x$ \\
\hline Stellaria cf. nemorum & & & & & & & & & & & & $x$ & & \\
\hline Stellaria palustris & & & & & & & & & & $x$ & $x$ & $x$ & & \\
\hline Stellaria cf. palustris & & & & & & & & & $x$ & $x$ & $x$ & $\times$ & & \\
\hline Stellaria sp. & & & & & & & & $x$ & & & & & & \\
\hline CERATOPHYLLACEAE & & & & & & & & & & & & & & \\
\hline Ceratophyllum demersum & & & $x$ & $x$ & $\mathbf{u}$ & $x$ & $x$ & & & & & & $x$ & $x$ \\
\hline Ceratophyllum submersum & & & & & $x$ & $\mathbf{u}$ & & & & & & & r & $\mathbf{u}$ \\
\hline Ceratophyllum sp. & & & & & $x$ & $x$ & $x$ & $x$ & & & & & & \\
\hline CHENOPODIACEAE & & & & & & & & & & & & & & \\
\hline Atriplex hastata & & & & & & & & & & $x$ & $x$ & $x$ & & \\
\hline Atriplex cf. hastata & & & & & & & & & $x$ & $x$ & $x$ & $\times$ & & \\
\hline Atriplex hastata et A. littoralis & & & & & & & & & & $x$ & & $x$ & & \\
\hline $\begin{array}{l}\text { Atriplex hastata, A. littoralis } \\
\text { et A. patula }\end{array}$ & & & & & & & & & & & & $x$ & & \\
\hline Atriplex littoralis & & & & & & $\mathbf{u}$ & & & & & & & & \\
\hline Atriplex patula & & & & & & $\mathbf{u}$ & & & & $x$ & $\times$ & $x$ & & \\
\hline Atriplex cf. patula & & & & & & & & & $x$ & $x$ & $x$ & $x$ & & \\
\hline Atriplex sp. & & & & & $x$ & $x$ & & & $x$ & $x$ & $x$ & $x$ & $x$ & $x$ \\
\hline Chenopodium album & & & & & & $x$ & $x$ & $x$ & $x$ & $x$ & $x$ & $x$ & $x$ & $x$ \\
\hline Chenopodium cf. album & & & & & & & & & & $x$ & $x$ & $x$ & $x$ & $x$ \\
\hline Chenopodium ficifolium & & & & & & & & & & $x$ & & $x$ & & \\
\hline Chenopodium cf. glaucum & & & & & & & $x$ & $x$ & & & & & & \\
\hline $\begin{array}{l}\text { Chenopodium glaucum et } \\
\text { C. rubrum }\end{array}$ & & & & & & & & & $x$ & $x$ & & & & \\
\hline
\end{tabular}




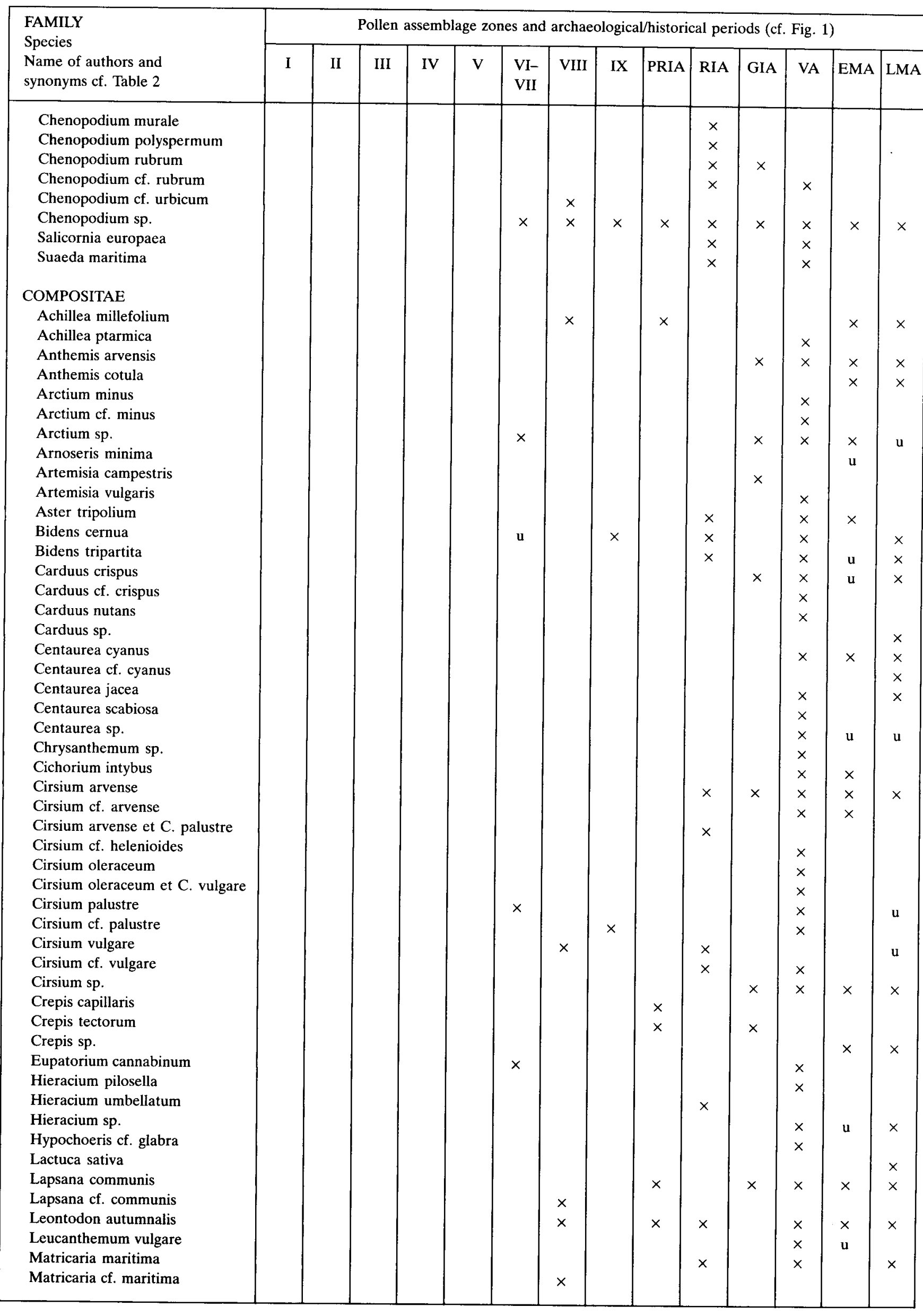




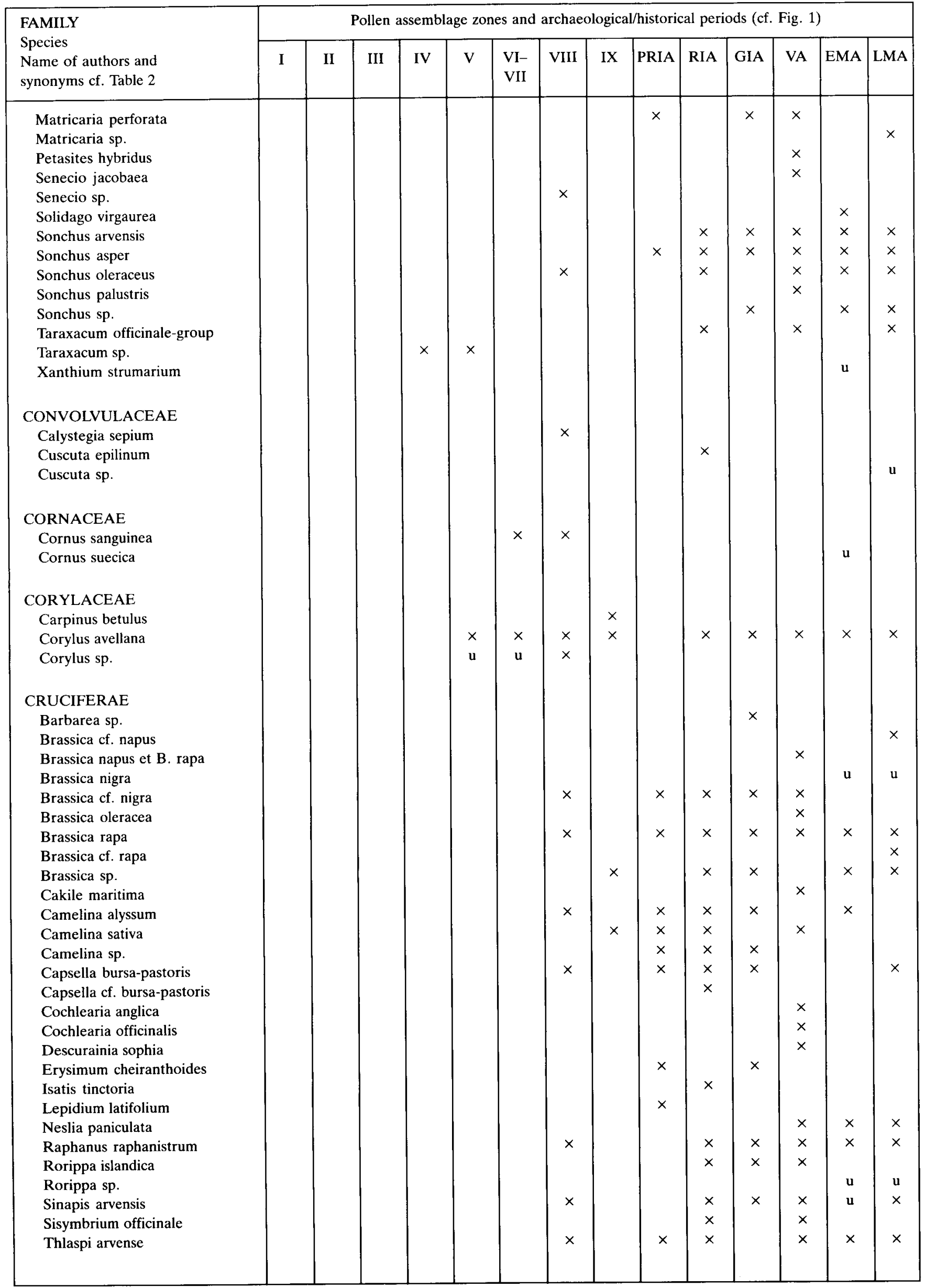




\begin{tabular}{|c|c|c|c|c|c|c|c|c|c|c|c|c|c|c|}
\hline \multirow{2}{*}{$\begin{array}{l}\text { FAMILY } \\
\text { Species } \\
\text { Name of authors and } \\
\text { synonyms cf. Table } 2\end{array}$} & \multicolumn{14}{|c|}{ Pollen assemblage zones and archaeological/historical periods (cf. Fig. 1) } \\
\hline & I & II & III & IV & $\mathrm{V}$ & $\begin{array}{l}\text { VI- } \\
\text { VII }\end{array}$ & VIII & IX & PRIA & RIA & GIA & VA & EMA & LMA \\
\hline \multicolumn{15}{|l|}{$\begin{array}{l}\text { CUPRESSACEAE } \\
\text { Juniperus communis }\end{array}$} \\
\hline \multicolumn{15}{|l|}{ CYPERACEAE } \\
\hline Blysmus compressus & & & & & & & & & & & & & $\mathbf{u}$ & $\mathbf{u}$ \\
\hline Blysmus rufus & & & & & & & & & & & & & u & \\
\hline Carex acuta & & & & & & & & $\mathbf{u}$ & & & $x$ & $x$ & & \\
\hline Carex cf. acuta & & & & & & & $\mathbf{u}$ & $\times$ & & & & & & \\
\hline Carex appropinquata & & & & & & & & & & & & $x$ & & \\
\hline Carex cf. appropinquata & & & & & & & & & & $x$ & & & & \\
\hline Carex cf. aquatilis & & & $x$ & & & & & & & & & & & \\
\hline Carex arenaria & & & & & & & & & & $x$ & & & & \\
\hline Carex cespitosa & & & & & & & & $x$ & & & & & & \\
\hline Carex curta & & & & & & $x$ & $x$ & $x$ & & & & & & $\times$ \\
\hline Carex cf. curta & & & & & & & & $x$ & $x$ & $x$ & $x$ & $x$ & & \\
\hline Carex diandra & & & & & & & & $x$ & & & & & & $x$ \\
\hline Carex cf. diandra & & & & & & & $\mathbf{u}$ & & & & & & & \\
\hline Carex distans & & & & & & & & & & & & $x$ & & \\
\hline Carex cf. distans & & & & & & & & & & $x$ & $x$ & $x$ & & \\
\hline Carex disticha & & & & & & & & & $x$ & $x$ & $x$ & $x$ & & $x$ \\
\hline Carex disticha et C. elongata & & & & & & & & & & & & $\times$ & & \\
\hline $\begin{array}{l}\text { Carex sect. Distigmaticae } \\
\text { Carex echinata }\end{array}$ & $x$ & $x$ & $x$ & & & & $x$ & & & & $x$ & $x$ & $x$ & $x$ \\
\hline Carex cf. echinata & & & & & & & $x$ & & & & & $x$ & $x$ & $\times$ \\
\hline Carex elata & & & & & & & $x$ & & & & & $x$ & & \\
\hline Carex elongata & & & & & & & & $x$ & & & & & & \\
\hline Carex cf. elongata & & & & & & & & $x$ & & & & & & \\
\hline Carex extensa & & & & & & & & & & & & $x$ & & \\
\hline Carex flacca & & & & & & & & & & & & $x$ & & $\times$ \\
\hline Carex flava & & & & & & & & & & $x$ & & $x$ & & \\
\hline Carex cf. flava & & & & & & & $x$ & & $x$ & $x$ & $x$ & $x$ & & \\
\hline Carex hirta & & & & & & & & & $x$ & $x$ & $x$ & $x$ & $\mathrm{u}$ & $\mathbf{u}$ \\
\hline Carex lasiocarpa & & & & $x$ & $x$ & $x$ & $x$ & $x$ & & & & & & $x$ \\
\hline Carex lepidocarpa & & & & & & & & & & & $\times$ & & & $\hat{n}$ \\
\hline Carex nigra & & & & & & & & & & & & $x$ & & $x$ \\
\hline Carex cf. nigra & & & & & & & & & $x$ & $x$ & $x$ & $x$ & & \\
\hline Carex nigra et $\mathrm{C}$. rostrata & & & & & & $x$ & $x$ & $x$ & & & & & & \\
\hline Carex ovalis & & & & & & & & & $x$ & & & & $\mathrm{u}$ & \\
\hline Carex panicea & & & & & & & & & & & & $\times$ & & \\
\hline Carex cf. panicea & & & & & & & & & & & & $x$ & & \\
\hline Carex cf. paniculata & & & & & & & & $x$ & & & & & & \\
\hline Carex pilulifera & & & & & & & $x$ & & & $x$ & & $x$ & & \\
\hline Carex pseudocyperus & & & & $x$ & $\mathbf{u}$ & $x$ & $x$ & $x$ & & $x$ & $x$ & $x$ & & $\mathbf{u}$ \\
\hline Carex riparia & & & & & $\mathbf{u}$ & & & & & & & $x$ & & \\
\hline Carex cf. riparia & & & & & & & & & $x$ & $x$ & $x$ & $\times$ & & \\
\hline Carex rostrata & & $x$ & $x$ & $x$ & u & $x$ & & $x$ & & $x$ & $x$ & $x$ & & $x$ \\
\hline Carex cf. rostrata & $x$ & $x$ & $x$ & & & & $x$ & & & & & & & \\
\hline Carex cf. spicata & & & & & & & & & & & & $x$ & & \\
\hline Carex strigosa & & & & & & & & & & & & $x$ & & \\
\hline Carex, sect. Tristigmaticae & & $x$ & $x$ & & & & & & & & $x$ & $x$ & $x$ & $\times$ \\
\hline Carex vesicaria & & $x$ & & $x$ & & & $\mathbf{u}$ & $x$ & & $x$ & & & $\mathrm{u}$ & $x$ \\
\hline Carex cf. vesicaria & & & & $x$ & & & & & & & & & & \\
\hline Carex vulpina & & & & & & & & & & $x$ & & $x$ & & \\
\hline Carex cf. vulpina & & & & & & & & & $x$ & $x$ & $x$ & $x$ & & \\
\hline Carex sp. & $x$ & $x$ & $x$ & $x$ & $x$ & $x$ & $x$ & $x$ & $x$ & $x$ & $x$ & $x$ & $x$ & $x$ \\
\hline Cladium mariscus & & & & & $\times$ & $x$ & $x$ & $x$ & & $x$ & & & & $\mathbf{u}$ \\
\hline Cyperus fuscus & & & & & & & & & & & & & $\mathrm{u}$ & . \\
\hline Eleocharis palustris & & & $\mathbf{u}$ & & & $\mathbf{u}$ & $x$ & $x$ & $x$ & $x$ & $x$ & $x$ & $x$ & $x$ \\
\hline Eleocharis cf. palustris & & & & & & & & $x$ & & $x$ & & & - & - \\
\hline Eleocharis palustris et E. uniglumis & & & & & & & & & & & $x$ & $x$ & $x$ & $x$ \\
\hline
\end{tabular}




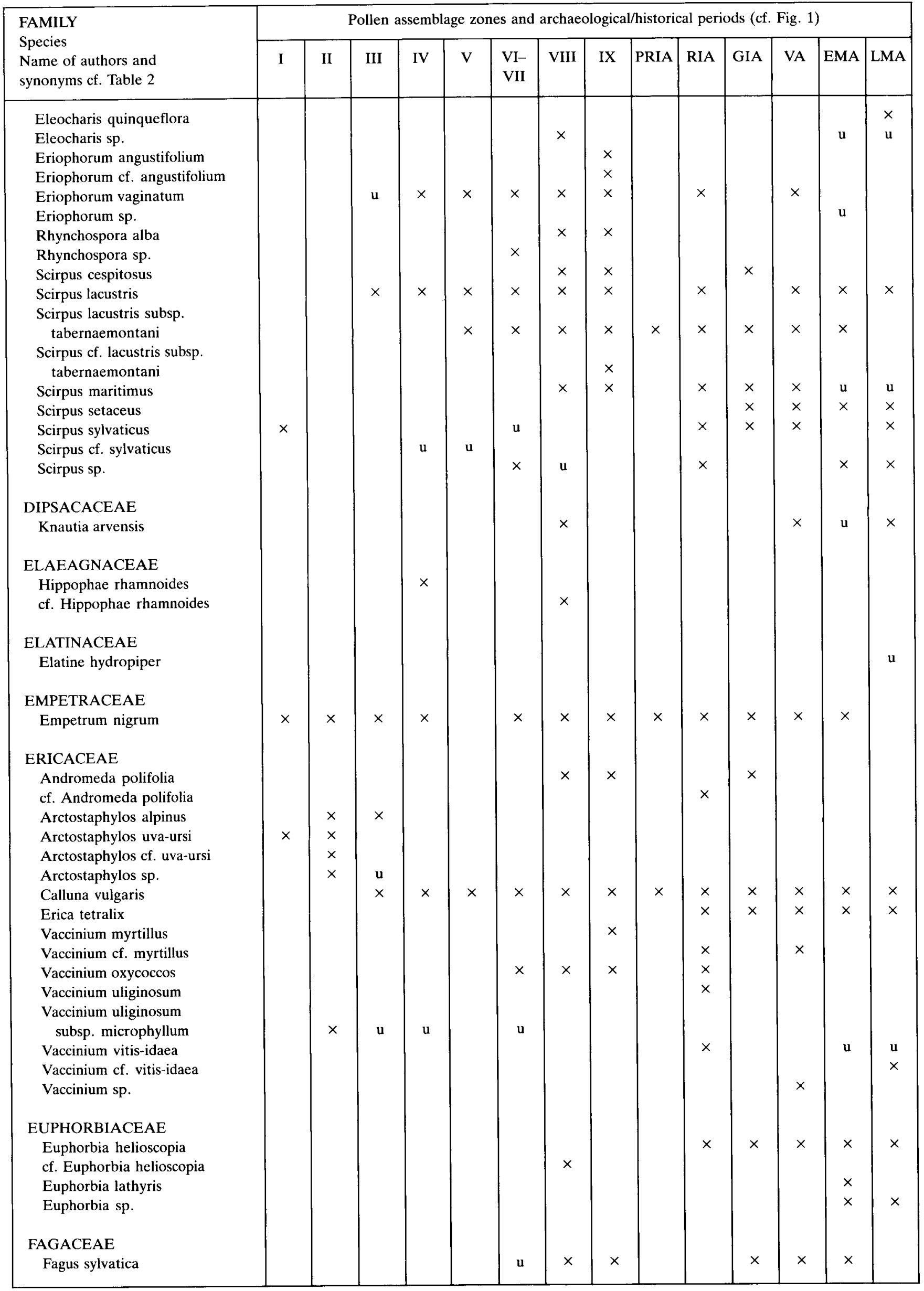

(continued) 


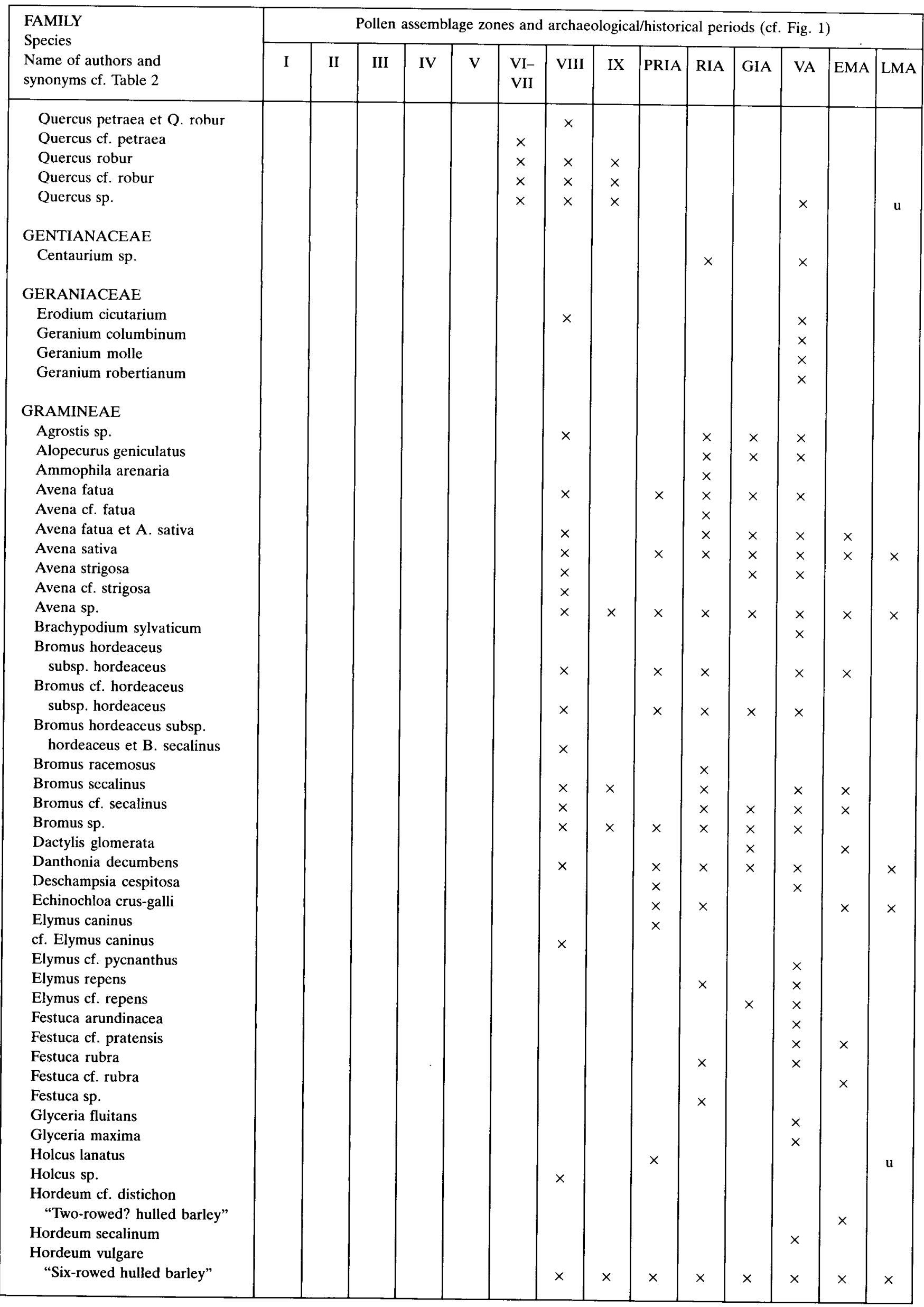




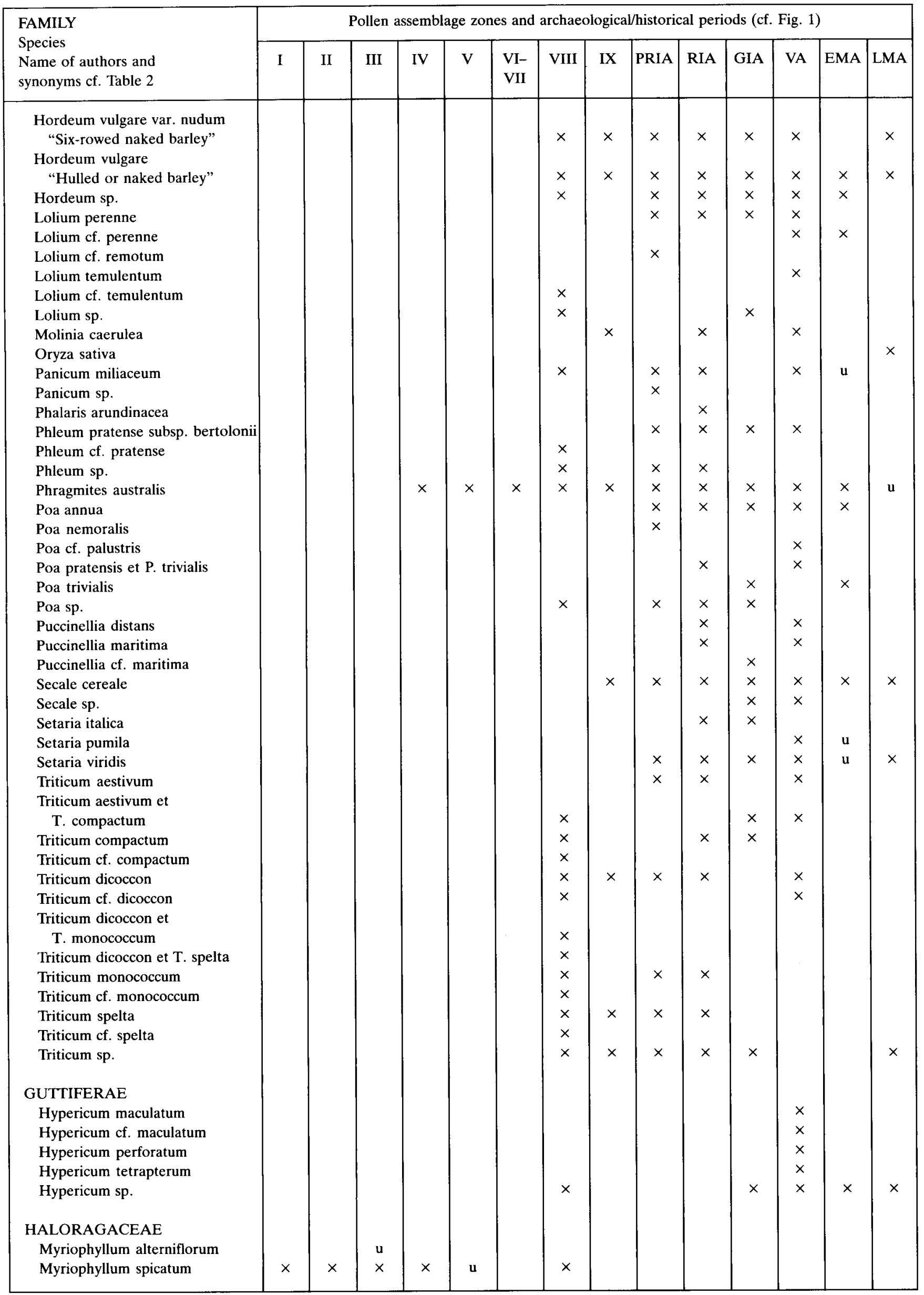




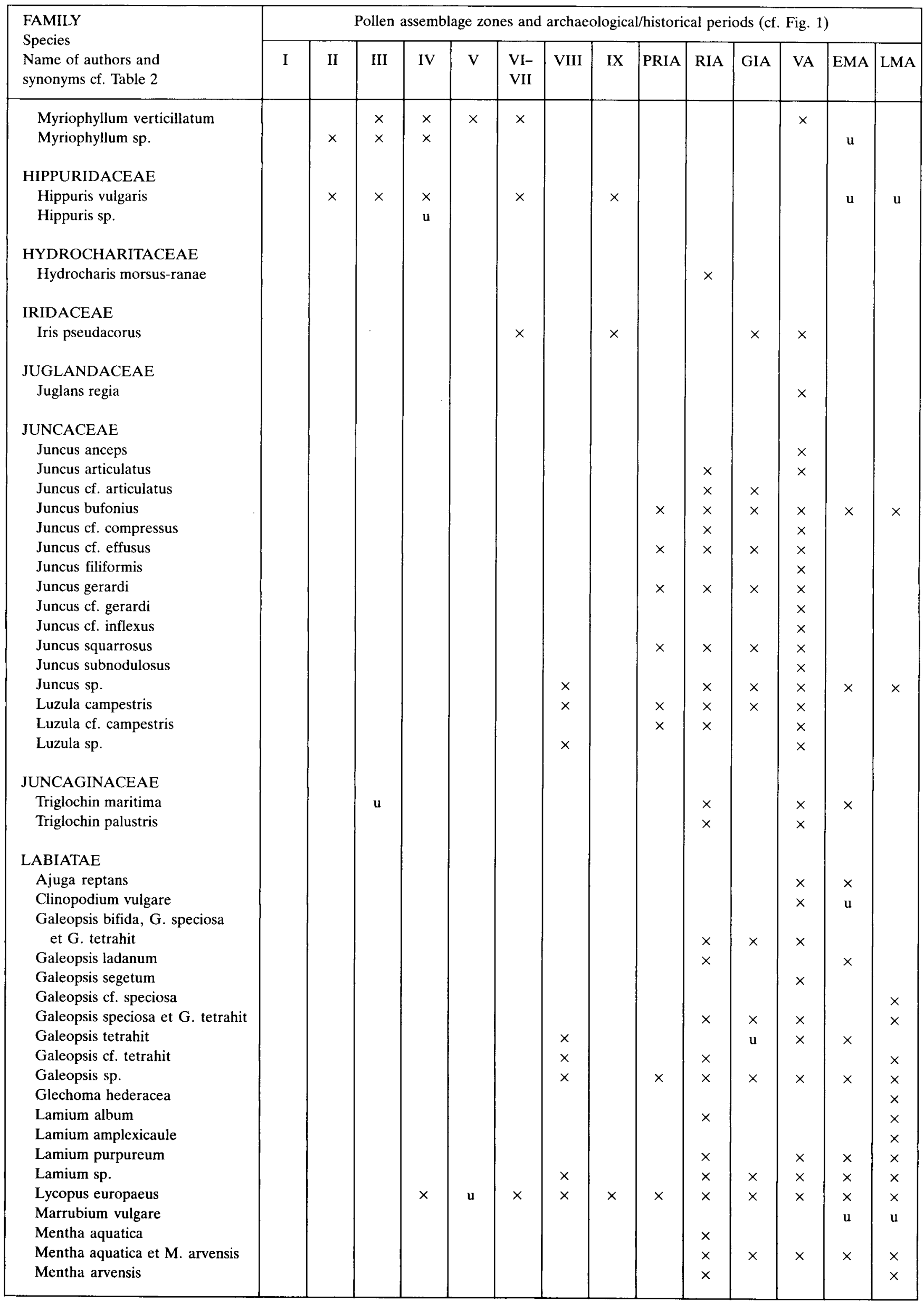




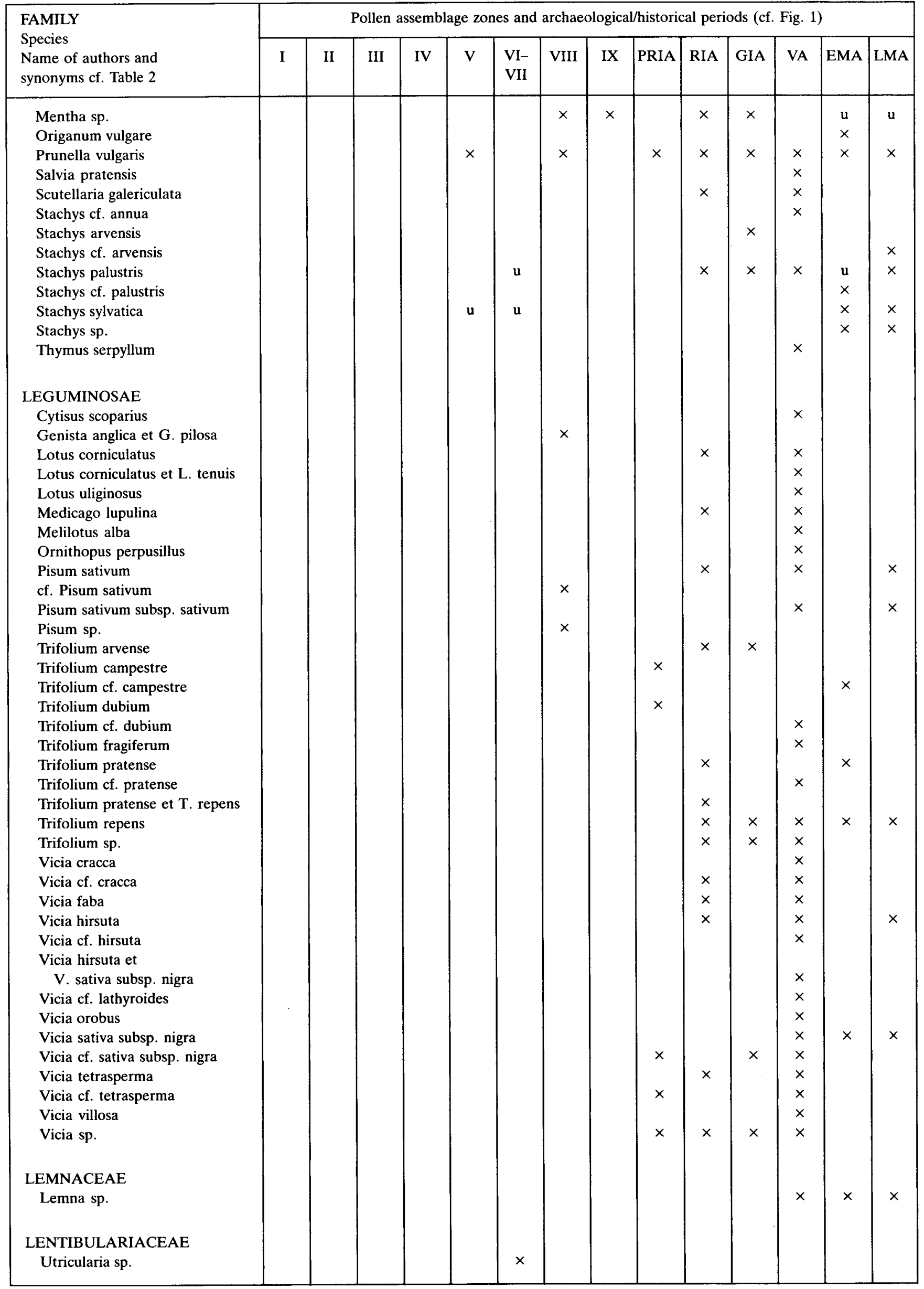




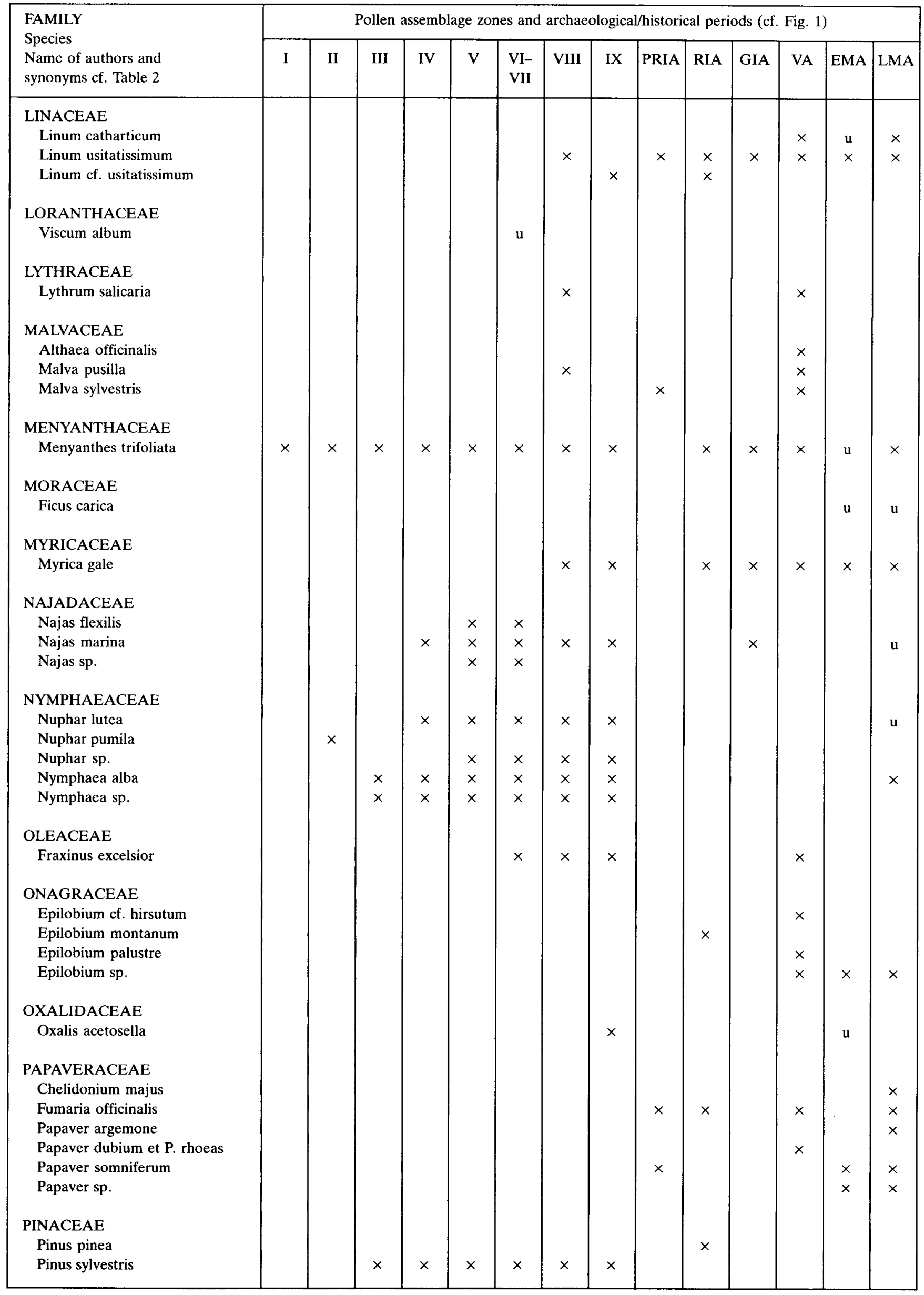




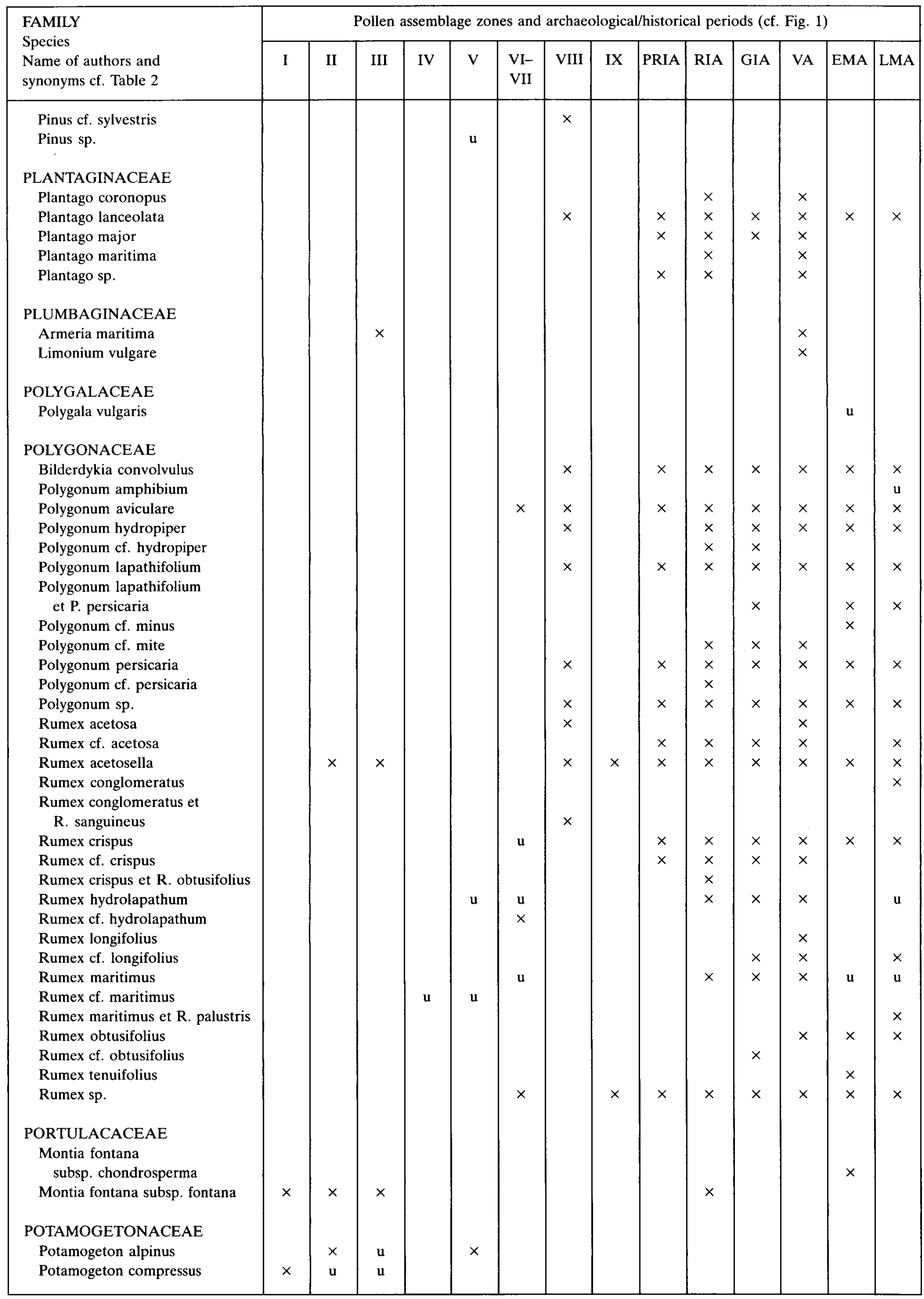




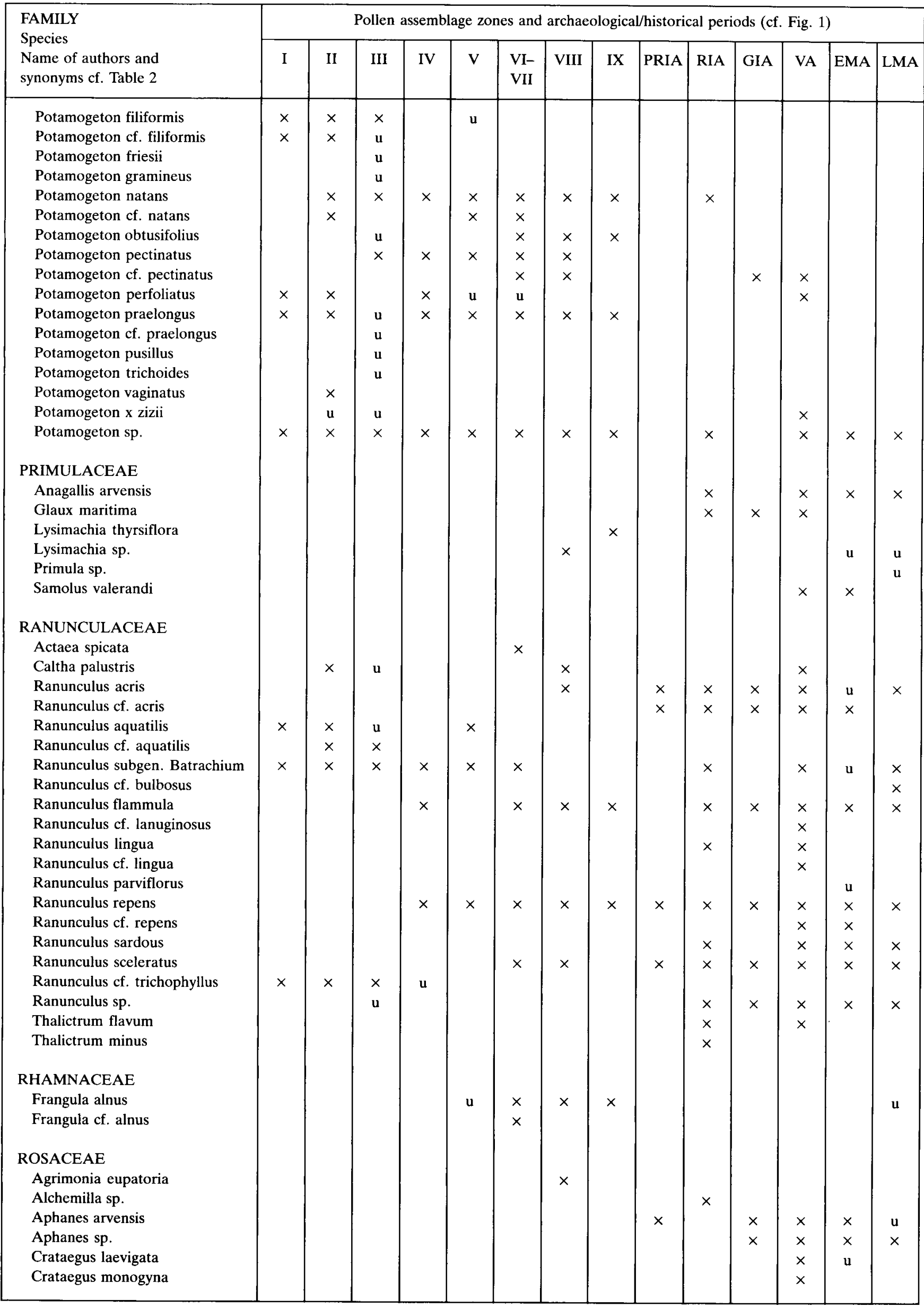




\begin{tabular}{|c|c|c|c|c|c|c|c|c|c|c|c|c|c|c|}
\hline \multirow{2}{*}{$\begin{array}{l}\text { FAMILY } \\
\text { Species } \\
\text { Name of authors and } \\
\text { synonyms cf. Table } 2\end{array}$} & \multicolumn{14}{|c|}{ Pollen assemblage zones and archaeological/historical periods (cf. Fig. 1) } \\
\hline & $\mathbf{I}$ & II & III & IV & $\mathrm{V}$ & $\begin{array}{l}\text { VI- } \\
\text { VII }\end{array}$ & VIII & IX & PRIA & RIA & GIA & VA & EMA & LMA \\
\hline Crataegus sp. & & & & & & $\mathbf{u}$ & $x$ & & & & & & $x$ & \\
\hline Dryas octopetala & $x$ & $x$ & $\times$ & & u & & & & & & & & & \\
\hline Filipendula ulmaria & & & & & & & u & & & & & $\times$ & & $x$ \\
\hline Fragaria vesca & & & & & & & $x$ & & & & $x$ & $x$ & $x$ & $x$ \\
\hline Fragaria sp. & & & & & & & & & & & & & $\mathbf{u}$ & u \\
\hline Geum sp. & & & & & u & $\mathbf{u}$ & & & & & & & & \\
\hline Malus sylvestris & & & & & & & $x$ & & & & & $x$ & $x$ & $x$ \\
\hline Malus sp. & & & & & & & $\times$ & & & & & & & \\
\hline Potentilla anserina & & & & & $x$ & & & $x$ & & $x$ & $x$ & $x$ & $x$ & $x$ \\
\hline Potentilla argentea & & & & & & & & & $x$ & & & $x$ & $x$ & $x$ \\
\hline Potentilla cf. argentea & & & & & & & & & & & & & $x$ & \\
\hline Potentilla erecta & & & $\mathbf{u}$ & & & & $x$ & $x$ & $x$ & $x$ & $x$ & $x$ & $x$ & $x$ \\
\hline Potentilla palustris & $x$ & $x$ & $x$ & $x$ & $x$ & $x$ & $x$ & $x$ & & $x$ & $x$ & $x$ & $\times$ & $x$ \\
\hline cf. Potentilla palustris & & & & & & & & & & & & & $x$ & \\
\hline Potentilla reptans & & & & & & & & & & & $x$ & & & $x$ \\
\hline Potentilla cf. reptans & & & & & & & & & & & & $x$ & & \\
\hline Potentilla sp. & & $x$ & u & & & & $x$ & & $x$ & $x$ & $x$ & $x$ & $x$ & $x$ \\
\hline Prunus avium & & & & & & & & & & & & $x$ & & $x$ \\
\hline cf. Prunus avium & & & & & & & & & & & & & & $x$ \\
\hline Prunus cerasus & & & & & & & & & & & & & & $x$ \\
\hline Prunus subgen. Cerasus & & & & & & & & & & & & & $\mathbf{u}$ & $\mathbf{u}$ \\
\hline Prunus domestica & & & & & & & & & & & & & $\times$ & $x$ \\
\hline Prunus domestica subsp. insititia & & & & & & & & & & & & $x$ & $x$ & $x$ \\
\hline $\begin{array}{l}\text { Prunus cf. domestica } \\
\text { subsp. insititia }\end{array}$ & & & & & & & & & & & & & $x$ & \\
\hline Prunus padus & & & & $x$ & & u & & & & & & & & \\
\hline Prunus persica & & & & & & & & & & & & $x$ & & \\
\hline Prunus spinosa & & & & & & & $\times$ & & & & & $x$ & $\mathbf{u}$ & $x$ \\
\hline Pyrus communis & & & & & & & & & & & & & & $x$ \\
\hline Pyrus sp. & & & & & & & & & & & & & $\mathbf{u}$ & $\mathbf{u}$ \\
\hline Rosa sp. & & & & & & & & & & & & $x$ & $x$ & $x$ \\
\hline Rubus caesius & & & & & & & & & & & & & & $x$ \\
\hline Rubus cf. caesius & & & & & & & $x$ & & & & & & & \\
\hline Rubus corylifolius & & & & & & & & & & & & & $x$ & \\
\hline Rubus fruticosus-group & & & & & & & $x$ & & & $x$ & u & $x$ & $x$ & $x$ \\
\hline Rubus cf. fruticosus & & & & & & & & & & & $x$ & & & \\
\hline Rubus idaeus & & & u & & $\mathbf{u}$ & $x$ & $x$ & $x$ & & $x$ & & $x$ & $x$ & $x$ \\
\hline Rubus saxatilis & & $x$ & & & $\mathbf{u}$ & & & & & & & & & \\
\hline Rubus sp. & & & & & & & & & & & $x$ & & $x$ & $\times$ \\
\hline Sorbus aucuparia & & & & & & $\mathbf{u}$ & $\mathbf{u}$ & $\times$ & & & & $x$ & & \\
\hline Sorbus cf. intermedia & & & & & & & $x$ & & & & & & & \\
\hline RUBIACEAE & & & & & & & & & & & & & & \\
\hline Galium aparine & & & & & & & $x$ & $x$ & $x$ & $x$ & $x$ & $x$ & & $x$ \\
\hline cf. Galium aparine & & & & & & & $x$ & & & & & & & \\
\hline Galium mollugo & & & & & & & & & & $x$ & & $x$ & & \\
\hline Galium cf. mollugo & & & & & & & & & $x$ & & & $x$ & & \\
\hline Galium palustre & & & & & & & & & & $x$ & & $x$ & & \\
\hline Galium cf. palustre & & & & & & & & & $x$ & $x$ & $x$ & $x$ & & \\
\hline Galium spurium & & & & & & & & & & $x$ & $x$ & $x$ & & $x$ \\
\hline Galium sp. & & & & & & & $x$ & & & & & $x$ & $x$ & $x$ \\
\hline RUPPIACEAE & & & & & & & & & & & & & & \\
\hline Ruppia cirrhosa & & & & & & $\mathbf{u}$ & & & & & & & & \\
\hline Ruppia maritima & & & & & $x$ & & $x$ & & & & & $x$ & & \\
\hline Ruppia sp. & & & & & & $x$ & $x$ & $x$ & & & & & $\mathbf{u}$ & $\mathbf{u}$ \\
\hline SALICACEAE & & & & & & & & & & & & & & \\
\hline Populus tremula & & & & $x$ & $x$ & $x$ & $x$ & & & & & $x$ & & \\
\hline Salix cf. arbuscula & & $x$ & & & & & & & & & & & & \\
\hline
\end{tabular}




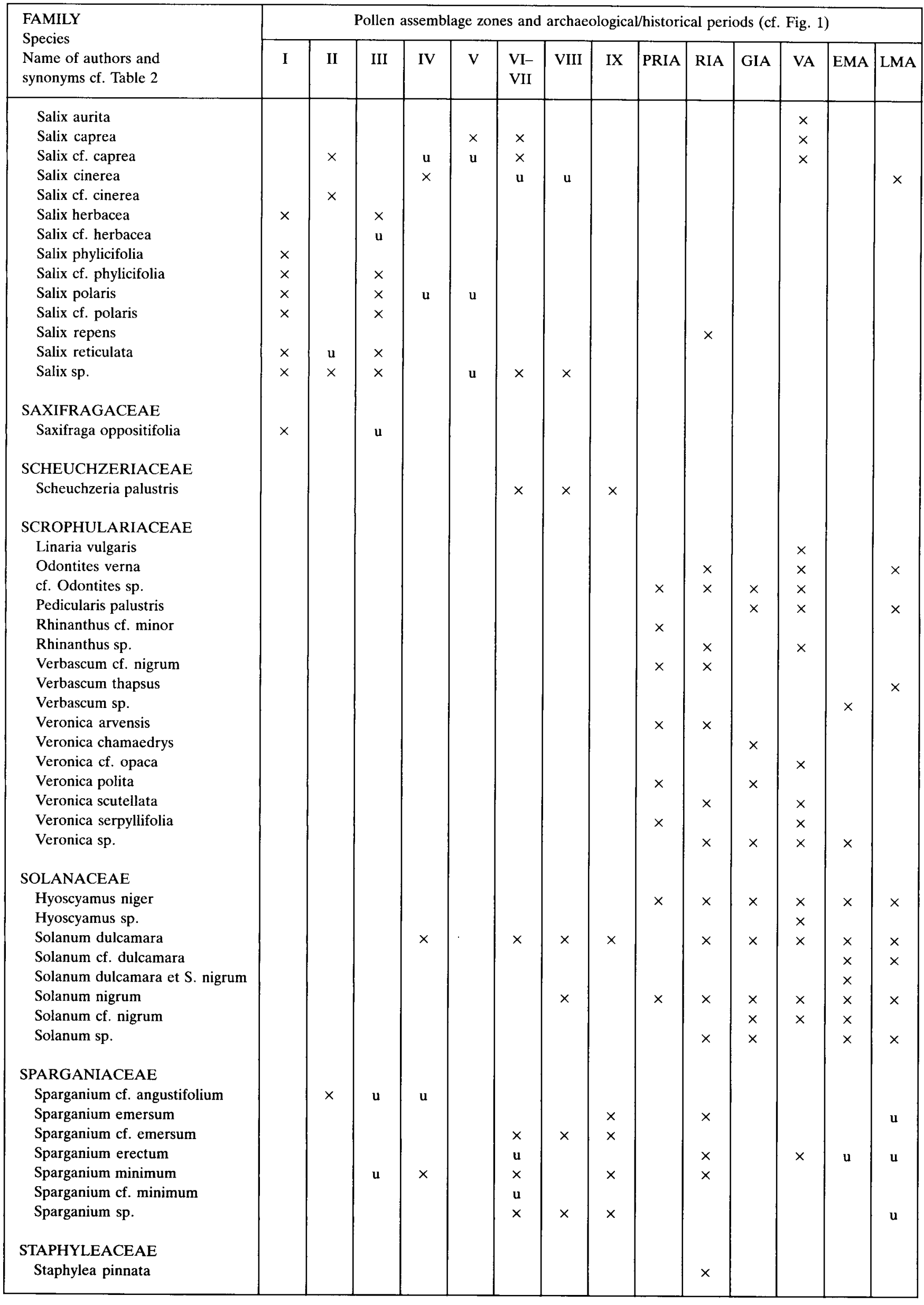




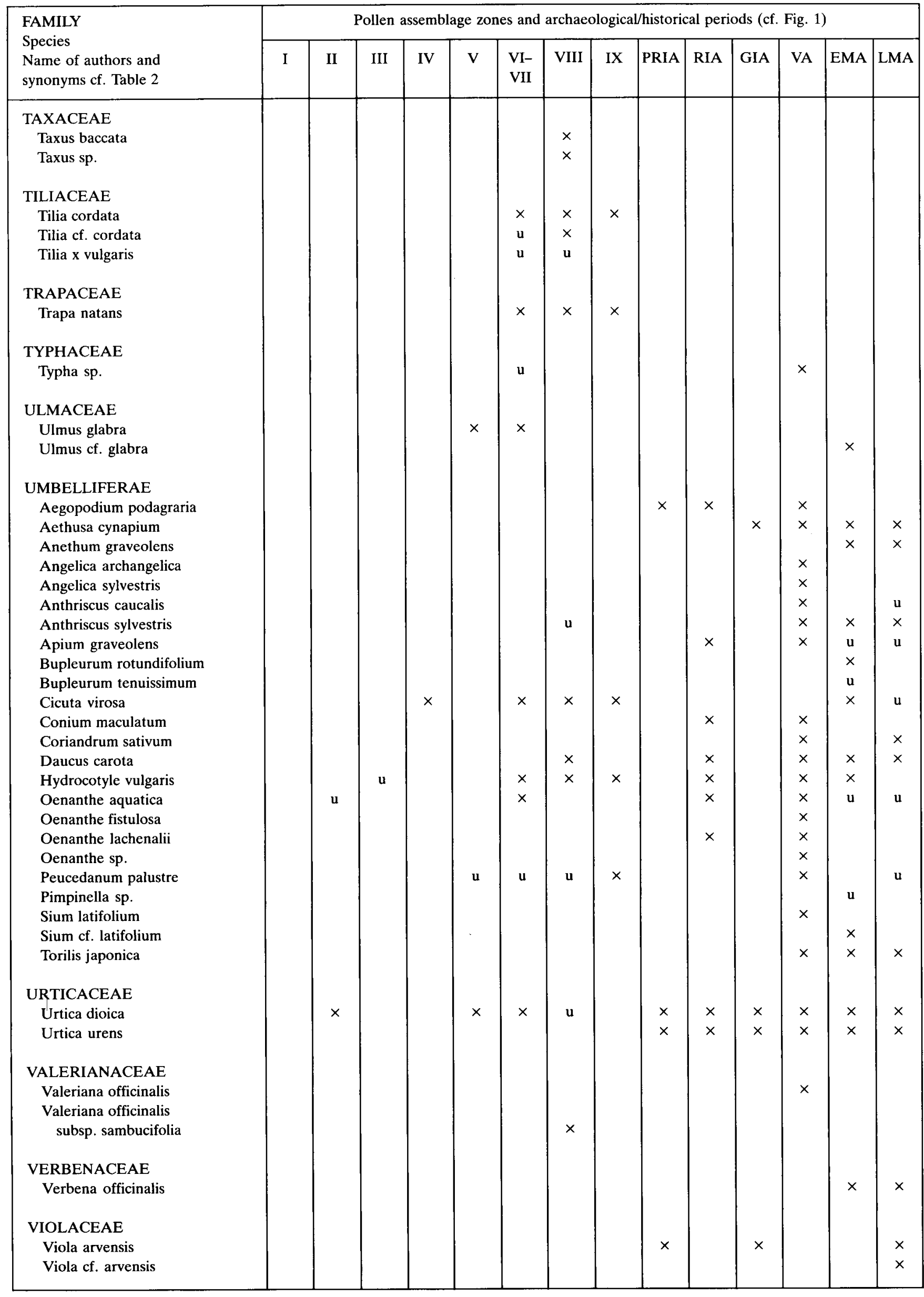




\begin{tabular}{|c|c|c|c|c|c|c|c|c|c|c|c|c|c|c|}
\hline \multirow{2}{*}{$\begin{array}{l}\text { FAMILY } \\
\text { Species } \\
\text { Name of authors and } \\
\text { synonyms cf. Table } 2\end{array}$} & \multicolumn{14}{|c|}{ Pollen assemblage zones and archaeological/historical periods (cf. Fig. 1) } \\
\hline & I & II & III & IV & V & $\begin{array}{l}\text { VI- } \\
\text { VII }\end{array}$ & VIII & IX & PRIA & RIA & GIA & VA & EMA & LMA \\
\hline $\begin{array}{l}\text { Viola arvensis, V. canina } \\
\text { et V. tricolor } \\
\text { Viola canina } \\
\text { Viola cf. canina } \\
\text { Viola palustris } \\
\text { Viola cf. palustris } \\
\text { Viola reichenbachiana } \\
\text { Viola tricolor } \\
\text { Viola sp. } \\
\text { VITACEAE } \\
\text { Vitis vinifera } \\
\text { ZANNICHELLIACEAE } \\
\text { Zannichellia palustris } \\
\text { ZOSTERACEAE } \\
\text { Zostera marina } \\
\text { Zostera noltii }\end{array}$ & & & $x$ & & $x$ & $\mathbf{u}$ & $\begin{array}{l}x \\
x\end{array}$ & $x$ & & $\begin{array}{l}x \\
x\end{array}$ & $\times$ & $\begin{array}{l} \\
\times \\
\times \\
\times \\
\times\end{array}$ & $x$ & $x$ \\
\hline
\end{tabular}


This publication summarizes published finds of macrofossils from 551 taxa of Spermatophyta originating from $\mathbf{5 0 5}$ sites in Denmark, Schleswig, Scania, Halland, and Blekinge and dated to periods between 13,000 B.P. and A.D. 1536. The information is arranged in one site map and three tables. Table 1 gives information about the site, the findings, age, dating method, and media examined. Table 2 lists the finds according to the species and age of material. Table 3 summarizes by family the finds of macrofossils in these periods. 